\section{$Y-12$}

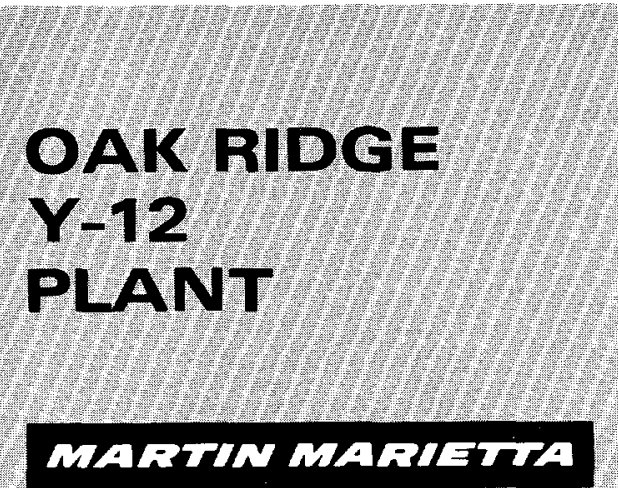

macith muarezala

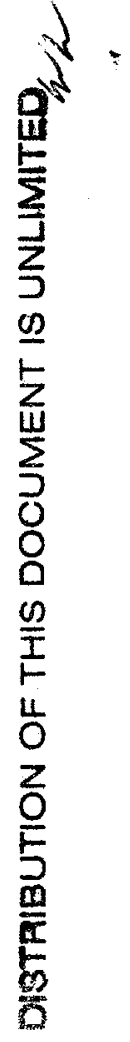

DSTPIRUT
Final Report from VFL Technologies for the Pilot-Scale Thermal Treatment of Lower East Fork Poplar Creek Floodplain Soils

\section{LEFPC Appendices \\ Volume 1 \\ Appendix I-IV}

Prepared by VFL Technology Corporation for

Martin Marietta Energy Systems

P.O. Box 2002

Oak Ridge, TN $37831-6501$

Subcontract No. 82Y-05556V

September 1994

MARTIN MARIETTA ENERGY SYSTEMS, INC.

managing the

Oak Ridge, Y-12 Plant

Oak Ridge, Tennessee

under contract DE-AC05-84OR21400 for the

U.S. Department of Energy
MANAGED BY

MARTIN MARIETTA ENERGY SYSTEMS, INC.

FOR THE UNITED STATES

DEPARTMENT OF ENERGY

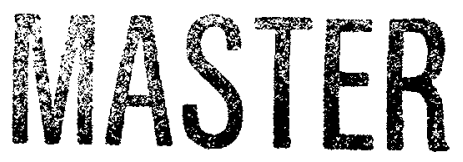

WLAMTES 


\section{DISCLAIMER}

This report was prepared as an account of work sponsored by an agency of the United States Government. Neither the United States Government nor any agency thereof, nor any of their employees, make any warranty, express or implied, or assumes any legal liability or responsibility for the accuracy, completeness, or usefulness of any information, apparatus, product, or process disclosed, or represents that its use would not infringe privately owned rights. Reference herein to any specific commercial product, process, or service by trade name, trademark, manufacturer, or otherwise does not necessarily constitute or imply its endorsement, recommendation, or favoring by the United States Government or any agency thereof. The views and opinions of authors expressed herein do not necessarily state or reflect those of the United States Government or any agency thereof. 


\section{DISCLAIMER}

Portions of this document may be illegible in electronic image products. Images are produced from the best available original document. 


\section{APPENDIX I}

\section{CALIBRATION RECORDS}


CALIBRATION OF THERMOCOUPLES Date $3-24-95$
Accuracy \pm 40 Form C-5
Du $0.75 \%$ of reading (which ever is
greater)

CALIBRATION OF THERMOCOUPLES Date $3-24-95$
Accuracy \pm 40 Form C-5
Du $0.75 \%$ of reading (which ever is
greater)

Procedure No.: NFS-EC-20, Rev. 4

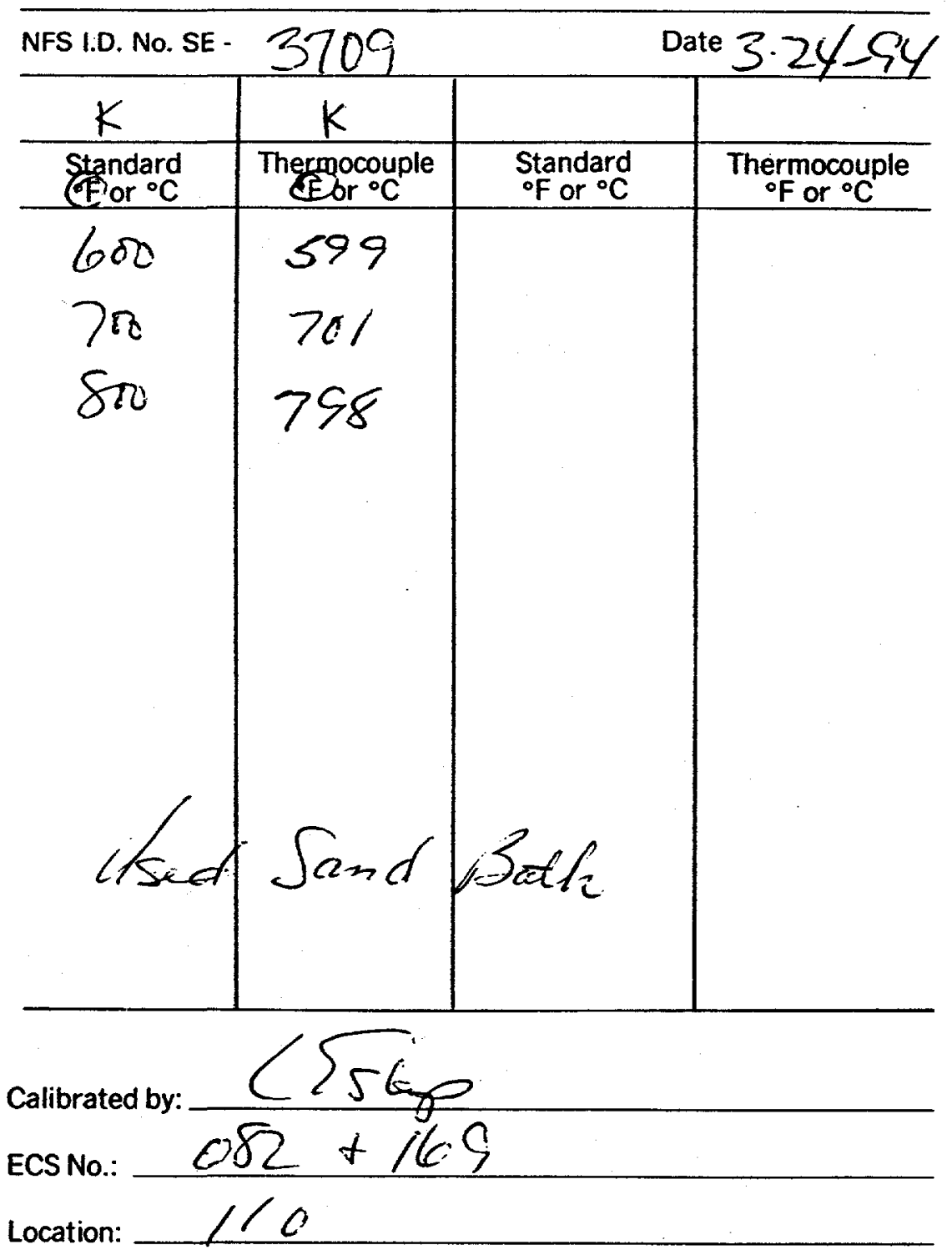

Knowingly or willfully falsifying or concealing a material fact on this form, or making false, fictitious or fraudulent statements or representations herein could constitute a felony punishable under Federal Statutes. 
Due Date $3-24-5$ Accuracy \pm joF or $\pm 0.75 \%$ of reading (which ever is
Procedure No.: NFS-EC-20, Rev. 4
greater)

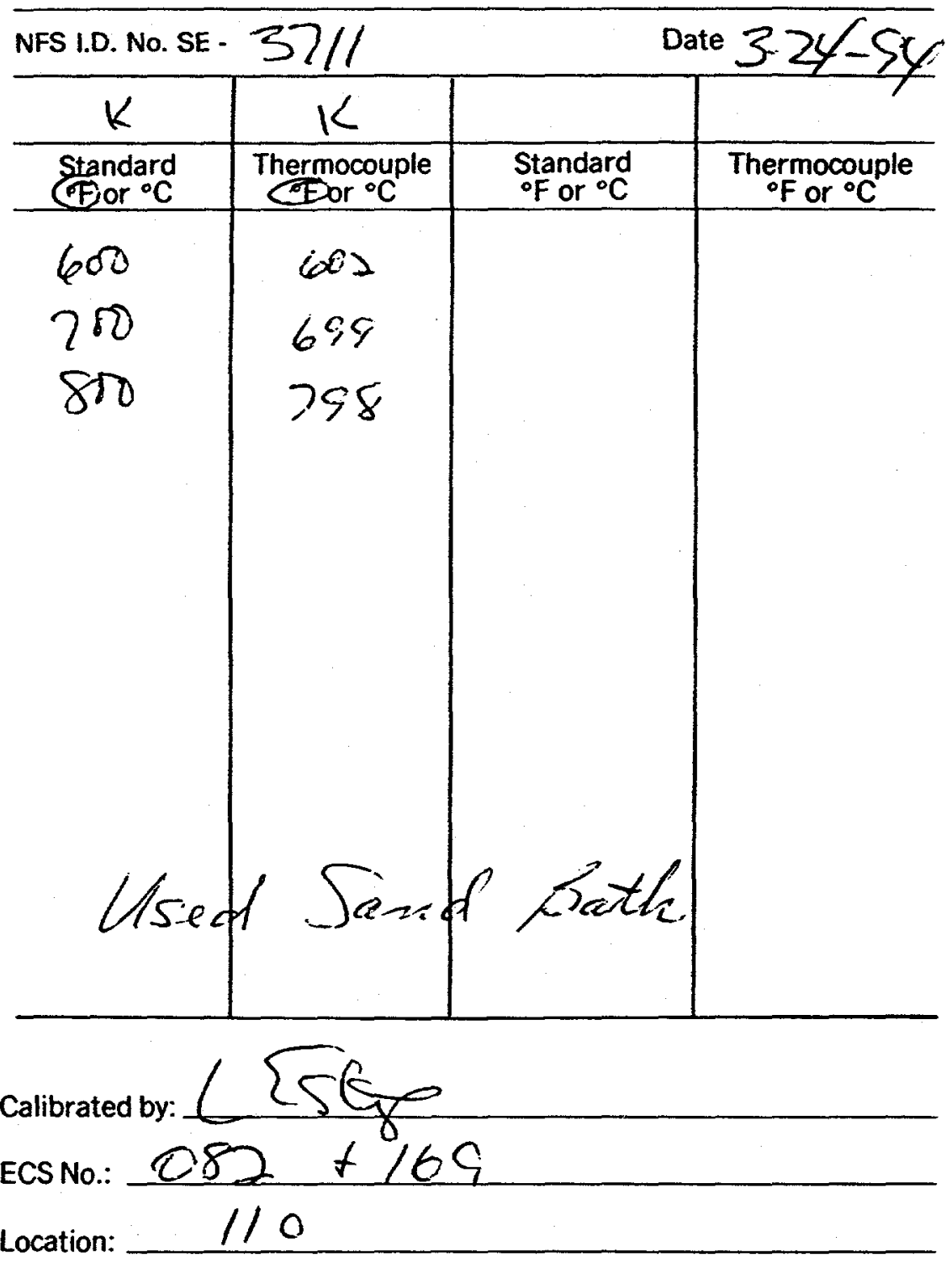

Knowingly or willfully faisifying or concealing a material fact on this form, or making false, fictitious or fraudulent statements or representations herein could constitute a felony punishable under Federal Statutes. 
CALIBRATION OF THERMOCOUPLES

Form C.5

Due Date $3 \cdot 24-95$

Accuracy \pm 4 of or $\pm 0.75 \%$ of reading (Which ever is Procedure No.: NFS-EC-20, Rev. 4 greater)

NFS I.D. No. SE - 37/2 Date $3.24-54$

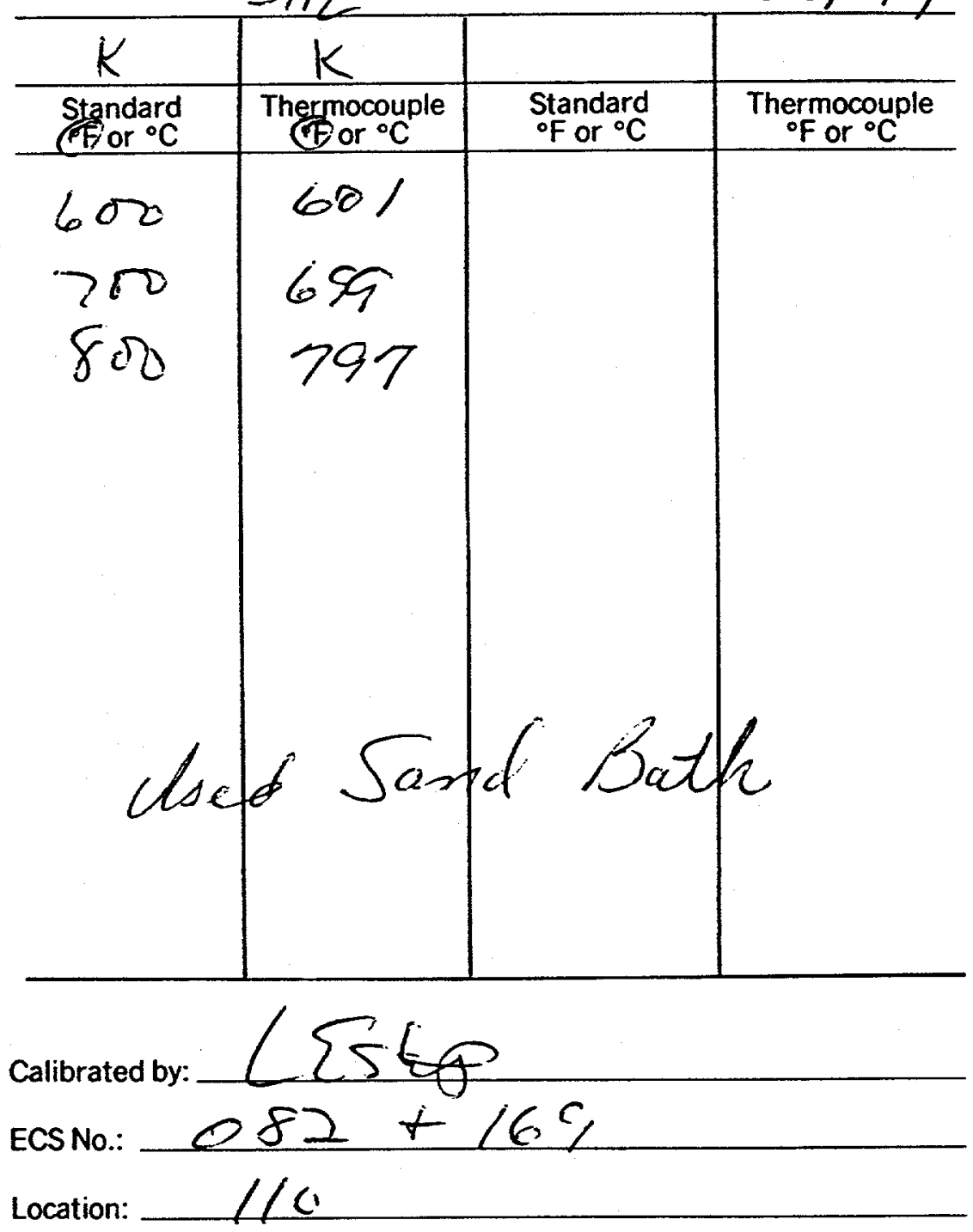

Knowingly or willfully falsifying or concealing a material fact on this form, or making false, fictitious or fraudulent statements or representations herein could constitute a felony punishable under Federal Statutes. 


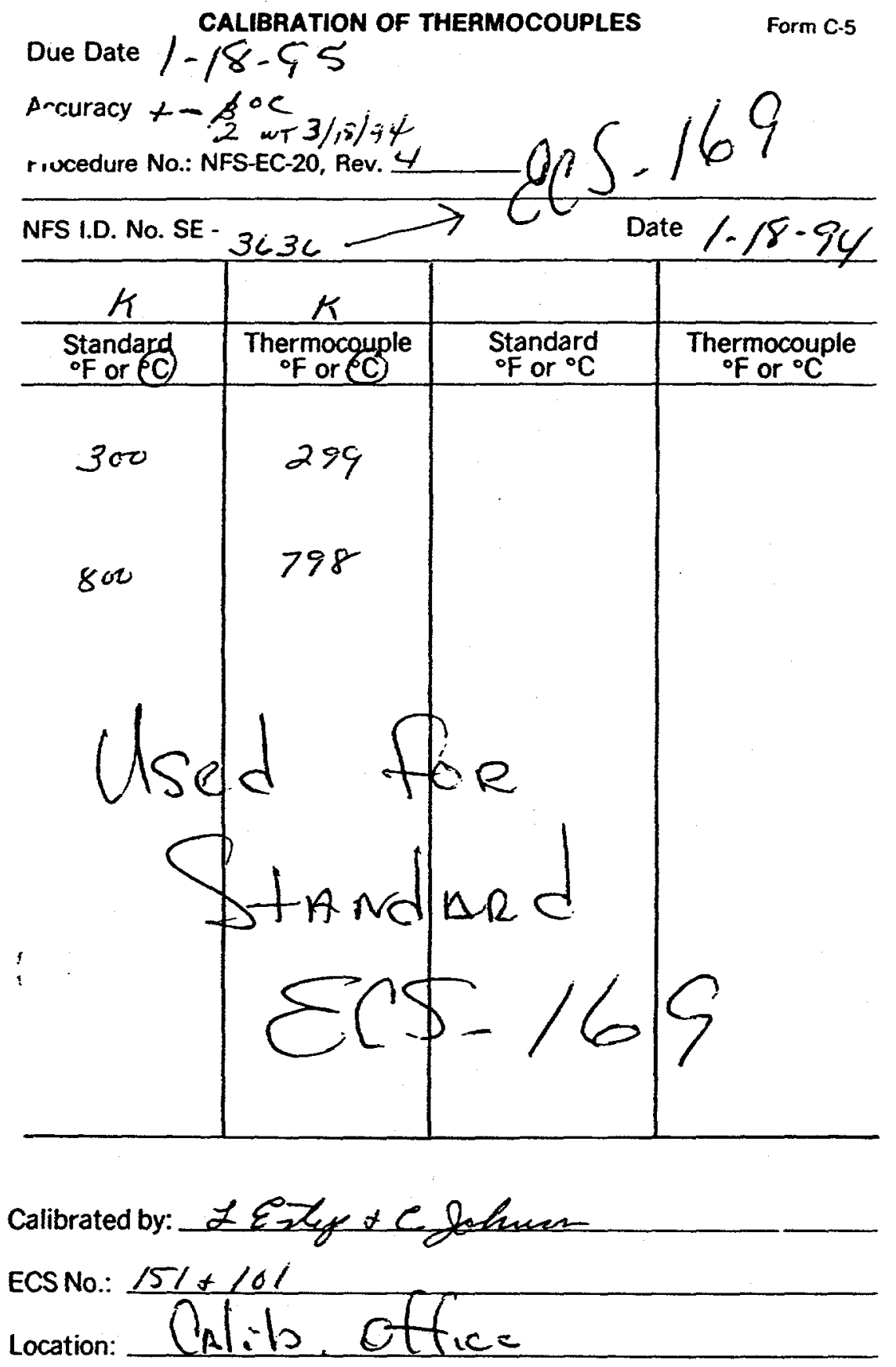

Knowingly or willfully falsifying or concealing a material fact on this form, or making false, fictitious or fraudulent statements or representations herein could constitute a felony punishable under Federal Statutes. 


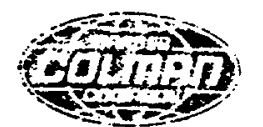

a Sisbo company

Customer:

\section{NUCLEAR FUEL SERVICES} 1205 BANNER HILL DRIVE ERIIN, TN 37650

\section{Certifícation 2 Repart}

Page 1 of 1

Customer P91-2280
Order No

B-C Order No. 985-35-074857-65

Part No.

M225-10008-000-1-06

Type /Gauge K-19

The following table compares the results of a thermocouple checked against siandards that are traceable to the National Bureau of Standaris Test No. 245792 . Temperature values in this report are based on the International Practical Temperature Scale of 1968, IPTS-68. The certification was made by Quality Conitrol personnel in accordance with Barber Colman Industrial Instruments Division procedures.

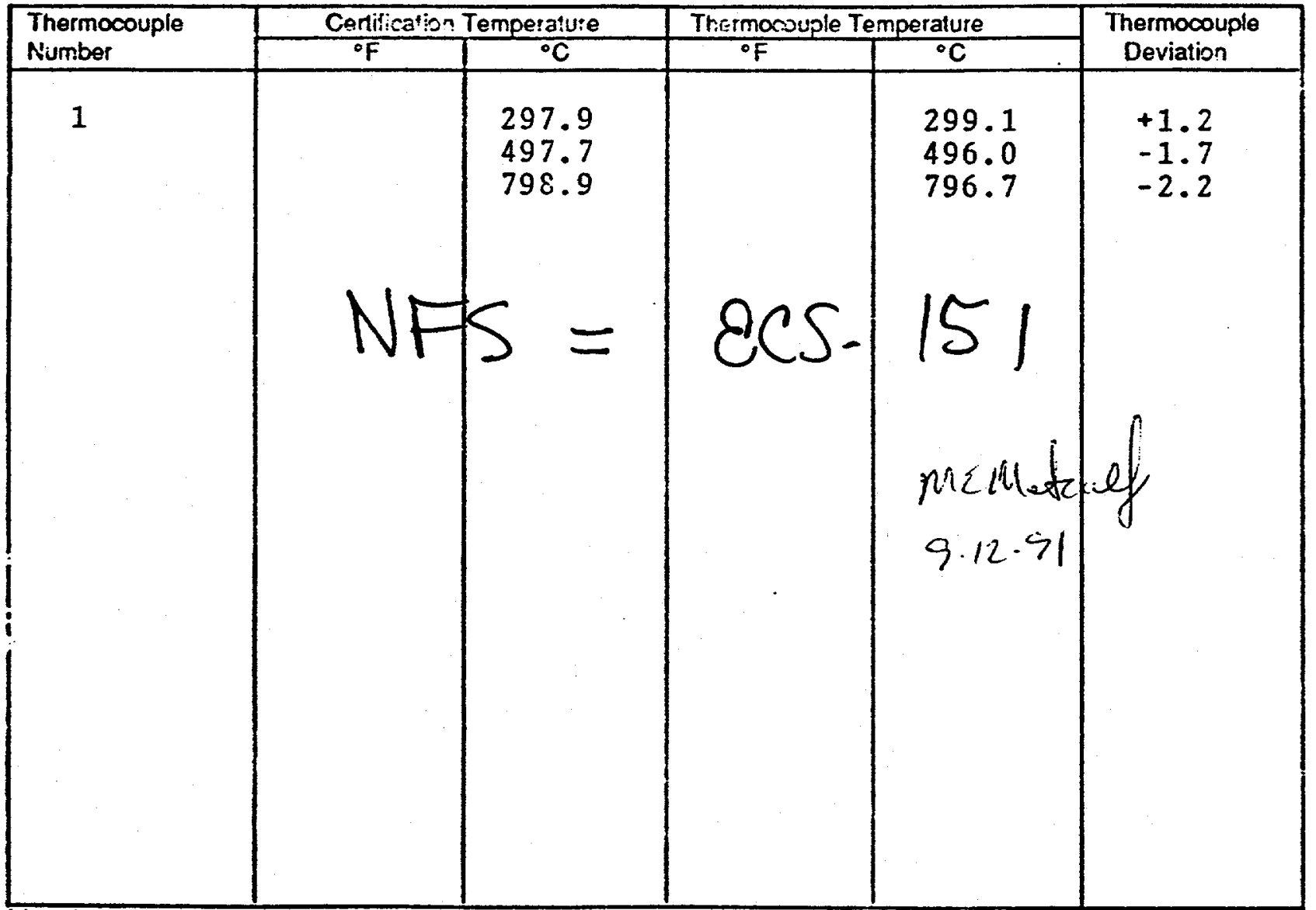

Thereby certify the items listed above were manufactured and tested in contormance vith accepted practice. It is further certified that, to the best of my knowledge, the data is true and corrert. The calibration of a thermocouple is subject to change during use. The magnitude of change depends on lactcrs such as iemperature, time and conditions of use.

\section{Earber-Colman Company} INDUSTRIAL INSTRUMENTS DIVISION 135: Citford Avenue

P. O. E.r. 2@४?

Loves Fark. IL., U.S.A 61132-2940

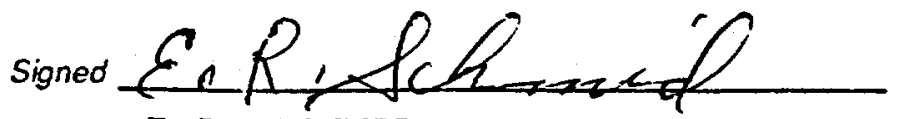

Name E.R. SCHMID

Date MARCit 5, 1991

\%

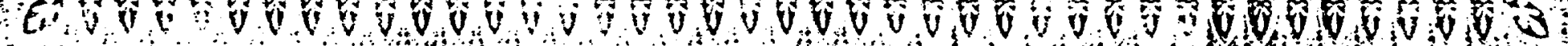




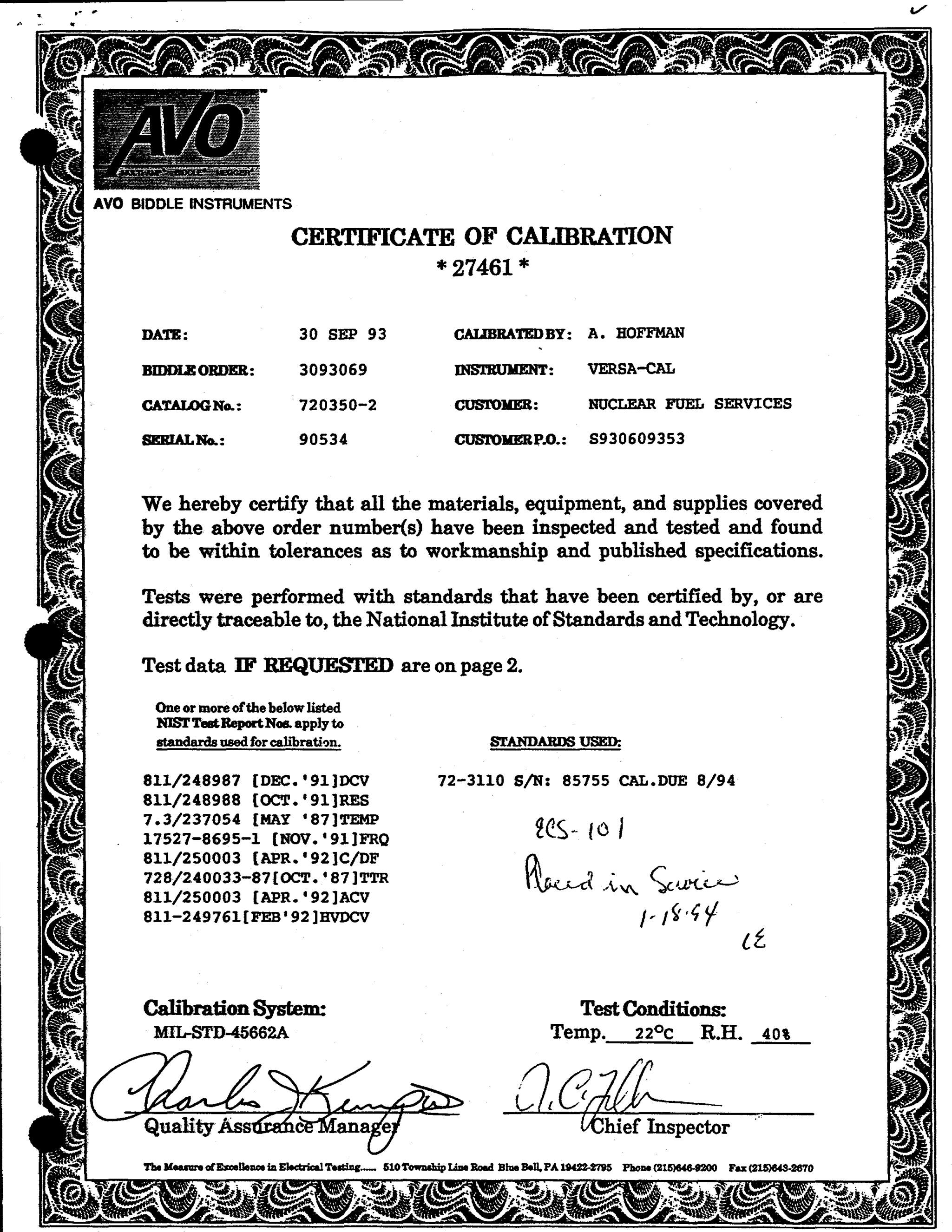




\section{RANGES FOR VERSA-CALT INSTRUMENTS USING 1968 IPTS TABLES:}

\begin{tabular}{|c|c|c|c|c|c|c|}
\hline \multirow{2}{*}{$\begin{array}{l}\text { Catalog } \\
\text { Number }\end{array}$} & \multirow{2}{*}{$\begin{array}{l}\text { TiC Typo } \\
\text { Selected on } \\
\text { Range Switch }\end{array}$} & \multicolumn{2}{|c|}{$\begin{array}{l}\text { Ringe. } \\
\text { Mousure and Output }\end{array}$} & \multirow{2}{*}{$\begin{array}{l}\text { Pesolution. } \\
\text { Pepeatability. } \\
\text { Moasure and Outpen }\end{array}$} & \multicolumn{2}{|c|}{ 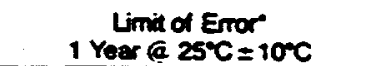 } \\
\hline & & $F$ & $c$ & & $F$ & ${ }^{\circ} \mathrm{C}$ \\
\hline \multirow{4}{*}{$720350-1,3$} & \rfloor & $-34680+2192$ & $-21000+1200$ & $0.1^{\circ}$ & \pm 1 & \pm 0.6 \\
\hline & $\mathbf{K}$ & -328 to +2501 & $-20000+1372$ & $0.1^{\circ}$ & \pm 1 & $=0.6$ \\
\hline & $\mathbf{T}$ & $\begin{array}{l}-337 \text { to }+752 \\
-405 \text { to }-337\end{array}$ & $\begin{array}{l}-205 t 0+400 \\
-243 t 0-205\end{array}$ & $\begin{array}{l}0.1^{\circ} \\
0.1^{\circ}\end{array}$ & $\begin{array}{l}=1 \\
=10\end{array}$ & $\begin{array}{l} \pm 0.6 \\
\pm 5.6\end{array}$ \\
\hline & mv & \multicolumn{2}{|c|}{$\begin{array}{c}-1010-20 \mathrm{mV} \\
-2010+20 \mathrm{mV} \\
+2010+101.1 \mathrm{mv} \\
\text { (Output: }-2010+101.1 \mathrm{mV} \text { unioaded }\end{array}$} & $\begin{array}{l}0.01 \mathrm{mv} \\
0.001 \mathrm{mv} \\
0.01 \mathrm{mv}\end{array}$ & \multicolumn{2}{|c|}{$\begin{array}{l}=(0.03 \% \text { of reading }+2 \text { digits }) \\
=(0.03 \% \text { of reading }+5 \text { digits }) \\
=(0.03 \% \text { of reading }+2 \text { digits })\end{array}$} \\
\hline \multirow{5}{*}{$720350-2,4$} & \multicolumn{6}{|c|}{ TIC TYPes \&, K, T, mV some as for Cat No. T20350-1 Listed Above, plus . . . } \\
\hline & $\mathbf{E}$ & $\begin{array}{l}-38910+1832 \\
-422 \text { to }-389\end{array}$ & $\begin{array}{l}-23410-1000 \\
-25260-234\end{array}$ & $\begin{array}{l}0.1^{\circ} \\
0.1^{\circ}\end{array}$ & $\begin{array}{l} \pm 1 \\
\pm 5\end{array}$ & $\begin{array}{l}=0.6 \\
\pm 2.8\end{array}$ \\
\hline & $\mathbf{A}$ & $-5810+3214$ & $-5000+1768$ & $1.0^{\circ}$ & \pm 2 & $=1.1$ \\
\hline & $\mathbf{S}$ & $-5810+3214$ & $-500+1768$ & $1.0^{\circ}$ & \pm 2 & \pm 1.1 \\
\hline & C & $\begin{array}{l}+3210+2192 \\
219210+3812 \\
381210+4200\end{array}$ & $\begin{array}{r}010-1200 \\
120010-2100 \\
2100 \text { to }+2315\end{array}$ & $\begin{array}{l}1.0^{\circ} \\
1.0^{\circ} \\
1.0^{\circ}\end{array}$ & $\begin{array}{l} \pm 1 \\
\pm 2 \\
\pm 3\end{array}$ & $\begin{array}{l} \pm 0.6 \\
\pm 1.1 \\
\pm 1.7\end{array}$ \\
\hline
\end{tabular}

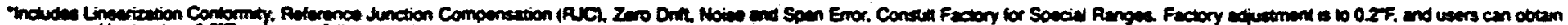

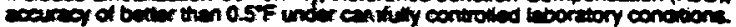

Thermocouples:

1 hon-Constantan

$K$ Chre mot Alume

T Copper-Constanten

E Crroma-Constanten

C Tungster. $5 \%$ PtreniumTungseren 26\% Phenim
A Platinum. 13\% Phodium Platinum

$S$ Platinum. $10 \%$

Ahodium Platinum
N Nicrosit Nisil

NM Nickot-Nickel, 18\% Mohy O (W3) Tungsten, 3\% ATemium Tungsten 25\% Rhenium

G (IV) Tungster Tungsten. 26\% Phonium

B Platinum $30 \%$ Phenium-

Platinum, 6\% Phenium

PL2 Platined if (Engethard)

\section{SPECIFICATIONS FOR LOOP MODULE}

Rarges, Resolution and Accurecy - MEASURE Chmmel

\begin{tabular}{|c|c|c|c|}
\hline $\begin{array}{l}\text { Units of } \\
\text { Measure }\end{array}$ & $\begin{array}{c}\text { Range ( + or -) } \\
\text { (Auto Change at 19.95) }\end{array}$ & Resolution & $\begin{array}{l}\text { Limit of Error } \\
25=10^{\circ} \mathrm{C} \text {. One Year }\end{array}$ \\
\hline \multirow{2}{*}{ ma } & $0.000-19.950$ & 0.001 & $0.05 \% \mathrm{rdg}+0.005$ \\
\hline & 19.95-101.10 & 0.01 & $0.05 \% \mathrm{rog}+0.02$ \\
\hline \multirow{2}{*}{ Wots } & $0.000-19.950$ & 0.001 & $0.05 \%$ dig +0.005 \\
\hline & $19.95-60.0$ & 0.01 & $0.05 \% \mathrm{rdg}+0.02$ \\
\hline
\end{tabular}

Ranger, Resolution and Accuracy-OUTPUT Chame

\begin{tabular}{|c|c|c|c|c|}
\hline \multirow{2}{*}{$\begin{array}{l}\text { Nomina } \\
\text { Control Loop } \\
\text { Range Setting }\end{array}$} & \multirow[b]{2}{*}{$\begin{array}{l}\text { Full Output } \\
\text { Range }\end{array}$} & \multirow{2}{*}{$\begin{array}{l}\text { Output Setting* } \\
\text { Limit of Error at Fixed Points } \\
\text { (Fine trim at dot) }\end{array}$} & \multicolumn{2}{|c|}{ Oisplay (Auto Change at 19.95) } \\
\hline & & & $\begin{array}{l}\text { Resolution } \\
\text { (mA or Votts) }\end{array}$ & $\begin{array}{l}\text { Limit of Error } \\
25^{\circ}=10^{\circ} \text {. One Year }\end{array}$ \\
\hline $\begin{array}{r}4-20 \mathrm{~mA} \\
10-50 \mathrm{~mA}\end{array}$ & $\begin{array}{l}0-22 \mathrm{~mA} \\
0.55 \mathrm{~mA}\end{array}$ & $\begin{array}{l}0.024 \mathrm{~mA}(0.15 \% \text { range) } \\
0.10 \mathrm{~mA}(0.25 \% \text { range })\end{array}$ & \multirow{2}{*}{\multicolumn{2}{|c|}{$\begin{array}{l}\text { Same as in } \\
\text {-MEASURE Chamel "Table }\end{array}$}} \\
\hline $\begin{array}{l}1-5 \mathrm{~V} \\
2.10 \mathrm{~V}\end{array}$ & $\begin{array}{l}0-5.5 \mathrm{~V} \\
0.11 \mathrm{~V}\end{array}$ & $\begin{array}{l}0.01 V(0.25 \% \text { range) } \\
0.02 V(0.25 \% \text { range) }\end{array}$ & & \\
\hline
\end{tabular}

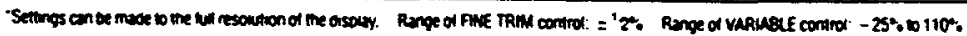


AVO INTERNATIONAL

SHEET_3_of 3

CALIERATION DATA SHEET

DATE: SEFT. 30,1993

AS RECEIUED:

BERIAL NO. 98534

AS BHIPPED:

CAT. NO.: 72035Q-2

CAL. BY: A. HOFFMAN

BIDDLE ORDER: 3093069

CUBTOMER ORDER: S930689353

CUSTOMER: NUCLEAR FUEL SERUICES

- - - SPECIAL REAdings - - -

LIMIT OF ERROR: +/- 10 F NOTEः FOR STANDARDS USED, SEE SHEET \# 1

\begin{tabular}{|c|c|c|c|}
\hline $\begin{array}{l}\text { THERMOCOUPLE } \\
\text { TYPE }\end{array}$ & $\begin{array}{l}\text { IV POT. } \\
\text { BETTINB }\end{array}$ & $\begin{array}{l}\text { NOMINAL READING } \\
\text { IN } \bullet F\end{array}$ & $\begin{array}{l}\text { ACTUAL RDG. } \\
\text { IN } \cdot F\end{array}$ \\
\hline $\mathrm{J}$ & -7.519 & -300 & -300.0 \\
\hline $\mathrm{J}$ & -4.678 & -150 & -150.0 \\
\hline$J$ & 5. 268 & E1E & อ12.ด \\
\hline $\mathrm{J}$ & 29.515 & $108 a$ & 1ana. a \\
\hline$K$ & 2こ். 251 & 1 Rera & 1000.1 \\
\hline$K$ & 54.845 & esqu & esena. 1 \\
\hline$T$ & - 2. 581 & -100 & -100.1 \\
\hline$T$ & 13.834 & 540 & 539.9 \\
\hline
\end{tabular}

QA FORM CALSHEET 4/9E V-CAL-1 
RANGES FOR VERSA-CALT INSTRUMENTS USING 1968 IPTS TABLES:

\begin{tabular}{|c|c|c|c|c|c|c|}
\hline \multirow{2}{*}{ Catiog } & \multirow{2}{*}{$\begin{array}{l}\text { TrC Type } \\
\text { Selocted on } \\
\text { Alenge Switch }\end{array}$} & \multicolumn{2}{|c|}{$\begin{array}{l}\text { Penge, } \\
\text { Mnesire and outpert }\end{array}$} & \multirow{2}{*}{$\begin{array}{l}\text { Pesolurion. } \\
\text { Propeatability. } \\
\text { Moesure and Outpert }\end{array}$} & \multicolumn{2}{|c|}{$\begin{array}{l}\text { Limi of Error } \\
1 \text { Yoer } @ 25^{\circ} \mathrm{C}=10^{\circ} \mathrm{C}\end{array}$} \\
\hline & & $F$ & $c$ & & $F$ & ${ }^{2} \mathrm{C}$ \\
\hline \multirow{4}{*}{$720350-1,3$} & $\mathbf{J}$ & $-34610+2192$ & $-21000+1200$ & $0.1^{\circ}$ & \pm 1 & \pm 0.6 \\
\hline & $\mathbf{K}$ & -328 to +2501 & $-20000+1372$ & $0.1^{\circ}$ & \pm 1 & $=0.6$ \\
\hline & $\mathbf{T}$ & $\begin{array}{l}-33710+752 \\
-40510-337\end{array}$ & $\begin{array}{l}-2056+400 \\
-2430-205\end{array}$ & $\begin{array}{l}0.1^{\circ} \\
0.1^{\circ}\end{array}$ & $\begin{array}{l} \pm 1 \\
\pm 10\end{array}$ & $\begin{array}{l} \pm 0.6 \\
\pm 5.6\end{array}$ \\
\hline & $\mathbf{m v}$ & $\begin{array}{r}-101 \\
-20 \\
+20 \\
\text { 1Output: } 2010 \\
\end{array}$ & $\begin{array}{l}20 m \mathrm{~V} \\
20 \mathrm{~V} V \\
101.1 \mathrm{mV} \\
1.1 \mathrm{mV} \text { unloadad }\end{array}$ & $\begin{array}{l}0.01 \mathrm{mV} \\
0.001 \mathrm{mV} \\
0.01 \mathrm{mV}\end{array}$ & $\begin{array}{l}=10.03 \% \\
=0.03 \% \\
=(0.03 \%\end{array}$ & $\begin{array}{l}+2 \text { digits) } \\
+5 \text { digits } \\
+2 \text { digits }\end{array}$ \\
\hline \multirow{5}{*}{ 720350-2.4 } & \multicolumn{6}{|c|}{ Tic TrPes d, K, T, mV seme as for Cat. No. 720350-1 Leted Above, plus. . . } \\
\hline & $\mathbf{E}$ & $\begin{array}{l}-38910+1832 \\
-42210-389\end{array}$ & $\begin{array}{l}-23410-1000 \\
-25200-234\end{array}$ & $0.1^{\circ}$ & $\begin{array}{l} \pm 1 \\
\pm 5\end{array}$ & $\begin{array}{l}=0.6 \\
=2.8\end{array}$ \\
\hline & $\mathbf{R}$ & $-5810+3214$ & -50 to +1768 & $1.0^{\circ}$ & \pm 2 & \pm 1.1 \\
\hline & $\mathbf{s}$ & $-58 t 0+3214$ & $-50 t 0+1768$ & 1.0 & \pm 2 & \pm 1.1 \\
\hline & C & $\begin{array}{l}+3210+2192 \\
219280+3812 \\
381280+4200\end{array}$ & $\begin{array}{r}0 \text { to }-1200 \\
1200 \text { to }-2100 \\
2100 \text { to }+2315\end{array}$ & $\begin{array}{l}1.0^{\circ} \\
1.0^{\circ} \\
1.0^{\circ}\end{array}$ & $\begin{array}{l} \pm 1 \\
\pm 2 \\
\pm 3\end{array}$ & $\begin{array}{l}=0.6 \\
\pm 1.1 \\
\pm 1.7\end{array}$ \\
\hline
\end{tabular}

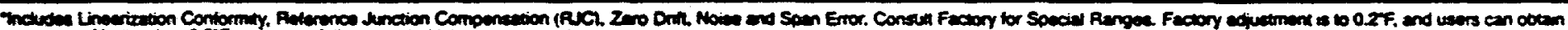

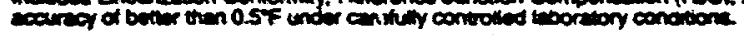

Treminocouplea: IJ ron-Constantan K Copper-Constanten
E Crrome-Constantan C Tungren. 5\% RhenimTungsion 26\% Rherium
A Platinum. 13\% Rhodium Platinum

5 Platimum. $10 \%$ Phodium Platinum
N Nicrosit Nisid

NM Nicket-Nicket, $18 \%$ Moly 0 (w3) Tungsten, $3 \%$ Phienium Tungsten, $25 \%$ Rherium

$G$ (M) Tungsten Tungsien. $26 \%$ Phonium

8 Piatinum, $30 \%$ Rhemium

Piatinum $6 \%$ Rhenium

PL2 Platina if (Engethard)

\section{SPECIFICATIÓNS FOR LOOP MODULE}

Ranjea, Resohrtion and Aceurecy-MEASURE Chamed

\begin{tabular}{|c|c|c|c|}
\hline $\begin{array}{c}\text { Units of } \\
\text { Measure }\end{array}$ & $\begin{array}{c}\text { Range }(+o f-1 \\
\text { (Auto Change at 19.95) }\end{array}$ & Resolution & $\begin{array}{c}\text { Linit of Error } \\
25=100 \text {. One Year }\end{array}$ \\
\hline \multirow{2}{*}{$\mathrm{ma}$} & $0.000-19.950$ & 0.001 & $0.05 \% \mathrm{rdg}+0.005$ \\
\cline { 2 - 5 } & $19.95-101.10$ & 0.01 & $0.05 \% \mathrm{rdg}+0.02$ \\
\hline \multirow{2}{*}{ vouts } & $0.000-19.950$ & 0.001 & $0.05 \% \mathrm{rdg}+0.005$ \\
\cline { 2 - 5 } & $19.95-60.0$ & 0.01 & $0.05 \% \mathrm{rog}+0.02$ \\
\hline
\end{tabular}

Ranges, Resolution and Accuracy-OUTPUT Channel

\begin{tabular}{|c|c|c|c|c|}
\hline \multirow{2}{*}{$\begin{array}{l}\text { Nominal } \\
\text { Control L000 } \\
\text { Range Seting }\end{array}$} & \multirow[b]{2}{*}{$\begin{array}{l}\text { Full Outrout } \\
\text { Range }\end{array}$} & \multirow{2}{*}{$\begin{array}{l}\text { Output Setting" } \\
\text { Limit of Error at Fixed Points } \\
\text { (Fine trim at dot) }\end{array}$} & \multicolumn{2}{|c|}{ Disptay (Auto Change at 19.95 ) } \\
\hline & & & $\begin{array}{l}\text { Resolution } \\
\text { (mA or Volts) }\end{array}$ & $\begin{array}{c}\text { Limit of Error } \\
25^{\circ}=10^{\circ} \text {. One Year }\end{array}$ \\
\hline $\begin{array}{r}4-20 \mathrm{~mA} \\
10-50 \mathrm{~mA}\end{array}$ & $\begin{array}{l}0.22 \mathrm{~mA} \\
0-55 \mathrm{~mA}\end{array}$ & $\begin{array}{l}0.024 \mathrm{~mA}(0.15 \% \text { range) } \\
0.10 \mathrm{~mA}(0.25 \% \text { range) }\end{array}$ & \multirow{2}{*}{\multicolumn{2}{|c|}{$\begin{array}{c}\text { Same as in } \\
\text {-MEASUAE Crannel " Table }\end{array}$}} \\
\hline $\begin{array}{l}1-5 \mathrm{~V} \\
2.10 \mathrm{~V}\end{array}$ & $\begin{array}{l}0-5.5 V \\
0-11 V\end{array}$ & $\begin{array}{l}0.01 \mathrm{~V}(0.25 \% \text { range) } \\
0.02 \mathrm{~V}(0.25 \% \text { range) }\end{array}$ & & \\
\hline
\end{tabular}

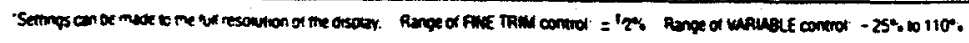


SHEET 3 of 4

CALIBRATION DATA BHEEI

DATE: JULY 6, 1992

EERIAL NO.: 82017

CAT. NO. : 720350-2

BIDDLE ORDER: 2063316

CUSTOMER: NUCLEAR FUEL SERUICES

$$
\text { - - - - gPECIAL REAdines - - - - }
$$

LIMIT OF ERROR: +1- $1^{\circ} \mathrm{F}$

\begin{tabular}{|c|c|c|c|}
\hline $\begin{array}{l}\text { THERMOCOUPLE } \\
\text { TYPE }\end{array}$ & $\begin{array}{l}\text { WV POT. } \\
\text { SETTING }\end{array}$ & $\begin{array}{l}\text { NOMINAL READING } \\
\text { IN }{ }^{\circ} \mathrm{F}\end{array}$ & $\begin{array}{l}\text { ACTUAL RDG. } \\
\text { IN } \text { OF }\end{array}$ \\
\hline $\mathrm{J}$ & -7.519 & -300 & -298.9 \\
\hline $\mathbf{J}$ & -4.678 & -150 & -149.3 \\
\hline$J$ & 5.268 & 212 & 212.5 \\
\hline $\mathbf{J}$ & 29.515 & 1000 & 1000.4 \\
\hline K & 22. 251 & 1000 & 1000.3 \\
\hline$k$ & 54.845 & 2500 & 2500.3 \\
\hline$T$ & -2.581 & -100 & -99.2 \\
\hline$T$ & 13.834 & 540 & 540.3 \\
\hline
\end{tabular}

QA FORM CALSHEET $4 / 92$

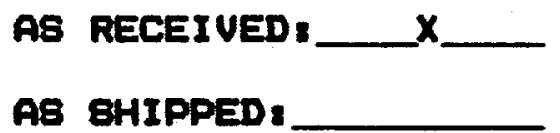

CAL. BY: M. SHOOKLA CUBTOMER ORDER: S9205 03310 
Phone: 215/646-9200

FAX. 215/643-2670

Orders: 1-800-366-5543

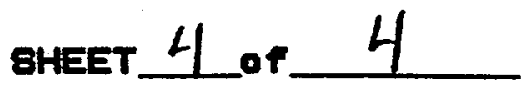

CAL IBRATION DATA BHEET

DATE: JULY 6, 1992

BERIAL NO. B BEO17

CAT. NO.: 720350-2

BIDDLE ORDERI 2063316

CUBTOMER: NUCLEAR FUEL SERUICES
AS RECEIUED

A8 EHIPPED:

CAL. BY: M. SHOOKLA CUBTOMER ORDER: S9205 03310

$$
\text { - - - EPECIAL REAdING8 - - - - }
$$

LIMIT OF ERROR: $+1-1^{\circ} \mathrm{F}$

\begin{tabular}{|c|c|c|c|}
\hline $\begin{array}{c}\text { THERMOCOUPLE } \\
\text { TYPE }\end{array}$ & $\begin{array}{c}\text { WV POT. } \\
\text { SETTING }\end{array}$ & $\begin{array}{c}\text { NOMINAL READING } \\
\text { IN }\end{array}$ & $\begin{array}{c}\text { ACTUAL RDG. } \\
\text { IN }\end{array}$ \\
\hline $\mathrm{J}$ & -7.519 & -300 & -298.8 \\
$\mathrm{~J}$ & -4.678 & -150 & -150.0 \\
$\mathrm{~J}$ & 5.268 & 212 & 211.9 \\
$\mathrm{~J}$ & 29.515 & 1000 & 999.9 \\
$\mathrm{~K}$ & 2.251 & 1000 & 999.9 \\
$\mathrm{~K}$ & 54.845 & 2500 & 2499.8 \\
$\mathrm{~T}$ & & -100 & -99.9 \\
$\mathrm{~T}$ & 13.834 & 540 & 540.0 \\
& & & \\
\hline
\end{tabular}

QA FORM CALSHEET $4 / 92$ V-CAL-1 
Reference:

ASTM Designation: E230-87, "Standard Temperature-Electromotive Force (EMF) Standardized Thermocouples" 
N.F.S. I.D. NO. $D A-580$ DESCRIPTION tResSURe Gage, MODEL NO CALIBRATION FREQUENCY (lyaundoly
MANUFACTURER $\quad \mathrm{C} S \mathrm{SC}$

Serial No. Done

CALIBRATION PROCEDURE No. \&C -5

ACCURACY $\pm 2 D S$

RANGe $0-100$ Psi

\begin{tabular}{c|l|l} 
DATE & CALIBRATION
\end{tabular}

\begin{tabular}{c|c|c} 
CALIBRATED & INITIALS & DUE DATE \\
1-19-94 & (32 & $1-19-$
\end{tabular} 
CALIBRATION OF PRESSURE GAUGES

Form $\mathrm{C}-6$

NFS I.D. No. PA - $580 \quad$ Date $\angle 19-9 \mathrm{C}$

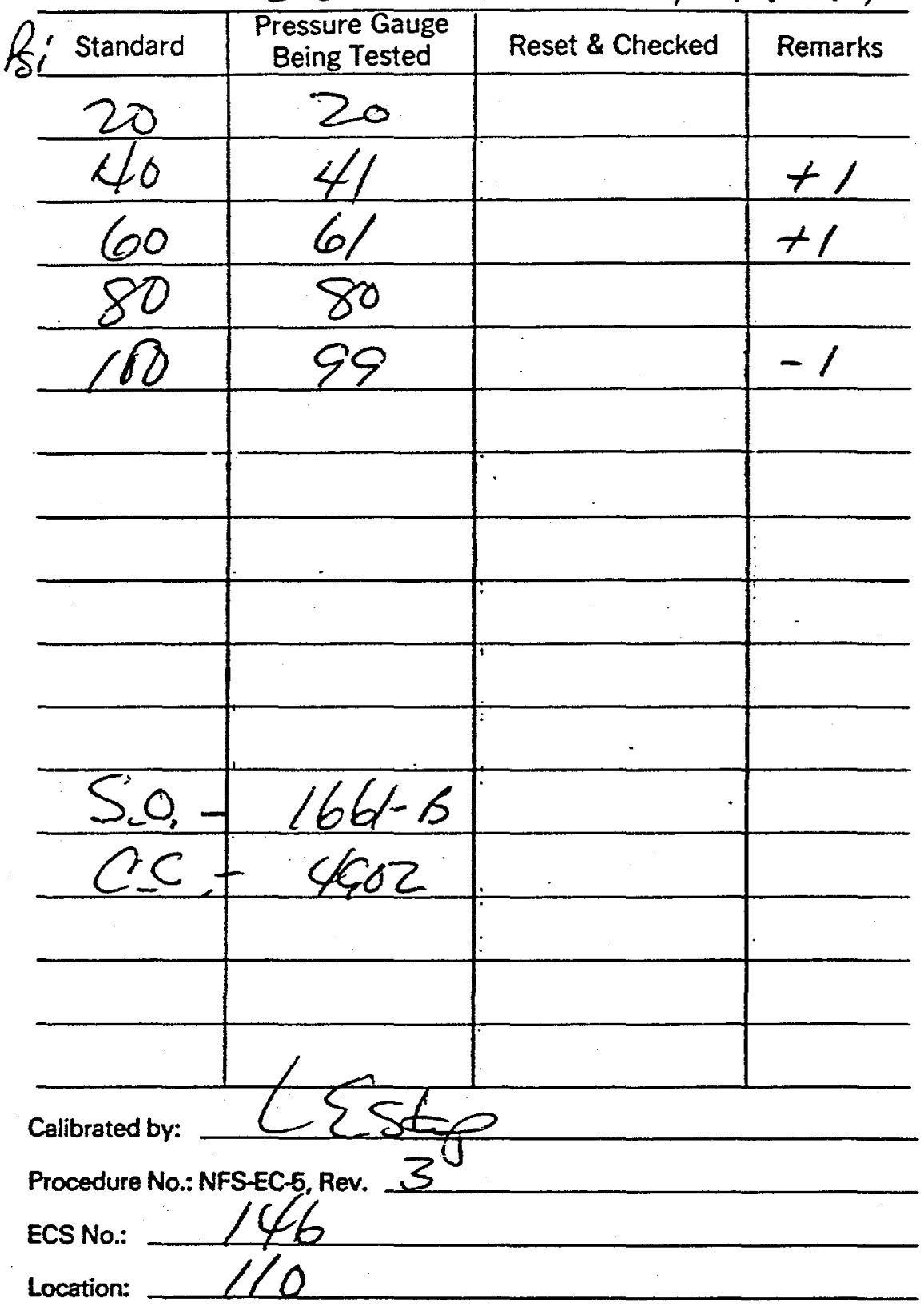

Knowingly or willfully falsifying or concealing a material fact on this form, of making false, fictitious or fraudulent statements of representations herein could constitute a felony punishable under Federal Statutes. 


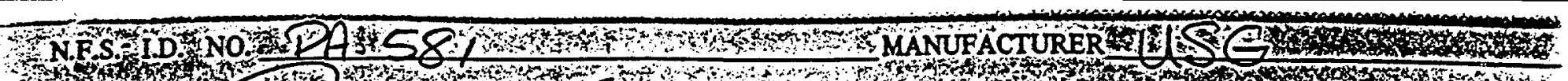

MODEL NO.

None

CALIBRATION PROCEDURE NO

CALIBRATION FREQUENCY Ommaly

ACCURACY $\pm 2 P_{S}$

RANGE $0-100$ PS i

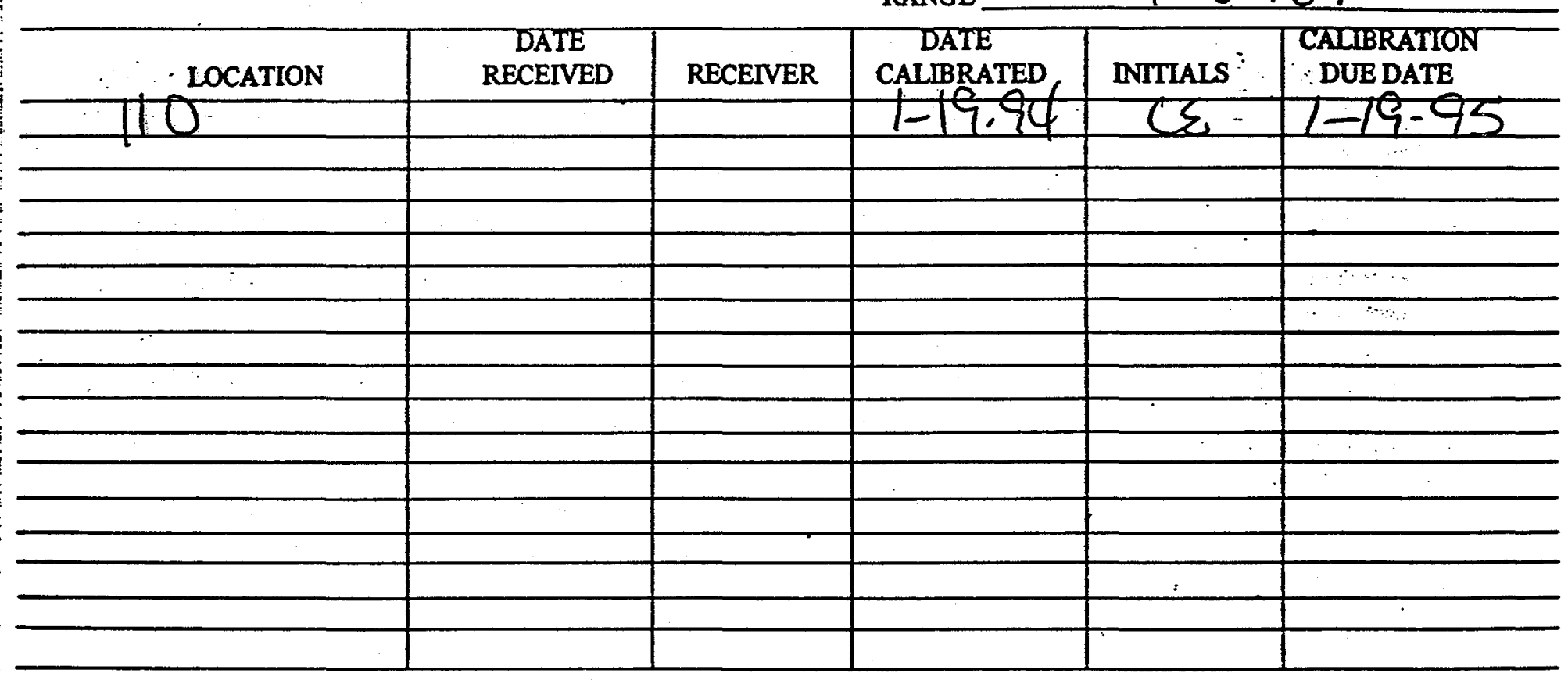




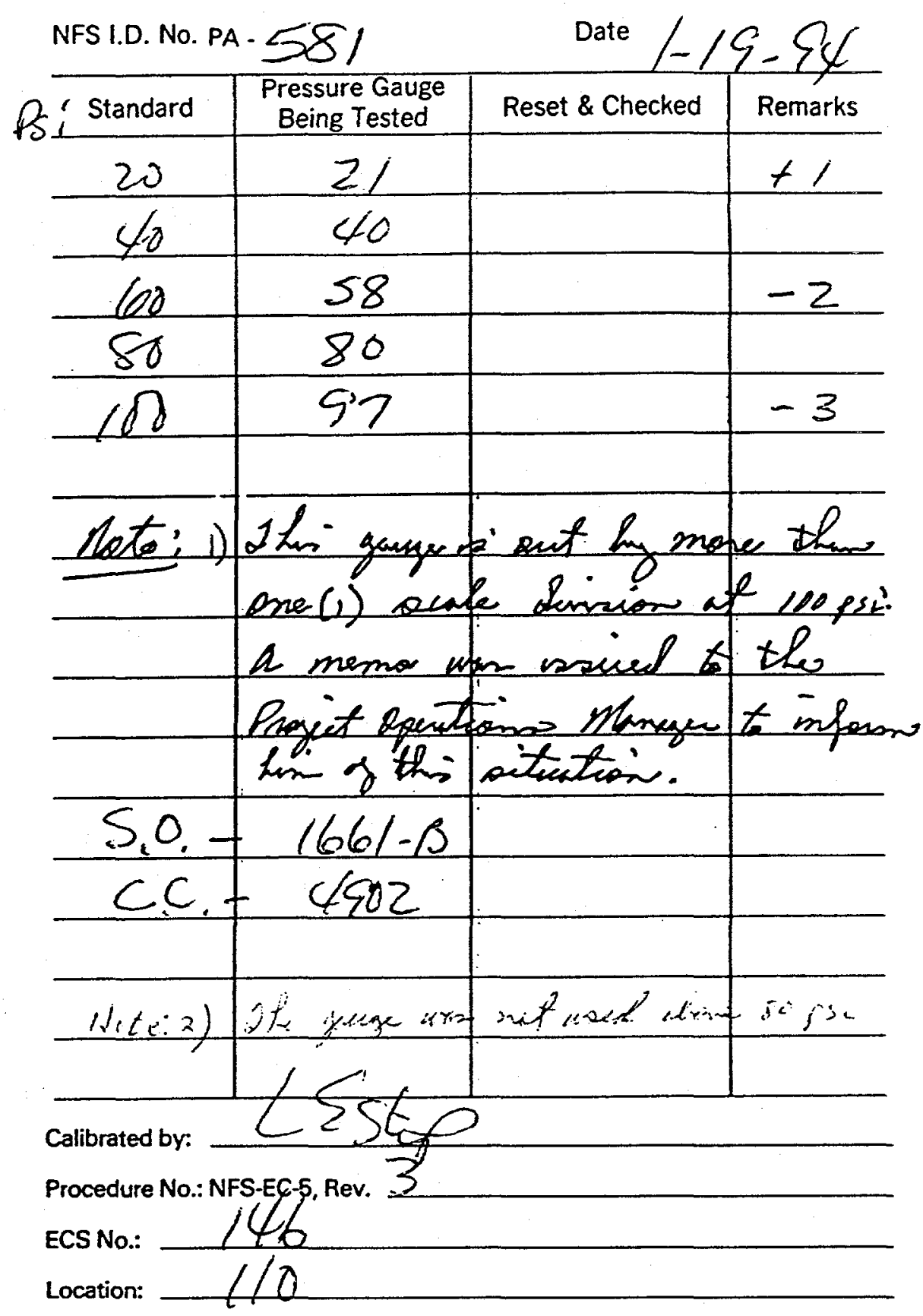

Knowingly or willfully falsifying or concealing a material fact on this form, or making false, fictitious or fraudulent stalements or representations herein could constitute a felony punishable under Federal Statutes. 
N.F. S-ID No $P A-582$

Description Pressure Gage

MODEL NO.

None

CALIBRATION FREQUENCY Amuially
MANUFACTURER TReble,

SERAAL No._ Honer

CALIBRATION PROCEDURE NO. EC - 5

ACCURACY $\pm 2,5 ;$

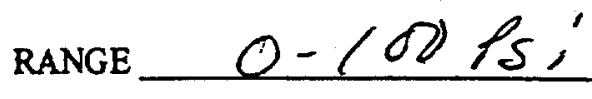

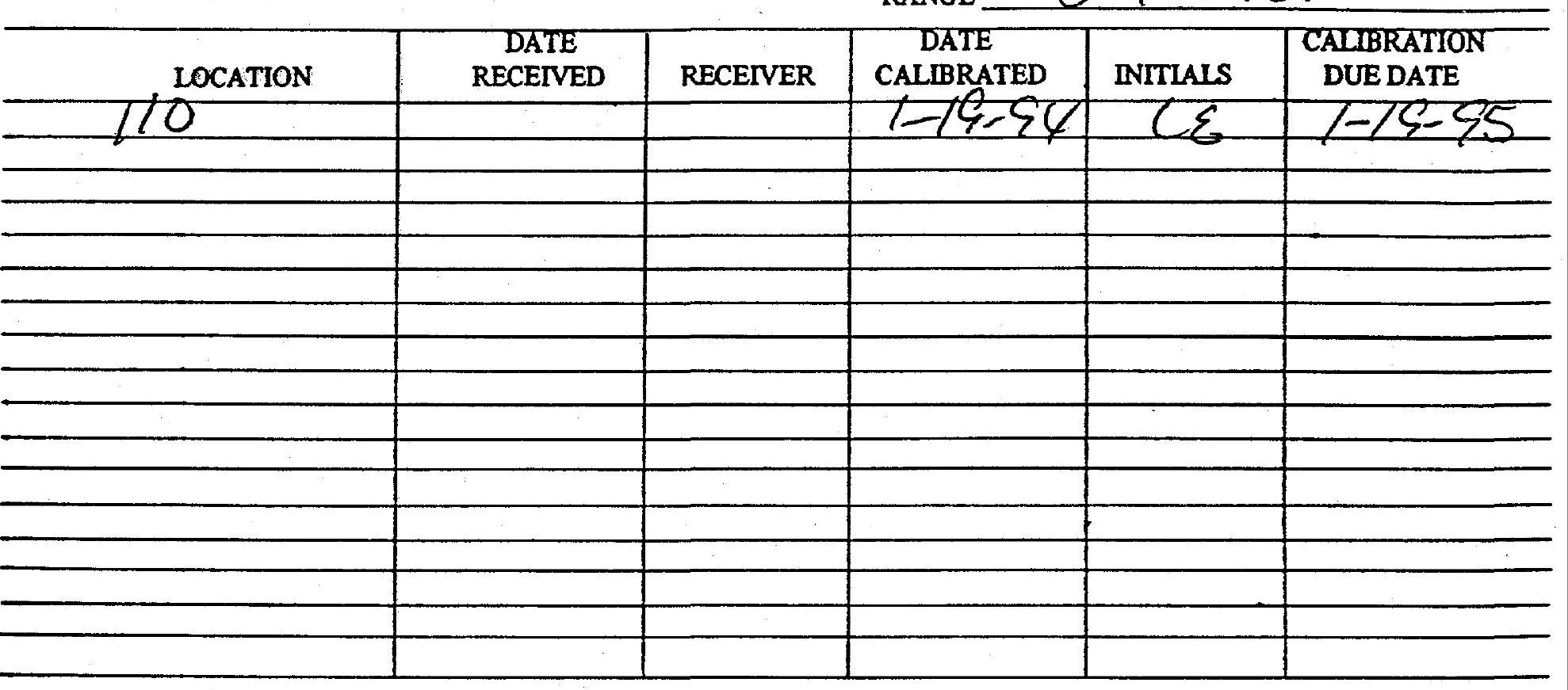


CALIBRATION OF PRESSURE GAUGES

Form C.6

NFS I.D. No. PA - 582

Date

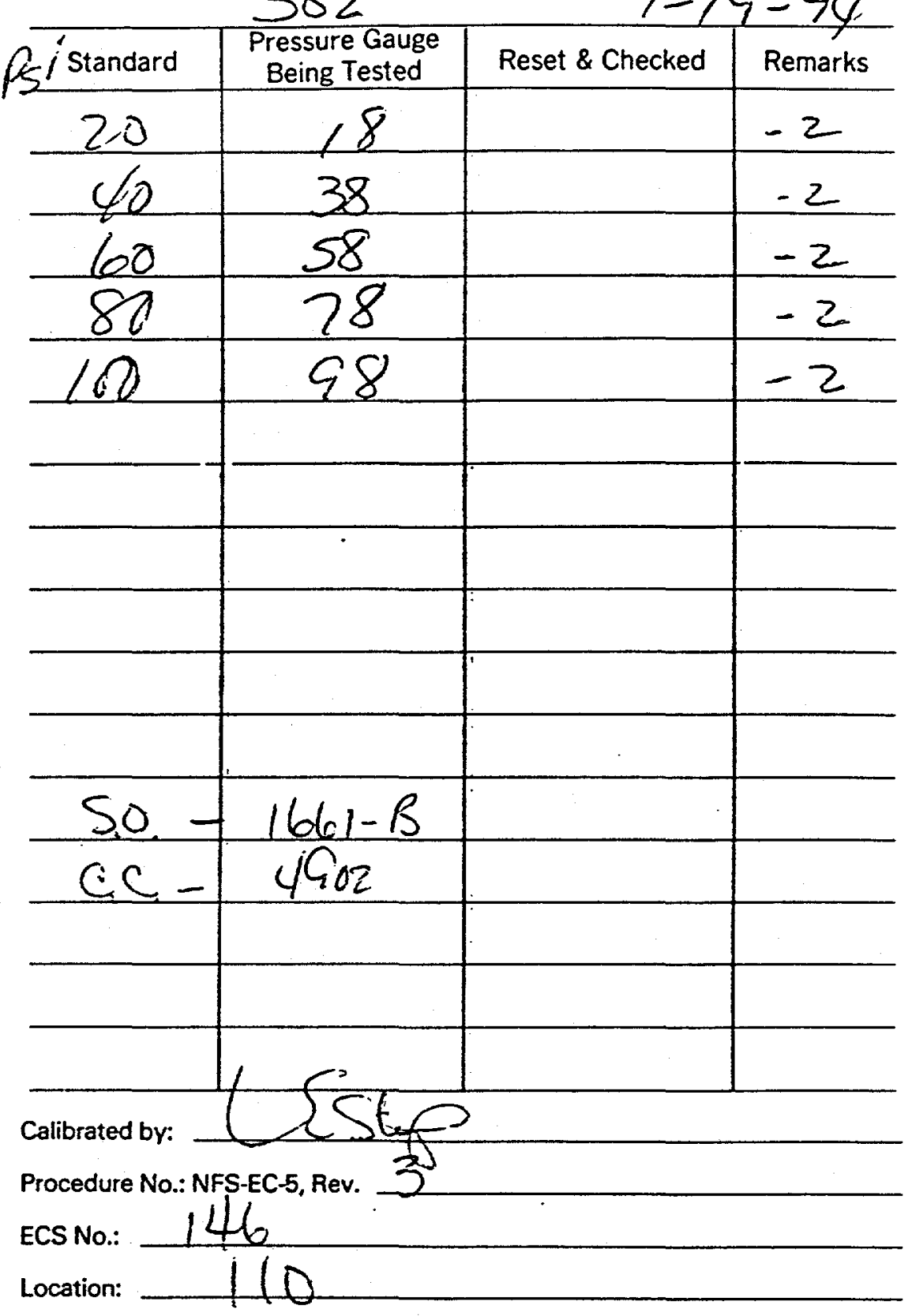

Knowingly or willfully falsifying or concealing a material fact on this form, or making false, fictitious or fraudulent statements or representations herein could constitute a felony punishable under Federal Statutes. 
N.F.S.- I.D. No. $P A-583$

MANUFACTURER Welkoles

Description PRessure Gauge SERIAL No. Ne ne

MODEL No. $615-104$ CALIBRATION PROCEDURE No. EC 5

CALIBRATION FREQUENCY ACCURACY

1 D sic

range O- 100 Ps ie

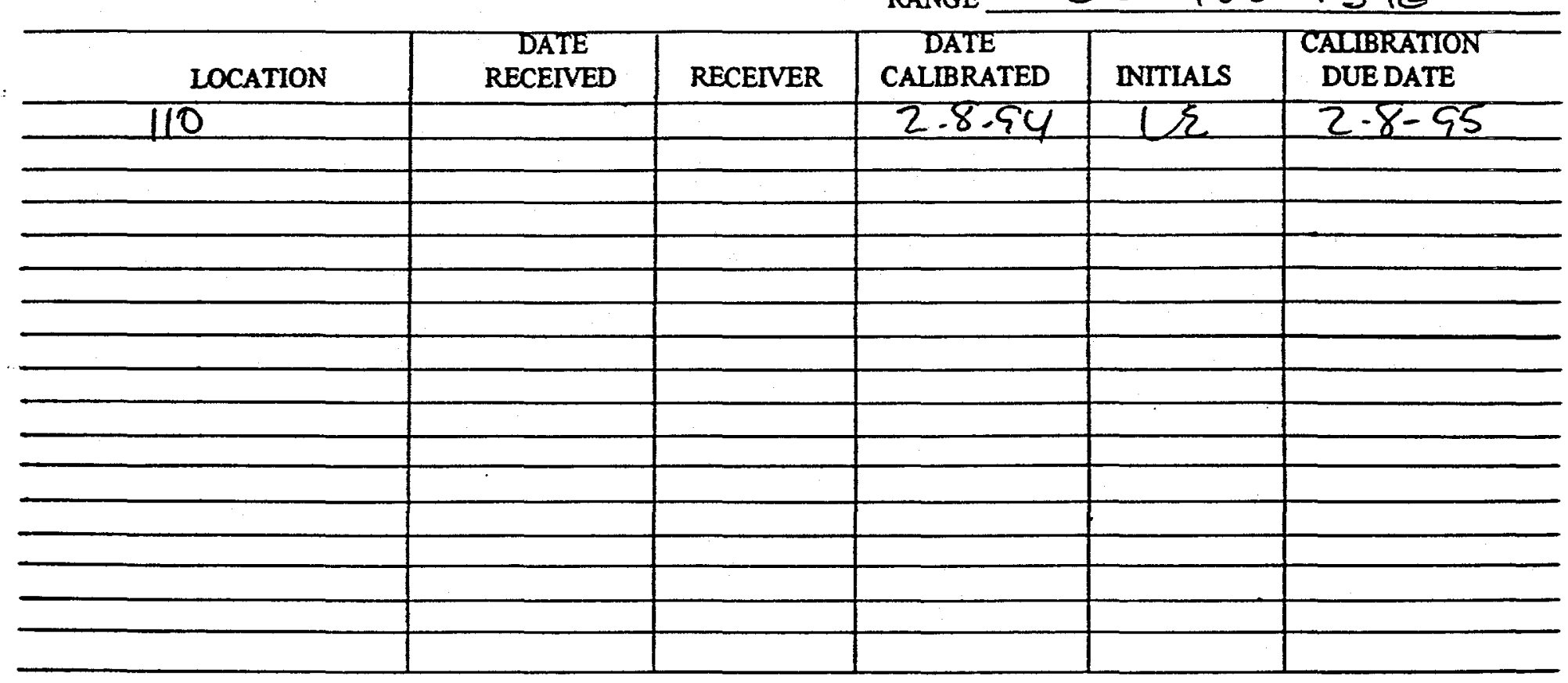


CALIBRATION OF PRESSURE GAUGES

NFS I.D. No. PA -583

Date $2 \cdot 8-94$

\begin{tabular}{|c|c|c|c|}
\hline Standard & $\begin{array}{c}\text { Pressure Gauge } \\
\text { Being Tested }\end{array}$ & Reset \& Checked & Remarks \\
\hline 20 & 20 & & \\
\hline 40 & 40 & & \\
\hline 60 & 60 & & \\
\hline 80 & 80 & & \\
\hline 100 & 100 & & \\
\hline & & 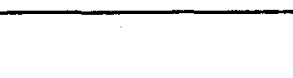 & \\
\hline & & & \\
\hline & & & \\
\hline & & & \\
\hline 50 & 600 & & \\
\hline QC & 1602 & & \\
\hline & & & \\
\hline & & & . \\
\hline ibrated by: & & & \\
\hline $\begin{array}{l}\text { ocedure No.: } \\
\text { S No.: }\end{array}$ & EC-5, Rev. 3 & & \\
\hline cation: & & & \\
\hline
\end{tabular}

Knowingly or willfully falsifying or concealing a material fact on this form, or making false, fictitious or fraudulent statements or representations herein coulo constifute a felony punishable under Federal Statutes. 
N.F.S.- I.D. No. TA- 877

Description temp. Indicator

MoDel No. None

CALIBRATION FREQUENCY Annually
MANUFACTURER Treníl Serial No. None

CALIBRATION PROCEDURE No. $\varepsilon C \cdot 10$ ACCURACY \pm 2 OF

RANGE $30-240^{\circ}$

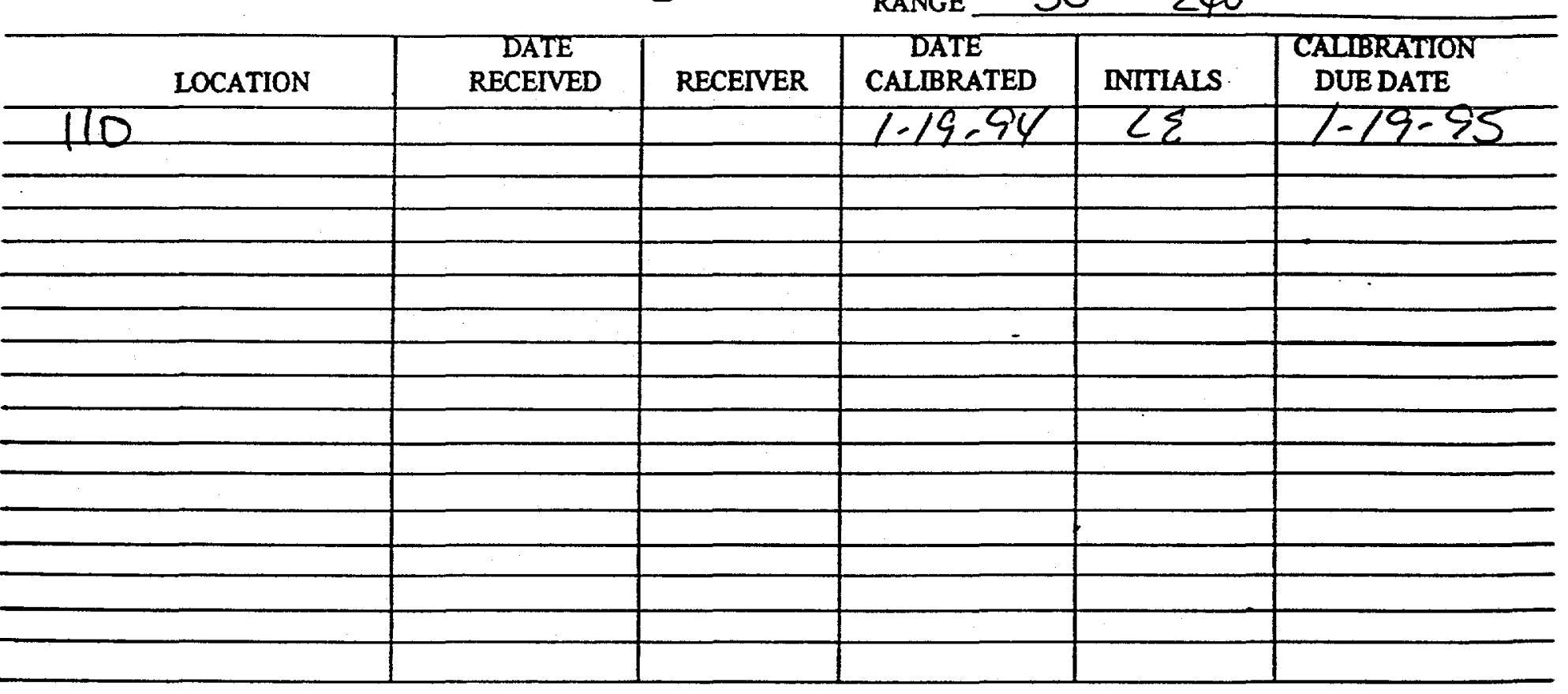


CALIBRATION OF TEMPERATURE INDICATORS

Form $\mathrm{C}^{-4}$

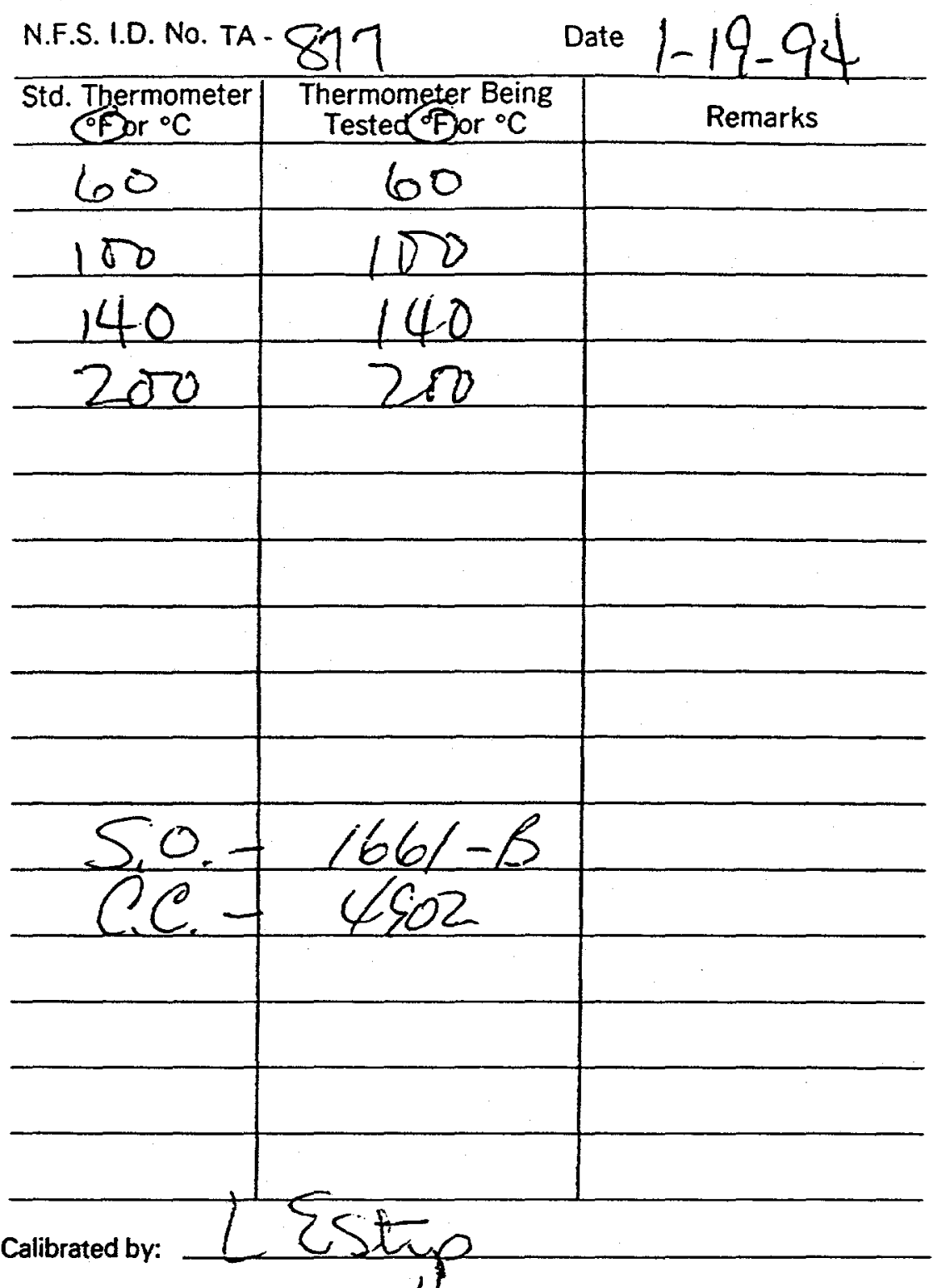

Procedure No.: NFS-EC-10, Rev. 4

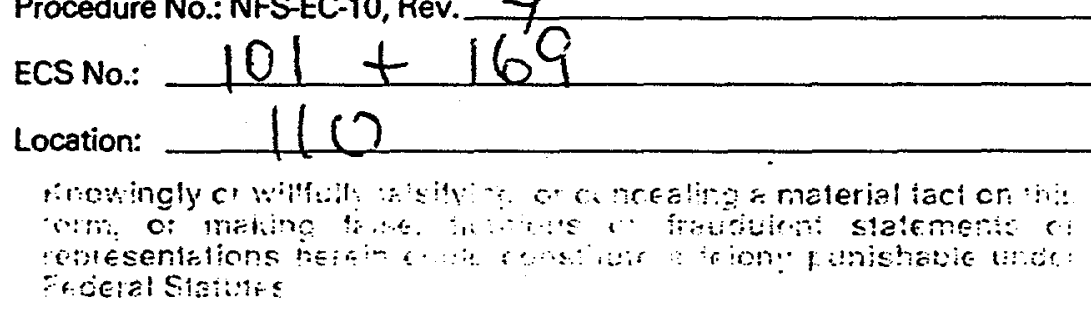


N.S. I.D. No. TA. 878

MANUFACTURER Iheriles

Description Temp. Indicator serial no. Nome

MODEL NO.

CALIBRATION PROCEDURE NO. $E C-10$

calibration frequency Annually

ACCURACY \pm 2 of

RANGE $30-240^{\circ}$

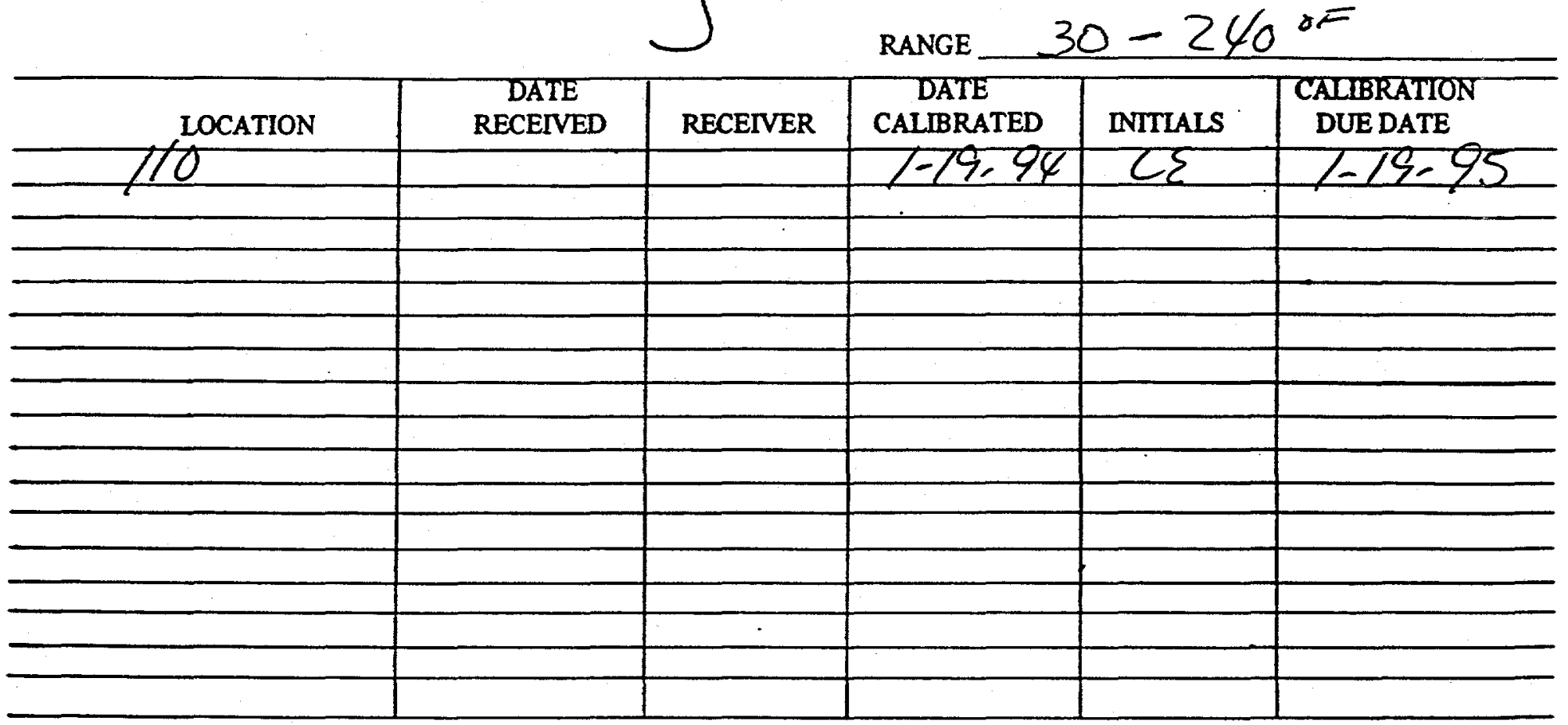




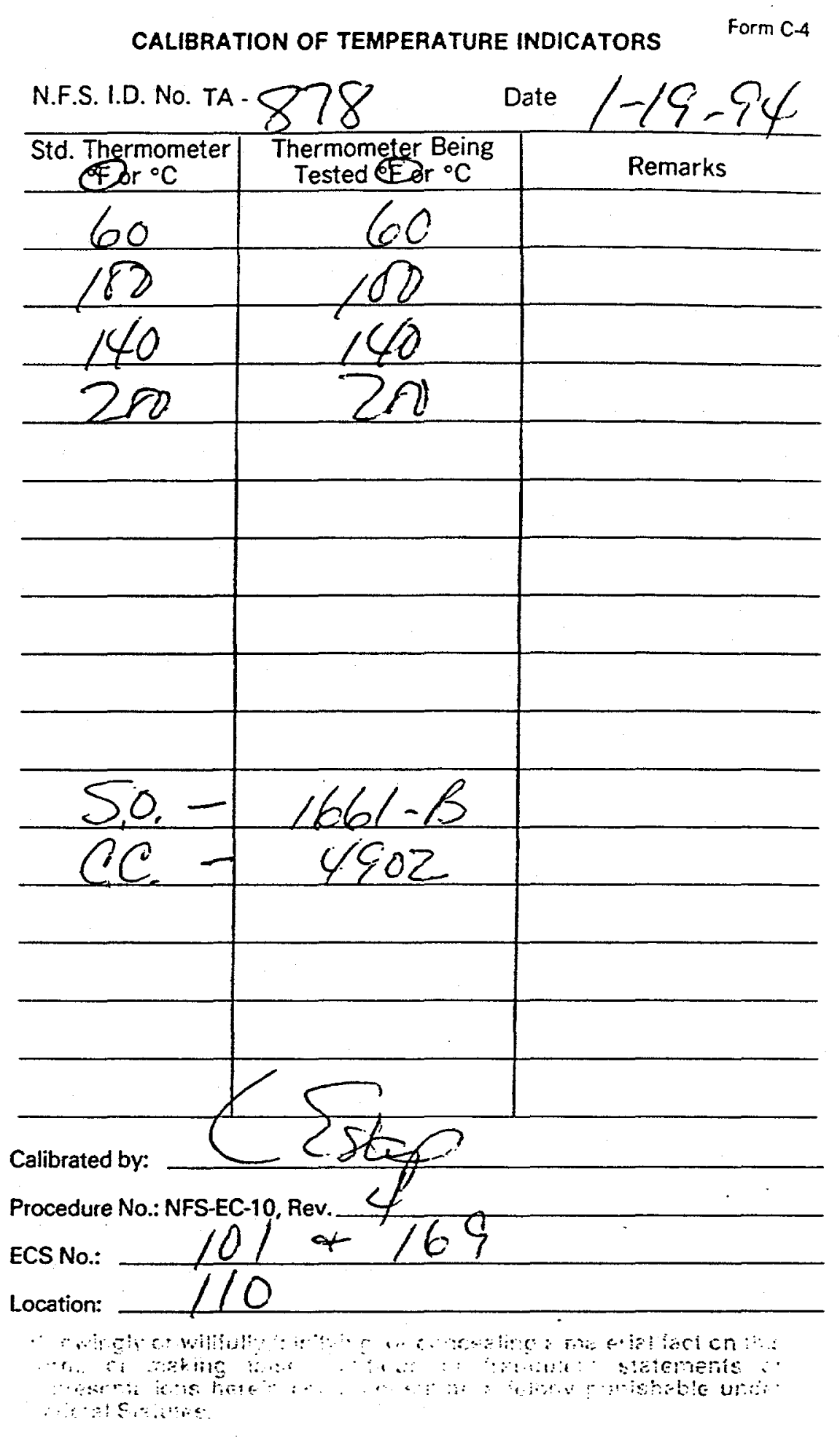


N.F.S. I.D. No. TA- 879

MANUFACTURER Throes

DESCRITIONTemp Indicator SERIAL NO.

None

MODEL NO.

CALIBRATION PROCEDURE No. $E C-10$

caliBRATION FREQUency Annually

ACCURACY $\pm 2^{\circ} \mathrm{F}$

RANGE $30-240^{\circ} \mathrm{F}$

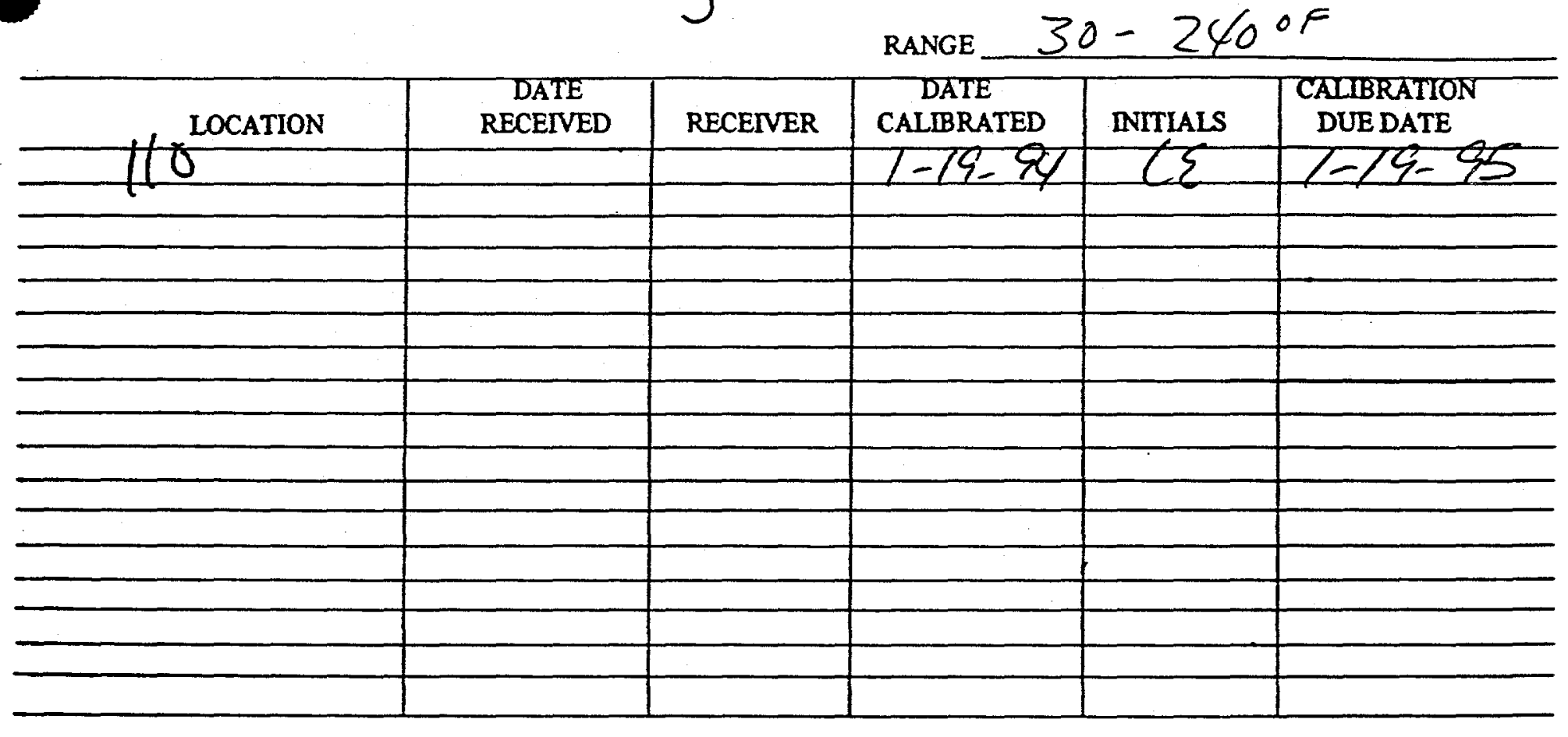


N.F.S. I.D. No. $7 A-880$

Description temp. Indicator

model No._ Pone

calibration frequency Anmudely
MANUFACTURER Therese

serial no. Nome

CALIBRATION PROCEDURE NO. $E C-10$

ACCURACY \pm 2 OF

RANGE $30-240^{\circ} \mathrm{F}$

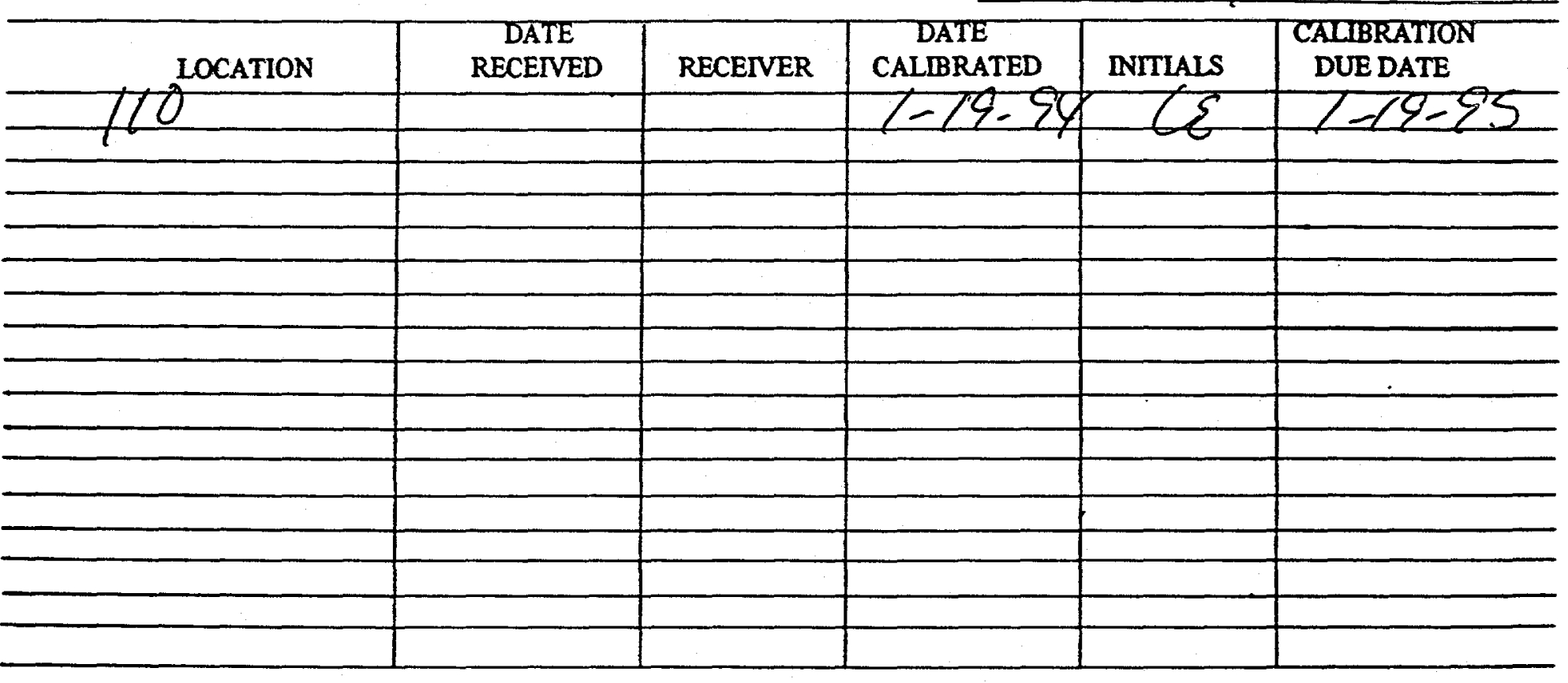


N.F. I.D. No. IA- 88 DESCRPTION temp INdícator, moDel No. Dlone CALIBRATION FREQUENCY

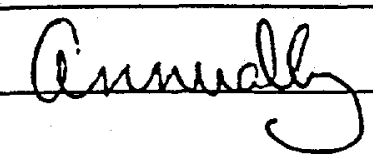

MANUFACTURER

SERIAL NO. Nose

CALIBRATION PROCEDURE No. $S \mathrm{C}-10$ ACCURACY \pm 2 OF

RANGE $30-240$ OF DATE $1-19-90$

\begin{tabular}{c} 
INITIALS \\
\hline$\varepsilon$
\end{tabular}

CALIBRATION DUE DATE $1-19-95$ 
CALIBRATION OF TEMPERATURE INDICATORS

Form C-4

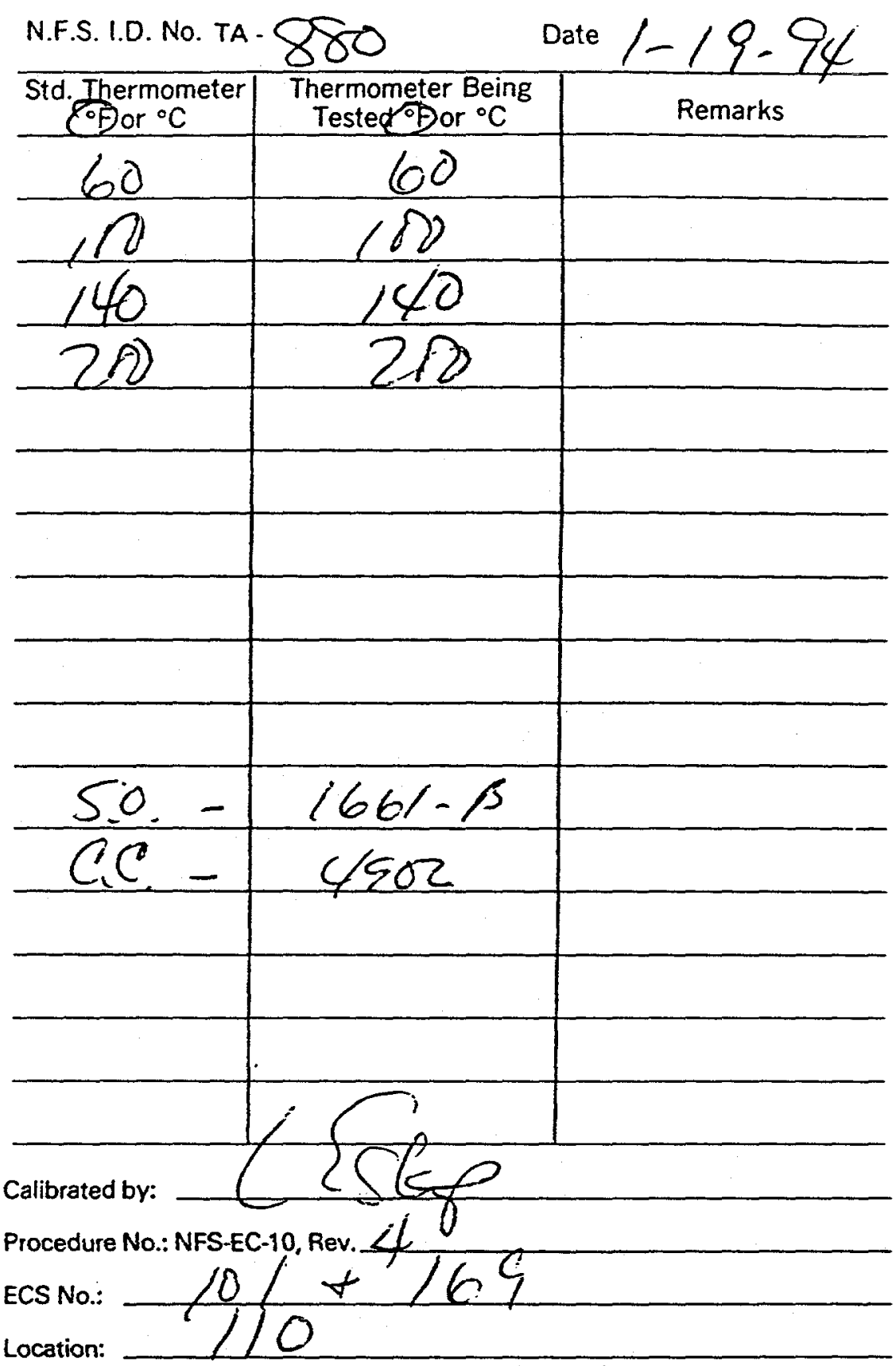

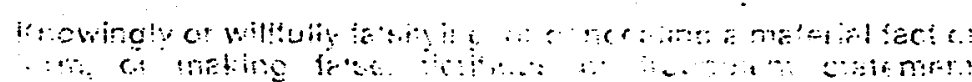

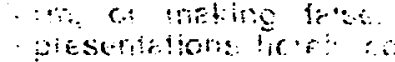

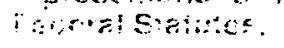


N.F.S. ID. NO. $=14-88$

MANUFACTURER IA L CC S

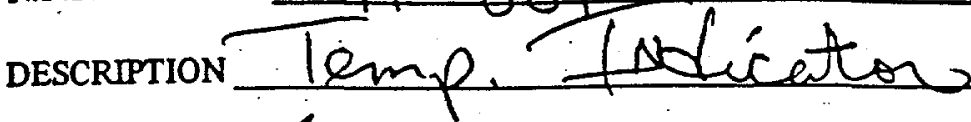
SERIAL NO. Hone MODEL No. Done CALIBRATION PROCEDURE No. $\& C-10$ CALIBRATION FREQUENCY

Gisurato ACCURACY $\pm 20 F$ RANGE $30-240 \%$

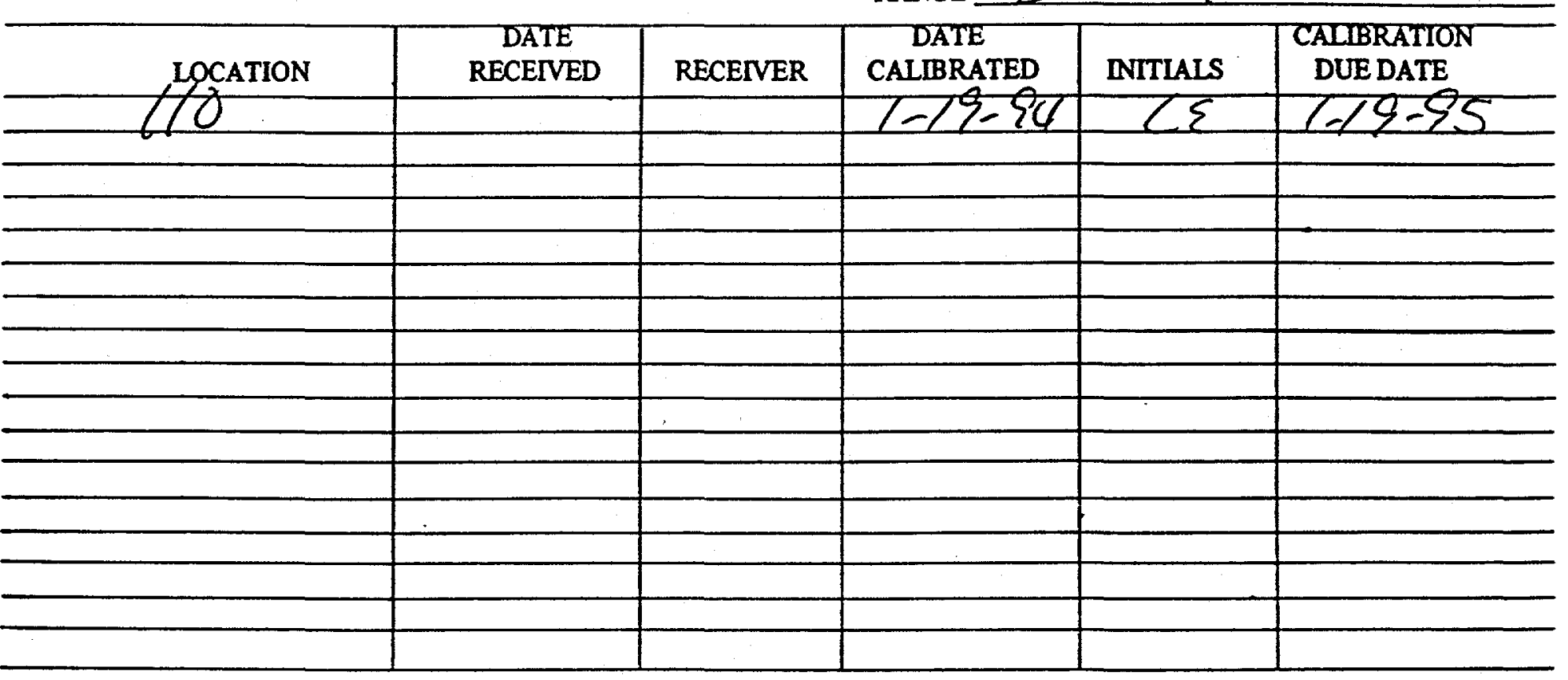


CALIBRATION OF TEMPERATURE INDICATORS

Form $\mathrm{C}-4$

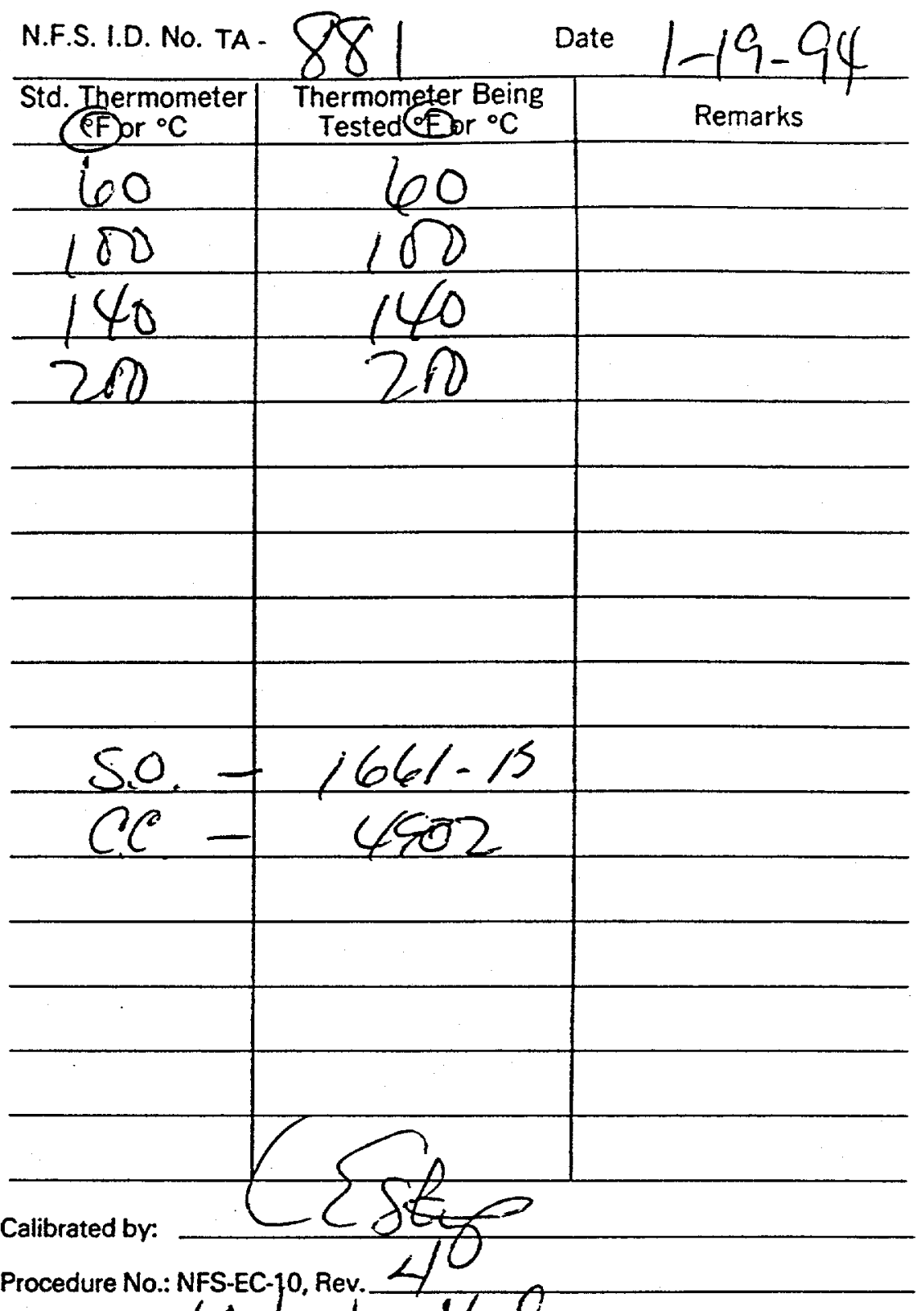

Procedure No.: NFS-EC-10, Rev. 10$\left.)^{2}+10\right\}$

Location: $1 / 0$

reowigy ot withuty

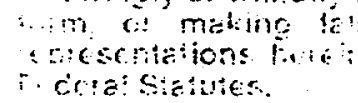




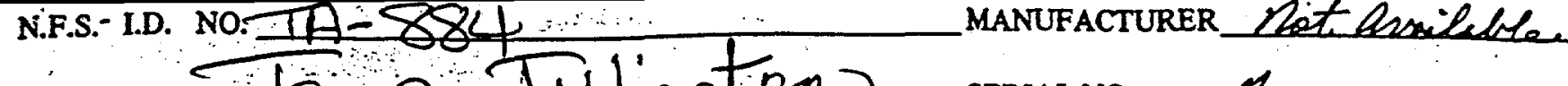

DESCRIPTION (emp Indicator) SERIAL No. Tone

MODEL NO. Hone

CALIBRATION PROCEDURE No. $\varepsilon C-10$

calibration frequency Causally ACCURACY 2 OF (Readability) RANGE 0 - $250^{\circ} \mathrm{F}$

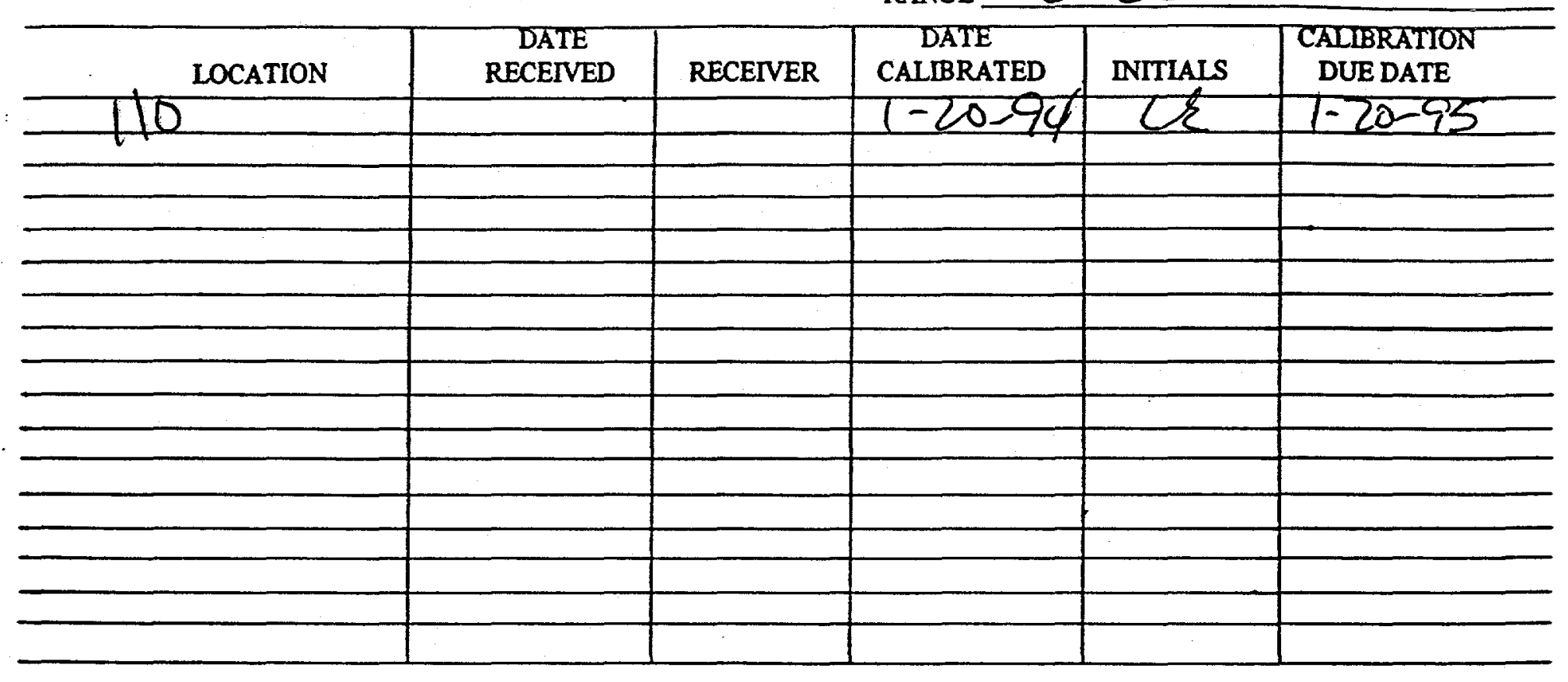


N.F.S. I.D. No. TA. 885

DESCRIPTION Temp. Indicator MODEL NO.

CALIBRATION FREQUENCY Onmula
MANUFACTURER Peotemo o SERIAL No. None CALIBRATION PROCEDURE No. $\varepsilon c-10$ ACCURACY $100 \%$

RANGE $200-1000 \partial$

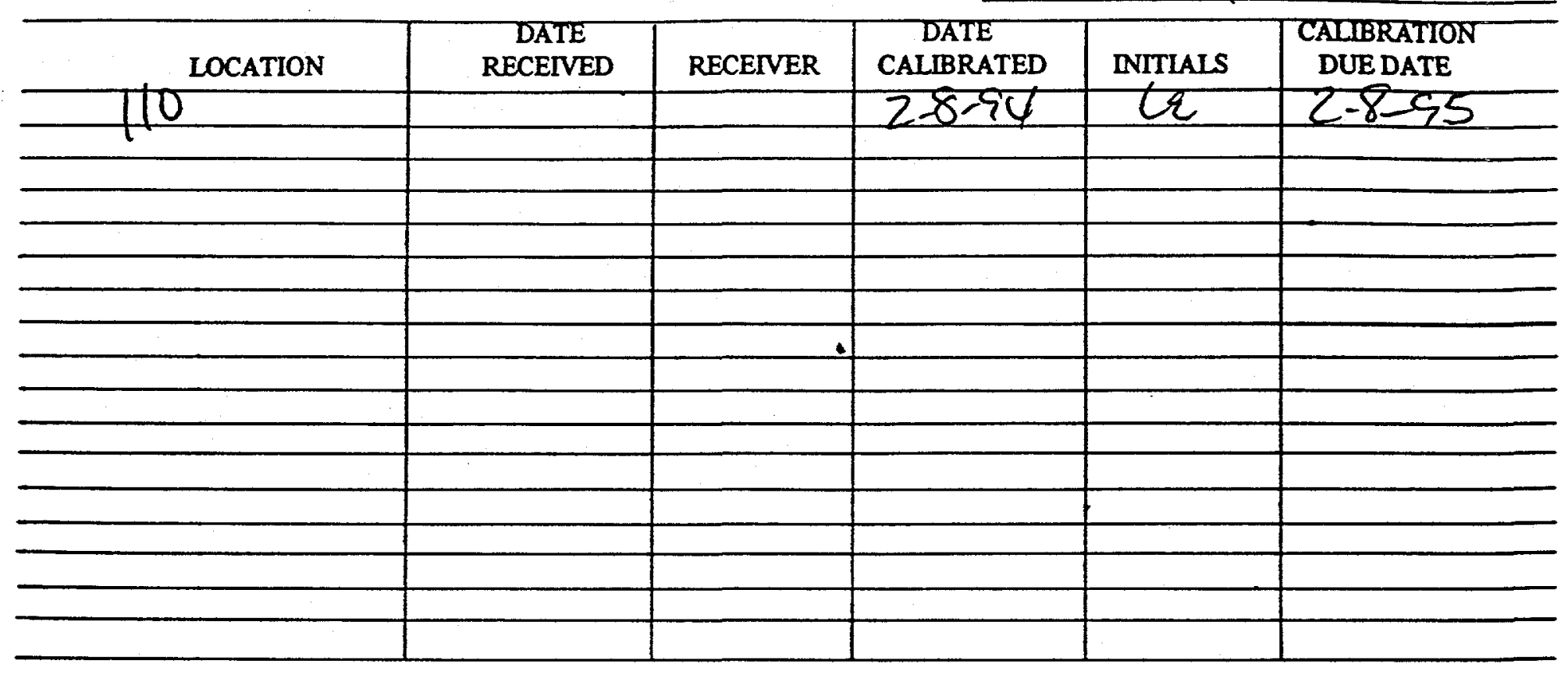




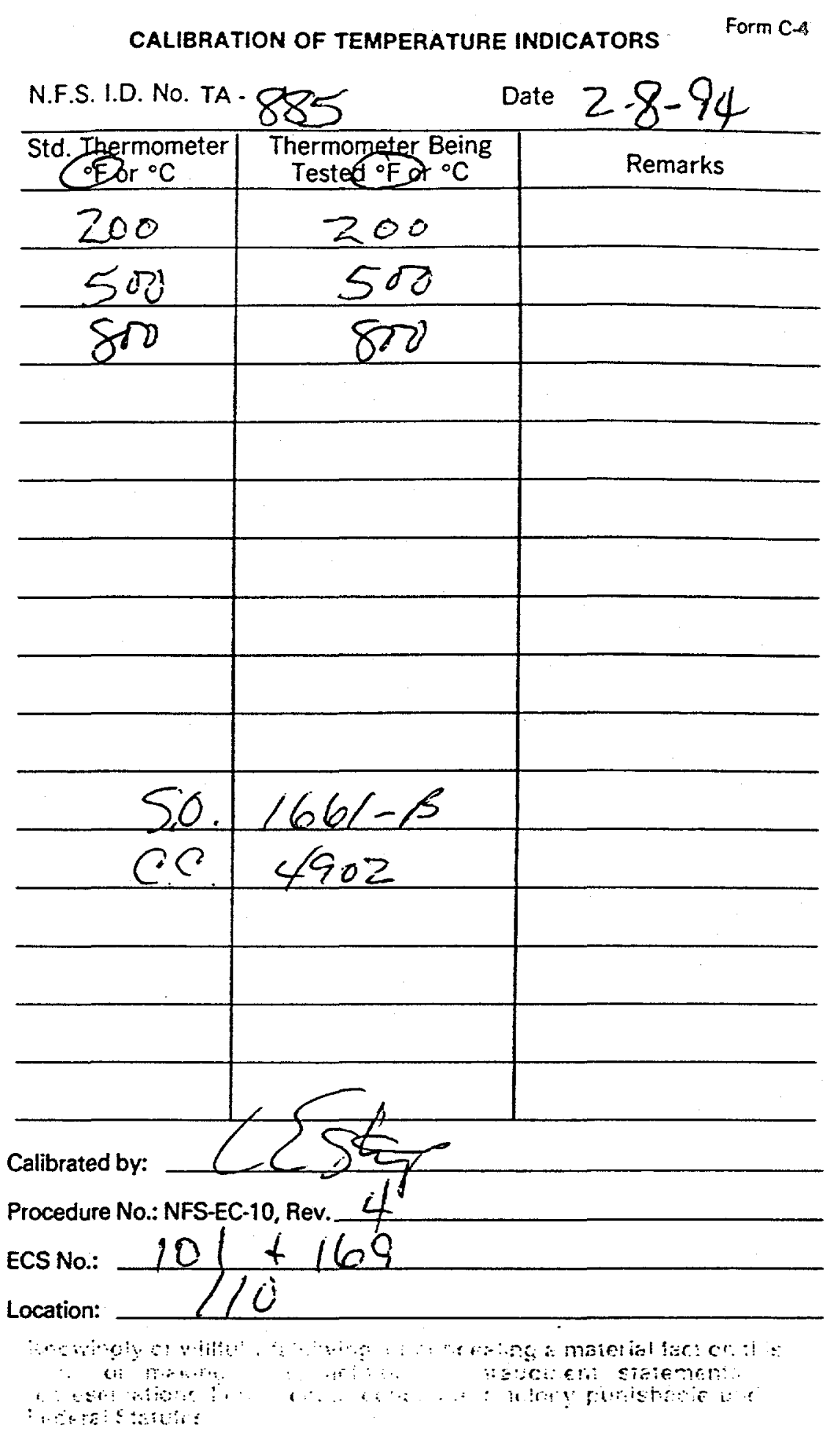


N.F.S. I.D. No. $T$ H- 886

MANUFACTURER totem 0 DESCRIPTION Temp Indicinton SERIAL No. None MODEL NO. CALIBRATION PROCEDURE NO. SC 10 CALIBRATION FREQUENCY Minimally aCCURACY $100 \mathrm{~F}$ RANGE $280-10070^{\circ}$

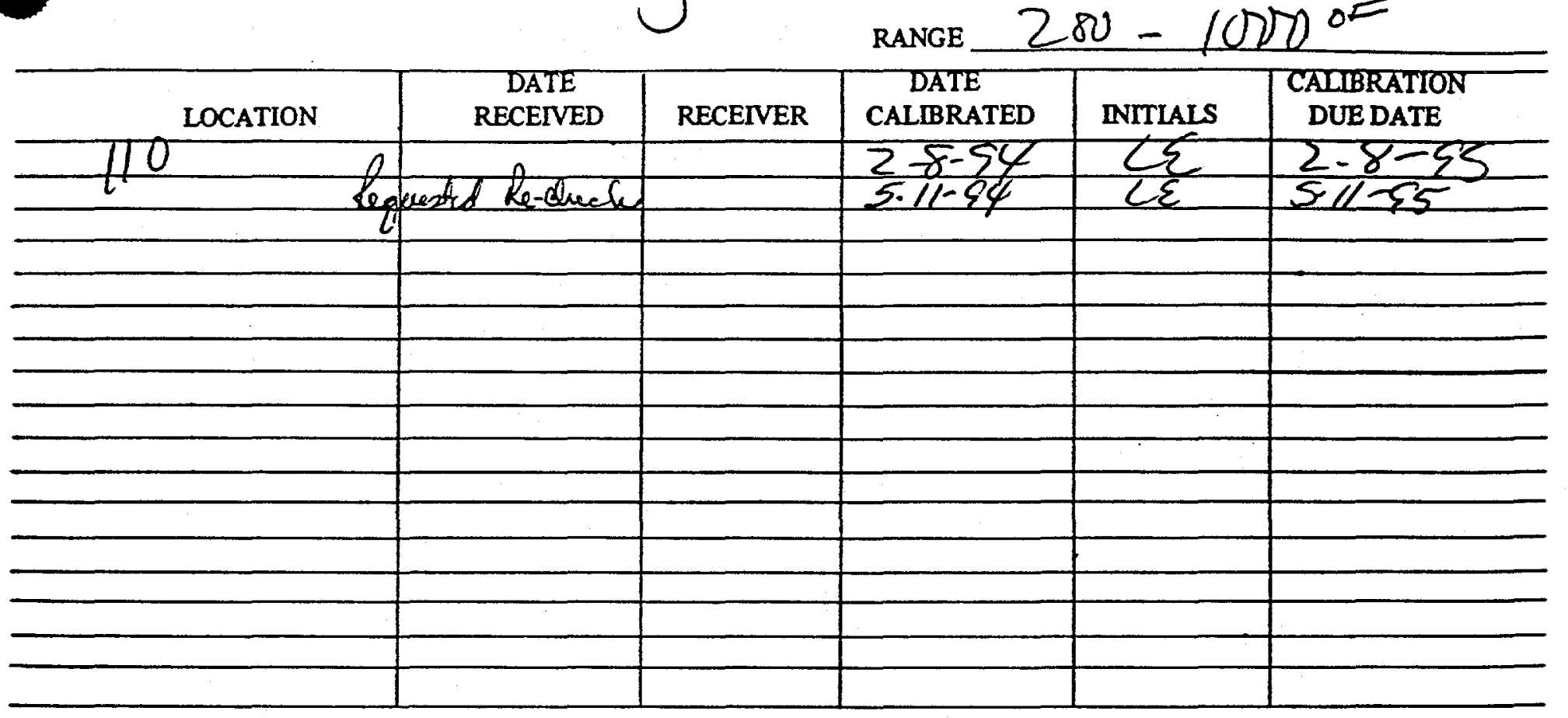


CALIBRATION OF TEMPERATURE INDICATORS Form C-4

N.F.S. I.D. No. TA. 886 Date $5.11-94$

\begin{tabular}{c|c|c}
\hline $\begin{array}{c}\text { Std. Thermometer } \\
\text { (o for }{ }^{\circ} \mathrm{C}\end{array}$ & $\begin{array}{c}\text { Thermometer Being } \\
\text { Tested }{ }^{\circ} \mathrm{C}\end{array}$ & Remarks \\
\hline 200 & 260 & \\
\hline 502 & 500 & \\
\hline 810 & 872 & \\
\hline & & \\
\hline & & \\
\hline & & \\
\hline
\end{tabular}

to redux remuicel.

\begin{tabular}{l|l|l}
\hline & & \\
\hline & & \\
\hline & & \\
\hline & & \\
\hline Calibrated by: & & \\
\hline
\end{tabular}

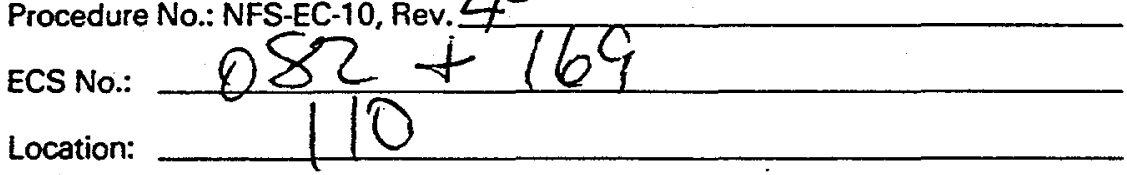

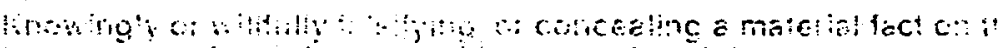

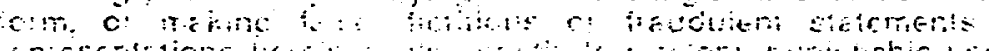

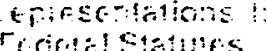


CALIBRATION OF TEMPERATURE INDICATORS FOrm C-4

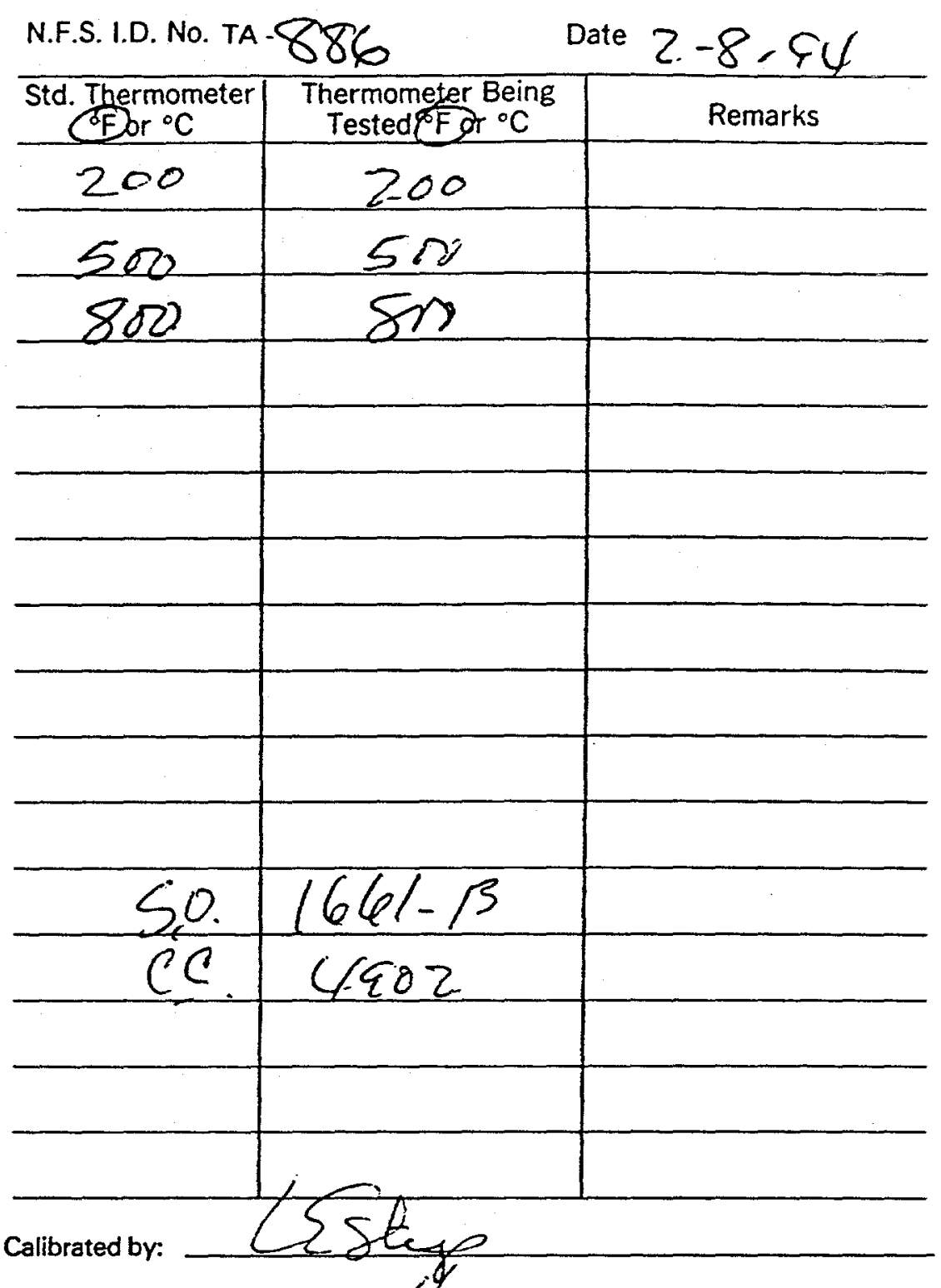

Procedure No.: NFS-EC-10, Rev. $\frac{\text { if }}{4}$

ECSNo.: $10 / \% 16 ?$

Location: $1 / 0$

frewongly or wile

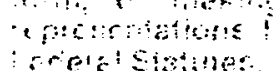


N.F.S ID. NO A. 890

MANUFACTURER Tel $=T_{R} y$

DESCRIPTON Temp IN diction ( $D_{i}$ il) SERIAL No.

MODEL NO

CALIBRATION PROCEDURE NO. $\varepsilon C-16$

CALIBRATION FREQUENCY Ammerally

ACCURACY \pm 10 F

RANGE 200 to 1000 of

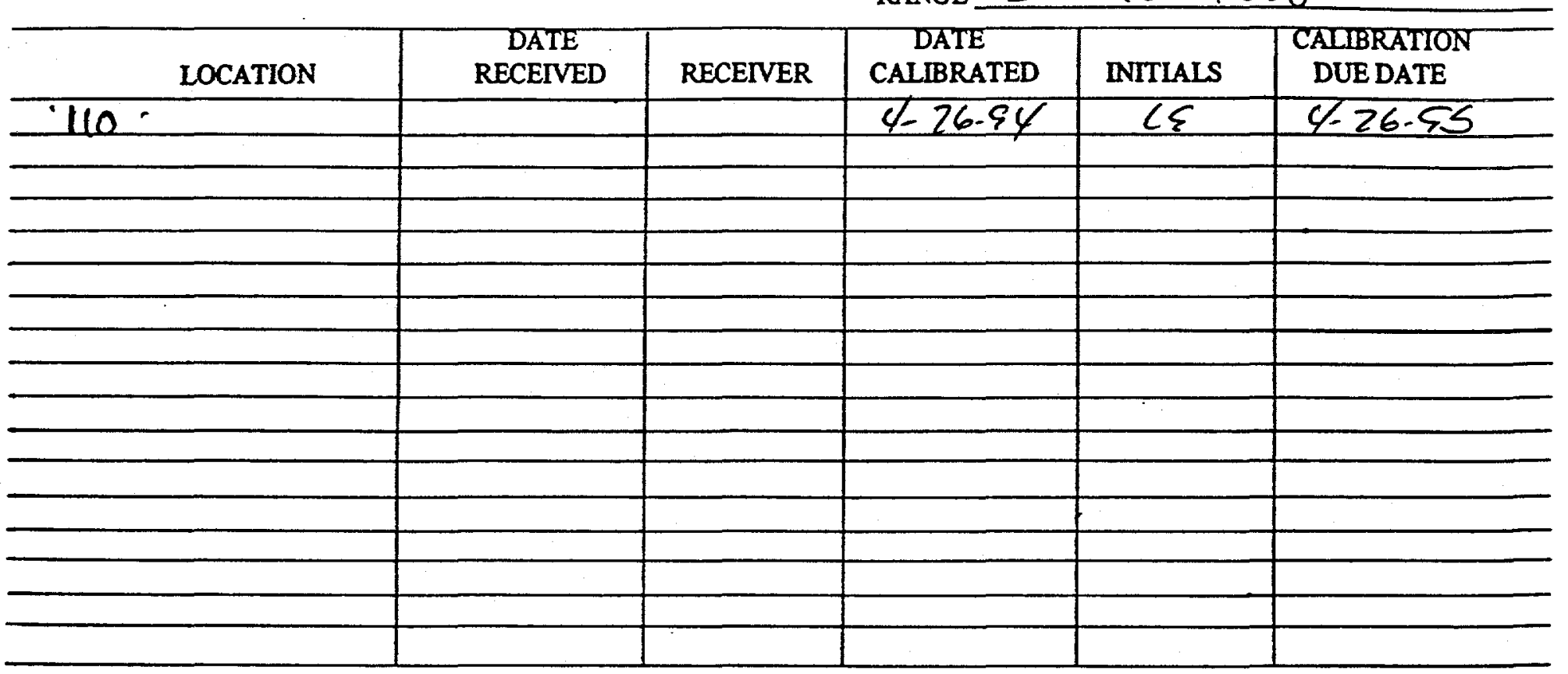


CALIBRATION OF TEMPERATURE INDICATORS Form C.4

\begin{tabular}{c|c|c} 
N.F.S. I.D. No. TA. 890 & Date $4-26.54$ \\
\hline $\begin{array}{c}\text { Std. Thermometer } \\
\text { For }{ }^{\circ} \mathrm{C} C\end{array}$ & $\begin{array}{c}\text { Thermometer Being } \\
\text { Tested E or }{ }^{\circ} \mathrm{C}\end{array}$ & Remarks \\
\hline 200 & 200 & \\
\hline 300 & 300 & \\
\hline 400 & 400 & \\
\hline 500 & 500 & \\
\hline 600 & 60 & \\
\hline 700 & 20 & \\
\hline 800 & 800 & \\
\hline & & \\
\hline & & \\
\hline & & \\
\hline & & \\
\hline & & \\
\hline & & \\
\hline & & \\
\hline & & \\
\hline & & \\
\hline
\end{tabular}

Calibrated by: $\quad \sum 54$

Procedure No.: NFS-EC-10, Rev.

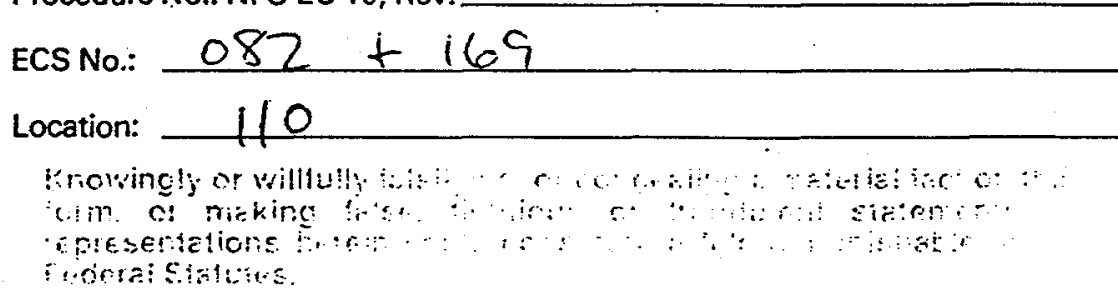


CALIBRATION OF THERMOCOUPLES

Form C-5

Due Date $4-26-85$

Accuracy $\pm 4^{\circ \mathrm{F}}$

Procedure No.: NFS-EC-20, Rev. 4

NFS I.D. No. SE- $3708 \quad$ Date $4=26-5 \%$

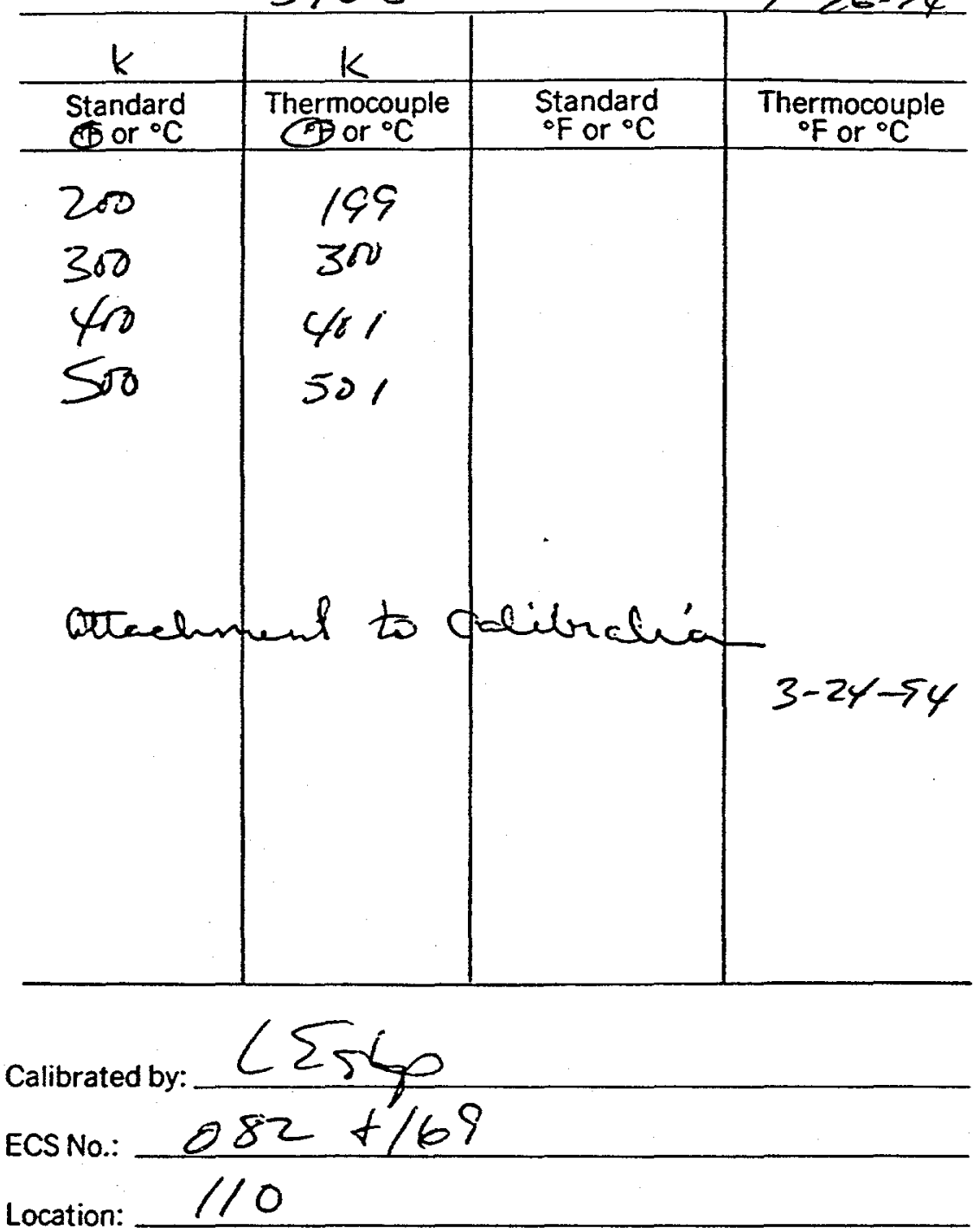

Knowingly or willfully falsifying or concealing a material fact on this form, or making false, fictitious or fraudulent statements or representations herein could constitute a felony punishable under Federal Statutes. 
CALIBRATION OF THERMOCOUPLES

Form C-5

Due Date 324-\$5

Accuracy \pm 4 of or $0.75 \%$ of reading (which ever is greater)

Procedure No.: NFS-EC-20, Rev. 4

NFS I.D. No. SE - 3788

$3-25-94$

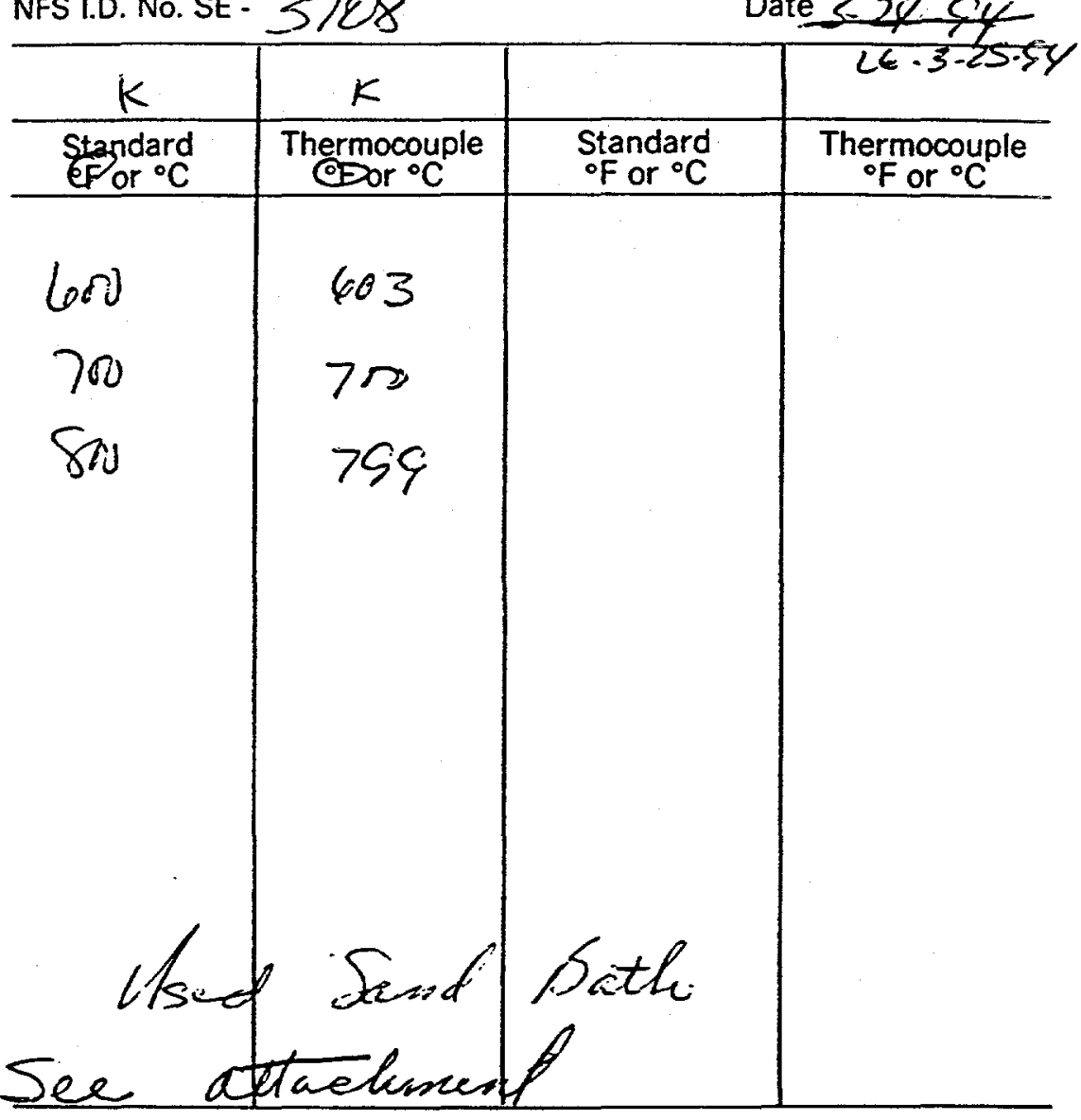

Calibrated by: $\frac{C \sqrt{56}}{082+169}$

Location: 110

Knowingly or willfully falsifying or concealing a material fact on this form, or making false, fictitious or fraudulent statements or representations herein could constitute a felony punishable under Federal Statutes. 
- CALIBRATION OF tHERMOCOUPLES

Form C.5

Due Date $4-26-15$

Accuracy $\pm 4^{\circ}$ r

Procedure No.: NFS-EC-20, Rev. 4

NFS I.D. No. SE - -3710

Date $4.2 \sin 4$

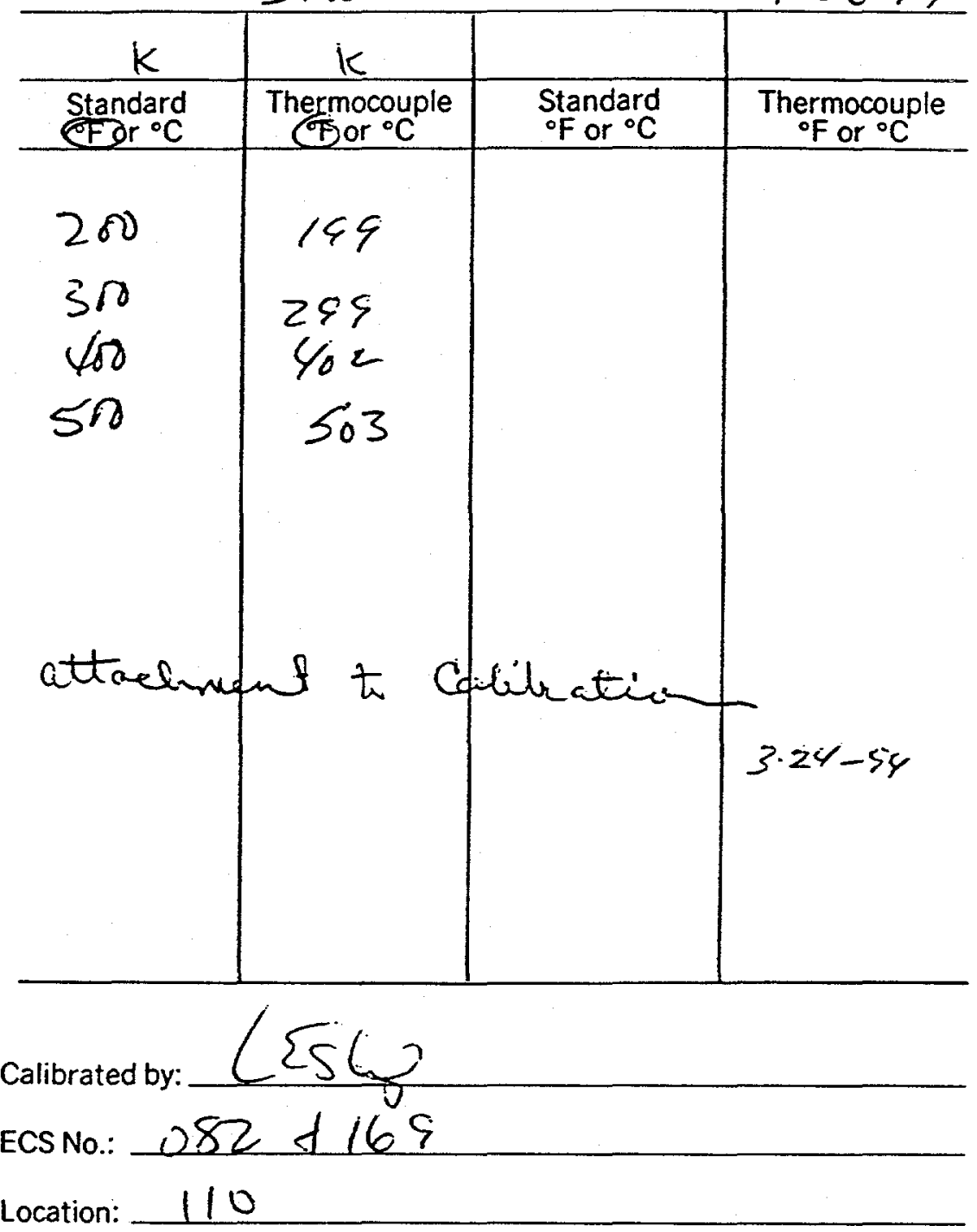

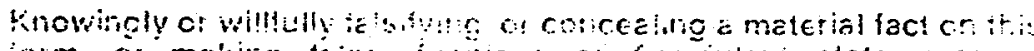

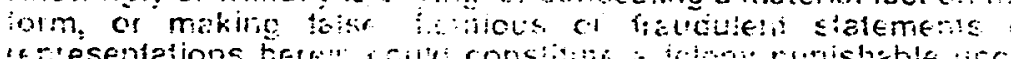

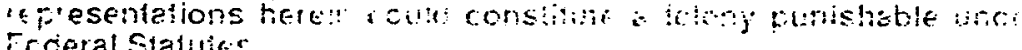
Trceral Stander 
- CALIBRATION OF THERMOCOUPLES

Form C-5

Due Date $3-24<5$

Accuracy $\pm 40 \mathrm{~F}$ or $\pm 0.75 \%$ of reading (Which ever is

Procedure No.: NFS-EC-20, Rev. 4 greater)

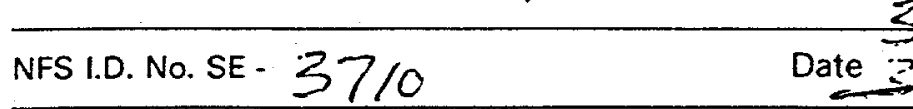

$3-25-94$

NFS I.D. No. SE - $37 / 0$

$\frac{24-94}{143-25 \cdot 54}$

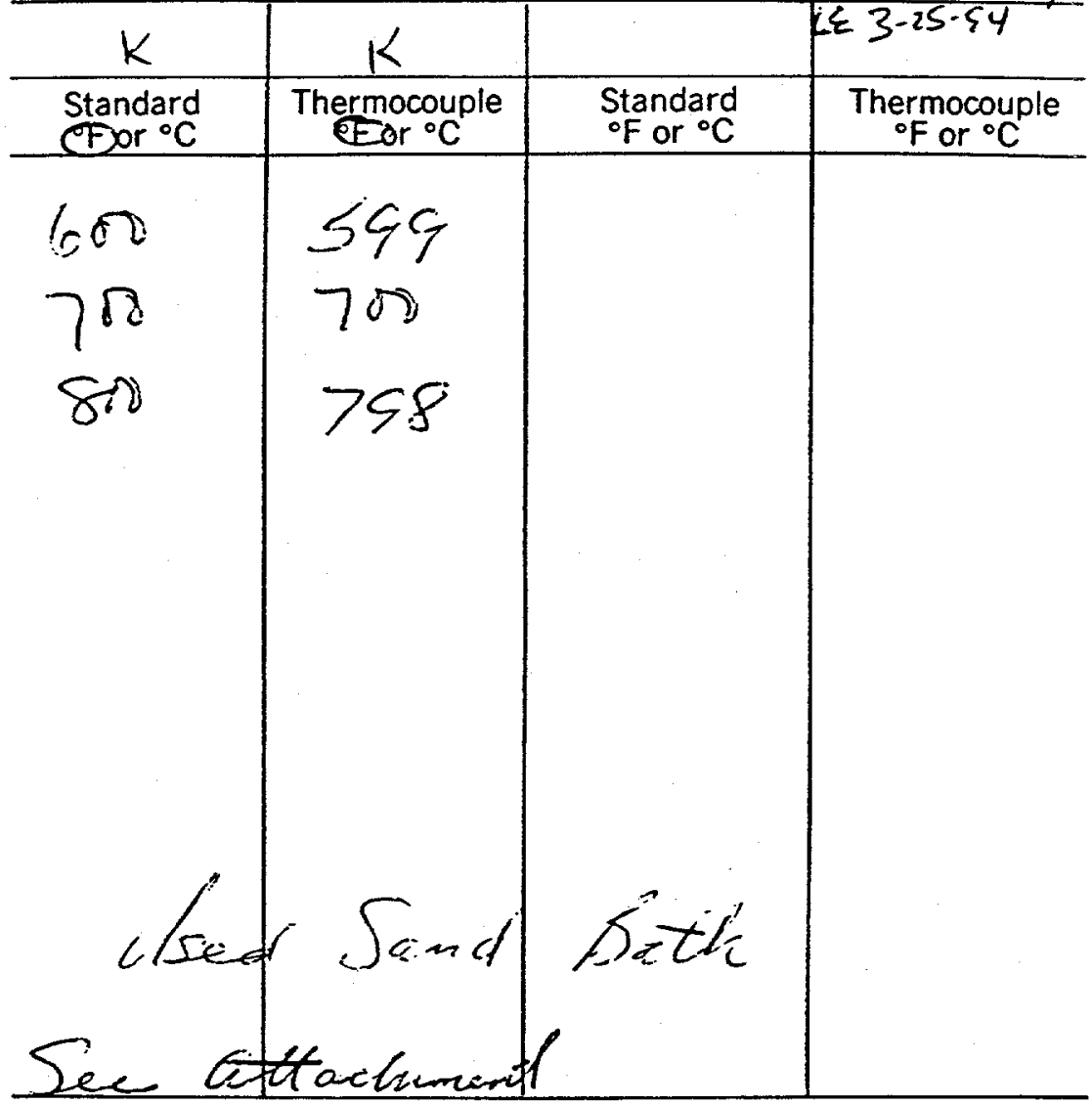

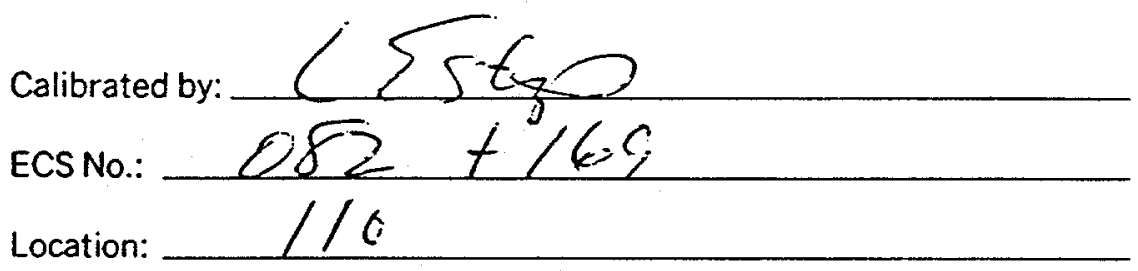

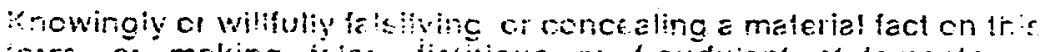

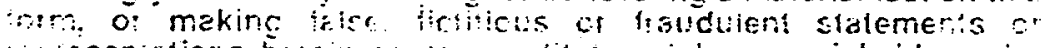

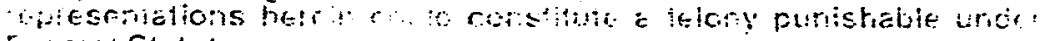

rquelat Statutes 
CALIBRATION OF THERMOCOUPLES

Form C.5

Due Date $3-2455$

Due Date $3-24>S$
Accuracy \pm .0F or $\pm 0.75 \%$ of reading (which ever i
procedure No.: NFS-EC-20, Rev. 4

$3-25.54$

NFS I.D. No. SE. $37 / /$

Date

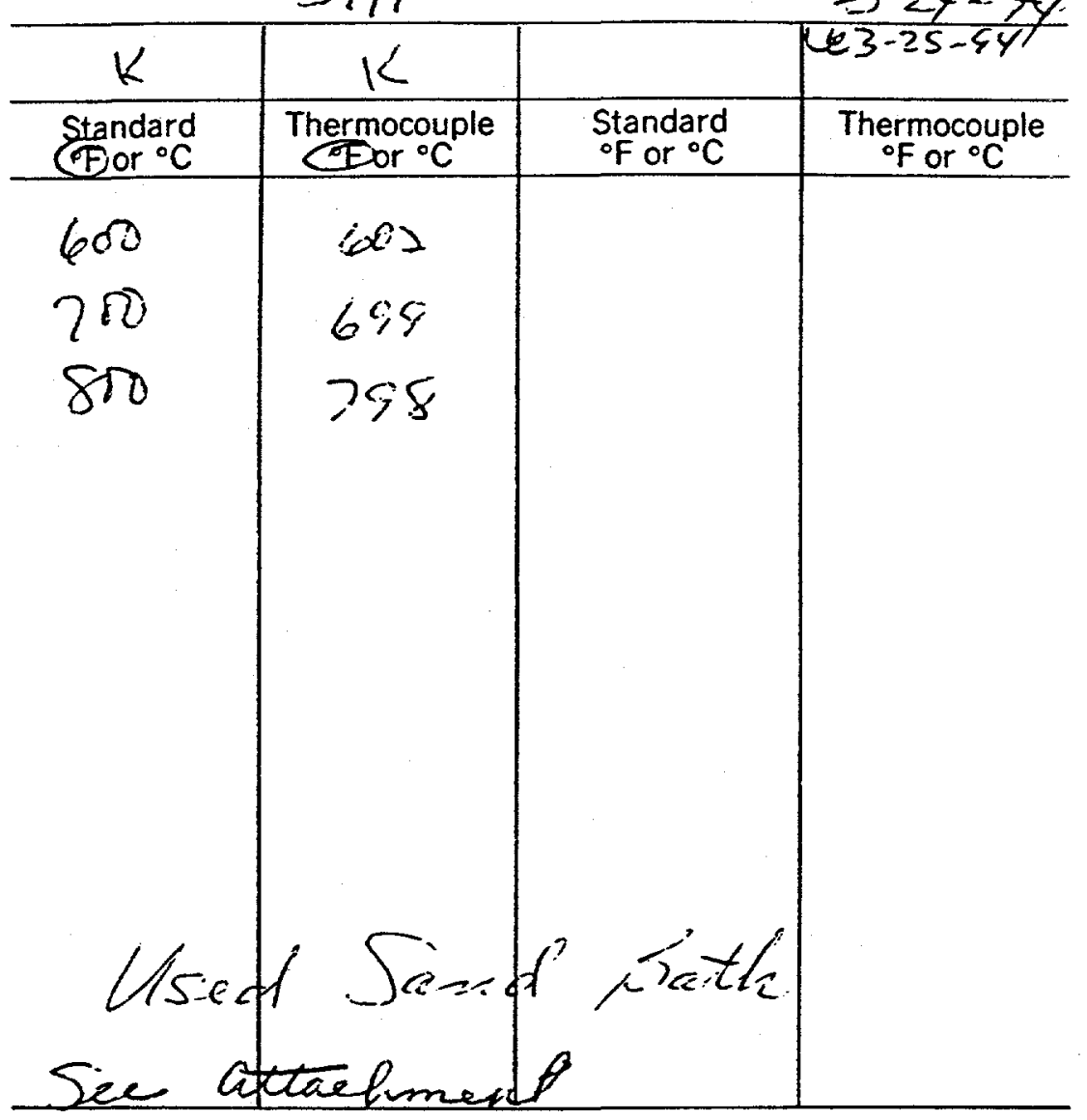

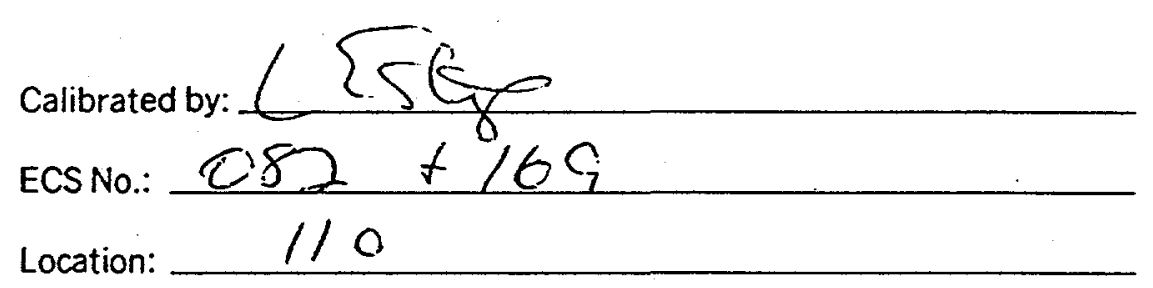

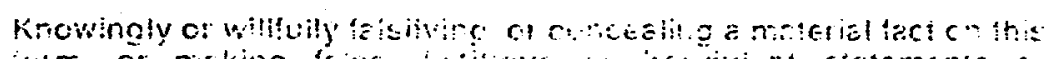

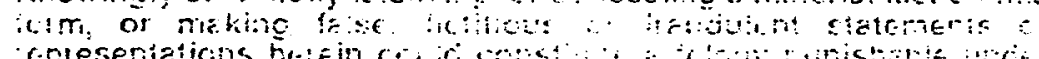

Federal Statutes. 
CALIBRATION OF THERMOCOUPLES

Form $\mathrm{C}-5$

Due Date $4-26-94$

Accuracy \pm ace $\pm \mathscr{L}^{\circ} \mathrm{F}$

Procedure No.: NFS-EC-20, Rev. 4

NFS I.D. No. SE - $37 / \quad$ Date $4-2458$

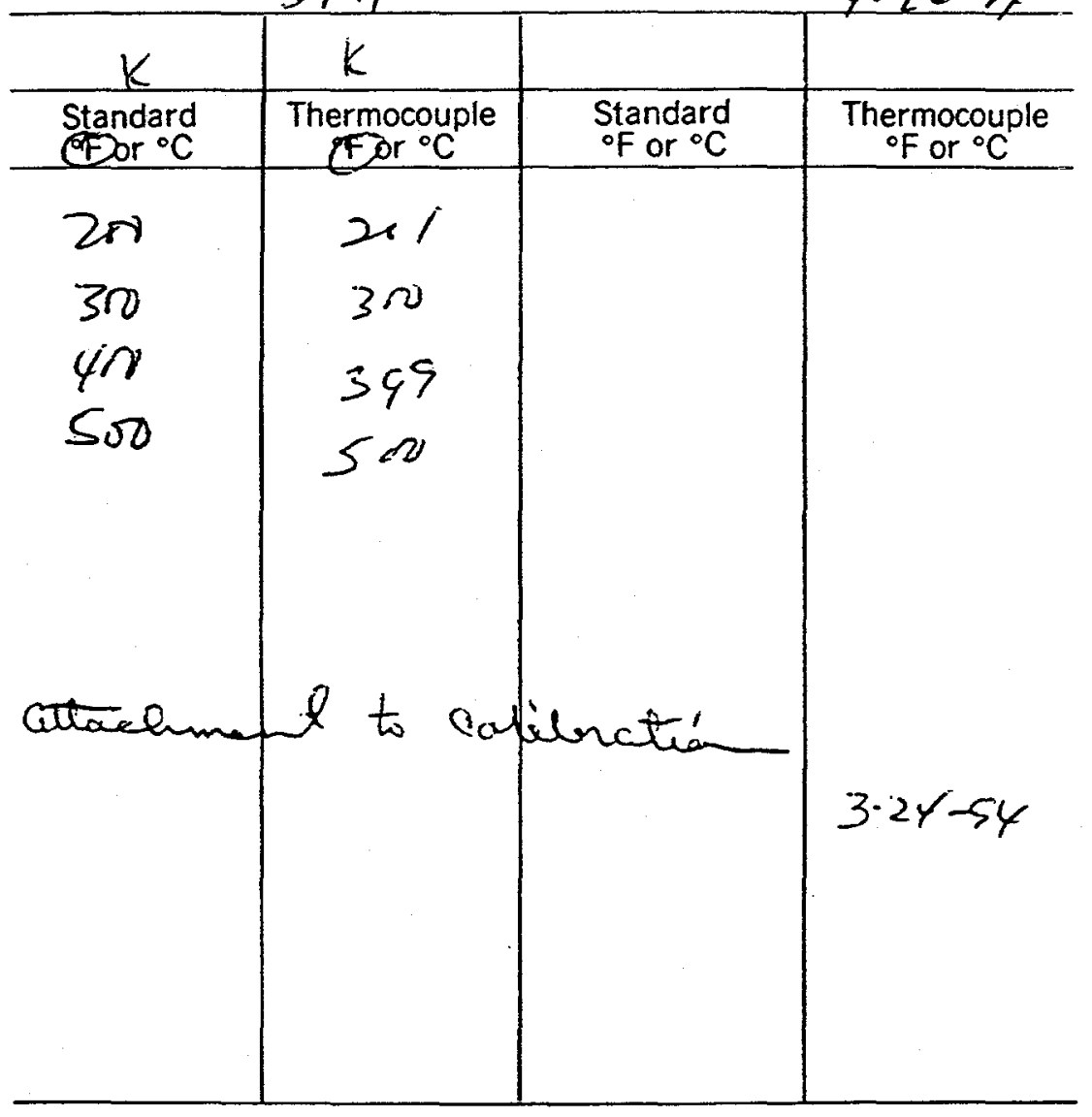

Calibrated by: $C<54$

ECSNo.:

Location:

$1 / 0$

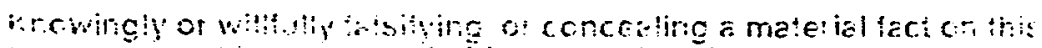

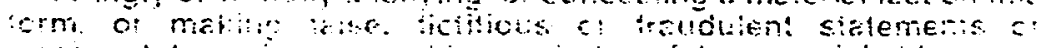

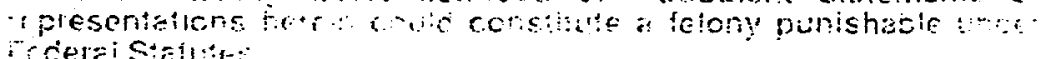

"colet Statute: 
Due Date 3 CALIBRATION OF THERMOCOUPLES

Form Ce

Accuracy \pm 4 of or $\pm 0.75 \%$ of reading (Which ares 1

Procedure No.: NFS-EC-20, Rev. $f$ greater)

NFS I.D. No. SE - 3712

$$
3.25 .84
$$

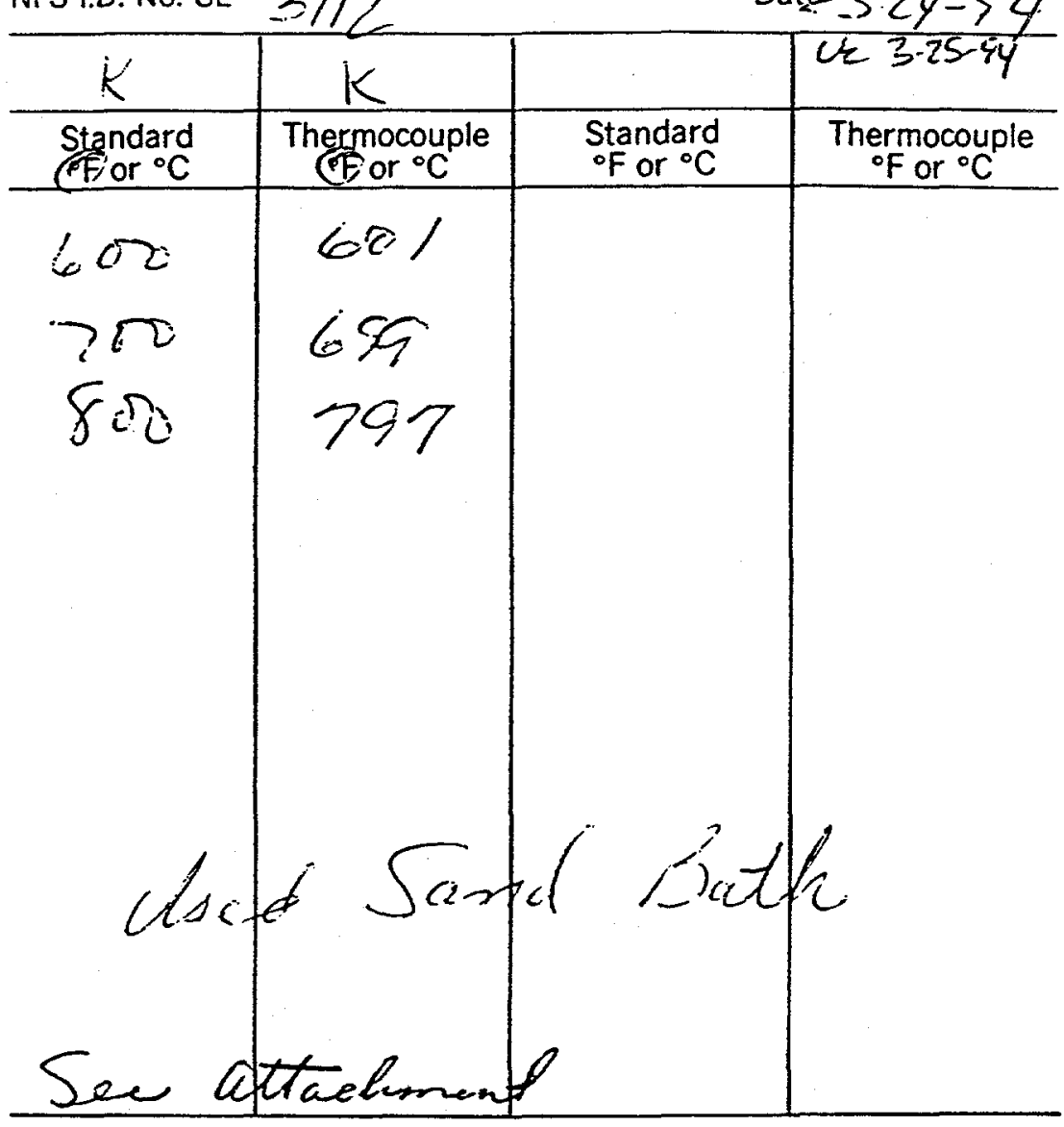

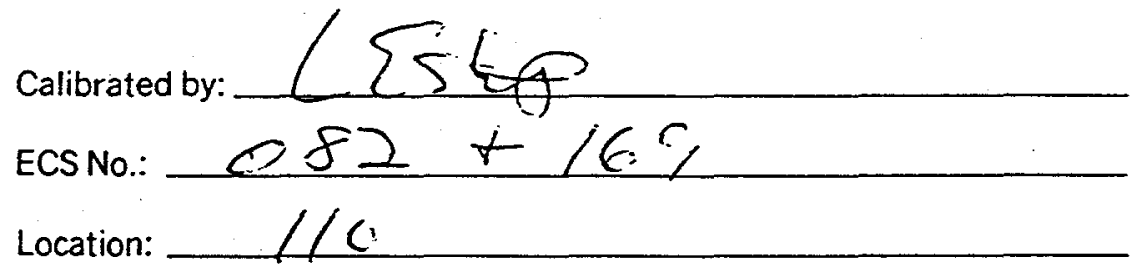

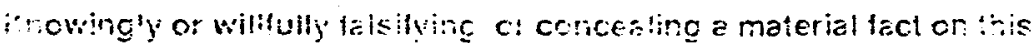

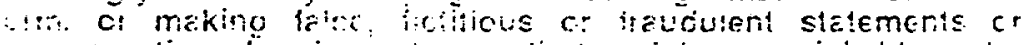

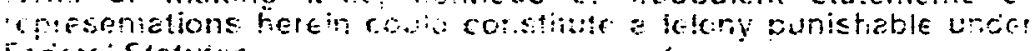
etuis Statutes 
- $A$ CALIBRATION OF THERMOCOUPLES

Form $\mathrm{C} .5$

Due Date $4-26-55$

Accuracy \pm 4 cF

Procedure No.: NFS-EC-20, Rev.

NFS I.D. No. SE - $37 / 2$ Date $4 / 2654$

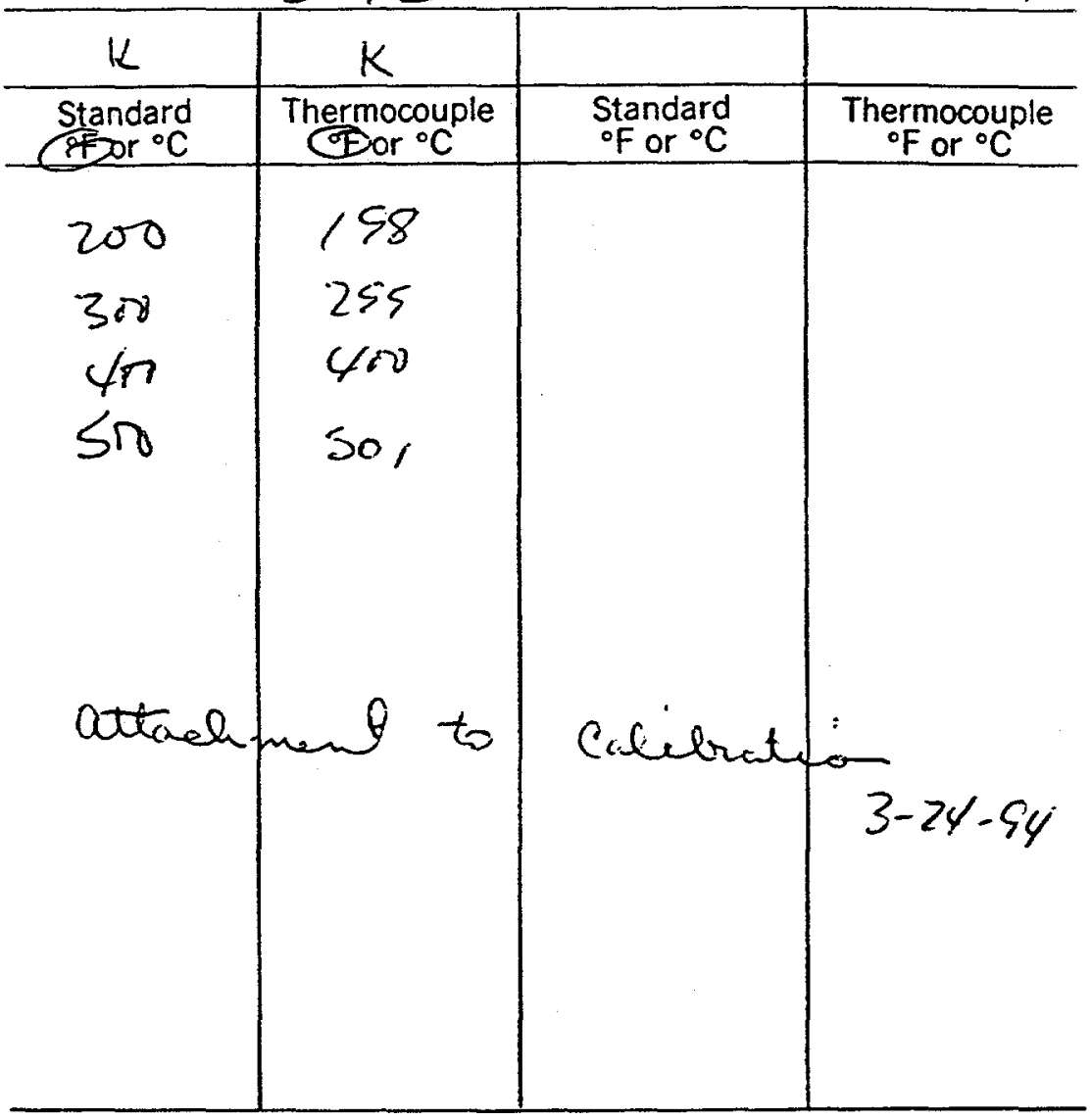

Calibrated by: LESt

ECSNo: $082+169$

Location:

110

Knowingly or wilfully falsifying c! concealing a material fact ch it :s

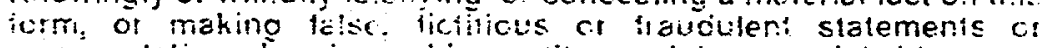

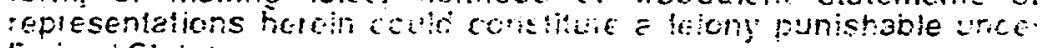
Federal Statutes. 
CALIBRATION OF THERMOCOUPLES

Form C.5

Due Date $1-20-95$

Accuracy $\pm 20.4274^{\circ} \%$

Procedure No.: NFS-EC-20, Rev. 4

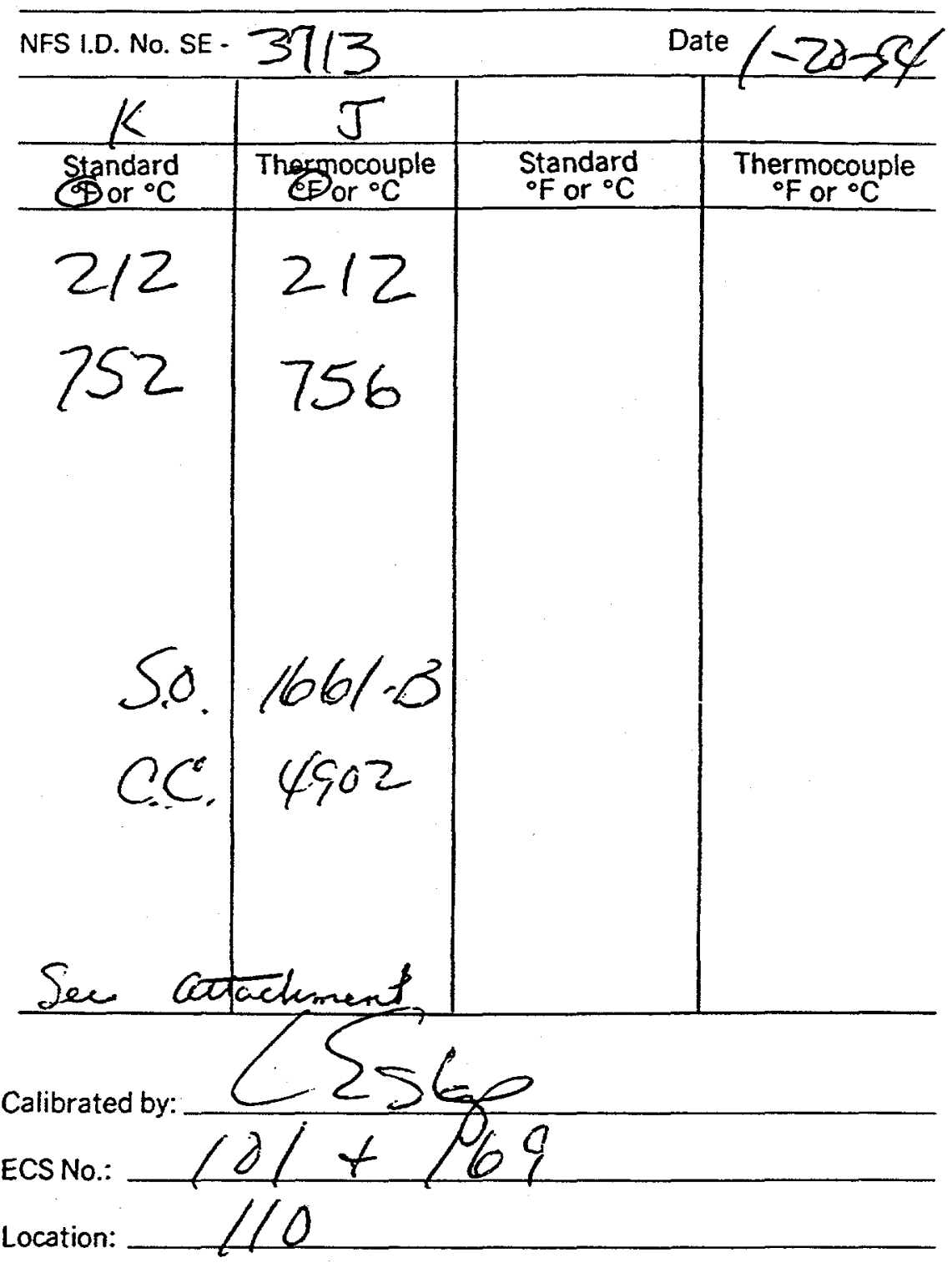

knowingly or will ul waiting or conceding a material fact on this

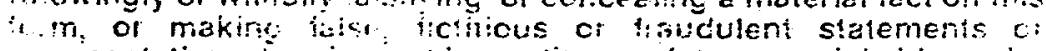

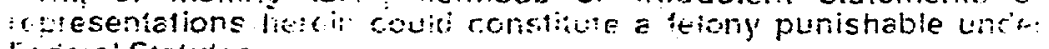
rifelal Srofues. 
- calibration of thermocouples

Form C.5

Due Date 4.27 .95

Accuracy \pm 4 CF

Procedure No.: NFS-EC-20, Rev. 4

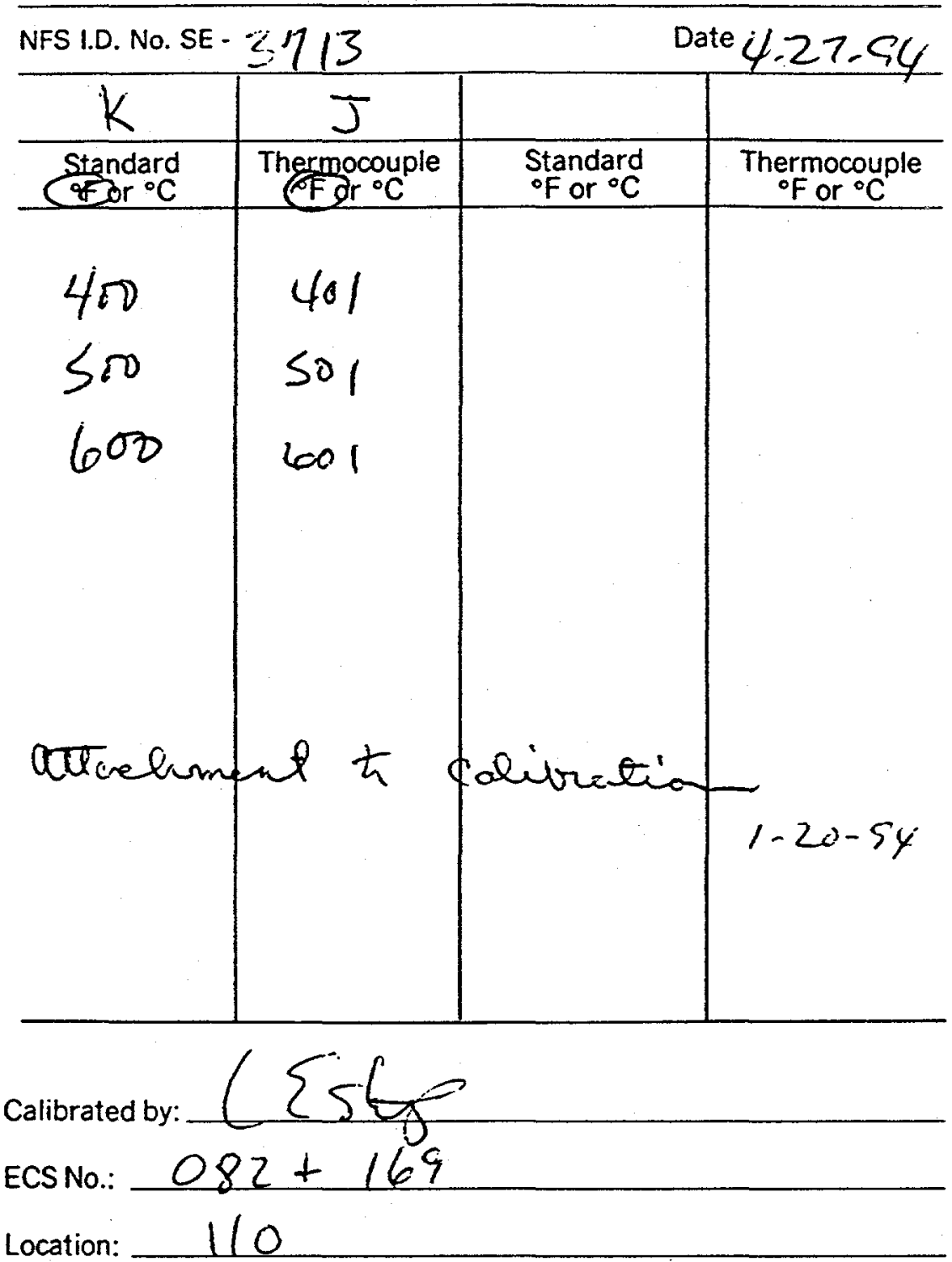

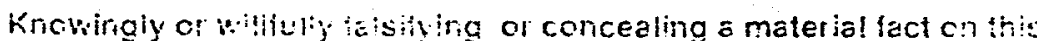

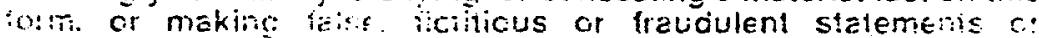

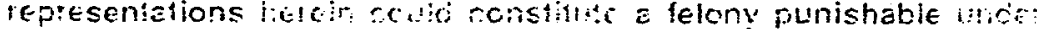

Fecieral Siatutes 
Due Date $4-27-94$

Accuracy $\pm 4 . F$

Procedure No.: NFS-EC-20, Rev. 4

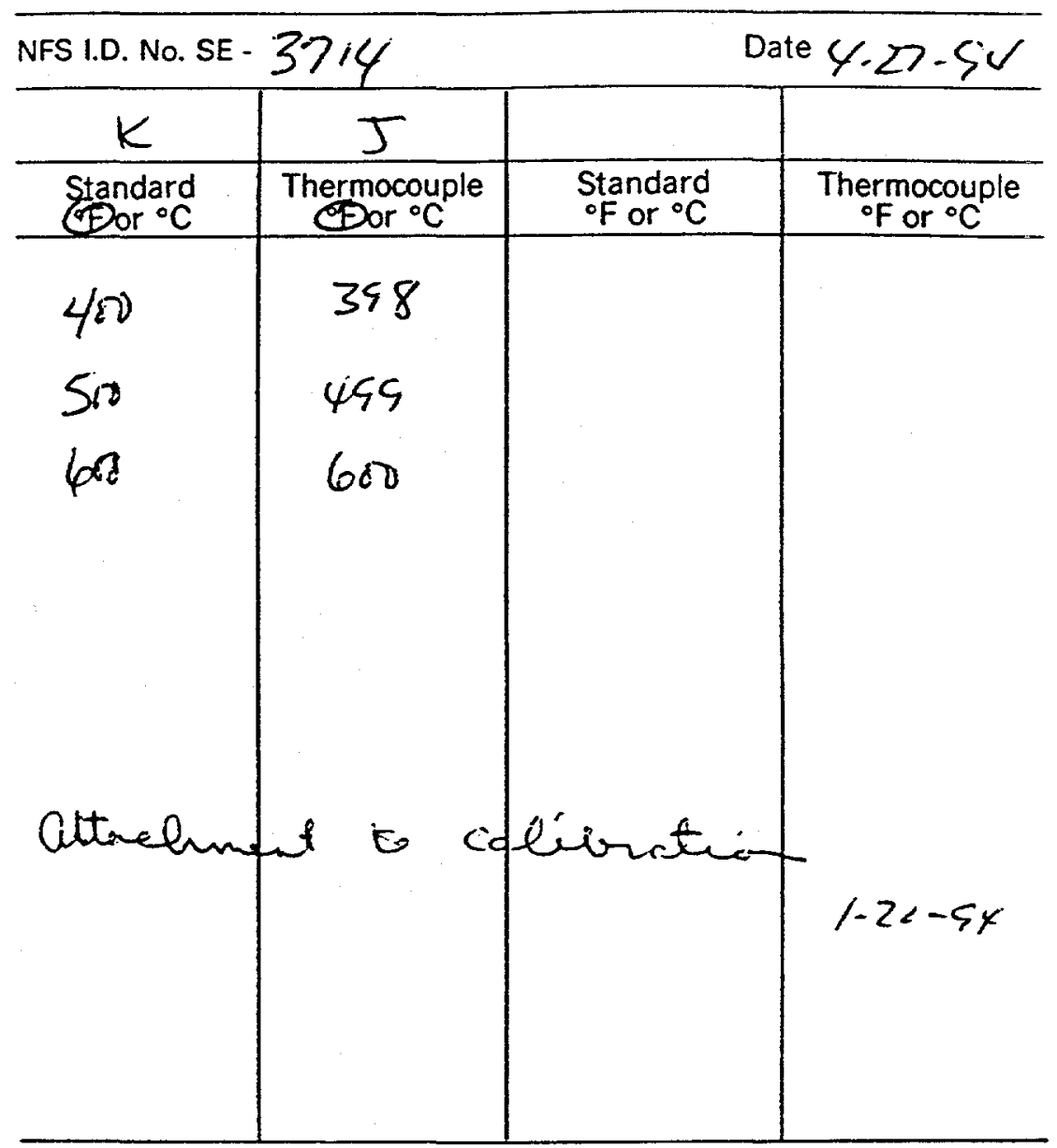

Calibrated by:

$\angle \sum 56$

ECSNo:;

$082+169$

Location:

110

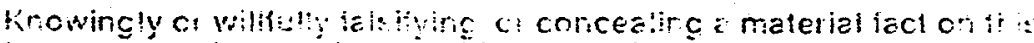

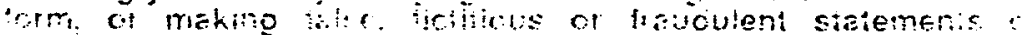

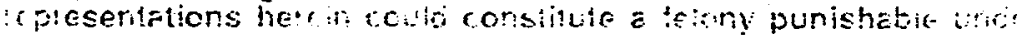

pereral Statules. 


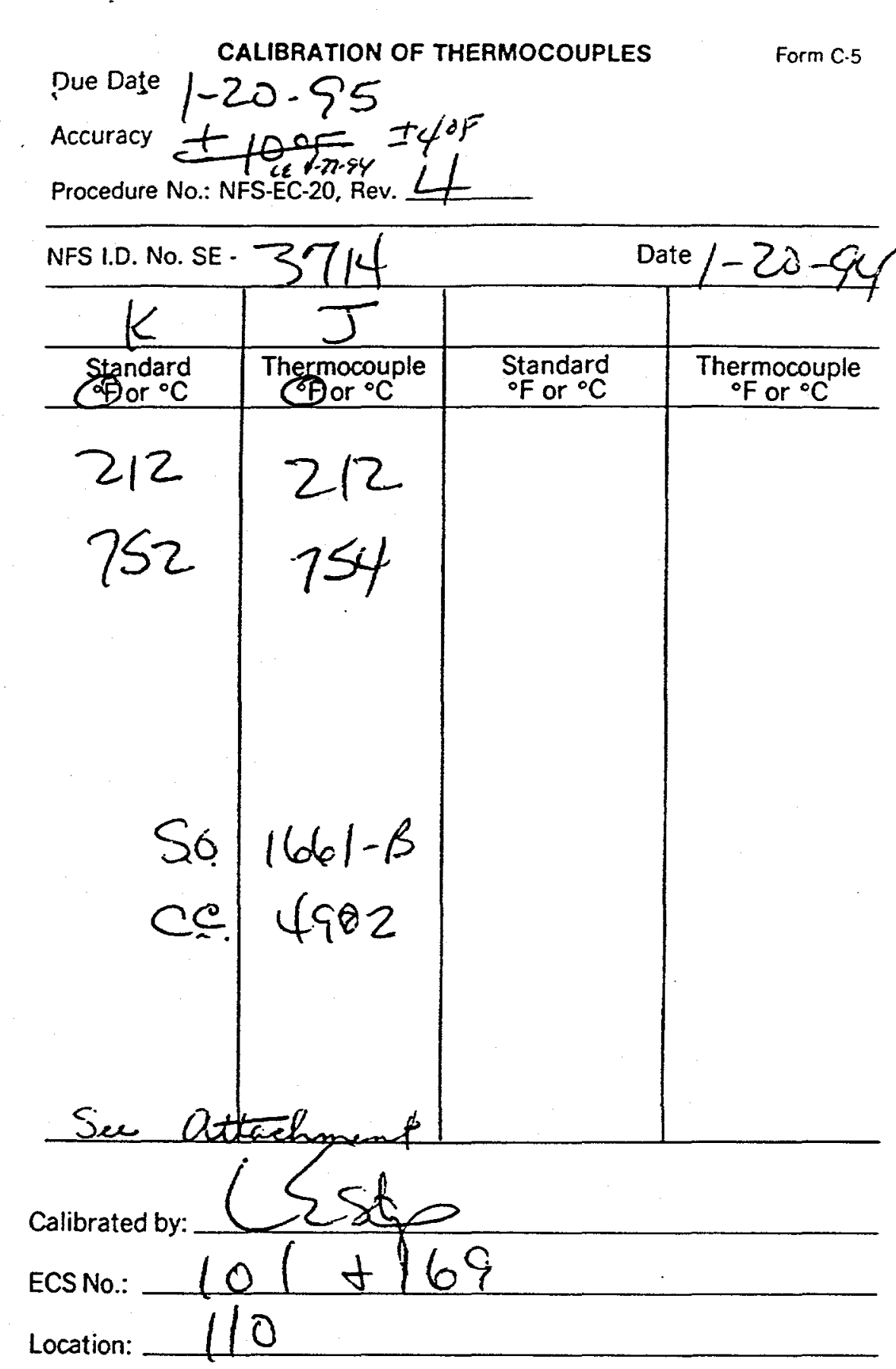

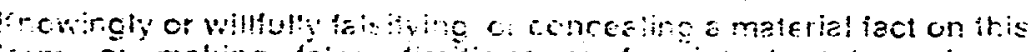

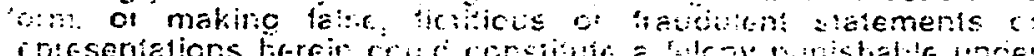
peotral Siatules. 
CALIBRATION OF THERMOCOUPLES

Form C.5

Due Date $4-14-95$

Accuracy $\pm 40 \mathrm{~F}$

Procedure No:: NFS-EC-20, Rev.

NFS I.D. No. SE- $3716 \quad$ Date $4-14-S 4$

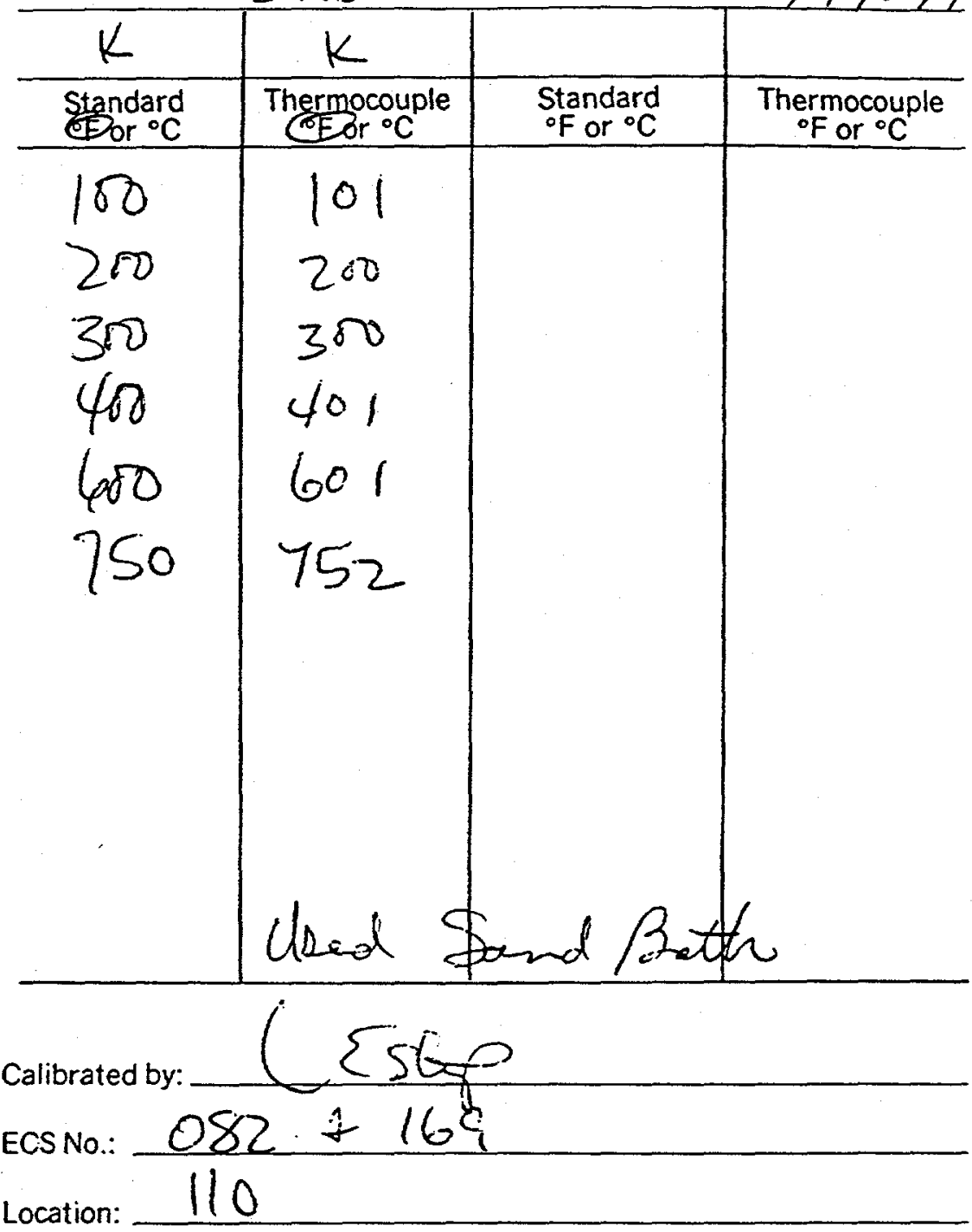

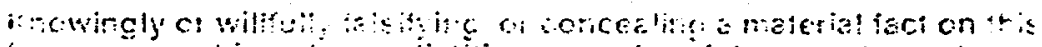

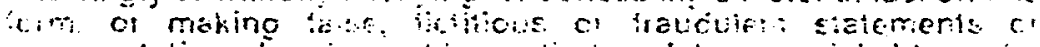

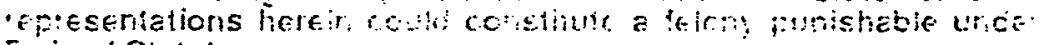
Federal Statutes. 
Due Date 4.1495

Accuracy \pm YoF

Procedure No.: NFS-EC-20, Rev. 4

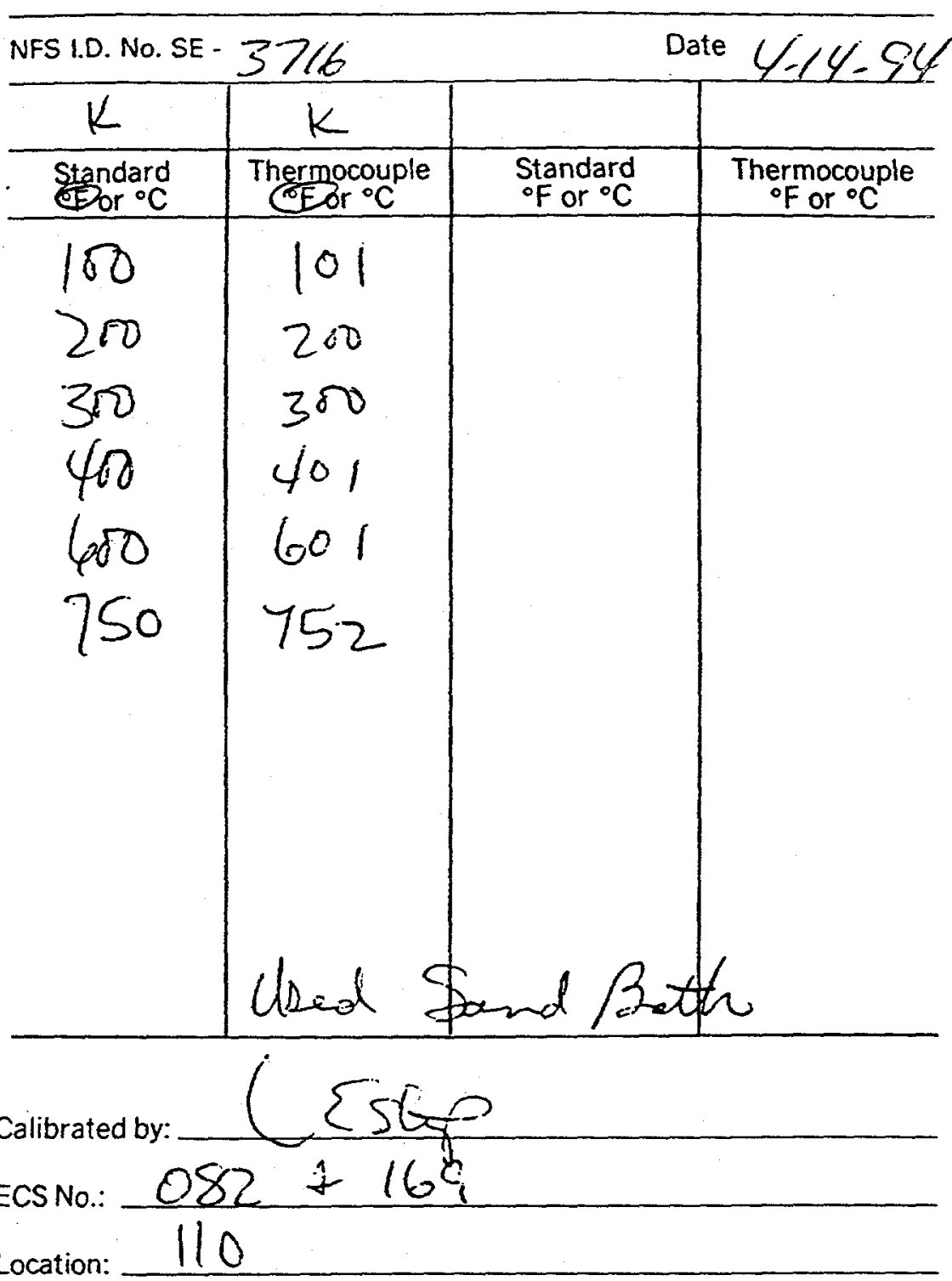

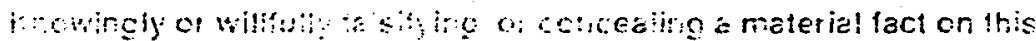

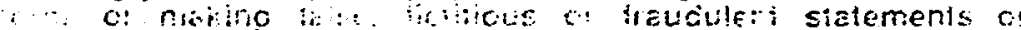

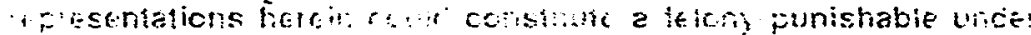

íCEral Siztutes. 
N.F.S. I.D. NO IA -891 Description temp. Fudicifon MODEL NO. None

CALIBRATION FREQUENCY Communally
MANUFACTURER AshCRO

SERIAL No. None

CALIBRATION PROCEDURE No. EC. 10

ACCURACY \pm 10 OF (Readability)

RANGE $200-10000 \mathrm{~F}$

DATE

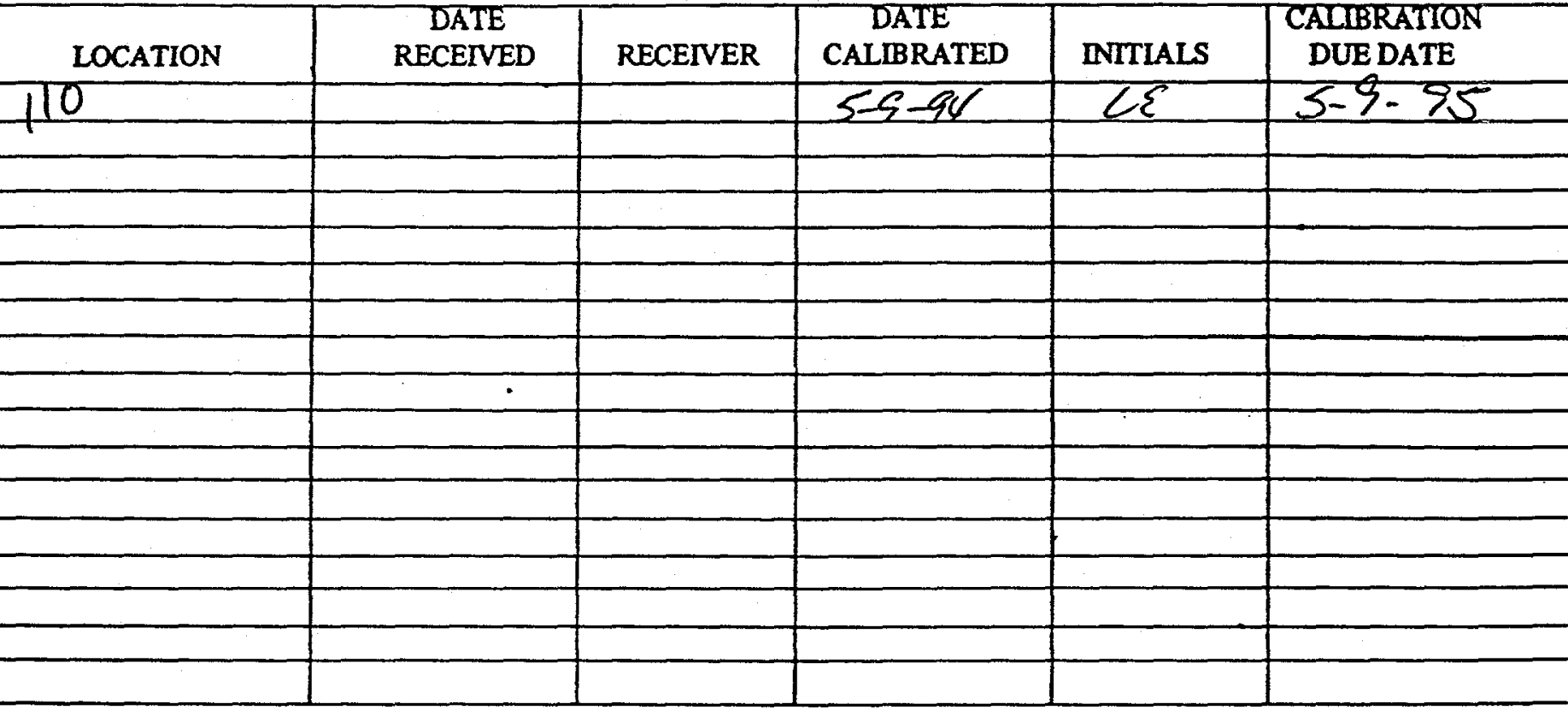


CALIBRATION OF TEMPERATURE INDICATORS

Form C-4

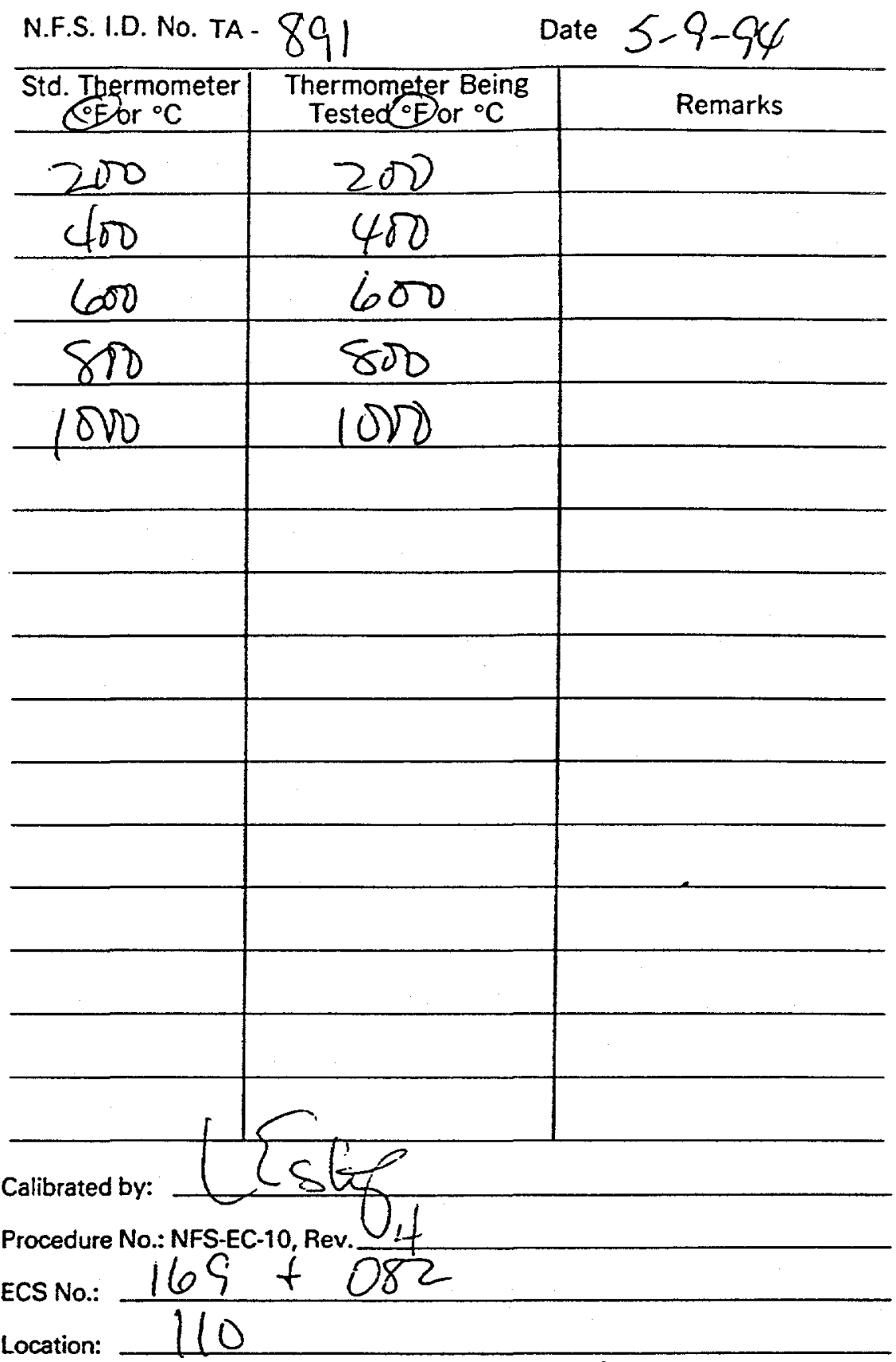

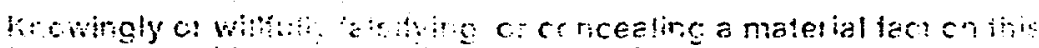

or hisking

iavduten slaichet:s

fretal Siavites 
N.F.S. - ID. NO. MANUFACTURER 7 the 14

description Temp. Controller SERAI NO. $215 / 84$ MODEL No. $4000 \mathrm{~F}$ CALIBRATION PROCEDURE NO. $\& \mathrm{C} \cdot 12$

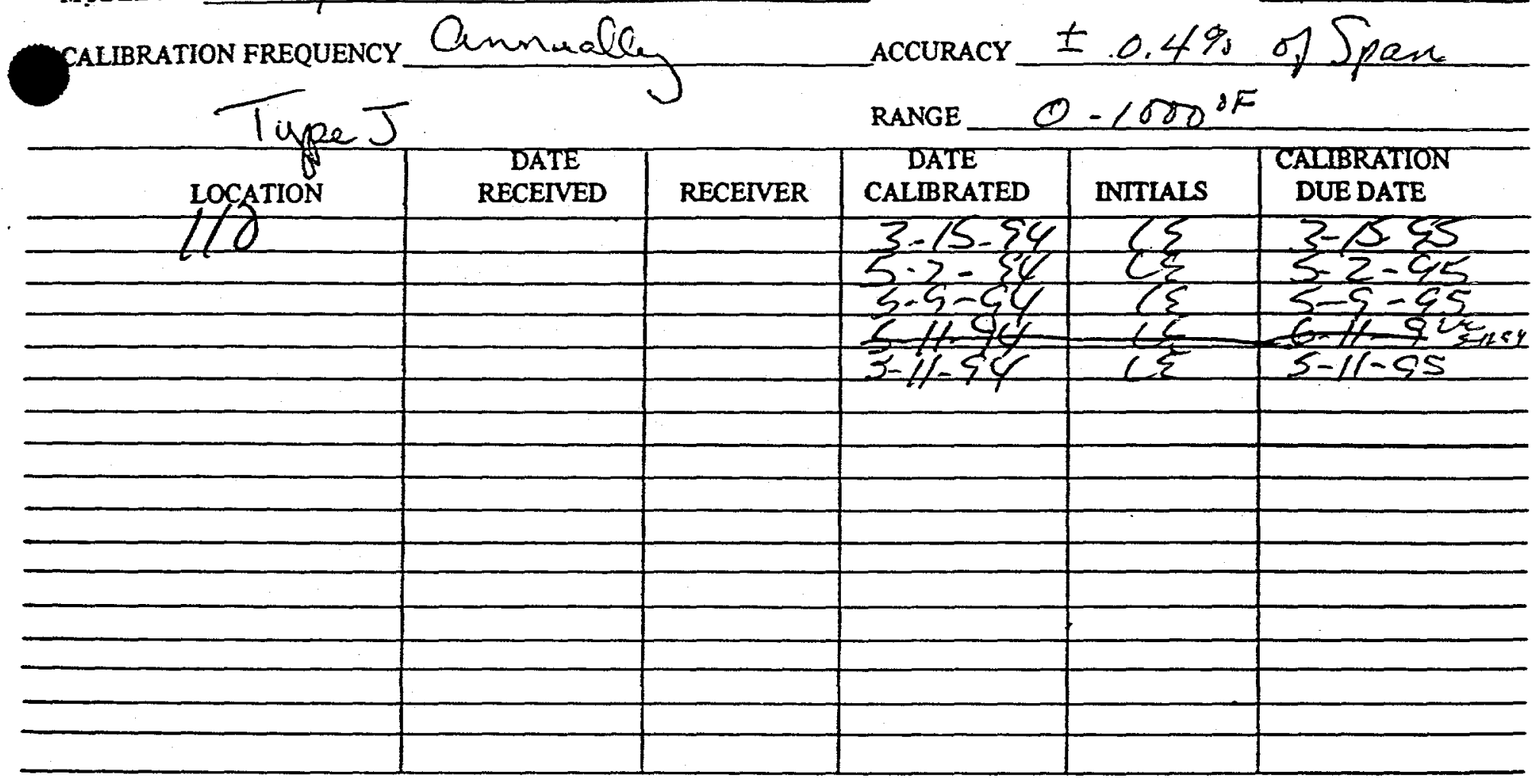



AND RECORDERS

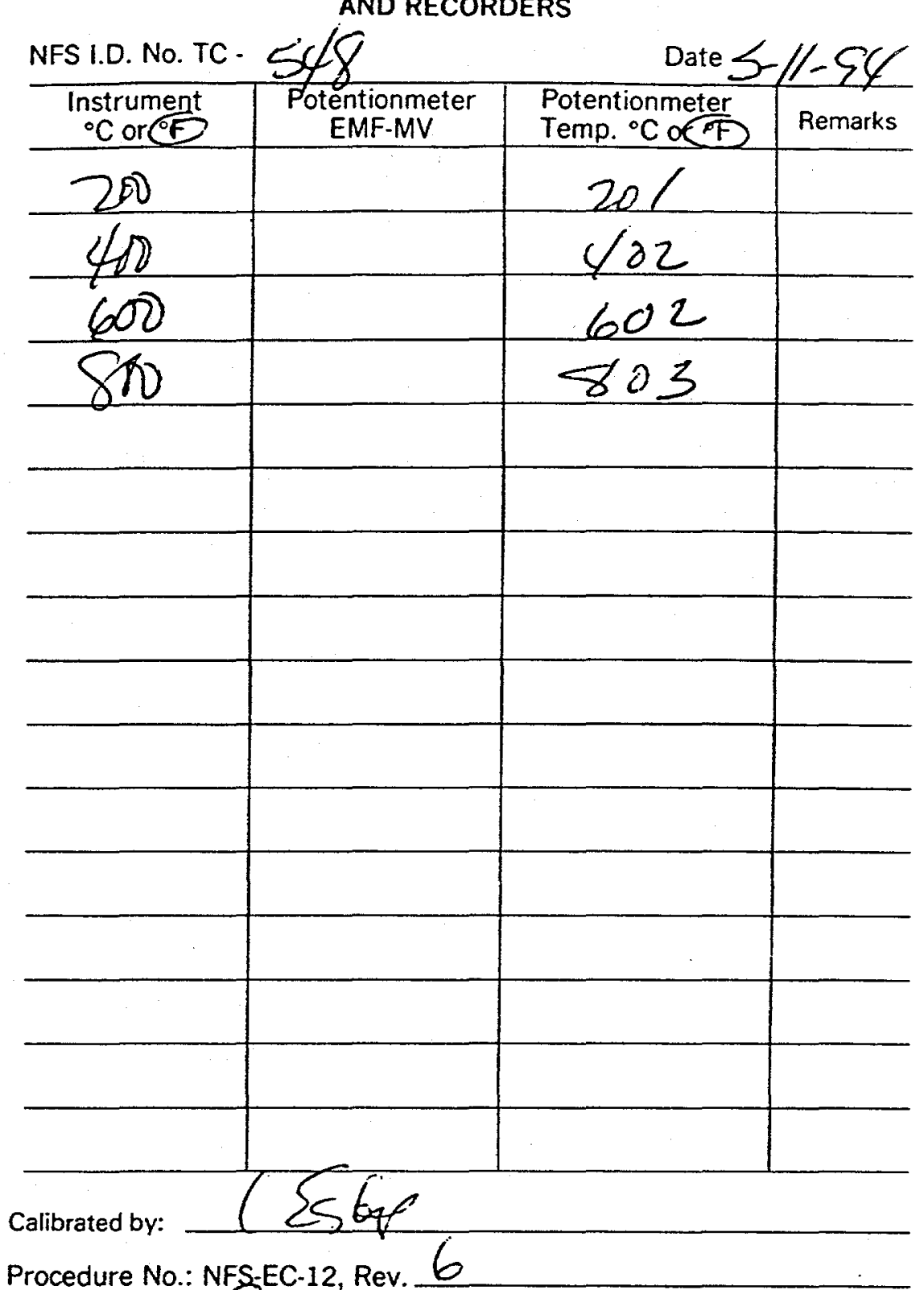

ECS No:

Location:

Knowingly or willfully falsifying or concealing a material fect on this

form, or making false, fictitious or fraudulent statements of

representations herein cculd consiitule a felony punishable unot

Federal Statutes. 


\section{AND RECORDERS}

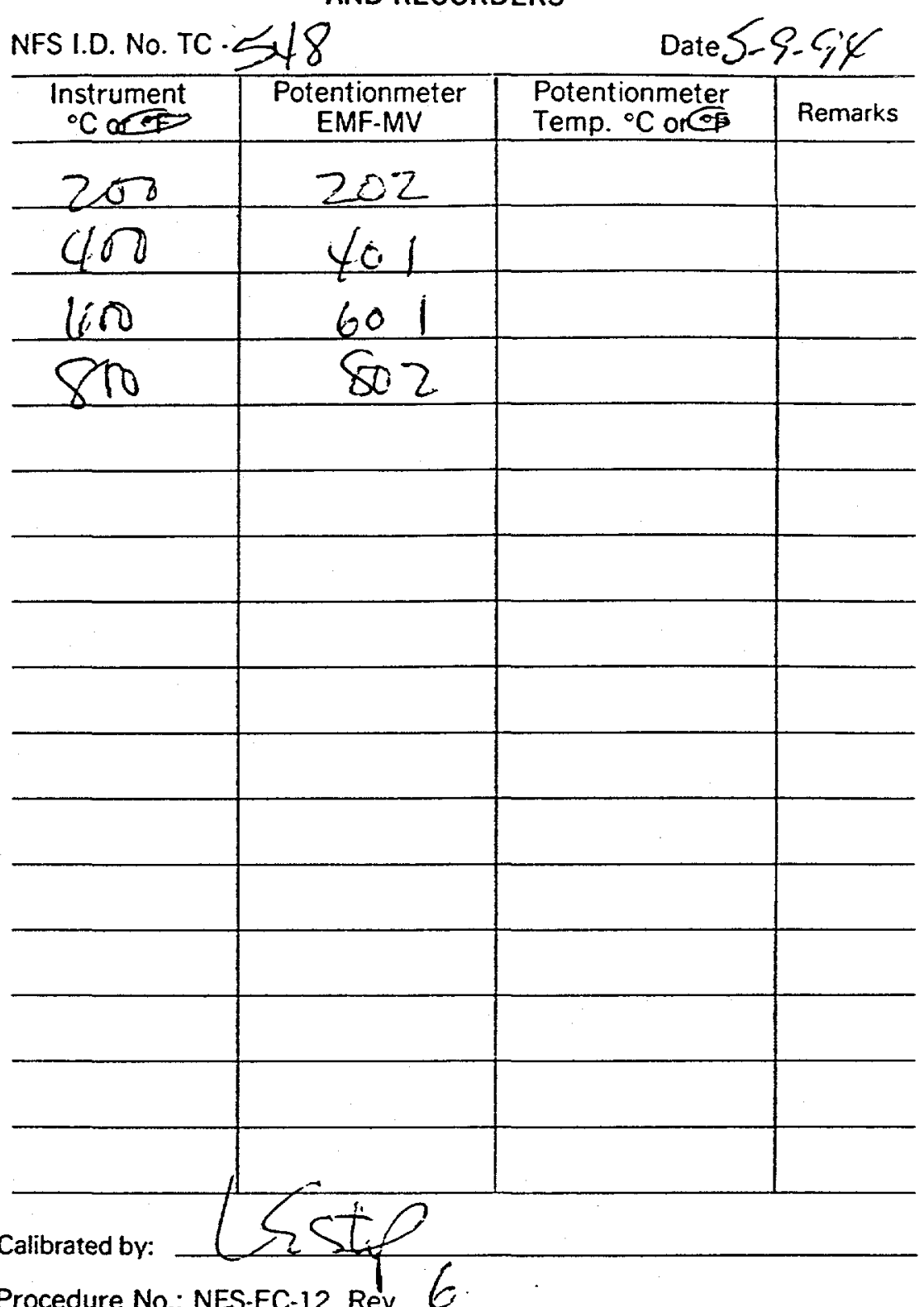

Procedure No.: NES-EC-12, Rev. $G$

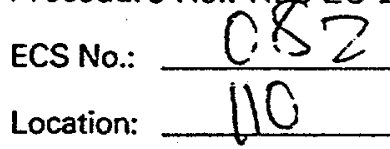

Knowingly or willfully talsifyine or concealing a material fact on this form, or making feise. fictitious of traudulent statements of representations herein rovic constituie a ieiony punishable unde: Federal Statutes. 
CALIBRATION OF TEMPERATURE CONTROLLERS FOrM C.1 AND RECORDERS

NFS I.D. No. TC - $54 / 8 \quad$ Date $5.2-84$

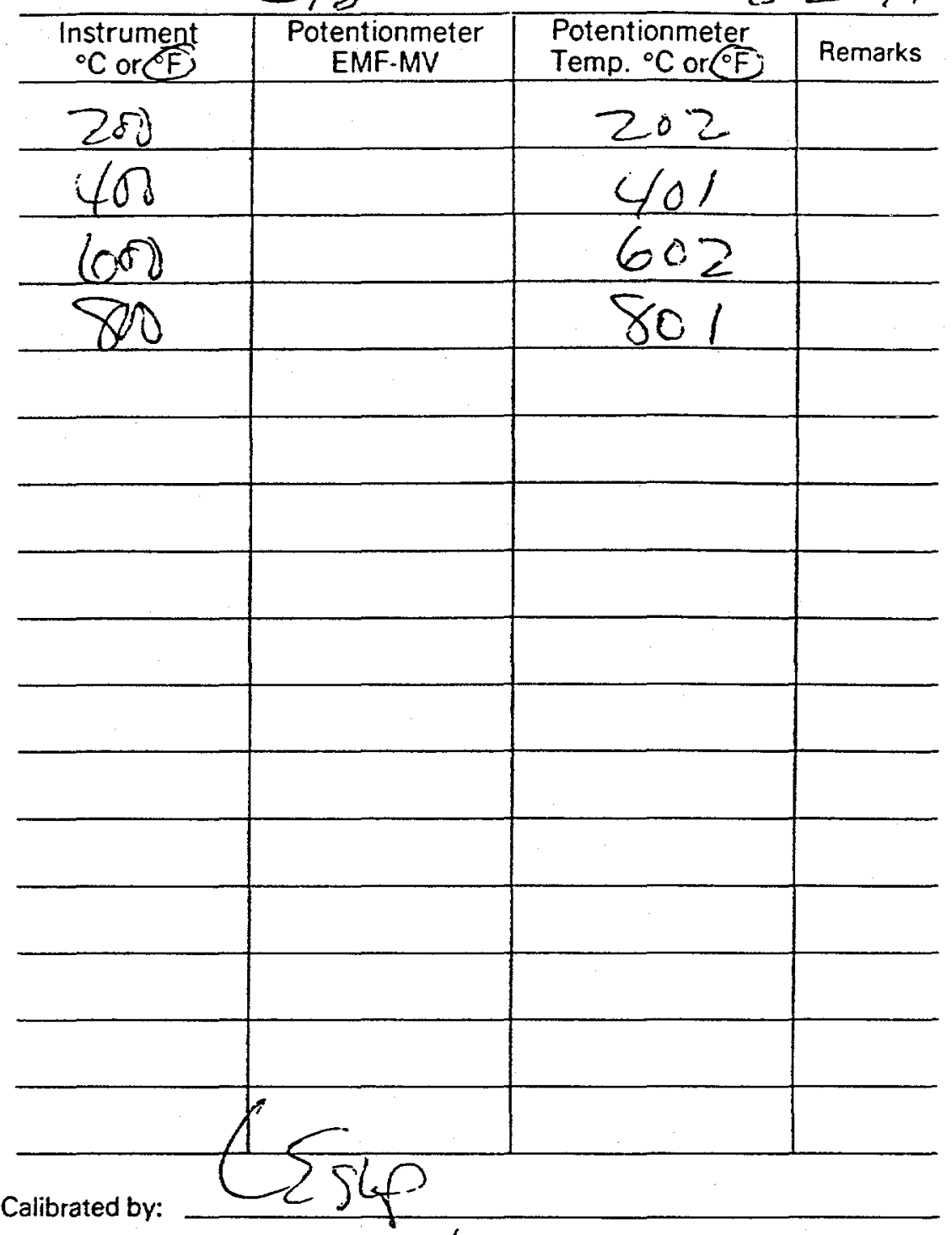

Procedure No.:NFS-EC-12, Rev. 6

ECSNo.: $\mathrm{C}, \mathrm{S}$

Location: $\mathrm{H}(\mathrm{C}$

Knowingly or willfully falsifying or concealing a material fact $\mathrm{cr}$, this form, or making false, fictitious or fraudulent statements $c$ : Federal Sialuites. 
CALIBRATION OF TEMPERATURE CONTROLLERS FOrm C.1 AND RECORDERS

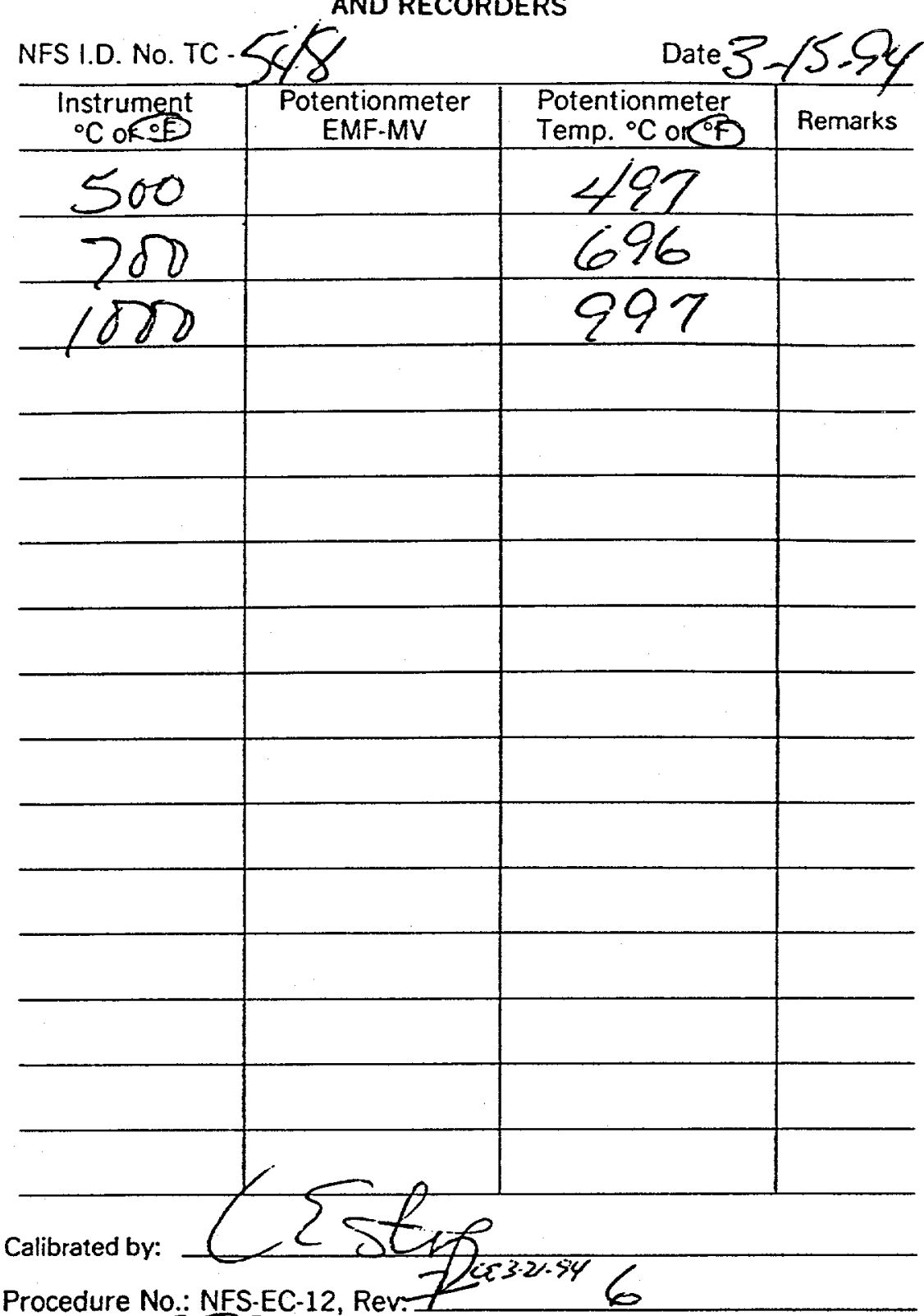

Procedure No.: NFS-EC-12, Rev:-

ECS No.:

Location:

$$
882
$$

Knowingly or willfulty falsifying or concealing a material fact on this form, or making false, fictitious or fraudulent statements or representations herein could constitule a felony punishabie undc: Federal Statules. 
N.F.S.- I.D. NO.TC . S50

Descrprion temp Recender.

MODEE No. CT 7100

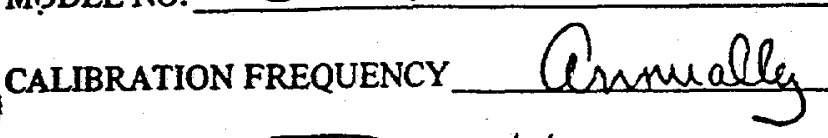

MANUFACTURER OM ega SERAL No. $93 k 74524$ CALLBRATION PROCEDURE NO. \&C. 12

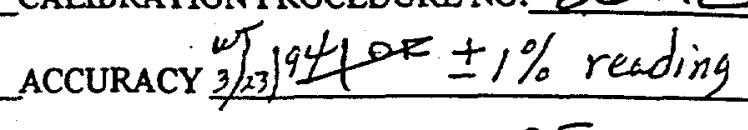

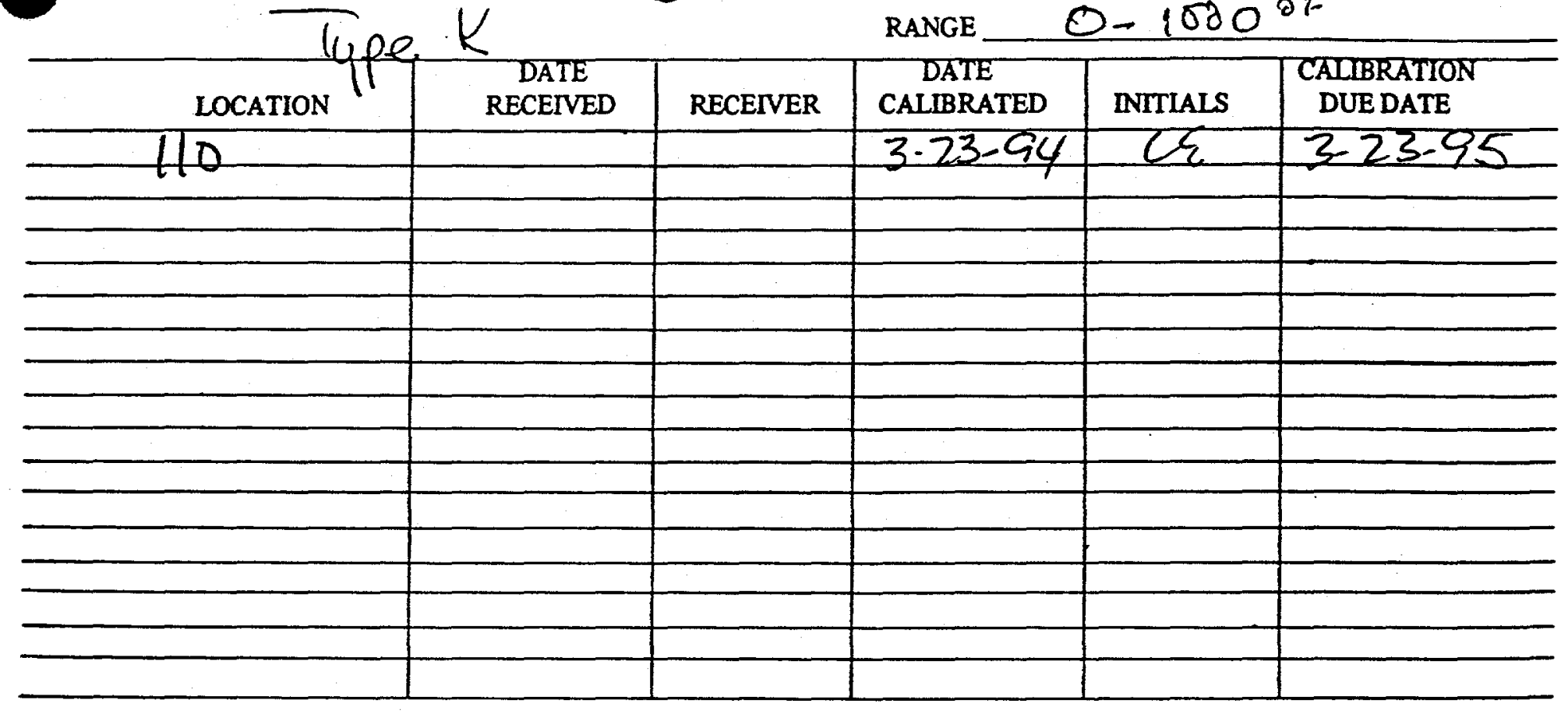


$\cdot \quad \because$ CALIBRATION OF TEMPERATURE CONTROLLERS Form C. 1 AND RECORDERS

NFS I.D. No. TC - $550 \quad$ Date 3.23 .44

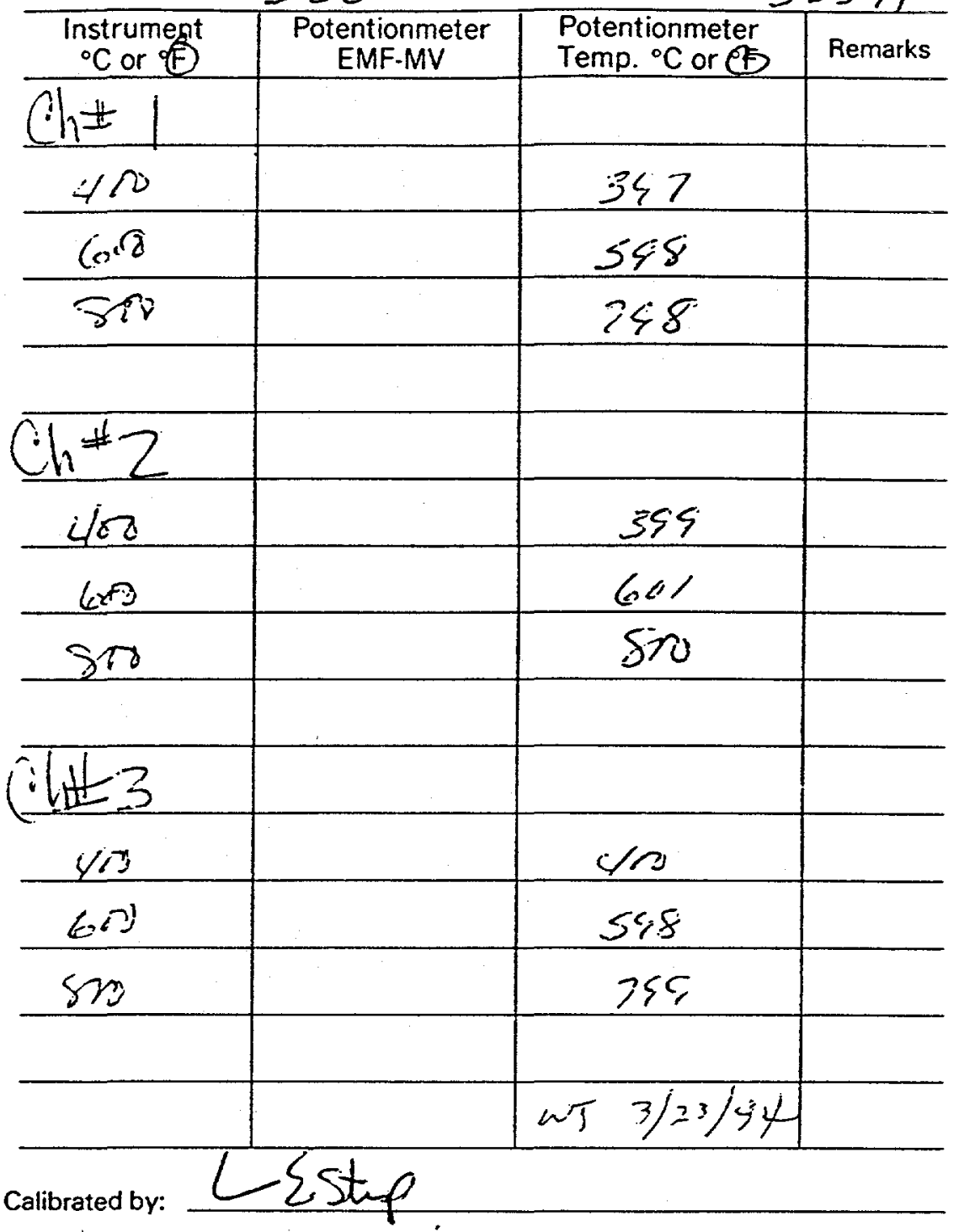

Procedure No.: NFS-EC-12, Rev. 6

ECSNo.: 082

Location:

Knowingly or willfully falsifying or concealing a material fact on this form, or making false, fictitious or fraudulent statements $\mathrm{cr}$ representations herein could constitute a felony punishable under Federal Statutes. 
$\because \quad \because$ CALIBRATION OF TEMPERATURE CONTROLLERS Form C-1 AND RECORDERS

NFS I.D. No. TC - $550 \quad$ Date 3-23-94

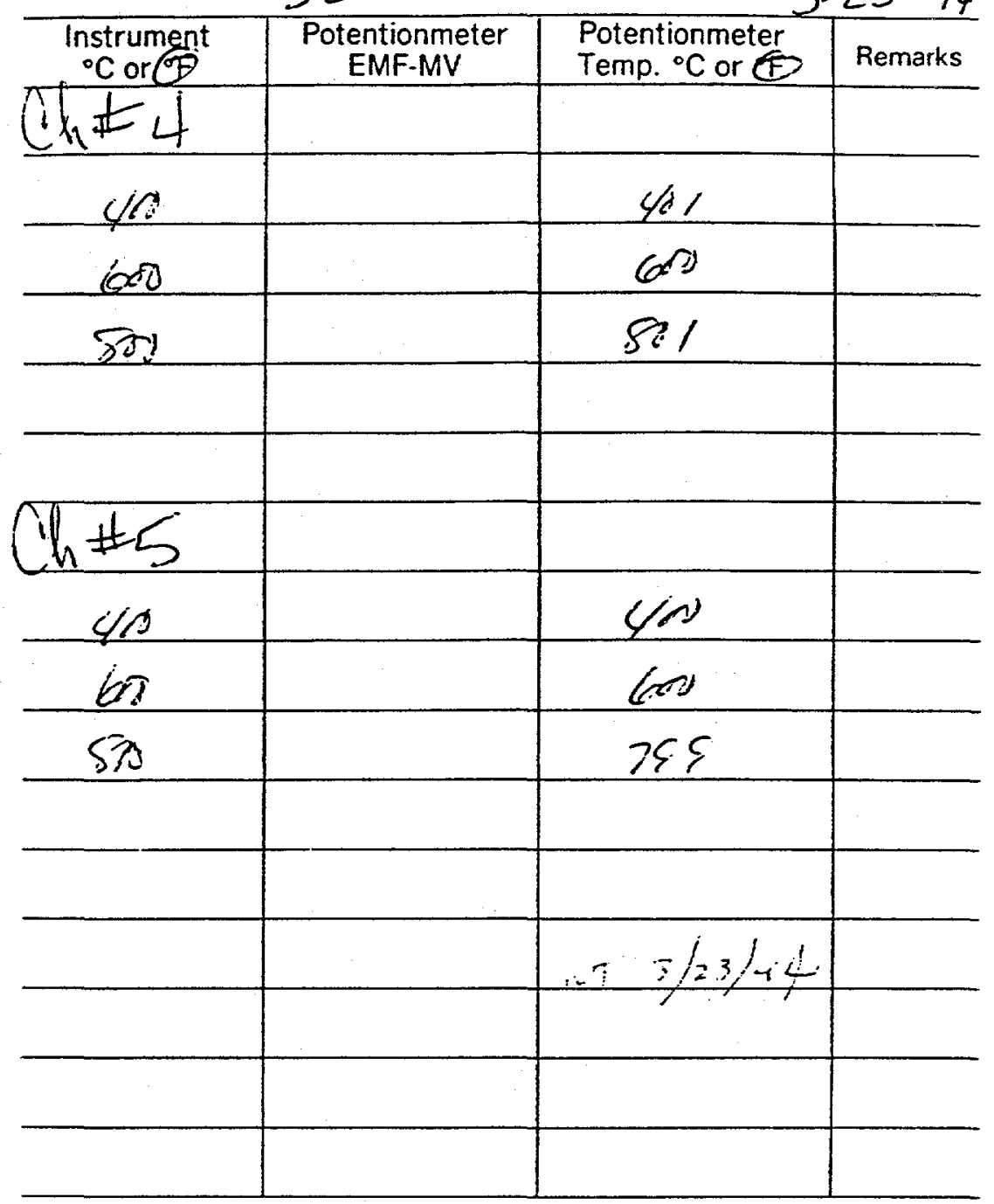

Calibrated by: CaStro

Procedure No.: NFS-EC-12, Rev.

ECS No.: $\frac{082}{110}$

Knowingly or wilfully falsifying or concealing a material fact on this form, or making false, fictitious or fraudulent statements o representations fietein could constitute a felony punishable lion: Federal Statutes. 


\begin{tabular}{|c|c|c|c|c|c|}
\hline \multicolumn{3}{|c|}{$\begin{array}{l}\text { N.F. ID NO. WC. } 051 \\
\text { DESCRPTION } \frac{\text { Dh }}{W \text { form }} \text { Schle } \\
\text { MODEL No. } W 1-110\end{array}$} & \multicolumn{3}{|c|}{ 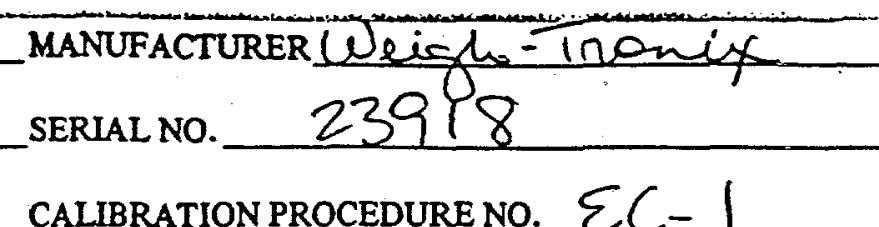 } \\
\hline & & & RANGE $\mathrm{O}$ & -100 & $16 s$ \\
\hline LOCATION & $\begin{array}{c}\text { DATE } \\
\text { RECENED }\end{array}$ & RECENVR & $\begin{array}{c}\text { DATE } \\
\text { CALBRRATED }\end{array}$ & INTTALS & $\begin{array}{l}\text { CALIBRATION } \\
\text { DUE DATE }\end{array}$ \\
\hline 110 & & & $\frac{3.22 .94}{4-15-54}$ & रह & $\frac{4-22-94}{5-19-54}$ \\
\hline & & & 年-2-94 & $\{\xi$ & $\frac{6-2-54}{6-5-94}$ \\
\hline & & & & & \\
\hline & & & & & \\
\hline & & & & & \\
\hline & & & & & \\
\hline & & & & & \\
\hline & & & & & \\
\hline & & & & & \\
\hline
\end{tabular}


CALIBRATION OF SCALES AND BALANCES

Form C-3

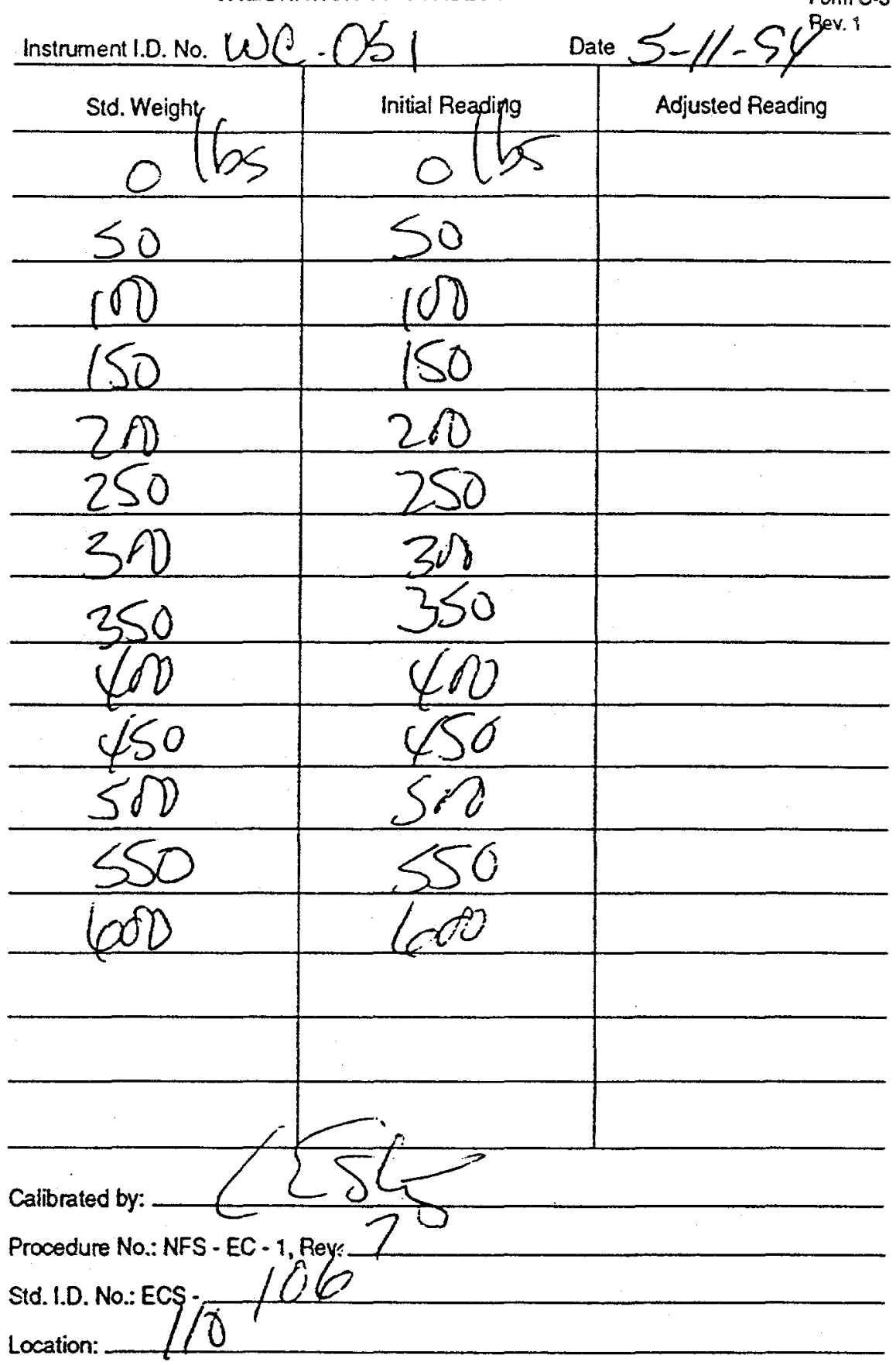

Location:

Knowingly or willfully falsifying or concealing a material fact on this form, or making false, fictitious or fraudulent statements or representations herein could constitute felony punishable under Federal Statutes. 


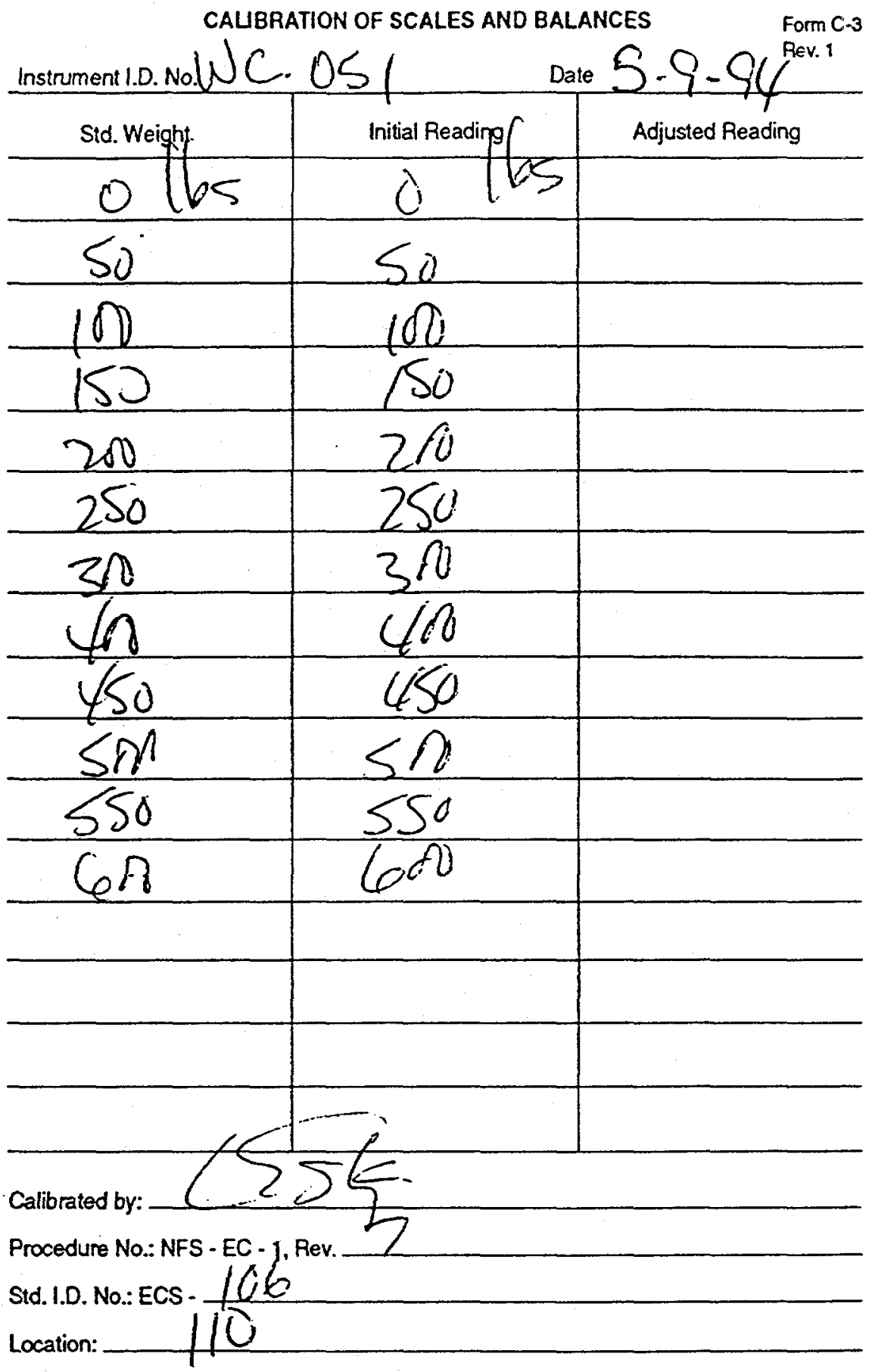

Knowingly or willfully falsifying or concealing a material fact on this form, or making false, fictitious or fraudulent statements or representations herein could constitute felony punishable under Federal Statutes. 


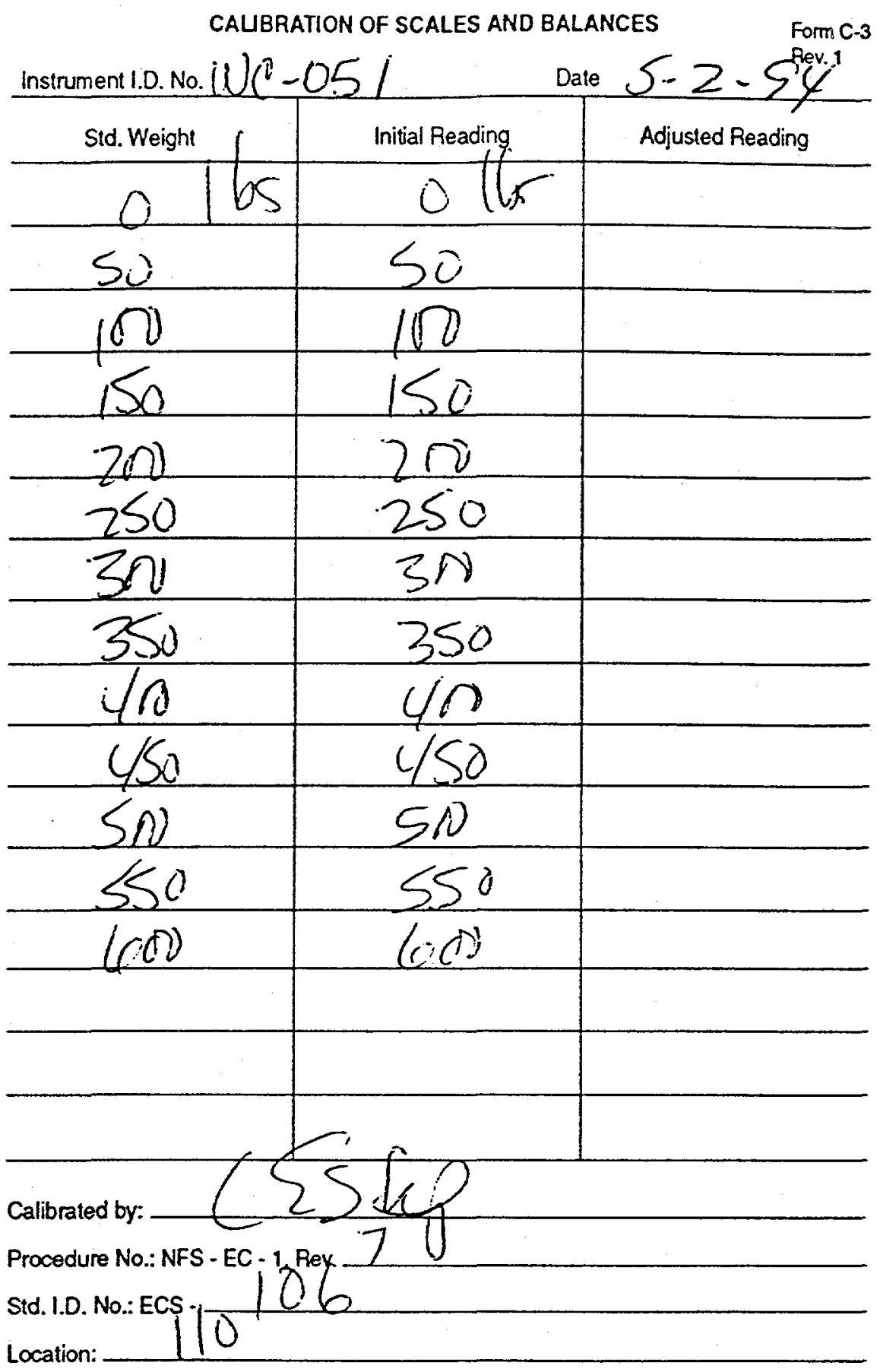

Knowingly or willfully falsifying or concealing a material fact on this form, or making false, fictitious or fraudulent statements or representations herein could constitute a felony punishable under Federal Statutes. 
CAUBRATION OF SCALES AND BALANCES

Form $\mathrm{C} 3$

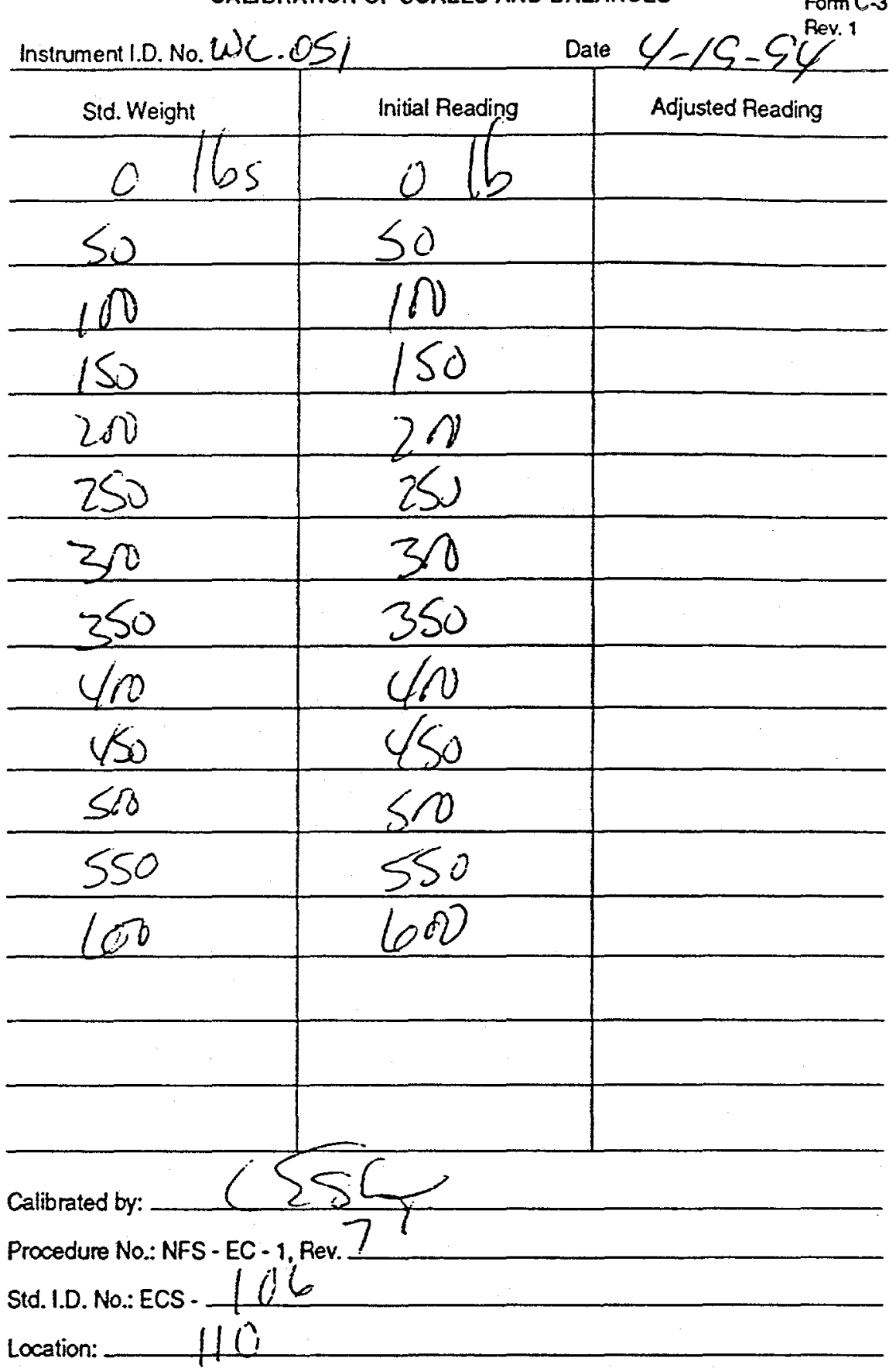

Knowingly or willfully falsifying or concealing a material fact on this form, or making false, fictitious or fraudulent statements or representations herein could constitute felony punishable under Federal Statutes. 


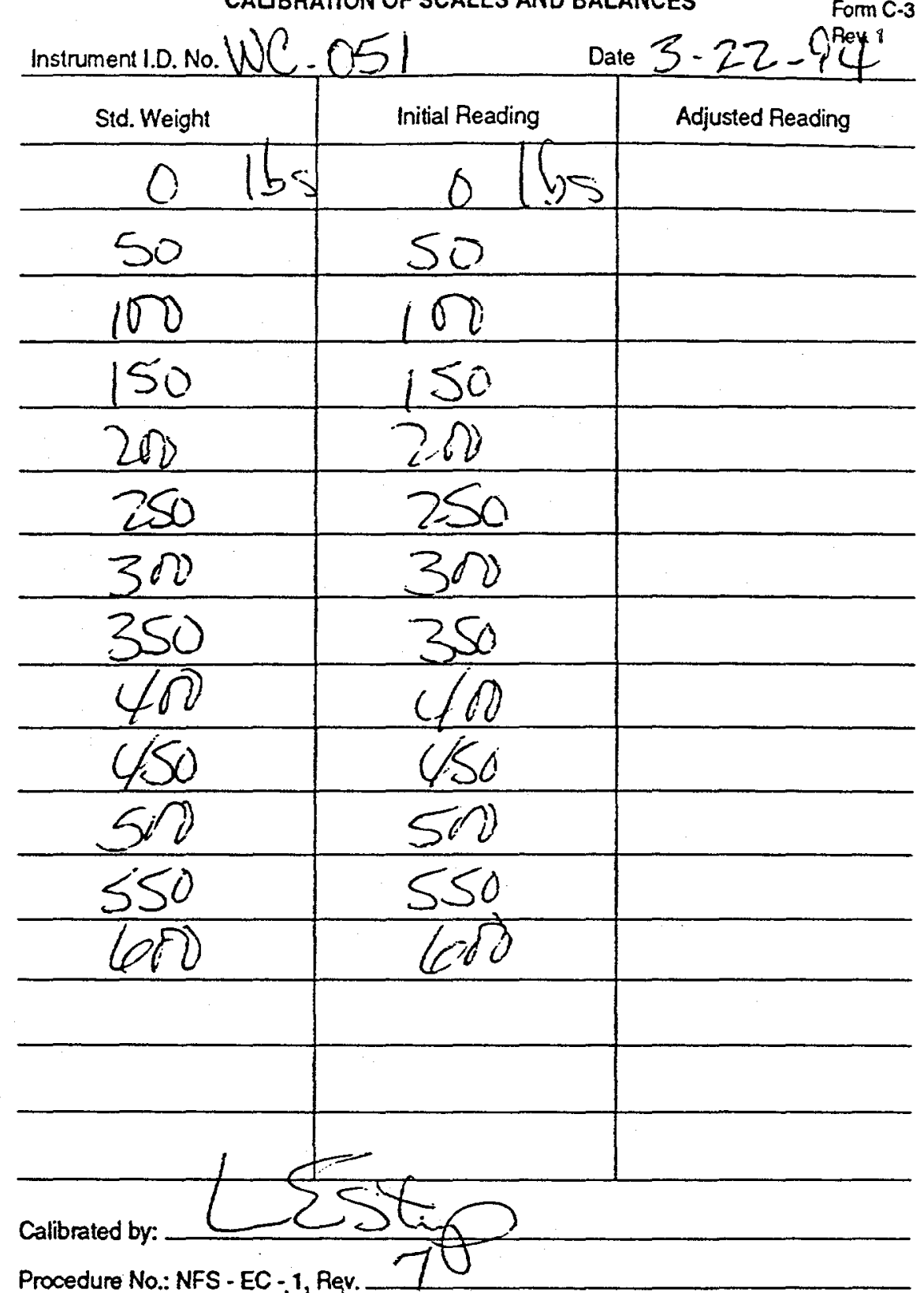

Procedure No.: NFS - EC - 1, Rev.

Std. I.D. No: ECS -

Location:

WT $3 / 22 / 44$

Knowingly or willfully falsifying or concealing a material fact on this form, or making false, fictitious or fraudulent statements or representations herein could constitute a felony punishable under Federal Statutes. 


\title{
APPENDIX II
}

\section{QUALITY ASSURANCE}

\author{
QA Assessment March 24, 1994 \\ QA Assessment LEFPC -94-001 \\ QA Assessment LEFPC -94-002
}

Corrective Action Response May 11, 1994

QA Review of Corrective Action Response May 11, 1994

QA Monthly Report $\begin{array}{r}\# 1 \\ \# 2 \\ \# 3 \\ \# 4\end{array}$

Readiness Review

Training Records

Laboratory Evaluations 


\section{MEMORANDUM}

March 24, 1994
23T-94-0025

QUA-06

CWT-94-005

TO: $\quad$ Robert Burckhardt

FROM: Wade Tilson

SUBJECT: MMES Quality Assurance Assessment Finding

RE:

\section{LEFPC Project}

During a Quality Assurance assessment of the Lower East Fork Poplar Creek Project, now under way at NFS, Mr. Hugh C. Newsom, an MMES Oak Ridge auditor, identified the following items in your area of responsibility:

1) Purchase Order B9403 00318 (REQ 39247) to Roy F. Weston, Inc. did not include the Quality Assurance requirements specified in the requisition, i.e., they were inadvertently left out. Also, in the same P.O., the analysis being requested (Dioxin) was badly misspelled.

2) Purchase Order B9311 00291 (Req 31037) to EcoTek LSI has an error associated with the DQO levels. The purchase requisition stated "(DQO) Levels II or IV", the Purchase Order stated "DQO levels LLL or LV". This error is obviously a typo and may appear to be minor, however, our customer considered this item significant enough for an audit finding.

To immediately resolve the above items, purchase requisitions have been written (29164 to Roy F. Weston, Inc., and 29167 to EcoTek LSI) amending the two Purchase Orders to include the required information. In an effort to prevent similar occurrences on the LEFPC Project, I am requesting that a QA representative review future P.O's prior to issuance. This is not only my request, but was strongly requested by the MMES - Oak Ridge auditor. A QA review of P.O.'s on this project is required by the project QA Plan (NFS-Q-184, Rev. 1, Paragraph 6.0).

Attached for your information is a copy of the audit finding sheet.

Your cooperation in resolving this matter would be appreciated.

ckf

attachment

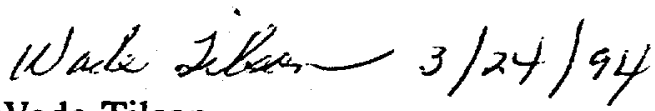

Wade Tilson 
FINDING/OBSERVATION REPORT

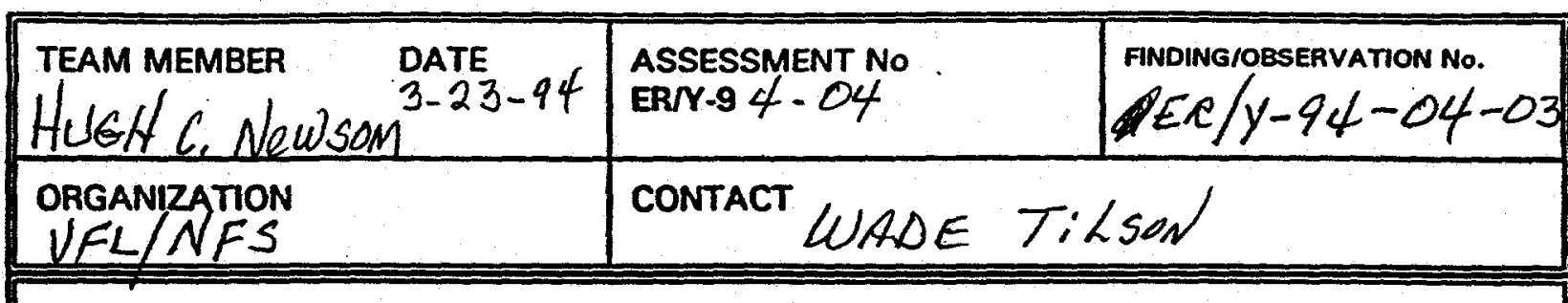

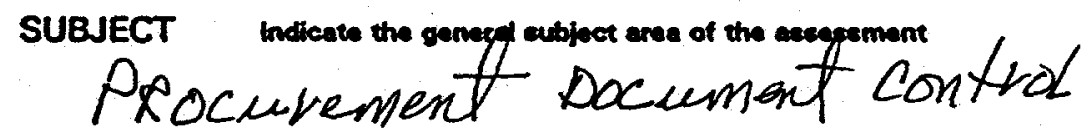
REQUIREMENT(S)

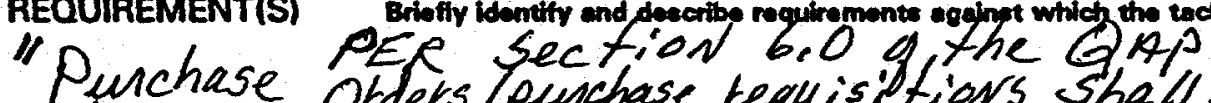

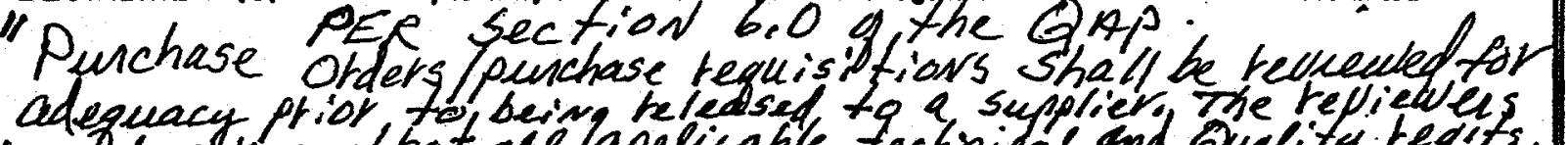

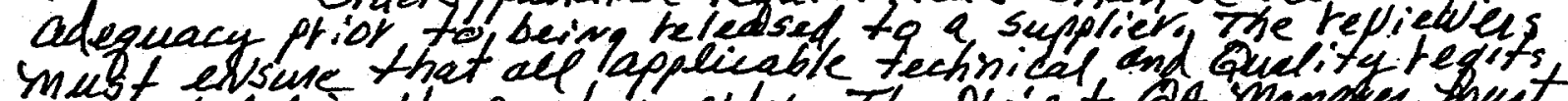

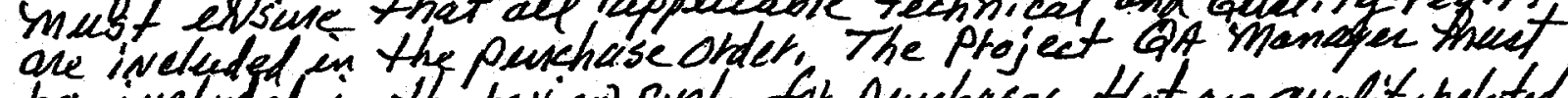
be includef in the teview cycle for puchases that are guality teloted. X FINDING NEGATIVE OBSERVATION

Puchase OSders B 940300318 (REA 39247) to Roy F. Westov INC at LiONVille, PA FOR ANolygin Sewiuns didiot convey ANalytical raguinements

0.0. 931100291 (REQ 31031) to Ecoter LSI At at Lanta, GA, basically Rame as the sbove P.O. no evidence these P. On were revieused by the Project QA Mavngor in the firsa form.

RECOMMENDATION(S) Make recammendetions you feol will be useful in resolving the finding/observation.

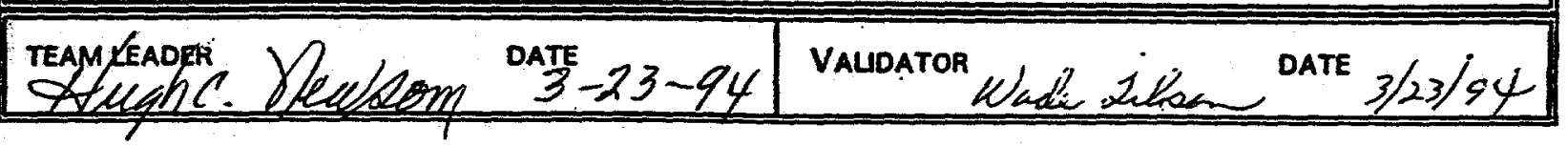




\section{MEMORANDUM}

23T-94-0043

QUA-06

CWT-94-011

May 10, 1994

TO:

Steve Thomas

FROM:

Wade Tilson

SUBJECT:

Assessment of Laboratory Performance on Total Hg Analysis

s (LEFPC-94-001)

REFERENCE: LEFPC Project

An assessment of NFS' performance on analyzing total $\mathrm{Hg}$ in LEFPC project samples was conducted on 05/03/94. Samples being analyzed were the ones taken during scooping test number 1 , conducted on $04 / 29 / 94$. The samples had been prepared by the analyst prior to the assessment. Therefore, the assessment only covered the sample analysis.

During the assessment, there was one (1) item of concern pointed out to the analyst. This concern was that the revision number of the analytical method was not being recorded in the laboratory notebook. It is important that the revision number be recorded for two reasons, i.e.; (1) There was an earlier revision of the method that did not include the techniques for analyzing solid and semisolid samples and; (2) The laboratory notebook, for this project, serves as the official analytical report.

The analyst took immediate corrective action on this item. Therefore, a written corrective action response is not requested.

Attached is a summary of the areas covered during the assessment.

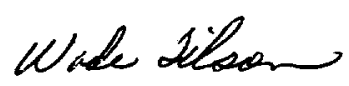

Wade Tilson

\section{CWT:ckf}

attachment

pc: Mike Rush

Doug Davis

Randy Slagle

Regulatory Services 


\section{Attachment to CWT-94-0043}

\section{SUMMARY OF O.A. ASSESSMENT (LEFPC-94-001)}

\section{Training and Qualification}

The analytical work was being performed by Mr. Doug Davis. Documentation is being maintained in the QA records file on Mr. Davis' training and qualification. It should be noted that Mr. Davis prepared the analytical method.

There are three additional people trained to analyze for total $\mathrm{Hg}$, using procedure/method NFS-RD-LAB-200, Revision 1.

\section{Procedure Implementation}

The procedure was followed during the observed analysis.

\section{Instrument Calibration}

For the analysis observed, the correlation coefficient of the calibration curve was 0.998 , which was well within the procedure requirements.

The thermometer being used in the water bath was a calibrated thermometer (TA-864 calibrated on 03/07/94; due for re-calibration on 03/07/95).

\section{Document Control}

The analytical procedure (NFS-RD-LAB-200, Revision 1), was available in the work area and was a controlled copy.

\section{Records}

Records are being maintained in a laboratory notebook. Only one concern was observed during my review of the notebook, i.e., the analytical procedure revision number was not being recorded. This concern was pointed out to the analyst and immediate corrective action was taken. No further action is required.

An excellent job is being done on maintaining the laboratory notebook. 
Page 2 -

6. Quality Control

- The calibration curve contains five (5) data points. The minimum required is four (4).

- The correlation coefficient of the calibration curve was 0.998 .

- A sample blank was analyzed. No contamination was indicated.

- A $1 \mathrm{ppm}$ check standard was analyzed before the sample batch was started. The result was $1.02 \mathrm{ppm}$.

- $\quad$ Spike duplicate samples are being analyzed as required by procedure.

\section{Handling and Storage of Soil Samples}

Soil samples were being properly handled and stored in the 131 Laboratory. No deficiencies were noted. 


\section{MEMORANDUM}

23T-94-0044

QUA-06

CWT-94-012

May 11, 1994

TO:

Steve Thomas

FROM:

Wade Tilson

SUBJECT:

Quality Assurance Assessment of LEFPC Project (LEFPC-94-002)

REFERENCE: $\quad$ NFS-Q-184, Revision 1

A QA assessment of work being performed on the LEFPC Project was conducted the week of May 2, 1994. Based on the assessment, there are several areas requiring corrective action. Attached is a summary of the areas assessed and the problems identified. Please note that items 2,5 , and 6 have deficiencies identified. You are requested to take corrective action on these items and submit a written response to QA. Corrective action must be completed prior to conducting the Pilot Demonstration. The written response is due within fifteen (15) working days from the date this report is issued.

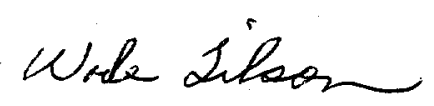

Wade Tilson

CWT/ckf

attachment(s)

pc: $\quad$ M. Rush

Regulatory Services 


\section{Attachment to CWT-94-012}

\section{Training and Qualification}

Training and qualification records for project personnel were reviewed. Records are being maintained in the OA Records File and also in the on-line T\&O system.

\section{Effective Implementation of Instructions and Procedures}

During a review of procedure/instruction implementation, the following deficiencies were noted:

- Letter of Authorization 1661B-001, Paragraph 6.3.12 provides requirements for maintaining the project logbook, including a list of "typical" information to include in the logbook. Contrary to the LOA requirements, the logbook does not include operating conditions for the trial/scooping runs. There is a mention of additional data being maintained, but it is not being maintained as a QA record.

- Page $28(04 / 27 / 94)$ of the logbook shows the results from run \#1 processing, i.e., F042594-45 (216 ppm), F042594-75 (245 ppm), P042594-150 (39.1 ppm). However, the logbook does not indicate when the samples were taken or who performed the sampling.

- The logbook indicates that two runs were identified as run \#1. (See logbook pages 28 and 30 ).

- The logbook does not indicate that a clean-out of the unit was performed between the first two runs. The logbook indicates that a clean-out was performed after the second run, but was not inspected by QC. This is contrary to the Work Plan. (See Paragraph 6.4, Page 27).

\section{Instrument Calibration}

Instruments measuring test conditions established by the Work Plan have been calibrated.

\section{Document Control}

A controlled copy of the following documents were observed to be in the 110 Building work area: 
Page 2

Attachment to CWT-94-012

- LOA 1661B-001 and Addendum 1

- VFL-WP-01, Rev. 2

- NFS-Q-184, Rev. 1

- NFS-M-055, Rev. 2

- NFS-M-056, Rev. 1

- NFS-M-057, Rev. 1

- NFS-M-058, Rev. 1

- $\quad$ LEFPC Field Changes 1, 2, 3 and 4

\section{Records}

Most of the project documentation is being maintained in the logbook. However, there are other records being generated that are only referenced in the logbook. This is a violation of Field Change \#4, Page 2, "Data will be recorded in the logbook", concerning process run data.

Additionally, LOA 1661B-001, Paragraph 7.8 requires a project daily checklist be maintained by the Process Engineer (Attachment 7 to the LOA). Based on discussions with project personnel, the checklist is not being maintained as required.

\section{Chain-Of-Custody}

Reviewed the Project Logbook for sample information required by NFS-Q-184, Paragraph 4.2.5.3, for samples taken on 05/05/94. Some of the required information was not recorded in the logbook as required, i.e., volumes or weights of the samples; purpose of sampling; method used to take the samples; where sample was sent and how transported; any site measurements made, any special precautions taken, preservatives used, if any, and any other observations made during the collection activity; signature of person(s) recording the information and observing the activity.

Reviewed the Chain-Of-Custody records for the samples taken on 05/05/94. 


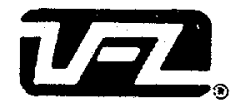

\section{VFL TECHNOLOGY CORPORATION \\ 42 LLOYD AVENUE • MALVERN, PA 19355 - (215) 296-2233 • FAX (215) 296-9545}

\section{MEMORANDUM}

\section{VIA FACSIMILE}

TO: $\quad$ Wade Tilson, NFS

FROM: $\quad$ Steve Thomas

DATE: $\quad$ May 20, 1994

SUBJ: $\quad$ Response to Quality Assurance Assessment of

LEFPC Project (LEFPC-94-002) dated May 11, 1994

\section{Corrective Actions:}

Item \#1 No response required

Item \#2 Problem - Data generated from trial run was recorded on a field data sheet (Bullet \#1) rather than log book.

Action - Operating conditions for scooping runs have been stapled in the $\log$ book.

Date Action Taken - Action will be taken on or before the date of this letter.

Prevention of Reoccurrence - All project personnel are being notified of this occurrence by receipt of this document and the QA document dated May 11, 1994. This action will facilitate correction of any errors, prevent their reoccurrence and will initiate evaluations of similar potential problem area for similar deficiencies.

(Bullet \#2) Problem - No identification of sampler and when taken

Action - Sample time and sampler will be entered in the log book and all sample log sheets will be stapled to the log book.

Date Action Taken - Action will be taken on or before the date of this letter.

Prevention of Reoccurrence - All project personnel are being notified of this occurrence by receipt of this document and the QA document dated May 11, 1994. This action will facilitate correction of any errors, prevent their reoccurrence and will initiate evaluations of similar potential problem area for similar deficiencies. 
(Bullet \#3) Problem - Incorrect run number was entered in log book.

Action - Correct run number was entered in log book.

Date Action Taken - Action will be taken on or before the date of this letter.

Prevention of Reoccurrence - All project personnel are being notified of this occurrence by receipt of this document and the QA document dated May 11, 1994. This action will facilitate correction of any errors, prevent their reoccurrence and will initiate evaluations of similar potential problem area for similar deficiencies.

(Bullet \#4) Problem - Documentation in log book does no indicate that a clean-out took place between the first two runs and no QC inspection was conducted after the second run.

Action - Documentation of the clean-out will be entered in the log book and $\mathrm{QC}$ will inspect the unit after all future runs as required in the work plan.

Date Action Taken - Action will be taken on or before the date of this letter.

Prevention of Reoccurrence - All project personnel are being notified of this occurrence by receipt of this document and the QA document dated May 11, 1994. This action will facilitate correction of any errors, prevent their reoccurrence and will initiate evaluations of similar potential problem area for similar deficiencies.

Item \#3 No response required

Item \#4 No response required

Item \#5 Problem - Material Runsheet (1661-B) is not being maintained by the Process Engineer.

Action - All data is to be entered in the log book. The Material Runsheet is no longer required due to Field Change \#6.

Date Action Taken - Action will be taken on or before the date of this letter.

Prevention of Reoccurrence - All project personnel are being notified of this occurrence by receipt of this document and the QA document dated May 11, 1994. This action will facilitate correction of any errors, prevent their reoccurrence and will initiate evaluations of similar potential problem area for similar deficiencies. 
Item \#6 Problem - Incomplete documentation of data for field sampling preservation, transportation, and sampler.

Action - All data will be entered in the log book as required. In addition, the sampling $\log (\mathrm{s})$ will be stapled in the $\log$ book.

Date Action Taken - Action will be taken on or before the date of this letter.

Prevention of Reoccurrence - All project personnel are being notified of this occurrence by receipt of this document and the QA document dated May 11, 1994. This action will facilitate correction of any errors, prevent their reoccurrence and will initiate evaluations of similar potential problem area for similar deficiencies.

cc: J. Fabrizio, VFL

B. Hoffman, VFL

M. Rush, NFS

Regulatory Services, NFS

S. Duncan, NFS

D. Davis, NFS

J. Britz, UIR

N. Stevens, UIR 


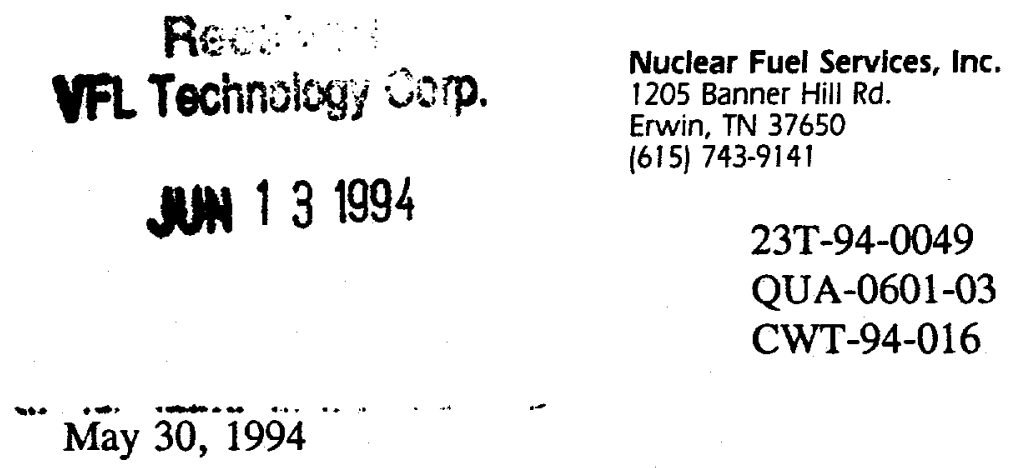

Mr. Steve Thomas

VFL Technology Corporation

42 Lloyd Avenue

Malvern, PA 19355

REFERENCE: 1) QA Assessment LEFPC-94-002, dated 05/11/94

2) VLF's response to LEFPC-94-002, dated May 20, 1994

Dear Mr. Thomas:

Reference 2 has been reviewed to determine if it meets the established criteria (NFS-Q-176) for an acceptable corrective action response. Based on the review, the deficient items included in Reference 1 were adequately addressed, with the exception of Item 5 . There seems to be some confusion between Field Change \#4 and Field Change \#6. Also, the second part of Item 5 was not addressed (maintenance of a daily checklist). When this item was identified, project management made a decision to issue a Field Change deleting the requirement for maintaining a daily checklist and to continue including applicable project information in the log book. Field Change \#6 was subsequently issued. Although issuance of a Field Change should have been more timely, the item is being "closed", since the project is essentially complete with the exception of analytical work and report writing. However, for future work, VFL and NFS project team members are reminded that approved procedures/instructions/drawings are to be strictly complied with until amended/revised with approved field changes or other approved instructions, procedures or drawings.

Attached are comments from review of your response. There is no further action required from VFL on this assessment.

If you have any questions concerning the above information, please do not hesitate to contact me.

Sincerely,

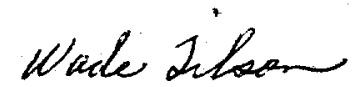

Wade Tilson

attachment

Project QA Manager 


\section{Attachment to CWT-94-016}

Item \#2

(Bullet \#1)

(Bullet \#2)

(Bullet \#3)

(Bullet \#4)

Item $\# 5$

Item $\# 6$
Acceptable; action was taken as committed.

The corrective action was acceptable.

Acceptable

Acceptable

The response does not totally address the problems identified. Field Change \#4 (not Field Change \#6) deleted the requirement that process run information be maintained on Runsheet 1661B-1 and stated that "data will be recorded in the logbook". However, run data was not being recorded in the logbook but instead recorded on a data sheet designed by VFL and UIR. From a quality assurance point of view, this is acceptable provided the data was later included in the logbook required by Field Change \#4. Based on a follow-up review, the data sheets have now been stapled in the logbook.

The response does not address the second deficiency identified in Item \#5, i.e., maintenance of a project daily checklist as required by LOA 1661B001 , Paragraph 7.8. However, Field Change \#6 was issued deleting the requirement to maintain the checklist. This item is "closed".

Sampling information recorded in the logbook subsequent to $05 / 05 / 94$ included applicable information. This item is "closed". 


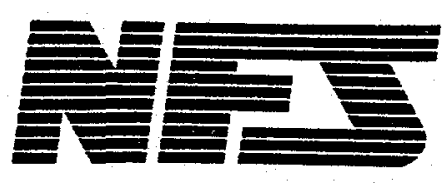

March 25, 1994

Mr. Joe Fabrizio, Project Manager

VFL Technology Corporation

42 Lloyd Avenue

Malvern, Pennsylvania 19355

REFERENCE: 1) LEFPC Project

2) NFS-Q-184, Revision 1

SUBJECT: $\quad$ Quality Assurance Manager's Report (Monthly Report \#1)

Dear Mr. Fabrizio:

Enclosed for your information and use is the Quality Assurance Manager's Report for March 1994 (Monthly Report \#1). This report is required by Reference \#2 above.

If you have any questions or comments concerning this report, please do not hesitate to contact me.

Sincerely,

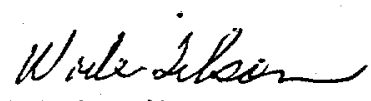

Wade Tilson

LEFPC Project QA Manager

\section{CWT:ckf}

enclosure(s)

Distribution:

Mike Rush

Steve Thomas

Wade Tilson

Chron 


\subsection{Periodic Assessment of Measurement Data Accuracy, Precision, and Completeness}

In January of this year, NFS received the analytical results from the soil characterization samples sent to EcoTek, LSI in Atlanta, Georgia. The analytical data was reviewed for accuracy, precision, completeness, and reported to you on 01/14/94. However, during a subsequent review of the statistical calculations, performed by NFS, it was determined that an error had been made in the confidence internal calculations. The error was corrected and a revised report was issued to you on 01/19/94. Both EcoTek, LSI and MMES' analytical results confirmed that the soil is contaminated with $\mathrm{Hg}$ at levels such that a meaningful thermal desorption study can be performed.

\subsection{Results of QA Assessments/Audits}

MMES performed a OA assessment on the LEFPC Project, March $21-23,1994$. During the assessment, three findings were reported. The first finding was related to a lack of training documentation for certain project personnel. The second finding was concerning the calibration accuracy of three (3) type $K$ thermocouples, i.e., the thermocouples exceeded $\pm 1 \%$ accuracy at the lower check point. The third finding was related to procurement document control, i.e., Purchase Orders issued to Roy F. Weston Laboratory and EcoTek, LSI Laboratory did not convey analytical requirements correctly. A copy of the finding sheets are attached for your information.

Corrective actions are being taken for each finding. The next QA Manager's report will include a summary of the corrective actions taken.

In addition to the QA Assessment, an operational readiness review was conducted by MMES on March 23, 1994. During the QA assessment and operational readiness review, MMES identified other areas requiring corrections prior to initiating the LTTD trial runs. These items were formally transmitted to you in a letter from Lisa L. Cobb (MMES) on March 24, 1994. MMES has requested that all items be corrected prior to initiating the first test run.

NFS and VFL are in the process of correcting all items identified in the MMES letter. Next months QA Manager's report will include a summary of the actions taken.

\subsection{Significant QA Problems and Recommended Solutions}

To date, there have been no significant $Q A$ problems identified.

NFS and VFL are in the process of taking corrective actions requested by MMES in the QA assessment and operational readiness review.

\subsection{Resolutions of Previously Stated Problems}

There were no previously stated problems. 
FINDING/OBSERVATION REPORT

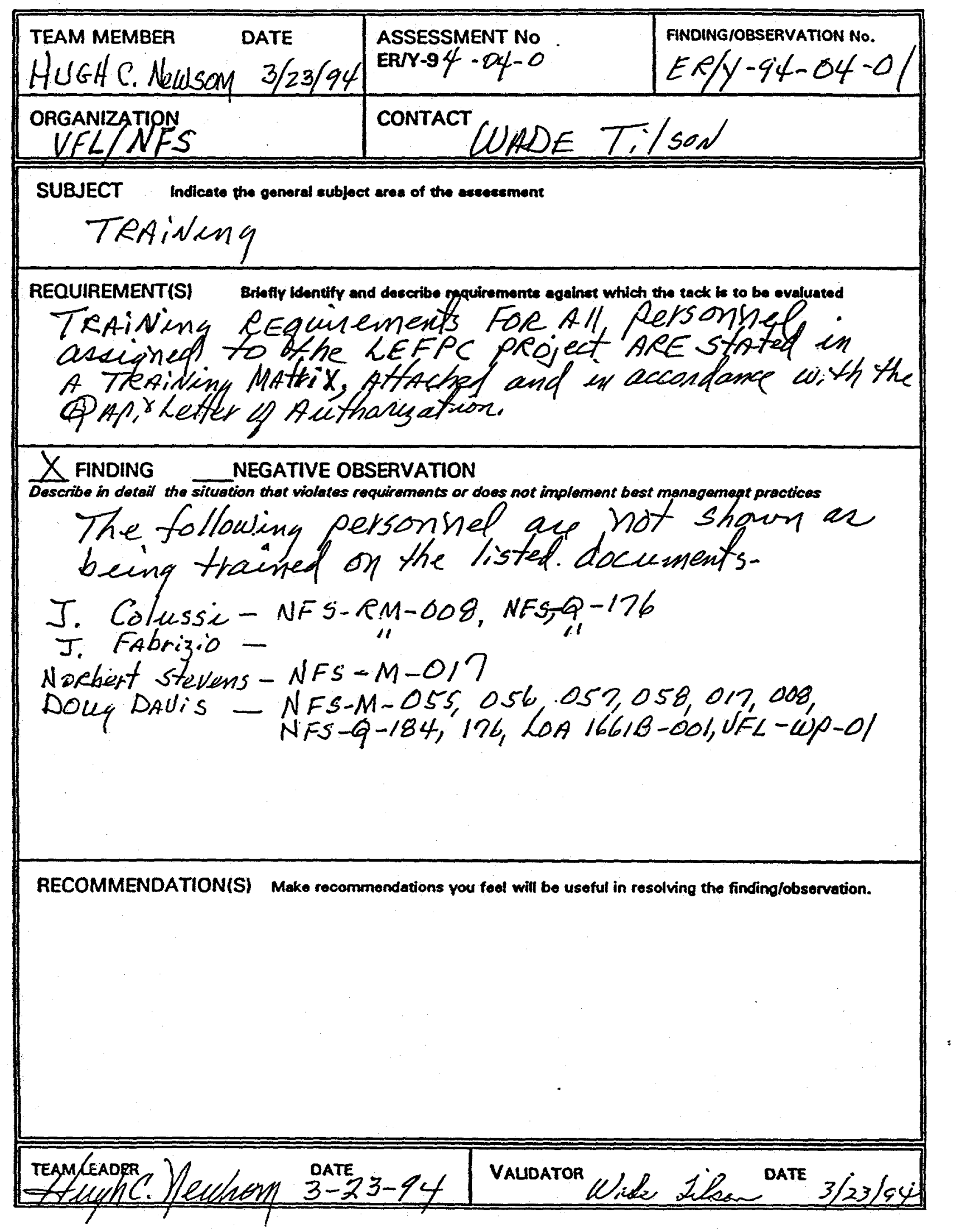


FINDING/OBSERVATION REPORT

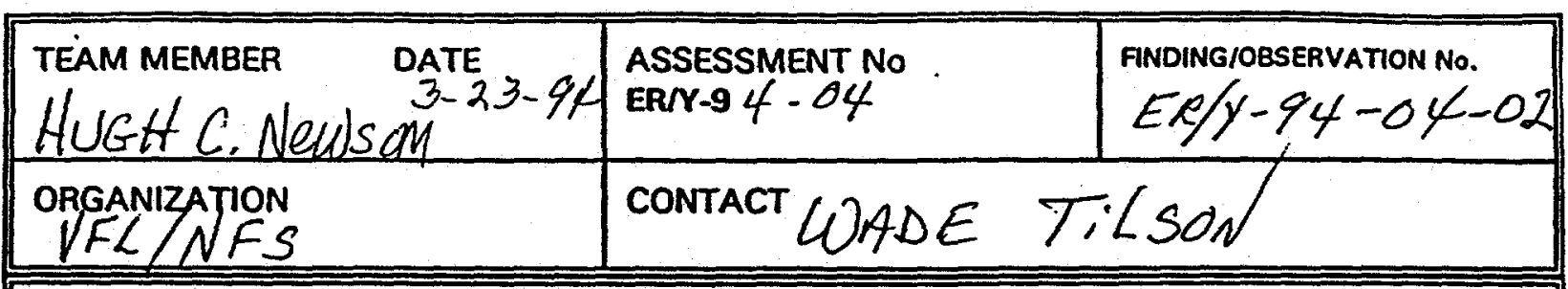

SUBJECT

CAlibration of INStRuMENts

REQUIREMENT(S) Briefly Identify and describe requirements against which the tack is to be evaluated

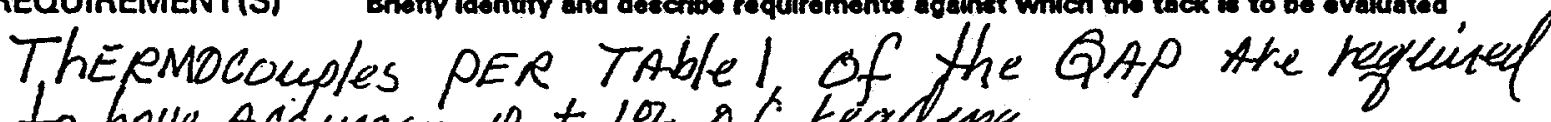
to have Acc macy \& $\pm 1 \%$ of reading

$X$ FINDING

NEGATIVE OBSERVATION

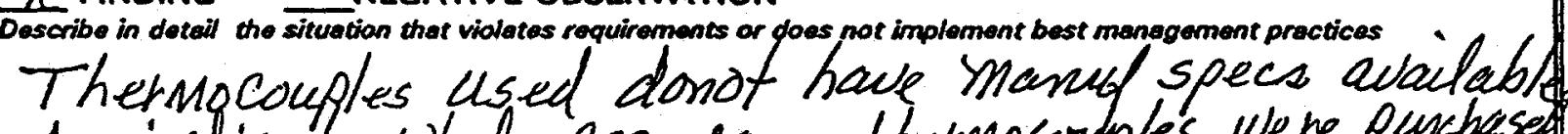
to indicate what Accuracy. themplongles we ne purchased ac. All thermocouples were wealibtated with a standard

An $\pm 10^{\circ} \mathrm{F}$ Accuracy. Three Thermocouples have readings (Calibrated recants) of the following: $3709 \ldots 212^{\circ} \mathrm{f}$ - CAlibrated reading $217^{\circ}$ $3711-212^{\circ} f-$ - Calibrated reading $217^{\circ}$ $3212-\ldots 212^{\circ} \mathrm{f}-\mathrm{CA}_{4}$ librated reading $217^{\circ}$

These readings exceed 1\%o Accicuacy

Assumption was mads the thermocouples hilda

RECOMMENDATION(S) Make recommendations you fool will be useful in resolving the finding/observation.

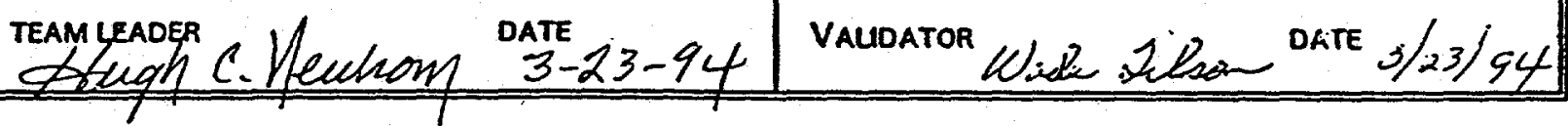


FINDING/OBSERVATION REPORT

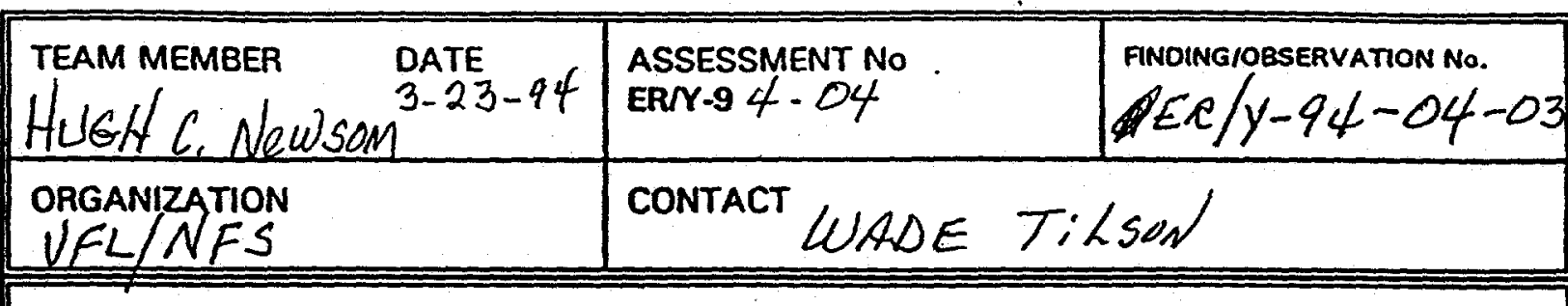

Procurement Doc um ant Control

REQUIREMENT (S) Briefly Identity and describe requirements against which the tack is to be evaluated

"Purchase orders purchase requisitions shell

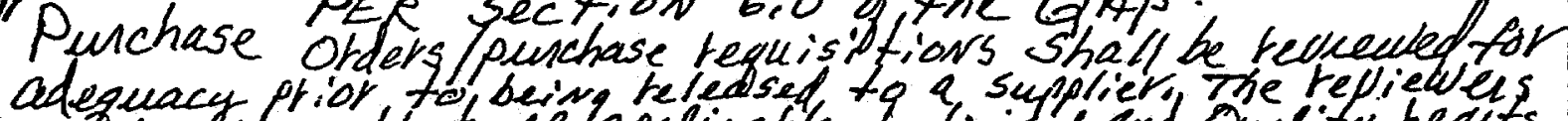

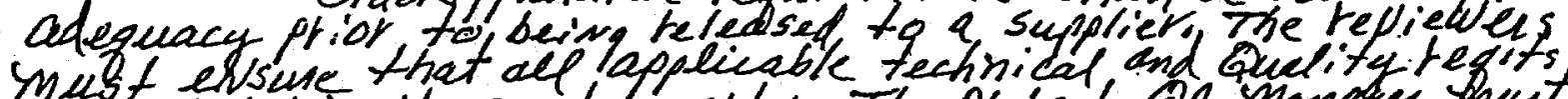
are indented in the purchase older the project QA manse shut

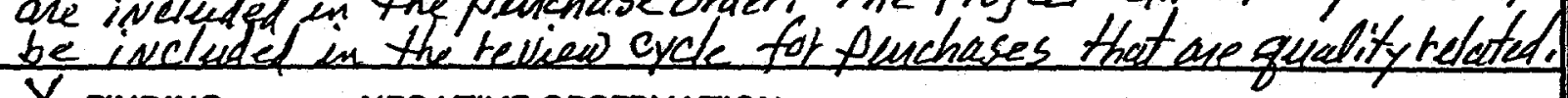
X FINDING NEGATIVE OBSERVATION

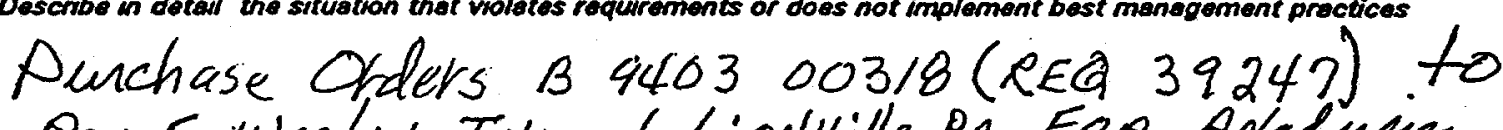

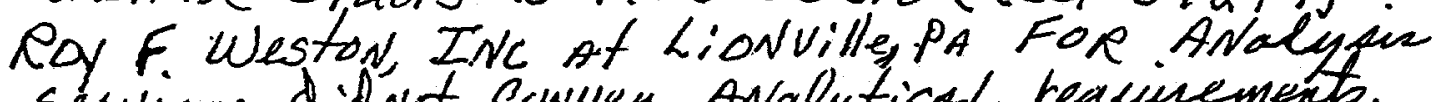

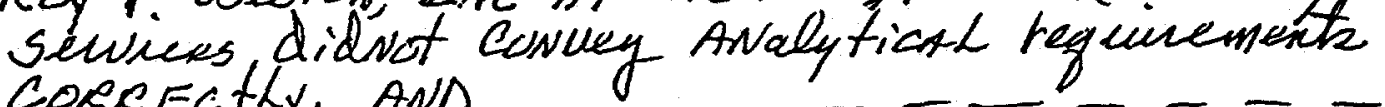
CORRECTLY AND

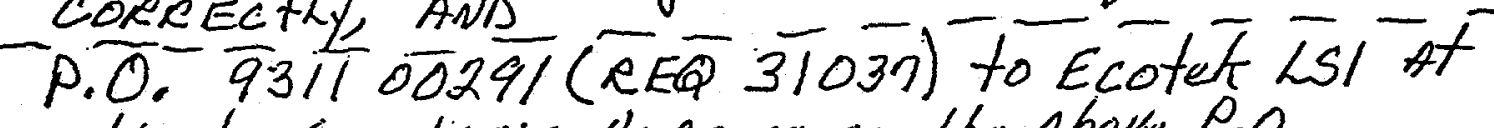
atlanta, Ga, basically same as the above P.O. no evidence these P. On were reviundel by the Project QA Manager in the final form.

RECOMMENDATION(S) Make recommendations you foal will be useful in resolving the findinglobservation.

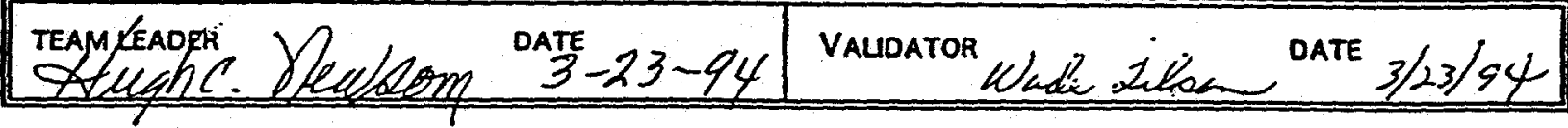




\subsection{Periodic Assessment of Measurement Data Accuracy, Precision, and Completeness}

An assessment of NFS' performance on analyzing total $\mathrm{Hg}$ in LEFPC project samples was conducted on May 3, 1994. During the assessment, measurement data was reviewed for accuracy, precision and completeness. There was one item of concern pointed out to the analyst. The concern was that the analytical method revision number was not being recorded in the laboratory notebook. The reasons for the concern was that: (1) There was an earlier revision to the method that did not include techniques for analyzing solid and semi-solid samples and; (2) The laboratory notebook, for this project, serves as the official analytical report for trial runs. Immediate corrective action was taken. No further action was required.

\subsection{Results of QA Assessments/Audits}

Two (2) QA assessments were performed during the month of May. The first assessment (LEFPC-94-001) was on NFS's performance on analyzing total $\mathrm{H}_{2}$ in the project samples taken during trial runs. (See 1.0 above). The second assessment covered the following areas:

- Training and Qualification

- Effective Implementation of Instructions and Procedures

- Instrument Calibration

- Document Control

- Records

- Chain-of-Custody

Deficiencies were reported in three (3) of the six areas assessed. In general, the deficiencies were related to record keeping and/or lack of documentation in the project log book. A copy of both assessment reports (LEFPC-94-001 and LEFPC-94-002) were previously provided to you and Mr. Steve Thomas.

Mr. Thomas provided a written corrective action response to LEFPC-94-002, as requested. The response was evaluated for acceptance (Refer to letter CWT-94-016 to Steve Thomas, dated 05/30/94). All items to date have been "closed".

\subsection{Significant QA Problems and Recommended Solutions}

There were no significant $Q A$ problems for this reporting period. Refer to $Q A$ assessment Reports LEFPC-94-001 and LEFPC-94-002 for deficiencies identified during this reporting period. The deficiencies identified to date should not have an adverse affect on the quality of the project results.

The pilot demonstration was completed on $05 / 12 / 94$, and the samples were shipped to off-site laboratories for the required analyses on 05/16/94.

\subsection{Resolutions of Previously Stated Problems * *.}

There are no "open" items from previous reports. 


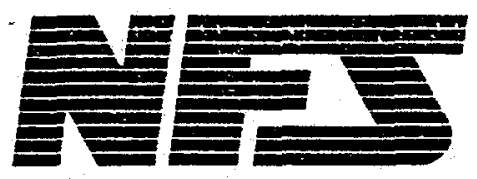

$$
\text { RECEIVED UUL } 19 \text { SOSF }
$$

Nuclear Fuel Services, Inc.

July 11,1994

Mr. Joe Fabrizio, Project Manager

VFL Technology Corporation

42 Lloyd Avenue

Malvern, Pennsylvania 19355

REFERENCE: 1) LEFPC Project

2) NFS-Q-184, Revision 1

SUBJECT: $\quad$ Quality Assurance Manager's Report (Monthly Report \#4)

Dear Mr. Fabrizio:

Enclosed for your information and use is the Quality Assurance Manager's Report for June 1994 (Monthly Report \#4). This report is required by Reference $\# 2$ above.

If you have any questions or comments concerning this report, please do not hesitate to contact me.

Sincerely,

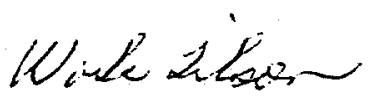

Wade Tilson

LEFPC Project QA Manager

CWT:ckf

enclosure(s)

pc: M. Rush

S. Thomas (VFL)

W. Tilson

Regulatory Services 


\section{QUALITY ASSURANCE MANAGER'S REPORT \#4 \\ JUNE 1994}

\subsection{Periodic Assessment of Measurement Data Accuracy, Precision, and Completeness}

Data packages RFW 9405L630 and RFW 9405L624, from Roy F. Weston Laboratory were reviewed for compliance to the purchase order requirements, $Q A$ Plan requirements, and also to determine if they met SW-846 method 8280 (The Analyses of Polychlorinated Dibenzo-pDioxin and Polychlorinated Dibenzolfurans). Based on the review, it was determined that the analytical packages were acceptable.

A simiiar review will be made on the analytical packages from EcoTek LSI.

2.0 Results of QA Assessments/Audits

There were no $Q A$ assessments/audits conducted for this reporting period.

3.0 Significant OA Problems and Recommended Solutions

None for this reporting period.

\subsection{Resolutions of Previously Stated Problems}

There are no "open" items from previous reports. 


\section{READINESS REVIEW}

for the

\section{LEFPC THERMAL DESCRIPTION DEMONSTRATION}

Completed March 29, 1994

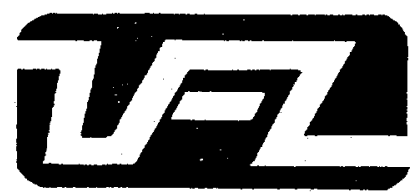

VFL Technology Corporation

42 Lloyd Avenue

Malvern, PA 19355

(610) $296-2233$ 


\section{READINESS REVIEW}

for the

\section{LEFPC THERMAL DESCRIPTION DEMONSTRATION}

Completed March 29, 1994

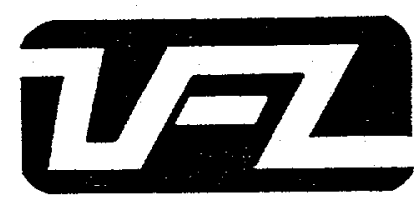




\section{OUALITY ASSURANCE}

1) Have wipe test(s) been completed assuring cleanliness of unit?

2) Have project personnel received training on project procedures and instruction?

3) Is personnel training documented on the T\&Q System?

4) Have the required instruments been calibrated?

5) Has a project log book been set up and made available to project personnel for required entries?

6) Is a fire proof filing cabinet available for QA Record Storage?

7) Was the bench-scale study successfully completed and documented?

8) Are process/test runsheets available for the start of work?

Please check the

appropriate answer

\section{SAFETY}

9) Are Lock-out and Tag-out equipment available?

10) Is all personnel safety equipment in place and personnel properly trained to use it?

11) Has the final NFS IAC inspection for startup been completed and approved by all 4 Safety Department disciplines?

12) Has the Radiation Work Permit been written and approved?

13) Have all required training (radiological and industrial) been completed?

14) Have all addressed equipment safety concerns been completed?

15). Is all industrial safety equipment (mercury detectors, etc.) in place and working?
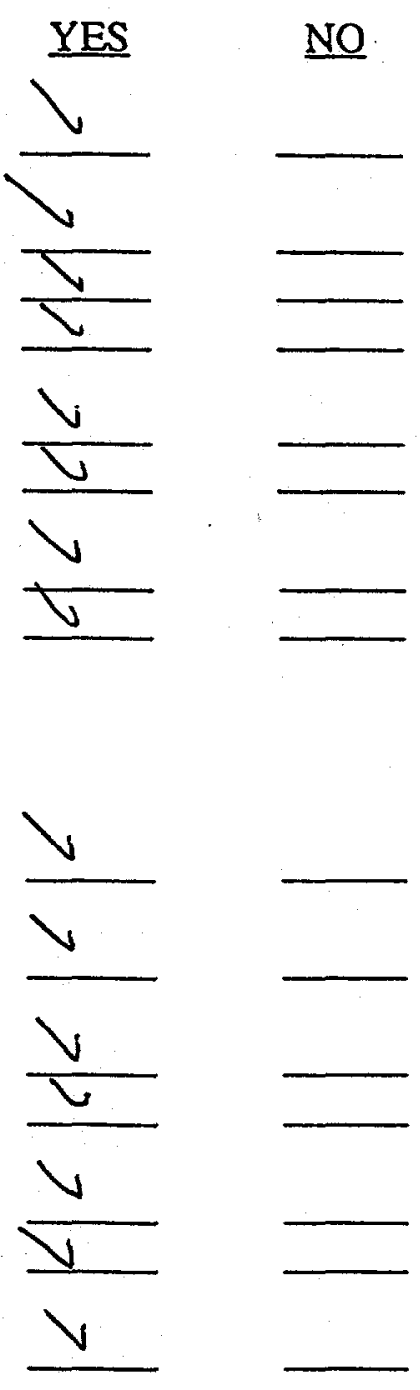

\section{GENERAL}

16) Has additional storage capacity been added to the condenser to collect condensate from the $3,000 \mathrm{lb}$ run?

17) Has the airlock been installed on unit?

18) Have the Hot Oil System pressure releases been properly piped to overflow collection vessel(s)?

19) Are all required project personnel on site and prepared to perform their appropriate tasks?

20) Has Therminol 75 been added to the proper level in the system? Dourtiken $G$ (rest 3-27-44)

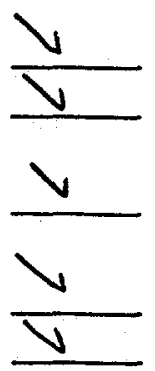




\section{CHECKLIST FOR LEFPC PROJECT}

(cont'd)

21) Has the oil system been bled of gas?

22) Has the off-gas (with cold trap) been connected?

23) Has the compressed air been connected?

24) Has the $\mathrm{N}_{2}$ been connected?

25) Has the electricity been connected?

26) Has the steam been connected?

27) Has the cooling $\mathrm{H}_{2} \mathrm{O}$ been connected?

28) Has the condensate discharge system been connected?

29) Is the discharge chute connected to the drum?

30) Is the soil handling equipment (conveyor, mixing box, shredder) in place and ready to run?

31) Are all control systems in proper working order?

32) : Are scoops (plastic and metal) available?

33) Are sample bottles available?

34) Are reagents available?

35) Is the dry ice/alcohol cold trap ready?

36) Is seal water (for cooling purposes) hooked to the hot oil pump?

37) Are the required sampling supplies, i.e., sample containers, scoops, labels, seals, chain-of-custody forms, and shipping containers, available?

Please check the appropriate answer

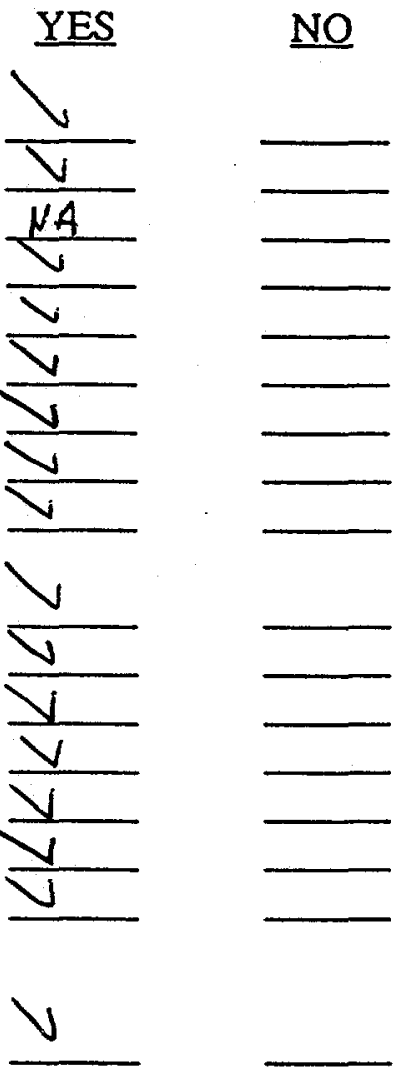

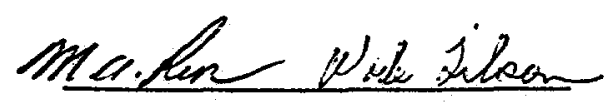

NFS REPRESENTATIVE
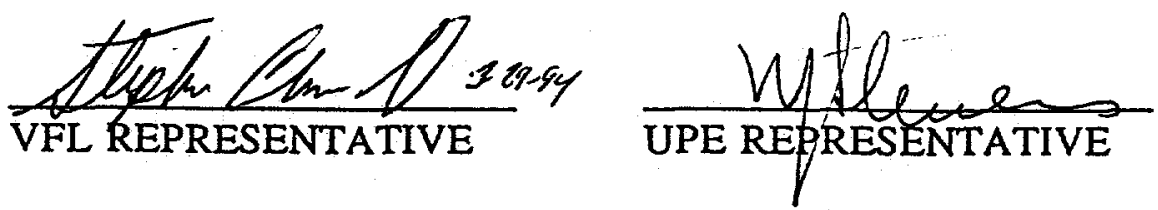
To: $\quad$ M.A.Rush

From: $\quad$ J.D.Davis

Date: $\quad$ April 25, 1994

Subject: Training of Personnel to Perform Mercury Analyses for LEFPC Project

The following have received training and are qualified to analyze LEFPC samples for mercury per NFS-RD-LAB-200, Revision 1, MERCURY IN AQUEOUS SOLUTIONS AND SOLID AND SEMISOLID SAMPLES.

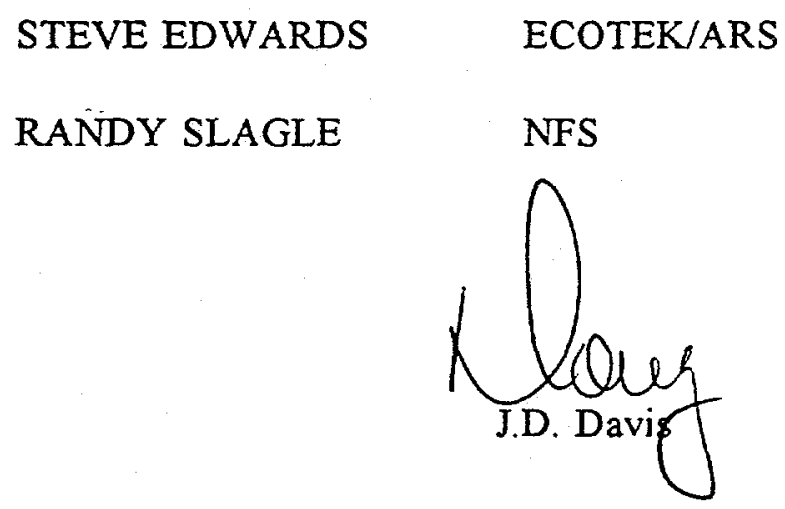

cc: Wade Tilson 
To: M.A. Rush

From: J.D. Davis

Date: March 25, 1994

Subject: Training of Designated Samplers for LEFPC Project

I hereby certify that the following personnel have received training in the correct techniques for collecting the samples specified in NFS-M-055, "Sampling and Analysis Plan, Lower East Fork Poplar Creek Project, Thermal Desorption Pilot Demonstration." Specifically, those persons (listed in the table below) were trained in the collection of soil, condensate, field blank, and duplicate samples for both on-site and off-site analyses. Also included, was training in the correct techniques for sampling the cold-trap condenser and taking swipe samples.

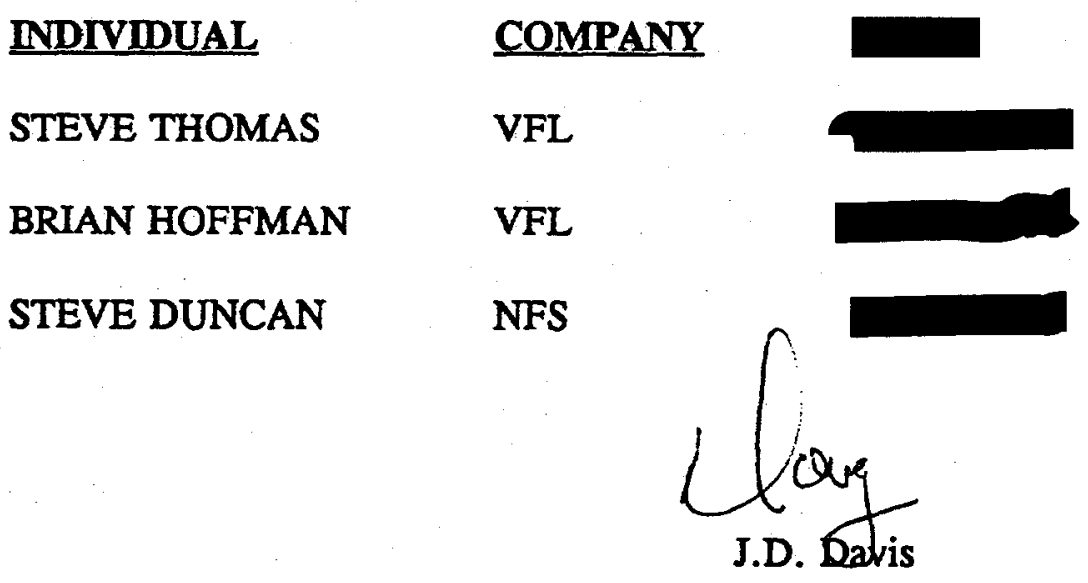

cc: Steve Thomas

Wade Tilson 
LEFPC PROJECT TRAINING RECORD

SOP/OA SIGNOFF

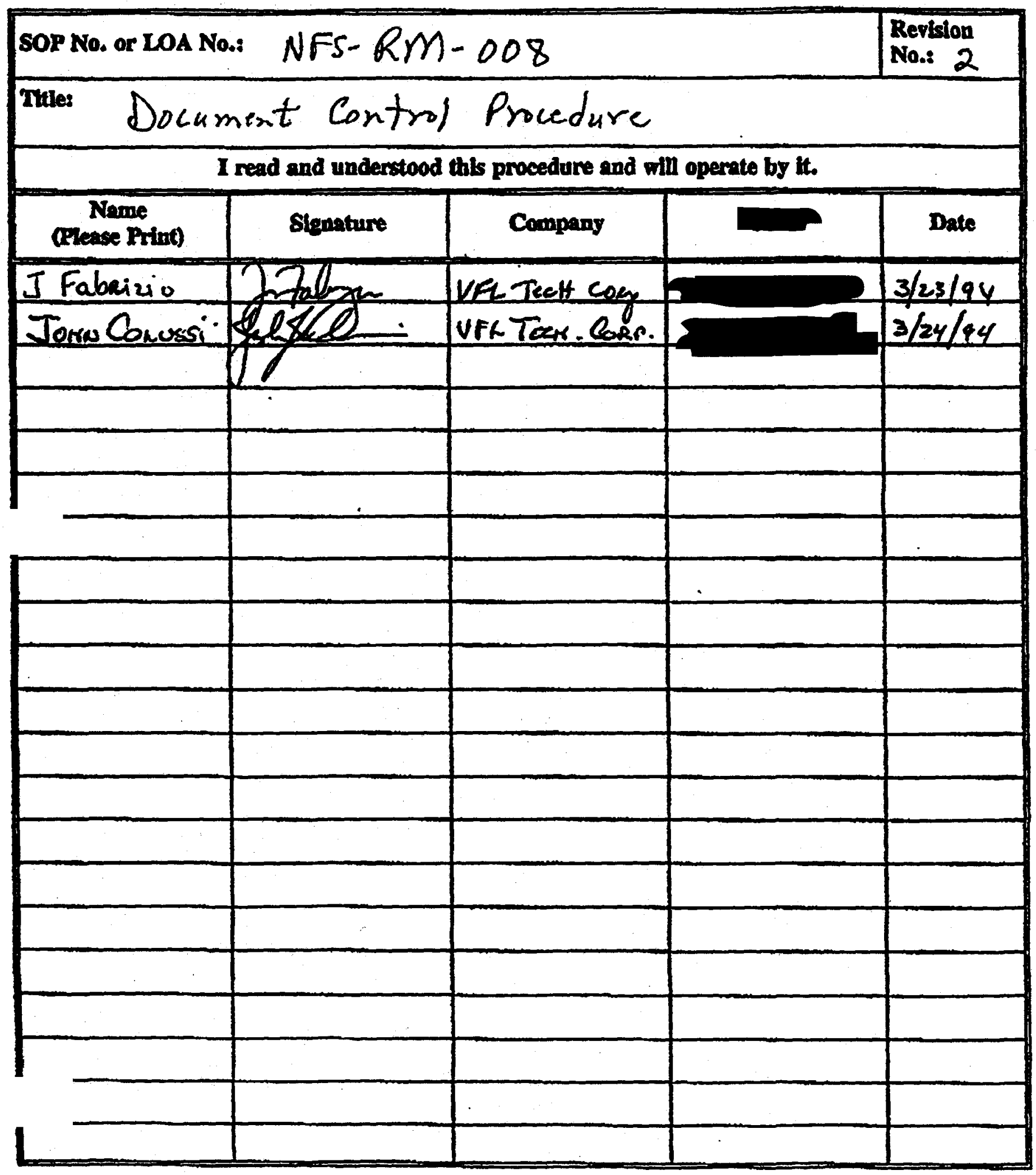




\section{LEFPC PROJECT TRAINING RECORD}

\section{SOP/LOA SIGNOFF}

\begin{tabular}{|r|r|r|r|r|}
\hline SOP No. or LOA No: NFS-Q-176 & Revision \\
No.: 0
\end{tabular}




\section{LEFPC PROJECT TRAINING RECORD}

\section{SOP/LOA SIGNOFF}

\begin{tabular}{|c|c|c|c|}
\hline \multicolumn{3}{|c|}{ SOP No. or LOA No.: NFS-m-1? } & $\begin{array}{l}\text { Revision } \\
\text { No.: } 0 / 4\end{array}$ \\
\hline \multicolumn{4}{|c|}{ Title: Calibration System Manual } \\
\hline \multicolumn{4}{|c|}{ I read and understood this procedure and will operate by it. } \\
\hline $\begin{array}{c}\text { Name } \\
\text { (Please Print) }\end{array}$ & Signature & Company & Date \\
\hline Sreve Twans & $\operatorname{Ln} 2$ & VFC & $3-24.94$ \\
\hline NIStEVENS & Whlenens & Jimsens & $3-24-9 v$ \\
\hline & & & \\
\hline & & & \\
\hline & & & \\
\hline & & & \\
\hline & & & \\
\hline & & & \\
\hline & & & \\
\hline & & & \\
\hline & & & \\
\hline & & & \\
\hline & & & \\
\hline & & & \\
\hline & & & \\
\hline & & & \\
\hline & & & \\
\hline & & & \\
\hline & & & \\
\hline & & & \\
\hline
\end{tabular}




\section{LEFPC PROJECT TRAINING RECORD}

SOP/LOA SIGNOFF

\begin{tabular}{|l|l|l|l|l}
\hline SOR No. or LOA No.: NFS-M-055ion & $\begin{array}{l}\text { No.: } 2 \\
\text { No. }\end{array}$ \\
\hline
\end{tabular}

Title: Sampling and Analysis Plan LEFPC Project Thermal Desorption Pilot Demonstration

I read and understood this procedure and will operate by it.

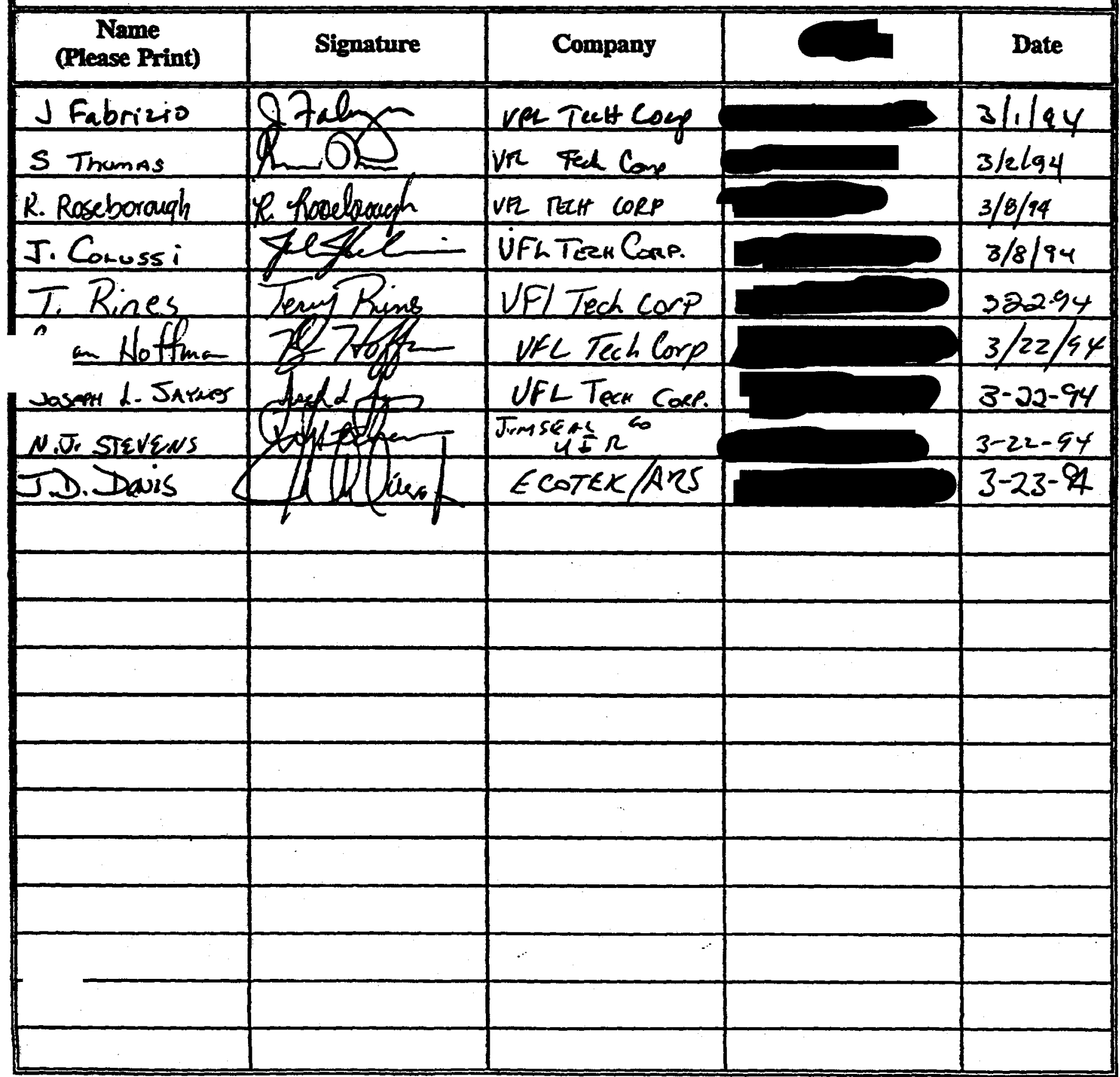




\section{LEFPC PROJECT TRAINING RECORD}

\section{SOP/LOA SIGNOFF}

\begin{tabular}{|c|c|c|c|}
\hline SOP No. or LOA No. & $N F S-m-0$ & & $\begin{array}{l}\text { Revision } \\
\text { No.: } 1\end{array}$ \\
\hline $\begin{array}{r}\text { Tritle: Transport } \\
\text { Pilot D }\end{array}$ & $\begin{array}{l}\text { ion Plan L } \\
\text { onstration }\end{array}$ & FPC Project & trion \\
\hline & ad and understood & this procedure and wh & \\
\hline $\begin{array}{c}\text { Name } \\
\text { (Please Print) }\end{array}$ & Signature & Company & Date \\
\hline$J$ Fabrizio & & VPL TenH Cone & $3 / 1 / 94$ \\
\hline S Thumas & & Vfe Tech Conn & $3 / 2 / 04$ \\
\hline R. Roseborough & iselorougl & UR TRH CORP & $3 / 0 / 94$ \\
\hline J. Cowssi & & UfLT Teen Coxp. & $3 / 8 / 94$ \\
\hline I Rines & $D$ & VF/ Tech cors & $3-22-9$ \\
\hline lan Noffman & Hoftma & VFL Tech Corp & $3 / 22 / 94$ \\
\hline Sosepth L. Snines & 0 & UFL TREA CoN & $3-22-94$ \\
\hline NJistivens & & Jinsentis & $3-22-94$ \\
\hline Ja Dais & & EcoteK/ARs & $3-23-94$ \\
\hline & & & \\
\hline & & & \\
\hline & & & \\
\hline & & & \\
\hline & & & \\
\hline & & & \\
\hline & & & \\
\hline & & & \\
\hline & & & \\
\hline & & & \\
\hline & & & \\
\hline & & & \\
\hline
\end{tabular}


LEFPC PROJECT TRAINING RECORD

SOP/LOA SIGNOFF

SOP No. or LOA No.: NFS- $m-057$

Title: Waste Management Plan LEFPC Project Thermal Desorption Pilot Demonstration

I read and understood this procedure and will operate by it.

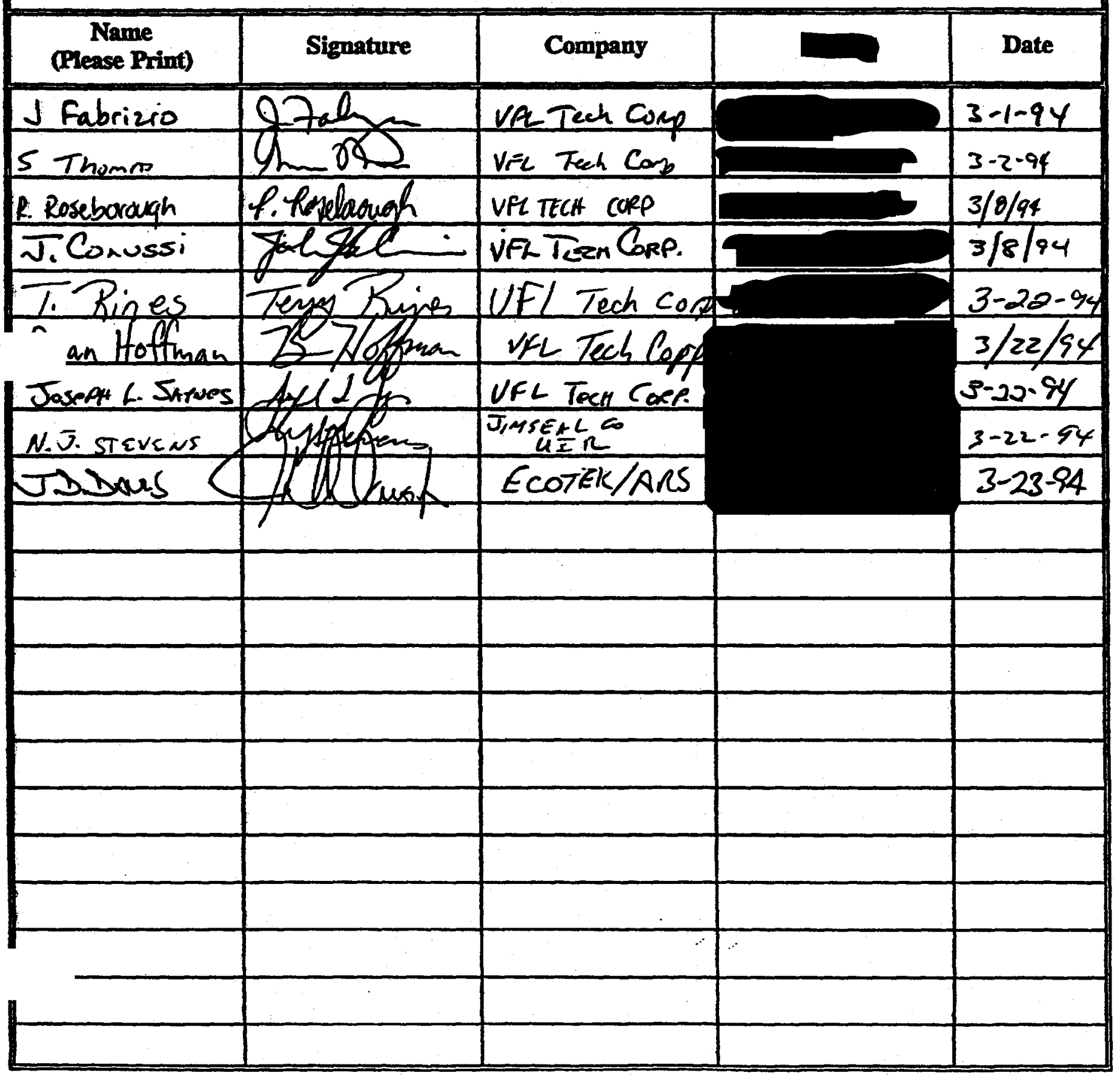




\section{LEFPC PROJECT TRAINING RECORD}

\section{SOP/LOA SIGNOFF}

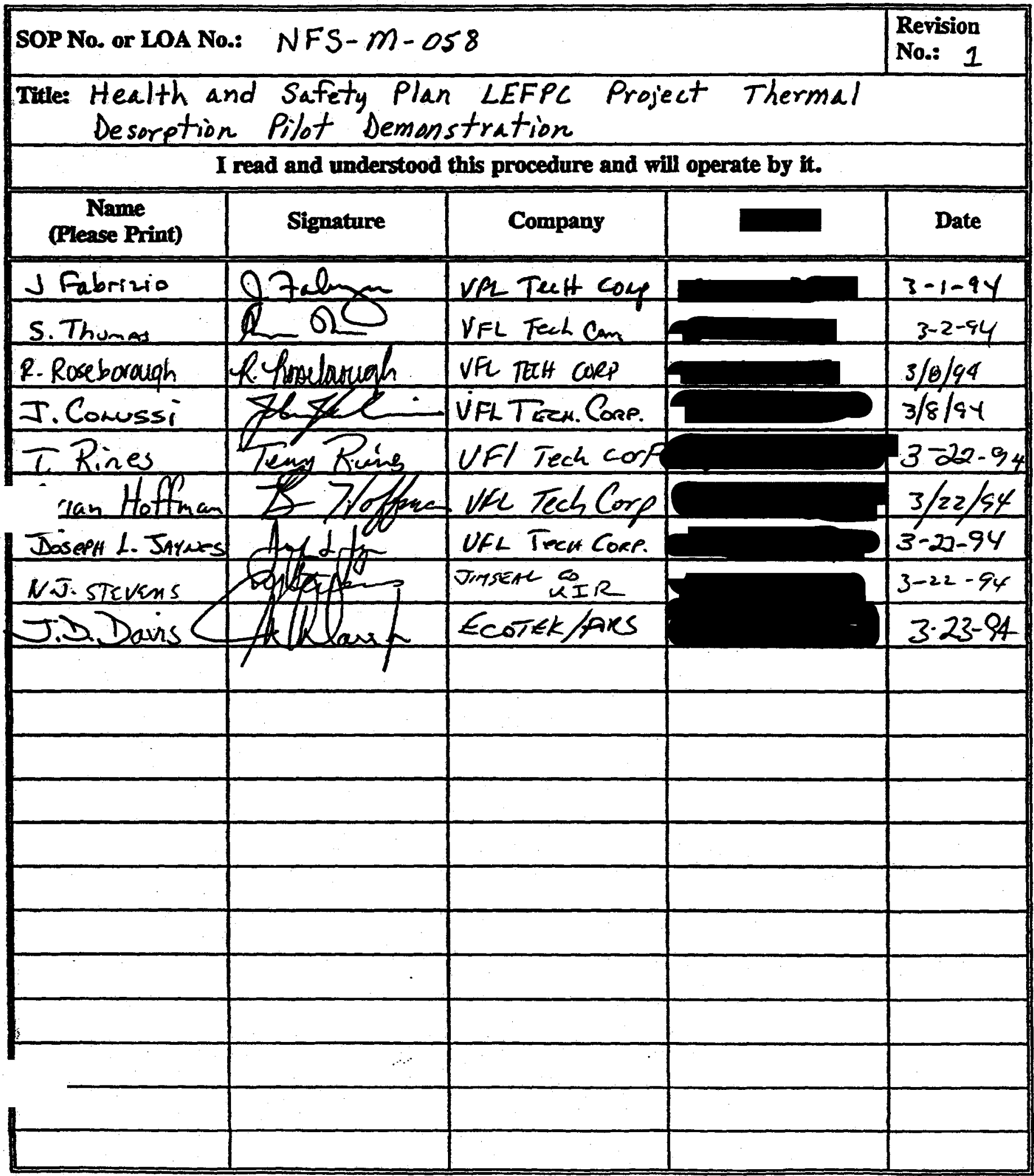




\section{LEFPC PROJECT TRAINING RECORD}

SOP/LOA SIGNOFF

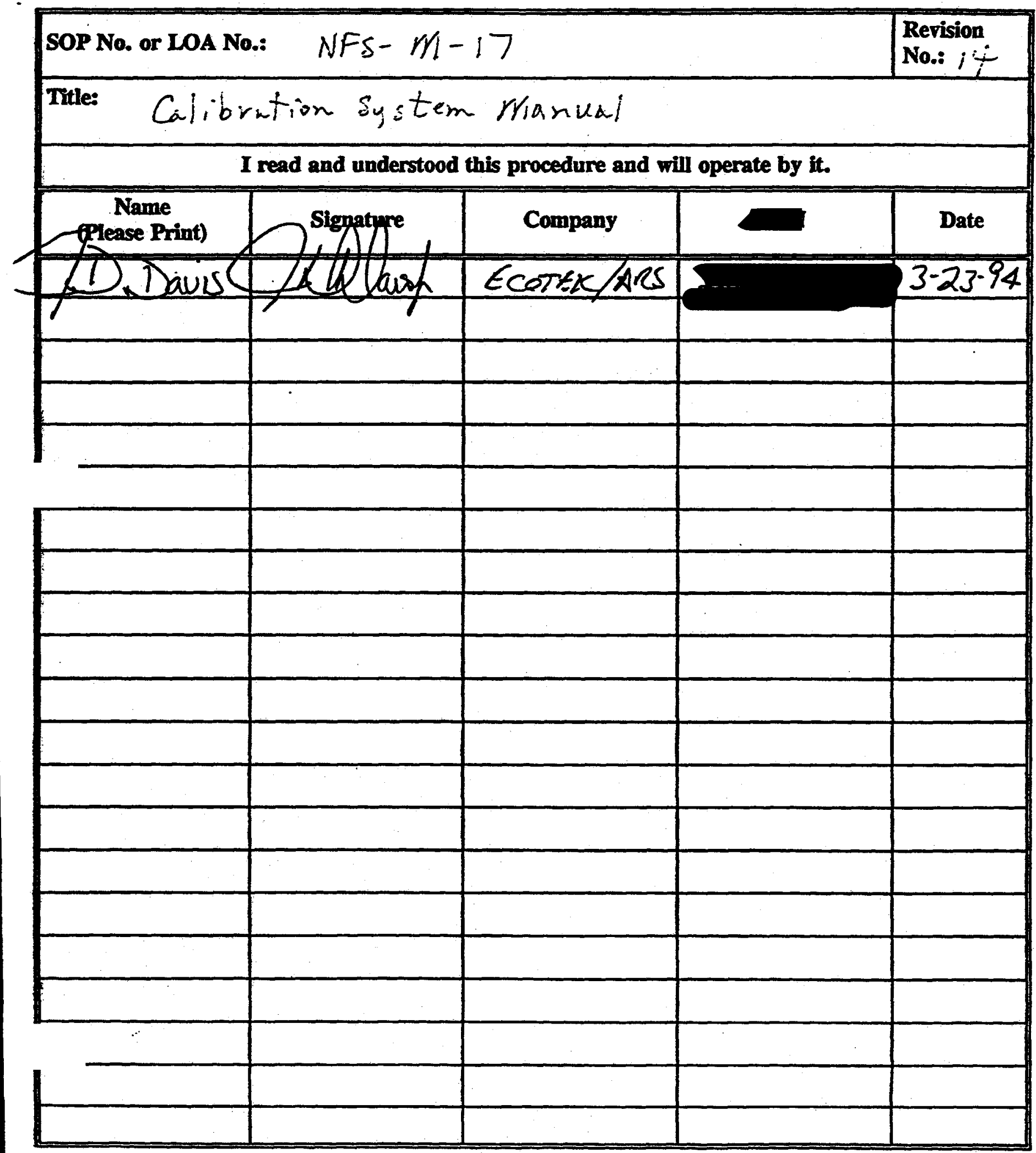


LEFPC PROJECT TRAINING RECORD

SOPILOA SIGNOFF

\begin{tabular}{|l|l|l|l|l|}
\hline SOP No. or LOA No.: NFS-RMI-OO B & Revision \\
No.: 2
\end{tabular}




\section{LEFPC PROJECT TRAINING RECORD}

\section{SOP/LOA SIGNOFF}

SOP No. or LOA No: NFS-RM-OCB
Title: Decument Control Precedure

I read and understood this procedure and will operate by it.

\begin{tabular}{|c|c|c|c|}
\hline $\begin{array}{c}\text { Name } \\
\text { (Please Print) }\end{array}$ & Signature & Company & Date \\
\hline Steue Thimas & $\alpha-2$ & VFL & $324-54$ \\
\hline N.J.Sterkas & ustener & Gimsem a & $3-2 y-9 y$ \\
\hline & & & \\
\hline & & & \\
\hline & & & \\
\hline & & & \\
\hline & & & \\
\hline & & & \\
\hline & & & \\
\hline & & & \\
\hline & & & \\
\hline & & & \\
\hline & & & \\
\hline & & & \\
\hline & & & \\
\hline & & & \\
\hline & & & \\
\hline & & & \\
\hline & & & \\
\hline & & & \\
\hline
\end{tabular}


LEFPC PROJECT TRAINING RECORD

SOP/LOA SIGNOFF

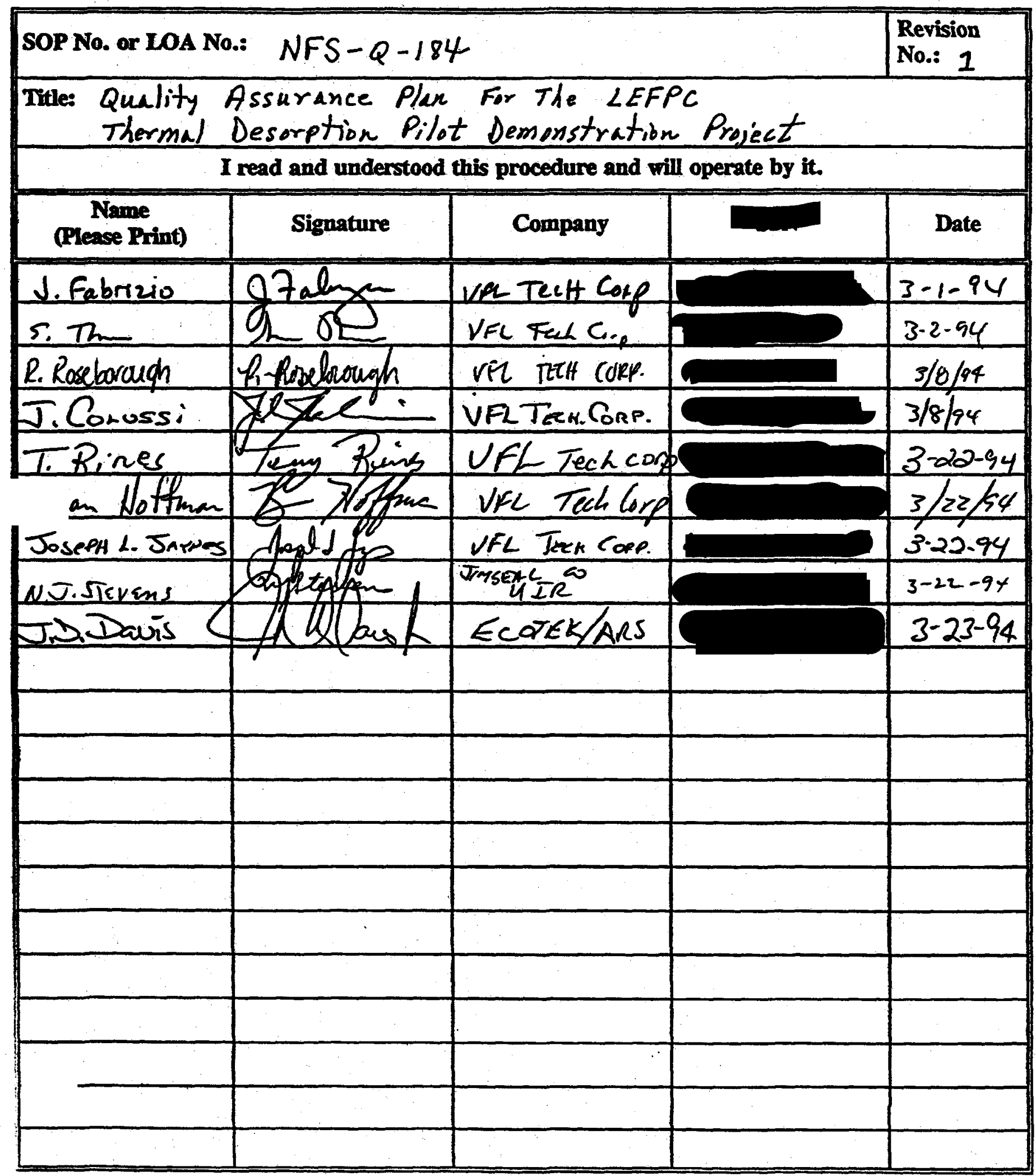




\section{LEFPC PROJECT TRAINING RECORD}

SOP/LOA SIGNOFF

SOP No. or LOA No.: NFS $-Q-176$
Tite: Corrective Actiin Procedure

I read and understood this procedure and will operate by it.

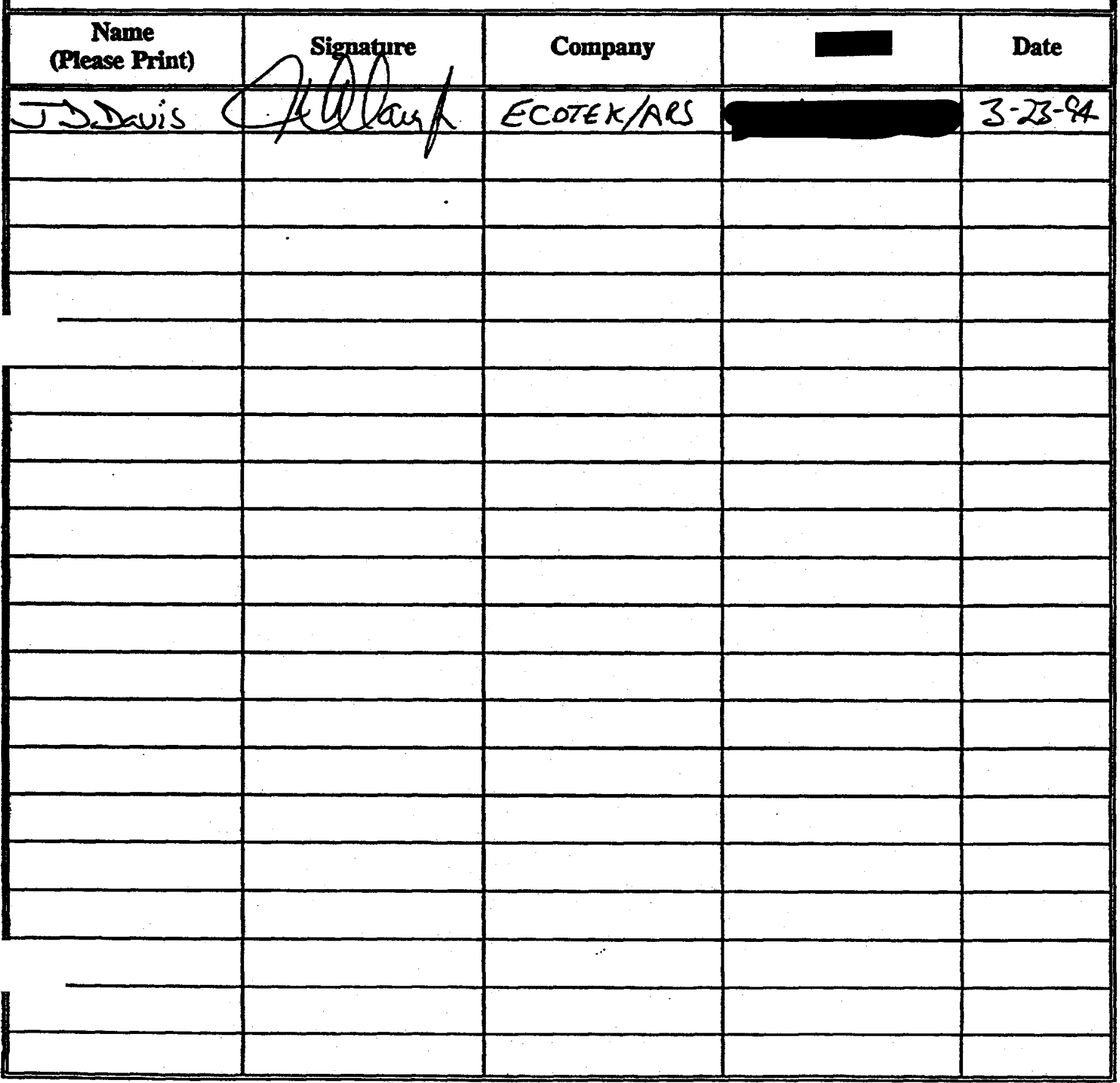




\section{LEFPC PROJECT TRAINING RECORD}

\section{SOP/LOA SIGNOFF}

SOP No. or LOA No.: NFS-Q-176

Revision

No.: $00 C$

Title: Cirrective Action Procedure

I read and understood this procedure and will operate by it.

\begin{tabular}{|c|c|c|c|}
\hline $\begin{array}{c}\text { Name } \\
\text { (Please Print) }\end{array}$ & Signature & Company & Date \\
\hline StEve Titimas & $2=O L$ & JFL & $3-24-94$ \\
\hline N.0. STs V $\operatorname{sens}$ & Wistenens & Timson co & $3-2 y-9$ \\
\hline & & & \\
\hline & & & \\
\hline & & & \\
\hline & & & \\
\hline & & & \\
\hline & & & \\
\hline & & & \\
\hline & & & \\
\hline & & & \\
\hline & & & \\
\hline & & & \\
\hline & & & \\
\hline & & & \\
\hline & & & \\
\hline & . & & \\
\hline & & & \\
\hline & & & \\
\hline & & & \\
\hline & & & \\
\hline
\end{tabular}




\section{LEFPC PROJECT TRAINING RECORD}

SOP/LOA SIGNOFF

\begin{tabular}{|c|c|c|c|}
\hline \multicolumn{3}{|c|}{ SOP No. or LOA No.: $1661 B-001$ (LOA) } & $\begin{array}{l}\text { Revision } \\
\text { No.: * }\end{array}$ \\
\hline \multicolumn{4}{|c|}{ Title: Thermal Desorption Pilot Demonstration } \\
\hline \multicolumn{4}{|c|}{ I read and understood this procedure and will operate by it. } \\
\hline $\begin{array}{c}\text { Name } \\
\text { (Please Print) }\end{array}$ & Signature & Company & Date \\
\hline J. Fubrivio & & VRL TeeH Corp & $3-1-94$ \\
\hline S, Thumns & & VFC ted & $2-2-94$ \\
\hline R. ROSEBOROUAA & elrough & JFL TELH CORP. & $3 / 8 / 94$ \\
\hline J. Conuss: & & UFL TeEn CORP. & $3 / 8 / 94$ \\
\hline I. Rines & & UFL Tech COSP & $3-22-94$ \\
\hline D. noffma & 3 forture & VEL Tech Core & $3 / 22 / 54$ \\
\hline unefy L. Snimes & the & UfL TEeH COPP. & $3-2294$ \\
\hline N.V. STE & $\Gamma$ & $\begin{array}{r}\text { Jimsaxe } \frac{C_{0}}{I R} \\
\end{array}$ & $3-22-96$ \\
\hline J.D. Davis & Qlaws & ECOTEK/ARS & $3-23-94$ \\
\hline & & & \\
\hline & & . & \\
\hline & & & \\
\hline & & & \\
\hline & & & \\
\hline & & & \\
\hline & & & \\
\hline & & & \\
\hline & & & \\
\hline - & & & \\
\hline & & & \\
\hline & & & \\
\hline
\end{tabular}

* Revisions are not a pplicable to LOA's. If needed, addenda or special work instructione usill ho incod 
LEFPC PROJECT TRAINING RECORD

SOP/LOA SIGNOFF

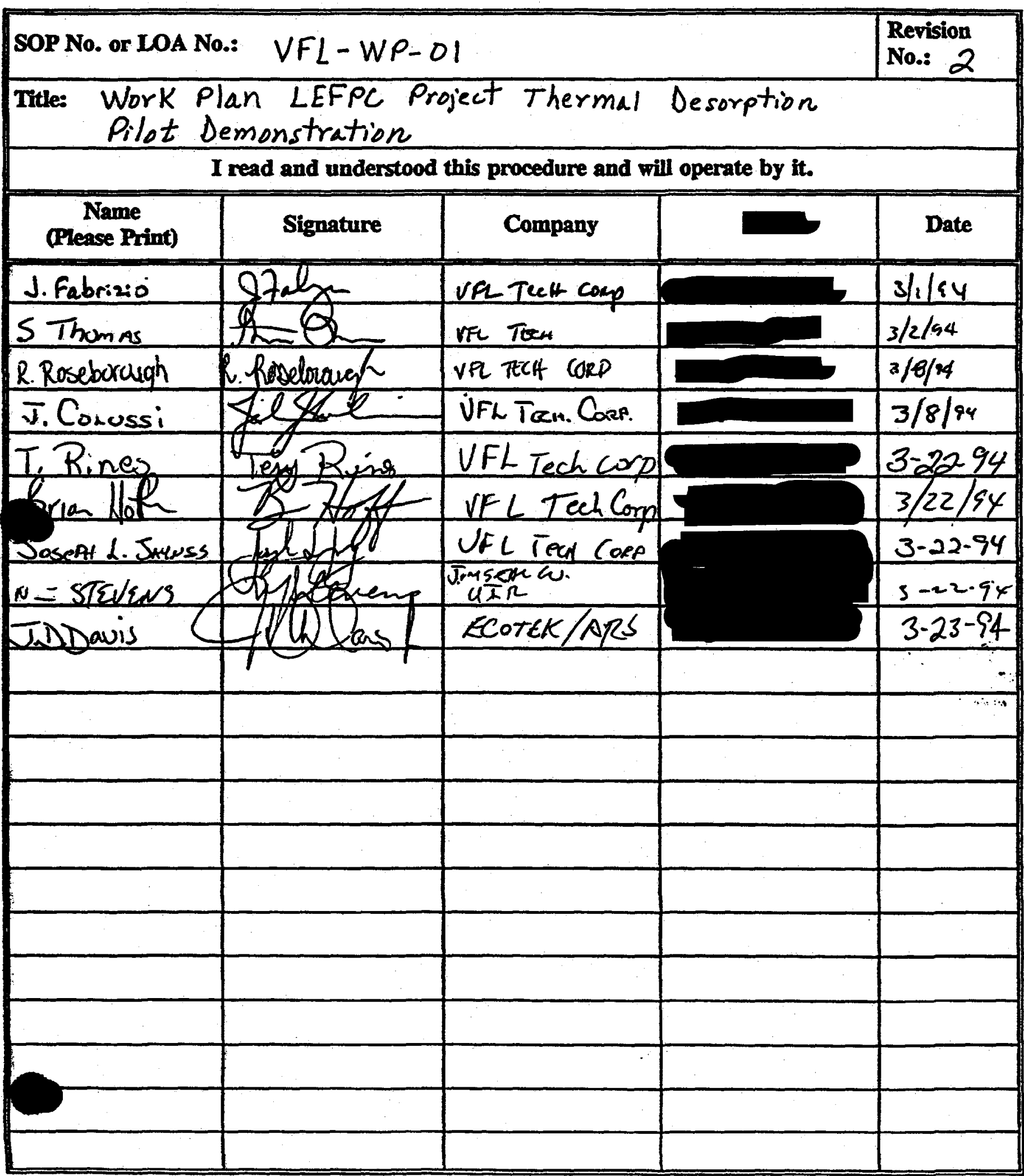


August 4, 1993

TO: $\quad$ Barry Griffith

FROM: Wade Tilson

\section{SUBJECT: SUPPLIER EVALUATIONS OF ANALYTICAL LABORATORIES}

Five analytical laboratories have been evaluated from a quality assurance perspective to perform analytical services in support of the UD\&D Project. The laboratories evaluated were as follows:

\begin{tabular}{||l|l|}
\hline \hline Halliburton NUS & \\
\hline EcoTek LSI & Pittsburgh, PA \\
\hline International Technology Corp., Inc. & Atlanta, GA \\
\hline Industrial and Environmental Analysts, Inc. & Research Triangle Park, NC \\
\hline Scientech, Inc. & Dallas, TX \\
\hline
\end{tabular}

The method used for evaluating the above laboratories was a review of their current quality records, i.e., Quality Assurance Program Manuals, previous QA audit reports, statements of qualification, where available, results of performance evaluation studies, and phone conversations with key personnel.

Attached are copies of the evaluation criteria used for each laboratory. Based on the evaluations conducted, all five (5) Laboratories are recommended for the approved vendor list (AVL). Upon award of a contract with either of the Laboratories, a performance based audit should be conducted early in the contract to verify adequate implementation of the written QA Programs.

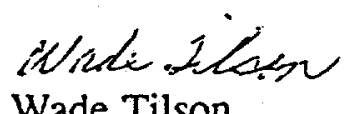

Wade Tilson

QC/TILSON:pll

Attachments

cc: M. A. Hopson

R. P. Slagle

R. Burckhardt

R. E. Kegley 
1. Does the laboratory have a Quality Assurance Plan?
a. ASME-NQA-1
b. DOE $5700.6 \mathrm{C}$
c. QAMS $005 / 80$ or CLP (EPA)
d. SW-846

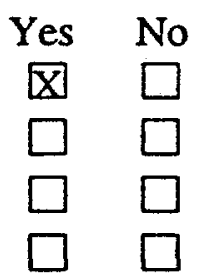

All laboratories submit copies of QA plans with quotes.

四

- Organization \& Responsibilities

- QA Level of Authority

- Separation of function

区

- Access to highest Management Level

网 $\square$

国

- Training of Personnel

- Documented Training Program

- Continuing Training Program

- Defined Disqualification Criteria

- Performance Standards

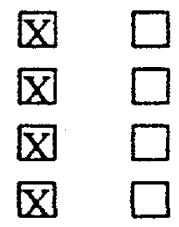

- Documented and Controlled Test Procedures

- Located in immediate work area

- Management approval of each procedure

四

- Document Control

四

- Procurement Document Control

- Quality Requirements

目

- Management Review

四

- Test Control

- Standards

- Software Controls

- Verification Program

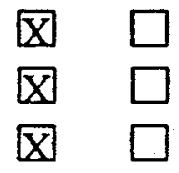


QUALTTY ASSURANCE EVALUATION CRITERIA FOR ANALYTICAL SERVICES REQUIRED FOR RFI PROGRAM

\section{(Continued)}

Control of Purchase Items

- Receipt Inspection Program

Yes No

四

Identification Controls

- Sample traceability (chain of custody)

四

- Equipment traceability

四

- Limited Life items

四

- Control of M\&TE

- Documented Calibration System, e.g.,

四

.. frequency, limits of acceptance, standards traceable to NIST

- Control of Non-Conforming Items and Data

- Documented Program

四

- Corrective Actions

- Methods of disposition

X

X

- Records and Data Management

- Identify quality records

- Record protection

四 $\square$

- Validation of records

口. 四*

四

- Audit Program

$\begin{array}{ll}\text { - } & \text { Frequency } \\ \text { - } & \text { Audit Type } \\ & \text { Qualification of Personnel }\end{array}$

$\begin{array}{ll}\text { 冈 } & \square \\ \text { 冈 } & \square \\ \text { 冈 } & \square * *\end{array}$

* Per LSI's response to Sarah Barron's audit report dated January 22, 1993, fire protection for files is included in the 1993 budget.

** Identified in written ciocument at site per Don Dihel telecon. 
QUALITY ASSURANCE EVALUATION CRITERIA FOR ANALYTICAL SERVICES REQUIRED FOR RFI PROGRAM

(Continued)

2. Audits by external agencies/contractors

Yes No

- EPA

网 $\square$

- DOE

$\square \quad \square$

$\square \quad \mathrm{X}$

Will give NFS copies of audits

四

3. CLP (EPA) Laboratory

- Organic

$\mathrm{X} \square$

- Inorganic

$\square \quad \square$

4. Conclusion:

Recommend the vendor be placed on Approved Vendor List (AVL)

Company: $\quad$ EcoTek Laboratory Services Incorporated

Company Location: Atlanta, GA

QC/LAB/CRITER04.RFI

Evaluated by

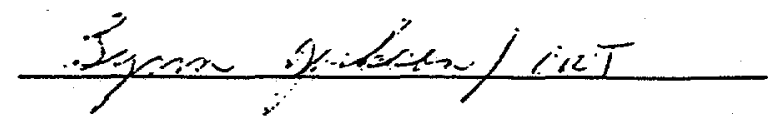

Date $2 / 2 y / i z$ 
February 9, 1994

TO: $\quad$ Bob Burckhardt

FROM: Wade Tilson

SUBJECT: Approved Vendor List

The Quality Assurance Program of Roy F. Weston, Inc. analytical laboratory, has been evaluated to determine if it will meet VFL' and NFS' needs for dif anal'yses on the LEFPC Project. The method used for evaluating the Weston Laboratory was a review of certain quality documents; i.e., Quality Assurance Program Plan (QAPP), Standard Practice 21-16-828D, dated July 17, 1990 (Organic Analysis Protocols Polychlorinated Dibenzo-P-Dioxins and Polychlorinated Dibenzofurans), and a recent audit performed on the Weston laboratory (E93-15 dated October 28, 1993). Assisting in this evaluation, from a technical perspective, were Mr. Randy Slagle and Mr. Doug Davis. Both people are highly qualified to evaluate Weston's technical capabilities. In addition, Mr. Davis served as an auditor/technical expert during the referenced audit.

Based on the results of this evaluation, it is recommended that Roy F. Weston, Inc. be added to your Approved Vendors List (AVL) for providing analytical services on environmental type samples, i.e., SW-846 protocols.

CWT:ckf

pc:
R. P. Slagle
M. A. Rush
J. D. Davis
B. L. Griffith

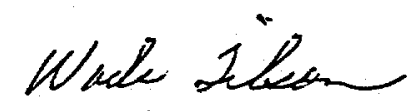

Wade Tilson 


\section{PROFESSIONAL PROFILE}

JOSEPH D. LOMIN, JR.

Fields of Competence

Trace analysis of dioxins/furans, volatile and semivolatile priority pollutants in various matrices using U.S. EPA CLP protocols.

\section{Experience Summary}

- Ten years experience in trace analys\&is of dioxins/furans, volatile and semivolatile priority pollutants in various matrices using U.S. EPA GC/MS methods.

- GC/MS analytical support for chemical research facility - method development and qualitative analysis.

- SFC method development.

\section{Credentials}

Studied Chemistry -- University of Delaware

Studied Optical Engineering -- University of Rochester

Employment History

1988-Present WESTON

1982-1988 Hercules, Inc.

1987 Computer Chemical Systems

Key Projects

Winfield Lock and Dam Site, Redhouse, WV, Law Environmental, dioxin/furan analyses. Detroit/Wayne County, Air Monitoring Program, PAH analyses. 


\begin{tabular}{|c|c|c|c|c|}
\hline . & $\begin{array}{c}\text { Jack R. Tuschall } \\
\text { Presideot } \\
\text { Chicf Execuive Officer } \\
\text { Marager, Lab. Operatioss } \\
19 \text { Years Experience } \\
\text { PhD Environmental } \\
\text { Engineering Sciesce } \\
\text { M.S. Environmental } \\
\text { Chemistry } \\
\text { B.S. Cherristy } \\
\cdots\end{array}$ & $\begin{array}{l}\text { Gregory Grandits } \\
\text { Menazer } \\
\text { Organiss Labonory } \\
\\
12 \text { Years Experiezce } \\
\text { B.S. Biology/Chemisury } \\
\text { A.A.S. Chenical } \\
\text {. Technology } \\
\text { A.A.S. Medical } \\
\text { Technology } \\
\text {.. }\end{array}$ & $\begin{array}{c}\text { Dolores Mick } \\
\text { Manager } \\
\text { Quality Assurance } \\
\text { 9 Years Experience } \\
\text { B.S: Business Admis. } \\
\text { (Junior Standing) } \\
\text { Accident Investigation } \\
\text { RAB Lead Assessor } \\
\text { ASQC Cerrified } \\
\text { Qualtiy Auditor } \\
\text { NQA-1 Lead Auditor }\end{array}$ & $\begin{array}{c}\text { Tara Pipes } \\
\text { Manager } \\
\text { Projecr Manegement } \\
\text { 8 Years Experience } \\
\text { M.S. Biochemistry } \\
\text { B.S. Anisal Science } \\
\text { Lead QA Auditor } \\
\\
\text { DOE-L } \\
\text { NRC-LR }\end{array}$ \\
\hline RADIOCIIEMISTRY & $y^{\prime}$ & $x_{2}$ & Fy & 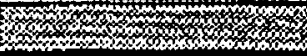 \\
\hline \multicolumn{5}{|l|}{ Mixed Fission Products } \\
\hline \multicolumn{5}{|l|}{ Activation Producls } \\
\hline \multicolumn{5}{|l|}{ Source Material } \\
\hline Trensuranies & & & XES & \\
\hline SNM & & & $\cdots$ & $\cdots$ \\
\hline \multicolumn{5}{|l|}{ Bio2ssay } \\
\hline Low Level Waste & & & YES & \\
\hline Mixed Waste & YES & & YES & \\
\hline \multicolumn{5}{|l|}{ 10CFR61 Analysis } \\
\hline Environmental & YES & & YES & \\
\hline \multicolumn{5}{|l|}{ Non-Destruecive Assay } \\
\hline \multicolumn{5}{|l|}{ Nuclear Spectroscopy } \\
\hline ORGANICS & 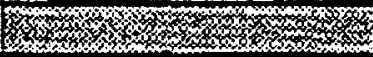 & $x_{z}$ & K & 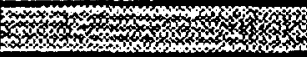 \\
\hline CLP & YES & YES & & YES \\
\hline SW-846 & YES & YES & & YES \\
\hline TCLP & YES & YES & & YES \\
\hline OCAMS & YES & YES & & \\
\hline FTIR & & & & YES \\
\hline OC.ECD & YES & YES & & YES \\
\hline GC-FID & YES & YES & & YES \\
\hline OC.PID & & YES & & VES \\
\hline GC.ELCD & & YES & & YES \\
\hline HPLC & YES & YES & & YES \\
\hline GPC & & YES & & YES \\
\hline DORGAMC & 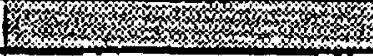 & 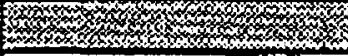 & W & 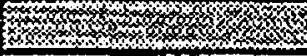 \\
\hline CLP & YES & & & \\
\hline SW-846 & YES & & YES & \\
\hline TCLP & YES & & . & \\
\hline ICP & YES & & & \\
\hline CVAA & YES & & & \\
\hline GFAA & YES & & YES & \\
\hline Flame AA & YES & & YES & \\
\hline IC & YES & & & \\
\hline Auro Analyzer & YES & & & \\
\hline TOC & YES & & & \\
\hline
\end{tabular}




\begin{tabular}{|c|c|c|c|c|}
\hline$\because$ & $\begin{array}{l}\text { Linda Hayhurst } \\
\text { Production Mansger } \\
\text { Radiounalyrical } \\
\text { Laboratory } \\
7 \text { Years Experience } \\
\text { B.S. Cherristy } \\
\\
\therefore \\
\therefore \\
\therefore\end{array}$ & $\begin{array}{l}\text { Thomas E. Connor } \\
\text { Regulanory \& } \\
\text { Sefety Spec. } \pi \\
\text { A.S. Enginecrits } \\
\text { Technologies } \\
\text { Over } 10,000 \text { brs. of } \\
\text { DOE \& DOD } \\
\text { Experience } \\
\text { Formal Rau.,Ind. Hygiede } \\
\text { Reguarrory Compliance } \\
\text { a }\end{array}$ & $\begin{array}{c}\text { Dorothy GIII } \\
\text { Nuclear Spec } \\
20 \text { Years Experience } \\
\text { BA Chem. } 2001 \\
\text { MS Analytical Chem. }\end{array}$ & $\begin{array}{l}\text { Mike Buchanan } \\
\text { Marager } \\
\text { Spacial Projecs } \\
\\
14 \text { Years Experience } \\
\text { M.S. Systems Mgrmi. } \\
\text { B.S. Chemical } \\
\text { Oceanography }\end{array}$ \\
\hline RADIOCHEMIISTRY & 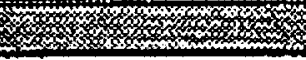 & 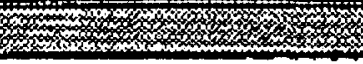 & 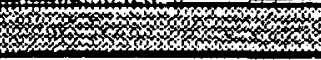 & 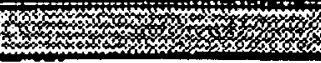 \\
\hline Mixed Fission Products & YES & YES & YES & \\
\hline Activation Products & YES & YES & YES & \\
\hline Source Material & YES & YES & YES & \\
\hline Transuranics & YES & YES & YES & \\
\hline SNM & & YES & YES & YES \\
\hline Bioassay & & YES & YES & \\
\hline Low Level Waste & YES & YES & YES & YES \\
\hline Mixed Waste & YES & YES & YES & YES \\
\hline 10CFR61 Andysis & YES & YES & YES & \\
\hline Environmencal & YES & YES & YES & YES \\
\hline Non-Destructive Assay & & YES & YES & \\
\hline Nuciear Speciroscopy & YES & YES & YES & \\
\hline RGANCS & $x_{x}$ & 而 & z & 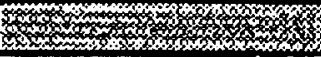 \\
\hline CLP & YES & YES & YES & YES \\
\hline $5 W-846$ & YES & & YES & YES \\
\hline TCLP & YES & YES & YES & YES \\
\hline OCMS & YES & & YES & YES \\
\hline FTIR & & & YES & YES \\
\hline GC-ECD & & & YES & YES \\
\hline GC-FID & YES & & YES & YES \\
\hline GC-PID & & & YES & YES \\
\hline GC-ELCD & & & YES & YES \\
\hline HPLC & & & YES & YES \\
\hline GPC & & & YES & YES \\
\hline INORGANC & 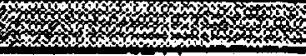 & Ascisis & 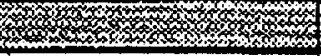 & 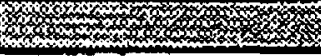 \\
\hline CLP & & YES & & YES \\
\hline sw-846 & & & YES & YES \\
\hline TCLP & & YES & YES & YES \\
\hline ICP & & & YES & YES \\
\hline CVAA & & & YES & YES \\
\hline GFAA & & & YES & YES \\
\hline Flame AA & & & YES & YES \\
\hline IC & & & YES & YES \\
\hline Xuto Anslyzer & & & YES & YES \\
\hline TOC & & YES & YES & YES \\
\hline
\end{tabular}




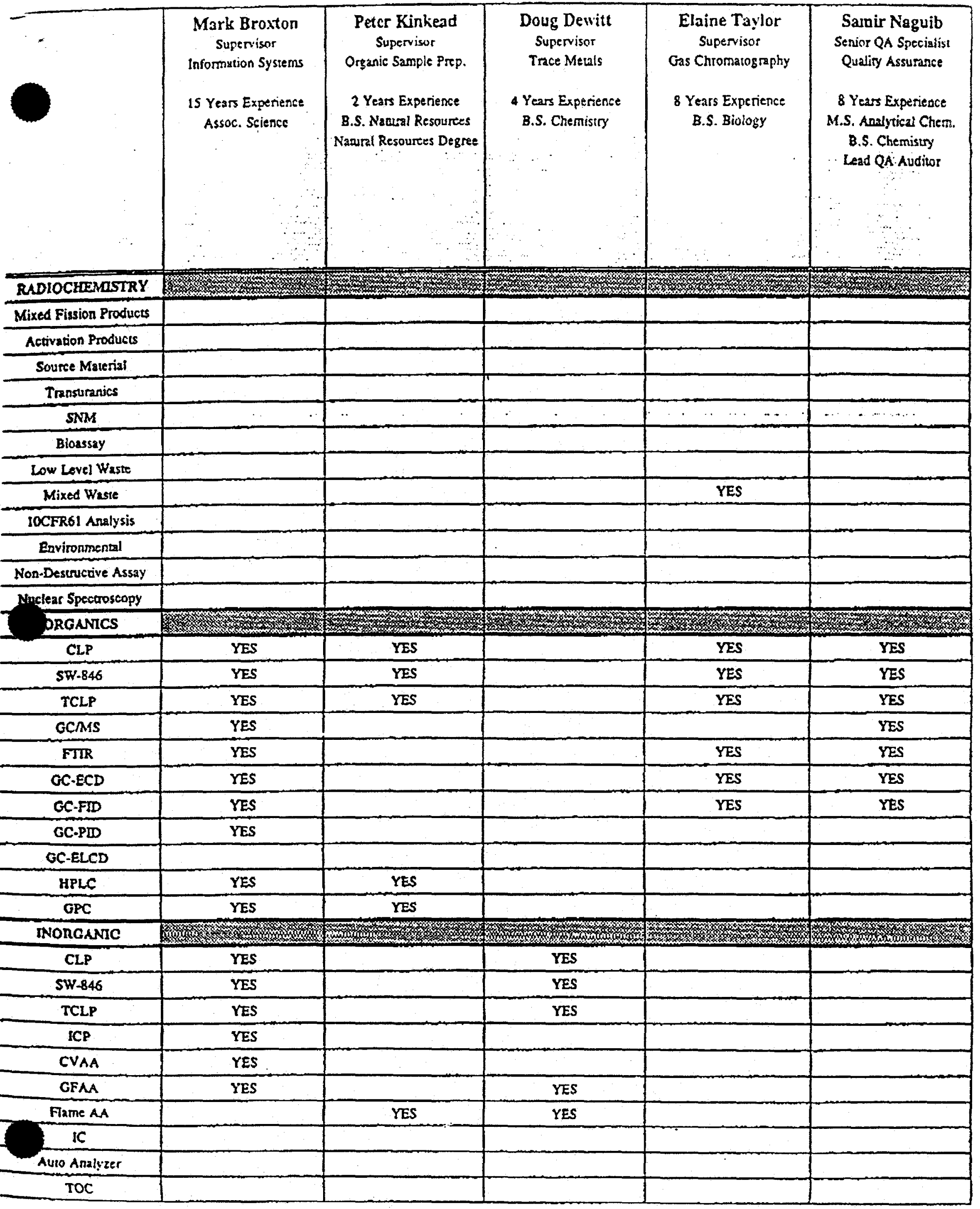




\begin{tabular}{|c|c|c|c|c|c|}
\hline 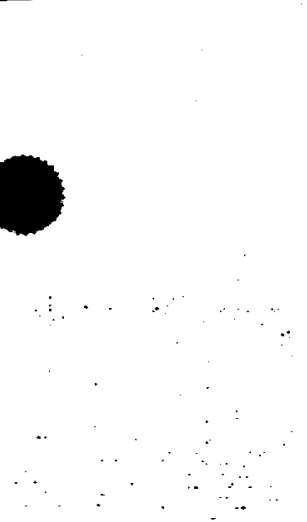 & 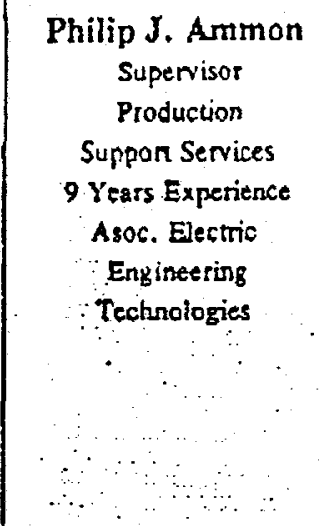 & $\begin{array}{l}\text { Chen Zhang } \\
\text { Analyst in } \\
\text { GCMS } \\
\text { to Years Experience } \\
\text { B.S. Chemisisy } \\
\end{array}$ & $\begin{array}{c}\text { Linda Lu } \\
\text { Aralyst id } \\
\text { OC/MS } \\
9 \text { Years Experience } \\
\text { B.S. Chemistry } \\
\text { M.S. Chemistry } \\
\therefore\end{array}$ & $\begin{array}{c}\text { Bing Xu } \\
\text { Analyst IfI } \\
\text { GC/MS } \\
7 \text { Years Experience } \\
\text { B.S. Chemistry }\end{array}$ & 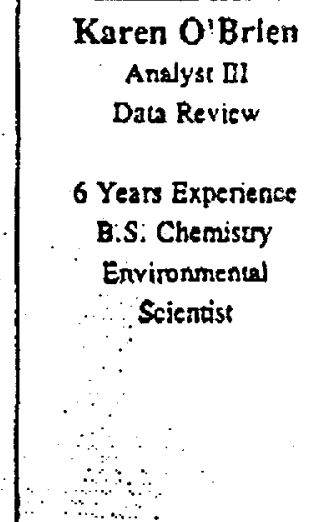 \\
\hline RADIOCHEMISTKY & I & < & 16 & 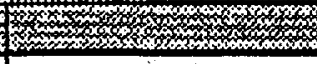 & $x_{2}$ \\
\hline Mixed Fission Products & YES & & & & \\
\hline Activarion Products & YES & & & & \\
\hline Sourec Material & YES & & & & \\
\hline Trnnsumnics & YES & & & & \\
\hline SNM & YES & $\dot{0}$ & . & $\dot{5}$ & $\ldots \ldots \ldots$ \\
\hline \multicolumn{6}{|l|}{ Bionsssy } \\
\hline Low Level Waste & YES & & & & \\
\hline Mixed Whase & YES & YES & YES & YES & \\
\hline \multicolumn{6}{|l|}{ 10CFR61 Analysis } \\
\hline Envisonmental & YES & & & & YES \\
\hline \multicolumn{6}{|l|}{ Non-Destuctive Assay } \\
\hline Muclear Spectroscopy & YES & & & & \\
\hline ORGAMCS & $y_{2}$ & 西 & f & S & 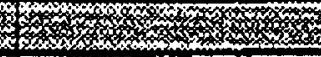 \\
\hline CLP & & YES & YES & YES & \\
\hline SW-846 & & YES & YES & YES & YES \\
\hline TCLP & & YES & YES & YES & YES \\
\hline OCMS & YES & YES & YES & YES & YES \\
\hline FTIR & YES & YES & YES & & \\
\hline GC-ECD & YES & YES & YES & & \\
\hline GC-FID & YES & YES & YES & YES & YES \\
\hline GC.PID & YES & YES & YES & YES & YES \\
\hline GC.ELCD & YES & YES & YES & & \\
\hline HPLC & YES & YES & YES & YES & \\
\hline GPC & YES & YES & & YES & \\
\hline INORGANC & $V_{2}$ & 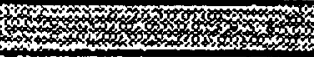 & $\frac{1}{2} \times 1 \%$ & 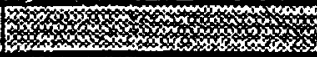 & 1 \\
\hline \multicolumn{6}{|l|}{ CLP } \\
\hline SW-846 & & & & & YES \\
\hline TCLP & & & & & YES \\
\hline $\mathbf{I C P}$ & YES & YES & & & \\
\hline CVAA & YES & YES & & & \\
\hline GFAA & YES & YES & YES & & \\
\hline Flame AA & YES & YES & YES & YES & YES \\
\hline IC & YES & YES & & YES & \\
\hline Auto Analyzet & YES & & YES & & \\
\hline TOC & YES & YES & & & YES \\
\hline
\end{tabular}




\begin{tabular}{|c|c|c|c|c|c|}
\hline$\because \vdots$ & $\begin{array}{c}\text { Joe Rebstock } \\
\text { Analyst III } \\
\text { Data Review } \\
27 \text { Years Experience } \\
\qquad \quad \\
\text { Top Secret DOD } \\
\text { Secret DIS }\end{array}$ & $\begin{array}{c}\text { Mei Liang } \\
\text { Analyst Il } \\
\text { GCiMS } \\
22 \text { Years Experiedce } \\
\text { B.S. Chemistry }\end{array}$ & $\begin{array}{c}\text { Tim Welch } \\
\text { Asalysi II } \\
\text { GCMMS } \\
4 \text { Years Experience } \\
\text { B.S. Zoology } \\
\text { DOE-L } \\
\text { NRC-L }\end{array}$ & $\begin{array}{c}\text { Bruce MacAllister } \\
\text { Analyst Il } \\
\text { Trace Metais } \\
\text { 8 Years Expetiesce } \\
\text { B.S. Biology } \\
\end{array}$ & $\begin{array}{l}\text { Doug Anderson } \\
\text { Analyst il } \\
\text { Organic Sample Prep. } \\
\text { 1.5 Years Experience } \\
\text { B.S. Molecular Biology } \\
\vdots \\
\therefore \\
\therefore\end{array}$ \\
\hline RADIOCIIEMISTRY & $y_{1}$ & ( & 2) & 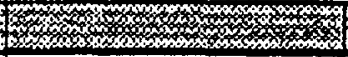 & 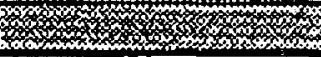 \\
\hline Mixed Fission Products & YES & & & & \\
\hline Acrivation Products & YES & & & & \\
\hline Source Material & YES & & & & \\
\hline Transuranics & YES & & & & \\
\hline SNM & YES & & & & \\
\hline \multicolumn{6}{|l|}{ Bioassay } \\
\hline Low Level Weste & YES & & & & \\
\hline Mixed Weste & YES & YES & YES & & \\
\hline \multicolumn{6}{|l|}{ 10CFR6: Anslysis } \\
\hline Environmental & YES & & & & YES \\
\hline Non-Destruetive Assay & YES & & & & \\
\hline Nevelear Specuroscopy & YES & & & & \\
\hline ORGANICS & $\gamma_{x}$ & 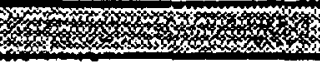 & 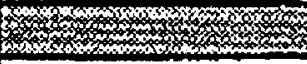 & 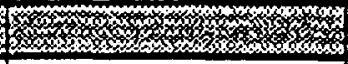 & $\gamma_{2}$ \\
\hline CLP & & YES & YES & & YES \\
\hline SW-846 & & YES & YES & & YES \\
\hline RCLP & & YES & YES & & YES \\
\hline GCMS & & & YES & & \\
\hline FTIR & & YES & & & YES \\
\hline GC-ECD & & YES & YES & & \\
\hline GC-FID & & YES & YES & & YES \\
\hline GC.PID & & YES & YES & & YES \\
\hline GC-ELCD & & YES & YES & & YeS \\
\hline \multicolumn{6}{|l|}{ HPLC } \\
\hline \multicolumn{6}{|l|}{ GPC } \\
\hline NORGANIC & 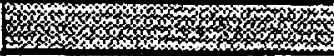 & 2 & $y_{3}^{\prime}$ & (x) & 6 \\
\hline CLP & & YES & & YES & \\
\hline SW.846 & & VES & & YES & \\
\hline TCLP & & YES & & YES & \\
\hline ICP & YES & YES & & YES & \\
\hline CVAA & & YES & & YES & \\
\hline GFAA & YES & YES & & YES & \\
\hline Flame AA & YES & & & YES & $\therefore$ \\
\hline \multicolumn{6}{|l|}{ IC } \\
\hline Auto Analyzer & & & YES & & YES \\
\hline TOC & & & & & \\
\hline
\end{tabular}




\begin{tabular}{|c|c|c|}
\hline 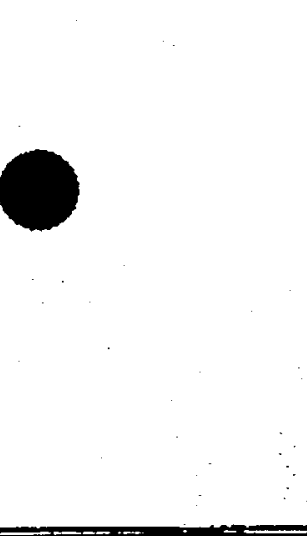 & $\begin{array}{c}\text { Leslie Papa } \\
\text { Analyss U } \\
\text { Truse Metals } \\
\text { S Years Expenenze } \\
\text { B.S. Biology } \\
\\
\end{array}$ & $\begin{array}{l}\text { Joel Steptens } \\
\text { Section Supervisur } \\
\text { Genera! Chemiscry } \\
\text { SYcars Experience } \\
\text { B.S. Biology } \\
\text { DOE Securiry } \\
\end{array}$ \\
\hline RADIOCHEMUSTRY & 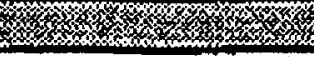 & $y_{x}$ \\
\hline Mixed Fission Products & & YES \\
\hline Activetion Producs & & YES \\
\hline \multicolumn{3}{|l|}{ Source Material } \\
\hline \multicolumn{3}{|l|}{ Trensuranics } \\
\hline \multicolumn{3}{|l|}{ SNM } \\
\hline \multicolumn{3}{|l|}{ Biozssay } \\
\hline Low Level Waste & & YES \\
\hline Mixed Waste & & YES \\
\hline \multicolumn{3}{|l|}{ IOCPR61 Analysis } \\
\hline Environmeneal & & YES \\
\hline \multicolumn{3}{|l|}{ Non-Desurucrive Assay } \\
\hline \multicolumn{3}{|l|}{ Nuclear Spectroscopy } \\
\hline \multicolumn{3}{|l|}{ RCANICS } \\
\hline CLP & YES & \\
\hline SW-846 & YES & \\
\hline \multicolumn{3}{|l|}{ TCLP } \\
\hline \multicolumn{3}{|l|}{ GCMS } \\
\hline \multicolumn{3}{|l|}{ PTIR } \\
\hline GC-ECD & YES & \\
\hline OC-RID & YES & \\
\hline \multicolumn{3}{|l|}{ GC-PID } \\
\hline \multicolumn{3}{|l|}{ GC-ELCD } \\
\hline \multicolumn{3}{|l|}{ KPLC } \\
\hline \multicolumn{3}{|l|}{ GPC } \\
\hline DNORGANIC & 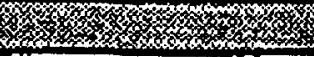 & $\gamma_{3}$ \\
\hline CLP & YES & YES \\
\hline SW-846 & YES & YES \\
\hline TCLP & YES & \\
\hline ICP & YES & \\
\hline CVAA & YES & \\
\hline GFAA & YES & \\
\hline Flame $A$ A & YES & \\
\hline IC & & YES \\
\hline Auro Analyzer & & \\
\hline TOC & & YES \\
\hline
\end{tabular}


APPENDIX III

SOILS CHARACTERIZATION 


\section{VFL TECHNOLOGY CORPORATION}

42 LLOYD AVENUE - MALVERN, PA 19355 - (215) 296-2233 • FAX (215) 296-9545

\section{January 31, 1994}

\section{Ms. Lisa L. Cobb}

Purchasing Department

Martin Marietta Energy Systems, Inc.

P.O. Box 2002

Oak Ridge, TN 37831-6501

RE: Lower East Fork Poplar Creek

Subcontract No. 82Y-05556

VFL Project No. C-2814

Soil Characterization Data (Rev 01)

Dear Ms. Cobb:

Enclosed herein please find six (6) copies of the Soil Characterization Data (Rev 01) Tables $\# 1$ and $\# 2$ for the above referenced project.

Kindly review the enclosed and advise us of your comments.

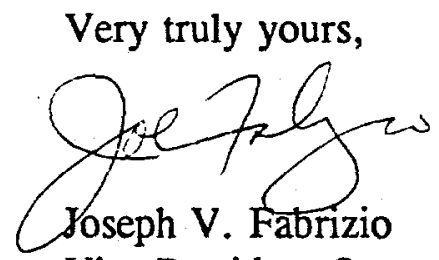

Vice President Operations

\section{$\mathrm{JVF} / \mathrm{plh}$}

Enclosure

cc: Mr. F. S. Thomas - VFL 

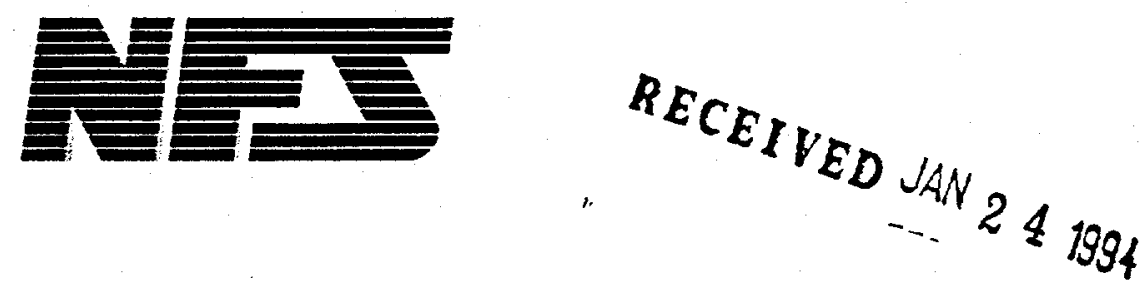

Nuclear Fuel Services, Inc.

50G-94-0010

DC-94-008

SAL-04-93

SO 1661B

January 19, 1994

Mr. Joe Fabrizio, Project Manager

VFL Technology Corporation

42 Lloyd Avenue

Malvern, PA 19355

Subject: $\quad$ LEFPC Soil Characterization Data - Revision 1

Dear Mr. Fabrizio:

Attached are a corrected memo and Tables 1 and 2 that provide the soil characterization data from the analysis of the duplicate set of LEFPC samples sent to NFS on November 19, 1993. This is a deliverable to MMES under Task 3. Please discard the original letter DC-94-006 as there was a calculation error in the confidence intervals (CI). A copy is also being faxed to you today.

Sincerely,

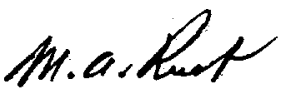

R. D. Clark

Sales \& Contract Administrator

Idsf

Attachment
cc: CE Athon
SM Duncan
CW Tilson

DE Coulter

JW Pugh

DJ Cronshaw

JD Davis

MA Rush

RP Slagle 
January 19, 1994

TO: $\quad$ M.A. Rush

FROM: J.D. Davis

\section{SUBJECT: LEFPC Soil Characterization Data - Revision 1}

NOTE: Revision 1 supersedes the original memo JDD-94-001, dated 1/14/94. Please discard the original as there was a calculation error in the confidence interval (CI).

This memo provides the soil characterization data from the analysis of the duplicate set of LEFPC samples sent to NFS on November 19, 1993. Each of the six soil samples was analyzed for total mercury, arsenic, cadmium, PCBs, uranium, VOCs, and PAHs. Analyses were performed at EcoTek, LSI in Atlanta, Georgia.

Table 1 is a summary of the LEFPC soil characterization data from EcoTek, LSI. An $80 \%$ confidence interval $(\mathrm{CI})$ was calculated for each analyte according to SW-846 guidance. Mercury, the primary analyte of interest, had an average concentration of $475 \mathrm{ppm}$ for the five sample locations. The $80 \% \mathrm{CI}$ around the mercury concentration was $\pm 78 \mathrm{ppm}$. The QC data was acceptable for all analyses except total cadmium. The matrix spike recovery for cadmium analyses was only $25 \%$ (QC limits are 75 to $125 \%$ ). Since the matrix spike recovery for the method blank was acceptable, the above result was judged to be caused by matrix interference. The cadmium result reported in Table 1 should be considered as an estimated value.

Table 2 is a comparison of LEFPC soil characterization data from EcoTek, LSI and MMES. The MMES data used for the comparison is the preliminary data given to NFS at the December 16, 1993 review meeting in Oak Ridge. The mercury and uranium concentrations reported by EcoTek, LSI were nearly twice as high as the values reported by MMES. Both sets of analyses confirm that the soil is contaminated with mercury at levels such that a meaningful thermal desorption study can be performed. Analyzing the soil as it is input into treatment (according to the Sampling and Analysis Plan, NFS-M-055) will provide baseline information concerning the actual concentration of mercury in the untreated soil.

lidsf

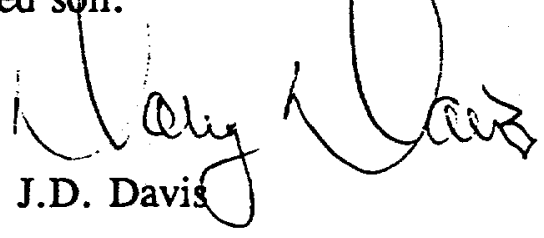

cc: DJ Cronshaw CE Athon
CW Tilson

RD Clark
RP Slagle SM Duncan
JW Pugh

DE Coulter 
Table 1 - Summary of LEFPC Soil Characterization Data from EcoTek, LSI

\begin{tabular}{|c|c|c|c|c|c|c|c|c|c|}
\hline & & \multicolumn{5}{|c|}{ Sample No. } & \multirow{2}{*}{$\begin{array}{c}\text { Field } \\
\text { Duplicate }\end{array}$} & \multirow{2}{*}{$\begin{array}{c}\text { Average } \\
\text { Concentration (1) }\end{array}$} & \multirow{2}{*}{$\begin{array}{l}\text { Confidenoe } \\
\text { Interval (2) }\end{array}$} \\
\hline & & $23 \sqrt{24}$ & 9/11 & $4 / 14$ & $1 / 3$ & $19 / 27$ & & & \\
\hline Metals & $\begin{array}{l}\text { Mercury, ppm } \\
\text { Arsenic, ppm } \\
\text { Cadmium, ppm } \\
\text { Uranium, ppm }\end{array}$ & $\begin{array}{r}463 \\
11 \\
10 \\
35\end{array}$ & $\begin{array}{r}303 \\
10 \\
11 \\
30\end{array}$ & $\begin{array}{r}604 \\
11 \\
10 \\
33\end{array}$ & $\begin{array}{r}548 \\
7 \\
7 \\
25\end{array}$ & $\begin{array}{r}458 \\
10 \\
9 \\
29\end{array}$ & $\begin{array}{r}1040 \\
11 \\
8 \\
28\end{array}$ & $\begin{array}{r}475 \\
10 \\
9 \\
30\end{array}$ & $\begin{array}{r}553 \\
11 \\
10 \\
33\end{array}$ \\
\hline PCBs & Aroclor $-1260, \mathrm{ppb}$ & 2040 & 2280 & 1630 & 1480 & 1990 & 2110 & 1884 & 2106 \\
\hline VOCs & Carbon Tetrachloride, ppb & 8 & 8 & 9 & 6 & 4 & 8 & 7 & 8 \\
\hline PAHs & $\begin{array}{c}\text { Fluoranthene, } \mathrm{ppb} \\
\text { Pyrene, } \mathrm{ppb} \\
\text { Benzo(a)anthracene, } \mathrm{ppb} \\
\text { Chrysene, } \mathrm{ppb} \\
\text { Benzo(b)fluorcanthene, } \mathrm{ppb} \\
\text { Benzo(a)pyrene, } \mathrm{ppb} \\
\text { Phenanthrene, } \mathrm{ppb} \\
\text { Naphthalene, } \mathrm{ppb} \\
\text { Acetonaphthene, } \mathrm{ppb} \\
\text { Fluorene, ppb } \\
\text { Anthracene, ppb } \\
\text { Ideno(1,2,3-cd)pyrene, ppb } \\
\text { Benzo(g,h,i)perylene, ppb }\end{array}$ & $\begin{array}{r}970 \\
1400 \\
550 \\
550 \\
830 \\
490 \\
610 \\
\mathrm{BQL} \\
\mathrm{BQL} \\
\mathrm{BQL} \\
\mathrm{BQL} \\
\mathrm{BQL} \\
\mathrm{BQL}\end{array}$ & $\begin{array}{r}1100 \\
1900 \\
690 \\
790 \\
1200 \\
710 \\
690 \\
\mathrm{BQL} \\
\mathrm{BQL} \\
\mathrm{BQL} \\
\mathrm{BQL} \\
\mathrm{BQL} \\
\mathrm{BOL}\end{array}$ & $\begin{array}{r}2600 \\
3500 \\
1500 \\
1200 \\
1900 \\
950 \\
2600 \\
500 \\
430 \\
550 \\
600 \\
\mathrm{BQL} \\
\mathrm{BQL}\end{array}$ & $\begin{array}{r}1200 \\
1700 \\
710 \\
770 \\
1100 \\
650 \\
690 \\
\mathrm{BQL} \\
\mathrm{BQL} \\
\mathrm{BQL} \\
\mathrm{BQL} \\
560 \\
\mathrm{BQL}\end{array}$ & $\begin{array}{r}1500 \\
2300 \\
910 \\
920 \\
1400 \\
860 \\
860 \\
\text { BQL } \\
\text { BQL } \\
\text { BQL } \\
\text { BQL } \\
690 \\
680\end{array}$ & $\begin{array}{c}1100 \\
1900 \\
630 \\
770 \\
1000 \\
680 \\
650 \\
\mathrm{BQL} \\
\mathrm{BQL} \\
\mathrm{BQL} \\
\mathrm{BQL} \\
\mathrm{BQL} \\
\mathrm{BQL}\end{array}$ & $\begin{array}{r}1474 \\
2160 \\
872 \\
846 \\
1286 \\
732 \\
1090 \\
100 \\
86 \\
110 \\
120 \\
250 \\
136\end{array}$ & $\begin{array}{r}1926 \\
2720 \\
1128 \\
1009 \\
1560 \\
855 \\
1672 \\
253 \\
218 \\
279 \\
304 \\
487 \\
344\end{array}$ \\
\hline
\end{tabular}

(1) Average concentration does not include Field Duplicate.

(2) Upper limit of the $80 \% \mathrm{Cl}$ as defined in SW-846. 
JDD-94-002

DEV

Page 3

Table 2 - Comparison of LEFPC Soil Characterization Data from EcoTek, LSI and MMES

\begin{tabular}{|c|c|c|}
\hline $\begin{array}{c}\text { Analyte } \\
\text { (total) }\end{array}$ & $\begin{array}{c}\text { EcoTek, LSI, } \\
\text { avg ppm }\end{array}$ & $\begin{array}{c}\text { MMES, } \\
\text { avg ppm }\end{array}$ \\
\hline Mercury & 475 & 254 \\
Uranium & 30 & 17 \\
Arsenic & 10 & $<20$ \\
Cadmium & 9 & 8 \\
PCBs & 1.88 & 0.50 \\
\hline
\end{tabular}




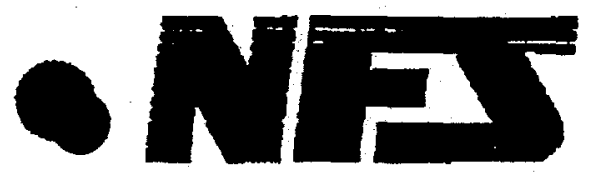

Nuctear Fuel Sentces, inc. P.O. BOX 337, MS 123 Envin. IN 37650

(615) $743-9141$

50G-94-0006

DC-94-006

SAL-04-93

SO $1661 B$

January 14, 1994

Mr. Joe Fabrizio, Project Manager

VFL Technology Corporation

42 Lloyd Avenue

Malvern, PA 19355

Subject: LEFPC Soil Characterization Data

Dear Mr. Fabrizio:

Attached is a memo providing the soil characterization data from the analysis of the duplicate set of LEFPC samples sent to NFS on November 19, 1993. This is a deliverable to MMES under Task 3. A copy is also being faxed to you today.

Sincerely,

M.G. Runh

R. D. Clark

Sales \& Contract Administrator

Idsf

Attachment
cc: CE Athon
DE Coulter
DJ Cronshaw
JD Davis
SM Duncan
JW Pugh
MA Rush
RP Slagle
CW Tilson 
January 14, 1994

TO: M.A. Rush

FROM: J.D. Davis

SUBJECT: LEFPC Soil Characterization Data

This memo provides the soil characterization data from the analysis of the duplicate set of LEFPC samples sent to NFS on November 19, 1993. Each of the six soil samples was analyzed for total mercury, arsenic, cadmium, PCBs, uranium, VOCs, and PAHs. Analyses were performed at EcoTek, LSI in Attanta, Georgia.

Table 1 is a summary of the LEFPC soil characterization data from EcoTek, LSI. An 80\% confidence interval $(\mathrm{Cl})$ was calculated for each analyte according to SW-846 guidance. Mercury, the primary analyte of interest, had an average concentration of $475 \mathrm{ppm}$ for the five sample locations. The $80 \% \mathrm{Cl}$ around the mercury concentration was $\pm 59 \mathrm{ppm}$. The $Q C$ data was acceptable for all analyses except total cadmium. The matrix spike recovery for cadmium analyses was only $25 \%$ (QC limits are 75 to $125 \%$ ). Since the matrix spike recovery for the method blank was acceptable, the above result was judged to be caused by matrix interference. The cadmium result reported in Table 1 should be considered as an estimated value.

Table 2 is a comparison of LEFPC soil characterization data from EcoTek, LSI and MMES. The MMES data used for the comparison is the preliminary data given to NFS at the December 16, 1993 review mecting in Oak Ridge. The mercury and uranium concentrations reported by EcoTek, LSI were nearly twice as high as the values reported by MMES. Both sets of analyses confirm that the soil is contaminated with mercury at levels such that a meaningful thermal desorption study can be performed. Analyzing the soil as it is input into treatment (according to the Sampling and Analysis Plan, NFS-M-055) will provide baseline information concerning the actual concentration of mercury in the untreated sof.

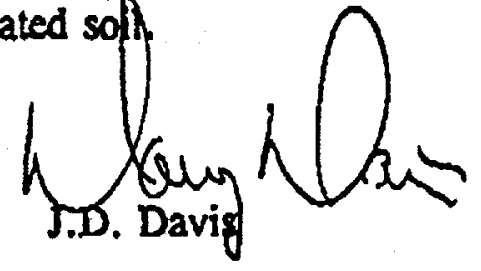

/dsf
cc: DJ Cronshaw
CW Tilson
CE Athon
RD Clark
RP Slagle
JW Pugh
SM Duncan
DE Coulter 
Table 1 - Summary of LEFPC Soil Characterization Data from EcoTek, LSI

\begin{tabular}{|c|c|c|c|c|c|c|c|c|c|}
\hline & & \multicolumn{5}{|c|}{ Sample No, } & \multirow{2}{*}{$\begin{array}{c}\text { Ficld } \\
\text { Duplicate } \\
\end{array}$} & \multirow{2}{*}{$\begin{array}{c}\text { Average } \\
\text { Concentration (1) }\end{array}$} & \multirow{2}{*}{$\begin{array}{l}\text { Confidente } \\
\text { Interval (2) }\end{array}$} \\
\hline & & 2324 & $9 / 11$ & $4 / 14$ & 13 & $19 / 27$ & & & \\
\hline Metals & $\begin{array}{l}\text { Mercury, ppm } \\
\text { Arsenic, ppm } \\
\text { Cadmium, ppm } \\
\text { Urarium, ppm }\end{array}$ & $\begin{array}{r}463 \\
11 \\
10 \\
35\end{array}$ & $\begin{array}{r}303 \\
10 \\
11 \\
30\end{array}$ & $\begin{array}{r}604 \\
11 \\
10 \\
33\end{array}$ & $\begin{array}{r}548 \\
7 \\
7 \\
25\end{array}$ & $\begin{array}{r}458 \\
10 \\
9 \\
29\end{array}$ & $\begin{array}{r}1040 \\
11 \\
8 \\
28\end{array}$ & $\begin{array}{r}475 \\
10 \\
9 \\
30\end{array}$ & $\begin{array}{r}534 \\
11 \\
10 \\
34\end{array}$ \\
\hline PCBs. & Aroclor - 1260, ppb & 2040 & 2080 & 1630 & 1480 & 1990 & 2110 & 184 & 2105 \\
\hline Vocs & Carbon Tetrachloride, ppb & 8 & 8 & 9 & 6 & 4 & 8 & 7 & 8 \\
\hline PAHs & 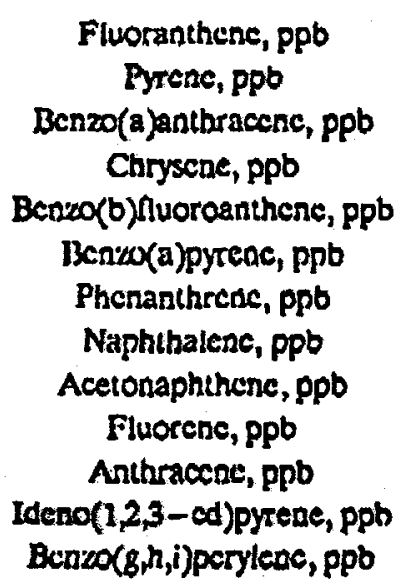 & $\begin{array}{r}970 \\
1400 \\
550 \\
550 \\
830 \\
400 \\
610 \\
B O L \\
B O L \\
B O L \\
B O L \\
B O L \\
B O L\end{array}$ & $\begin{array}{r}1100 \\
1900 \\
600 \\
790 \\
1200 \\
710 \\
690 \\
B Q L \\
B Q L \\
B Q L \\
B O L \\
B Q L \\
B Q L\end{array}$ & $\begin{array}{r}2600 \\
3500 \\
1500 \\
1200 \\
1900 \\
950 \\
2600 \\
500 \\
430 \\
550 \\
600 \\
B Q L \\
B Q L\end{array}$ & $\begin{array}{r}1200 \\
1700 \\
710 \\
770 \\
1100 \\
650 \\
690 \\
B O t \\
B Q L \\
B O L \\
B O L \\
5 C 0 \\
B O L\end{array}$ & $\begin{array}{r}1500 \\
2300 \\
910 \\
920 \\
1400 \\
8 \times 0 \\
800 \\
B O L . \\
B Q L \\
B Q L \\
B O L \\
690 \\
6 \$ 0\end{array}$ & $\begin{array}{r}1100 \\
1900 \\
630 \\
770 \\
1000 \\
680 \\
650 \\
B O L . \\
B Q L \\
\text { BQL } \\
\text { BOL } \\
13 O L \\
\text { BOL }\end{array}$ & $\begin{array}{r}1474 \\
2160 \\
872 \\
846 \\
1286 \\
732 \\
1090 \\
100 \\
86 \\
110 \\
120 \\
250 \\
136\end{array}$ & $\begin{array}{r}1701 \\
2469 \\
1004 \\
955 \\
1457 \\
823 \\
1327 \\
155 \\
133 \\
171 \\
186 \\
338 \\
211\end{array}$ \\
\hline
\end{tabular}

(1) Average concentration does not loclude Fiekd Duplicate.

(2) Upper limit of the $80 \% \mathrm{Cl}$ as defined in SW-84G 
APPENDIX IV

PILOT SCALE TRIAL RUNS

Test Results

Sample Logs 


\section{ARS/ECOTEK}

\section{LEFPC SCOPING TEST RESULTS}

\begin{tabular}{||l|l|l||}
\hline \multicolumn{1}{|c|}{ SAMPLE } & \multicolumn{1}{|c|}{ SAMPLE NUMBER } & MERCURY RESULTS (PFM) \\
\hline FEED & $1661 \mathrm{~B}-042994-$ F009 & 345 \\
\hline FEED & $1661 \mathrm{~B}-042994-$ F010 & 344 \\
\hline FEED & $1661 \mathrm{~B}-042994-$ F011 & 375 \\
\hline FEED & $1661 \mathrm{~B}-042994-$ F012 & 256 \\
\hline TREATED SOIL & $1661 \mathrm{~B}-042994-$ P013 & 26 \\
\hline TREATED SOIL & $1661 \mathrm{~B}-042994-$ P014 & 23 \\
\hline TREATED SOIL & $1661 \mathrm{~B}-042994-$ P015 & 29 \\
\hline TREATED SOIL & $1661 \mathrm{~B}-042994-$ P016 & 20 \\
\hline $\begin{array}{l}\text { SPIKE OF 1661B-042994- } \\
\text { FO09 }\end{array}$ & $1661 \mathrm{~B}-042994-$ F009-SPIKE & 868 SPIKE RECOVERY \\
\hline $\begin{array}{l}\text { SPIKE OF 1661B-042994- } \\
\text { P013 }\end{array}$ & $1661 \mathrm{~B}-042994-$ P013-SPIKE & $88 \%$ SPIKE RECOVERY \\
\hline QC CHECK STANDARD & 1.0 PPM MERCURY STD & 1.13 (1138 RECOVERY) \\
\hline BLANK & BLANK & 0.01 \\
\hline
\end{tabular}

SEE PRQJECT LAB NOT SBOOK FOR CALCULATIONS AND INDIVIDUAL RESULTS

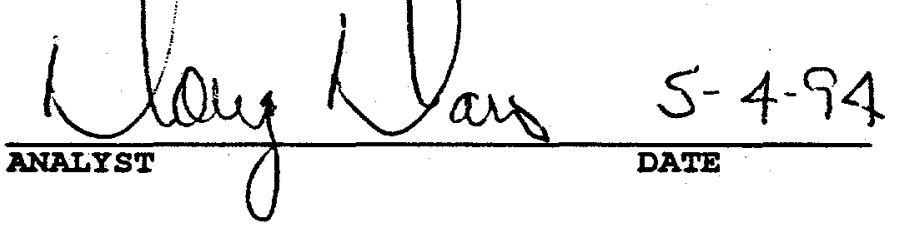




\section{ARS/ECOTEK}

\section{LEFPC SCOPING TEST RESULTS}

\begin{tabular}{||l|l|l||}
\hline \multicolumn{1}{|c|}{ SAMPLE } & \multicolumn{1}{|c|}{ SAMPLE NUMBER } & MERCURY RESULTS (PPM) \\
\hline FEED & F042594-45R & 294 \\
\hline FEED & F042594-75R & 203 \\
\hline TREATED SOIL & P042594-150 & 28 \\
\hline CONDENSER SOLIDS & 1661 B-008R & 212 \\
\hline TREATED SOIL FROM PIPE & $050294-019$ & 89 \\
\hline COLD TRAP & $1661 B-017$ & 0.04 \\
\hline CONDENSER SOLIDS & $1661 B-018$ & 321 \\
\hline CONDENSER LIQUID & 1661 B-018 & 0.29 \\
\hline TREATED COND. LIQUID & $1661 B-020$ & 0.41 \\
\hline SPIKE OF FO42594-45 & F042594-45 SPIKE & 928 SPIKE RECOVERY \\
\hline SPIKE OF 1661B-020 & $1661 B-020$ SPIKE & 1178 SPIKE RECOVERY \\
\hline $\begin{array}{l}\text { QC CHECK STANDARD- } \\
\text { SOLIDS }\end{array}$ & 1.0 PPM MERCURY STD & 1.02 (1028 RECOVERY) \\
\hline $\begin{array}{l}\text { QC CHECK STANDARD- } \\
\text { LIQUID }\end{array}$ & 1.0 PPM MERCURY STD & 1.05 (1058 RECOVERY) \\
\hline SOLID BLANK & BLANK & $<0.004$ \\
\hline LIQUID BLANK & BLANK & $<0.004$ \\
\hline
\end{tabular}

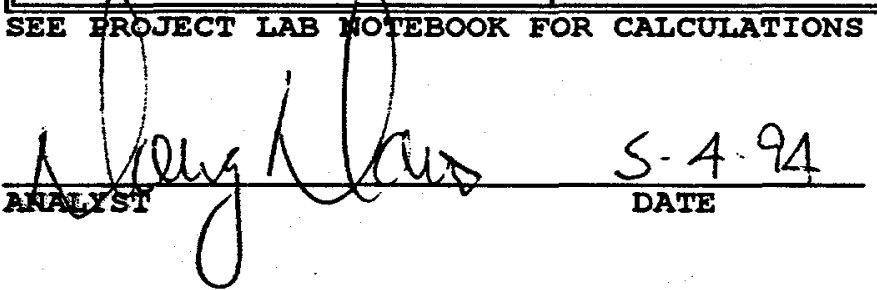




\section{ARS/ECOTEK}

\section{LEFPC SCOPING TEST RESULTS}

\begin{tabular}{|l|l|l||}
\hline \multicolumn{1}{|c|}{ SAMPLE } & \multicolumn{1}{|c|}{ SAMPLE NUMBER } & \multicolumn{1}{|c||}{ SULFUR RESULTS (PPM) } \\
\hline CALIBRATION CHECK STD & 1000 PPM SULFUR STD & 988 (98.8\% RECOVERY) \\
\hline QC CHECK STANDARD & 500 PPM SULFUR STD & 483 (96.68 RECOVERY) \\
\hline CONDENSER LIQUID & $1661 \mathrm{~B}-008$ & 9.6 \\
\hline CONDENSER LIQUID & $1661 \mathrm{~B}-018$ & 17.5 \\
\hline CONDENSER LIQUID-SPIKE & $\begin{array}{l}1661 \mathrm{~B}-018+5 \text { PPM } \\
\text { SULFUR SPIKE }\end{array}$ & $(1088$ RECOVERY) \\
\hline CONDENSER LIQUID & $1661 \mathrm{~B} 050594-S 029$ & 28.4 \\
\hline QC CHECK STANDARD & 500 PPM SULFUR STD & 479 (95.88 RECOVERY) \\
\hline CONDENSER SOLIDS & $1661 \mathrm{~B}-008 R$ & 857 \\
\hline \hline CONDENSER SOLIDS & $1661 \mathrm{~B}-018$ & 675 \\
\hline CONDENSER SOLIDS-SPIKE & $\begin{array}{l}1661 \mathrm{~B}-018+5 \text { PPM } \\
\text { SULFUR SPIKE }\end{array}$ & $(968$ RECOVERY) \\
\hline \hline CONDENSER SOLIDS & $1661 \mathrm{BO50595-SO29}$ & 686 \\
\hline QC CHEGA STANDARD & 500 PPM SULFUR STD & 489 (97.88 RECOVERY) \\
\hline
\end{tabular}

SEE PROAEOT IAB NOTHOOF FOR CALCULATIONS AND INDIVIDUAL RESULTS

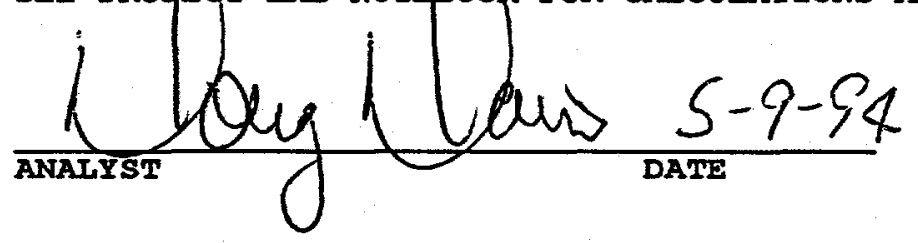




\section{ARS/ECOTEK}

\section{LEFPC SCOPING TEST RESULTS}

\begin{tabular}{|l|l|l|}
\hline \multicolumn{1}{|c|}{ SAMPLE } & \multicolumn{1}{|c|}{ SAMPLE NUMBR } & MERCURY RESULTS (PPM) \\
\hline CONDENSER RINSE & $1661 \mathrm{~B}-021$ & 0.04 \\
\hline CONDENSER RINSE + SPIKE & $\begin{array}{l}1661 \mathrm{~B}-021+0.5 \text { UG HG } \\
\text { SPIKE }\end{array}$ & $98 \%$ SPIKE RECOVERY \\
\hline CONDENSER SOLUTION & $1661 \mathrm{BO50594-S029}$ & 1.1 \\
\hline $\begin{array}{l}\text { TREATED CONDENSER } \\
\text { SOLUTION }\end{array}$ & $1661 \mathrm{~B}-030$ & 0.09 \\
\hline QC CHECK STANDARD & 1 PPM CHECK STD & 1.01 (1018 RECOVERY) \\
\hline \hline BLANK & BLANR & 0.03 \\
\hline
\end{tabular}

SEE PROJECT IAB NOFEEOK FOR CAICULATIONS AND INDIVIDUAL RESULTS

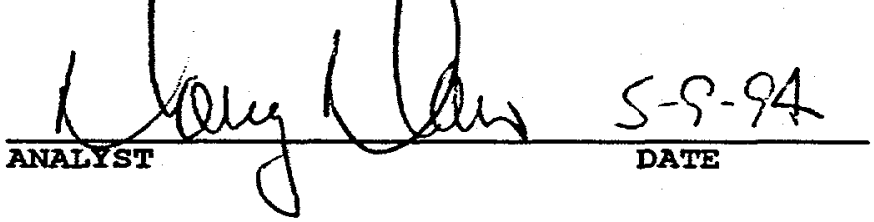




\section{ARS/ECOTEK}

\section{LEFPC SCOPING TEST RESULTS \\ (REVISED)}

\begin{tabular}{|l|l|l||}
\hline \multicolumn{1}{|c|}{ SAMPLE } & \multicolumn{1}{|c|}{ SAMPLE NUMBER } & MERCURY RESULTS (PPM) \\
\hline CONDENSER RINSE & $1661 \mathrm{~B}-021$ & 0.04 \\
\hline CONDENSER RINSE + SPIKE & $\begin{array}{l}\text { 1661B-021 }+0.5 \text { UG HG } \\
\text { SPIKE }\end{array}$ & 988 SPIKE RECOVERY \\
\hline CONDENSER LIQUID & $1661 \mathrm{B050594-S029}$ & 1.1 \\
\hline $\begin{array}{l}\text { TREATED CONDENSER } \\
\text { SOLUTION }\end{array}$ & $1661 \mathrm{~B}-030$ & 0.09 \\
\hline \hline QC CHECK STANDARD & 1 PPM CHECK STD & 1.01 (1018 RECOVERY) \\
\hline \hline BLANK & BLANX & 0.03 \\
\hline \hline CONDENSER LIQUID & $1661 \mathrm{~B}-008 R$ & 0.04 \\
\hline
\end{tabular}

SEE PRO HCT LAB NOT EPOK FOR CALCULATIONS AND INDIVIDUAL RESULTS

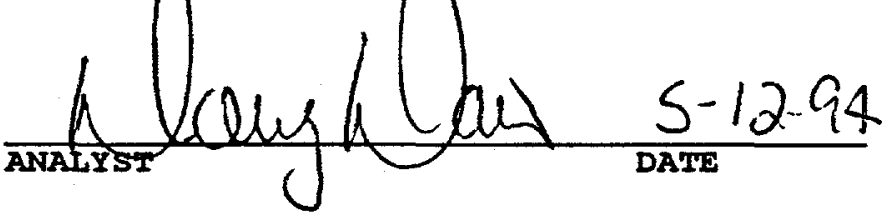




\section{ARS/ECOTEK}

\section{LEFPC SCOPING TEST RESULTS}

\begin{tabular}{|c|c|c|}
\hline SAMPLE & SAMPIE NUMBER & MGRCURY RESULTS (PIM) \\
\hline TREATED SOIL & 1661B050594-P022 & 20 \\
\hline TREATED SOIL & 1661B050594-P023 & 22 \\
\hline TREATED SOIL & 1661B050594-PO24 & 19 \\
\hline TREATED SOIL & 1661B050594-P025 & 18 \\
\hline FEED & 1661B050594-F026 & 213 \\
\hline FEED & 1661B050594-F027 & 214 \\
\hline FEED & 1661B050594-F028 & 211 \\
\hline CONDENSER SOLIDS & 1661B050594-S029 & 775 \\
\hline $\begin{array}{l}\text { SPIKE OF 1661B050594- } \\
\text { P022 }\end{array}$ & 1661B050594-P022-SPIKE & 99.28 RECOVERY \\
\hline $\begin{array}{l}\text { SPIKE OF 1661B050594- } \\
\text { F026 }\end{array}$ & 1661B050594-F026-SPIKE & 100.48 RECOVERY \\
\hline QC CHECK STANDARD \#1 & 1.0 PPM MERCURY STD & 0.94 (948 RECOVERY) \\
\hline QC CHECK STANDARD \#2 & 1.0 PPM MERCURY STD & 0.93 (938 RECOVERY) \\
\hline BLANK \#1 & BLANK & $<0.004$ \\
\hline BLAWK $\# 2$ & BLANK & $<0.004$ \\
\hline
\end{tabular}

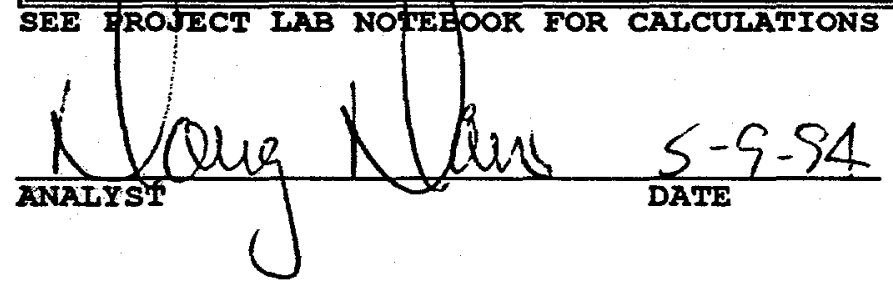




\section{ARS/ECOTEK}

\section{LEEPC SCOPING TEST RESULTS}

\begin{tabular}{|c|c|c|}
\hline SAMPLE & SAMPLE NUMBER & MERCURY RESULTS (PRM) \\
\hline QC CHECK STD & 1 PPM STD & 0.97 (978 RECOVERY) \\
\hline FEED & 1661B051094-F037 & 285 \\
\hline FEED & 1661B051094-F038 & 259 \\
\hline FEED & 1661B051094-F039 & 243 \\
\hline FEED & 1661B051094-F040 & 531 \\
\hline FEED & 1661B051094-F041 & 354 \\
\hline FEED & 1661B051094-F042 & 202 \\
\hline $\begin{array}{l}\text { SCRUBBER CLEANOUT- } \\
\text { SOLIDS }\end{array}$ & 1661B-031 & 401 \\
\hline CONDENSER SOLIDS & 1661B051094-052 & 302 \\
\hline FIELD BLANK & FIELD BLANK & $<0.004$ \\
\hline BLANK & BLANK & $<0.004$ \\
\hline
\end{tabular}

SEE PROUECT LAB NOTEBOOK FOR CALCULATIONS AND INDIVIDUAL RESULTS

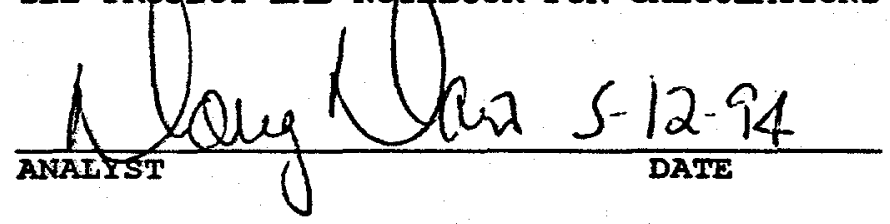




\section{ARS/ECOTEK}

\section{LEFPC SCOPING TEST RESULTS}

\begin{tabular}{|c|c|c|}
\hline SAMPLE & SAMPLE NUMBER & MERCURY RESULTS (PPM) \\
\hline QC CAECK STD & 1.0 PPM MERCURY STD & 1.02 (1028 RECOVERY) \\
\hline FEED & 1661B051094-F035 & 253 \\
\hline FEED + 0.5 PPM SPIKE & 1661B051094-F035-SPIKE & 1018 RECOVERY \\
\hline FEED & 1661B051094-F036 & 286 \\
\hline TREATED SOIL & 1661B051094-P043 & 23 \\
\hline $\begin{array}{l}\text { TREATED SOIL + } 0.5 \text { PPM } \\
\text { SPIRE }\end{array}$ & 1661B051094-P043-SPIKE & 105\% RECOVERY \\
\hline TREATED SOIL & 1661B051094-P044 & 16 \\
\hline TREATED SOIL & $1661 \mathrm{~B} 051094-\mathrm{P} 045$ & 23 \\
\hline TREATED SOIL & 1661B051094-P046 & 23 \\
\hline TREATED SOIL & 1661B051094-P047 & 21 \\
\hline TREATED SOIL & 1661B051094-P048 & 19 \\
\hline TREATED SOIL & 1661B051094-P049 & 20 \\
\hline TREATED SOIL & 1661B051094-P050 & 24 \\
\hline TREATED SOIL & 1661B051094-P051 & 15 \\
\hline BLANK & BLANK & $<0.004$ \\
\hline
\end{tabular}

SEE PROJJCT IAB NOTCBOOOK FOR CAICULATIONS AND INDIVIDUAI RESULTS

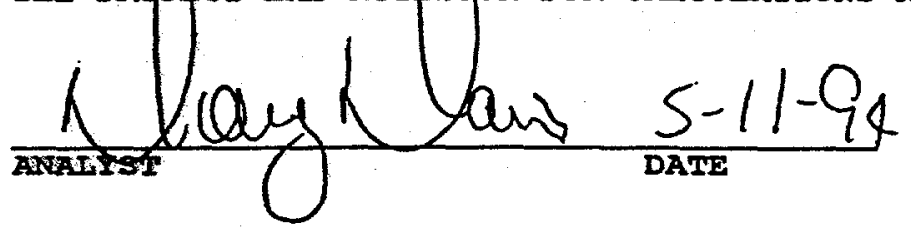




\section{ARS/ECOTEK}

\section{LEFPC SCOPING TEST RESULTS}

\begin{tabular}{|l|l|l|}
\hline \multicolumn{1}{|c|}{ SAMPLE } & \multicolumn{1}{|c|}{ SAMPLE NUMBER } & MERCURY RESULTS (PIM) \\
\hline QC CHECK STANDARD & 1 PPM MERCURY STD & 0.85 (858 RECOVERY) \\
\hline \hline TREATED SCRUBBER & $1661 \mathrm{~B}-053$ & 0.21 \\
\hline $\begin{array}{l}\text { TREATED SCRUBBER }+0.5 \\
\text { UG MERCURY SPIKE }\end{array}$ & $1661 \mathrm{~B}-053-$ SPIKE & 1128 RECOVERY \\
\hline SCRUBBER CLEANUP & $1661 \mathrm{~B}-032$ & 0.31 \\
\hline BLANK & BLANK & $<0.004$ \\
\hline SCRUBBER CLEANUP & $1661 \mathrm{~B}-031$ & 0.20 \\
\hline CONDENSER LIQUID & $1661 \mathrm{~B}-052$ & 1.50 \\
\hline RAW WATER LIQUID & $1661 \mathrm{~B}-055$ & 0.18 \\
\hline \hline TREATED WATER & $1661 \mathrm{~B}-056$ & 0.20 \\
\hline \hline BLANK & BLANK & 0.005 \\
\hline
\end{tabular}

SEE PROJECT IAA ANPTEBOOK FOR CALCULATIONS AND INDIVIDUAL RESULTS

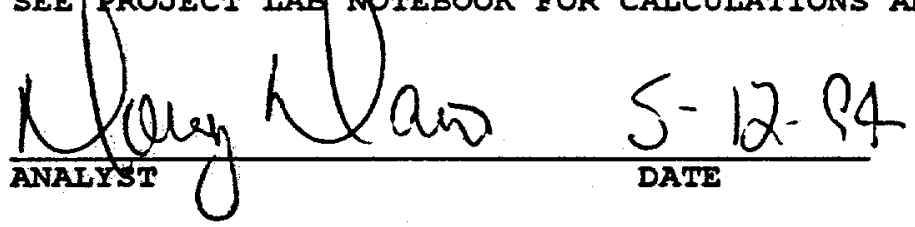




\section{ARS/ECOTER}

\section{LEFPC SCOPING TEST RESULTS}

\begin{tabular}{|l|l|l|}
\hline \multicolumn{1}{|c|}{ SAMPLE } & \multicolumn{1}{|c|}{ SAMPLE NUMBER } & MERCURY RESULTS (PPM) \\
\hline \hline QC CHECK STANDARD & 1 PPM MERCURY STD & 1.03 (1038 RECOVERY) \\
\hline \hline RAW WATER SOLIDS & $1661 \mathrm{~B}-055$ & 2363 \\
\hline \hline COLD TRAP & $1661 \mathrm{~B}-033$ & 4.02 \\
\hline \hline COLD TRAP & $1661 \mathrm{~B}-054$ & 24.7 \\
\hline \hline $\begin{array}{l}\text { COLD TRAP + 0.5 UG } \\
\text { MERCURY SPIKE }\end{array}$ & $1661 \mathrm{~B}-054-$ SPIKE & $1178 \mathrm{RECOVERY}$ \\
\hline \hline SWIPE FIELD BLANK & $1661 \mathrm{~B}-057$ & $<0.004$ \\
\hline \hline SWIPE \#1 (DESORBER) & $1661 \mathrm{~B}-058$ & $<0.004 \mathrm{ug} / \mathrm{cm}^{2}$ \\
\hline \hline $\begin{array}{l}\text { SWIPE \#1 + O.5 ug } \\
\text { MERCURY SPIKE }\end{array}$ & $1661 \mathrm{~B}-058-S P I K E$ & $1108 \mathrm{RECOVERY}$ \\
\hline \hline $\begin{array}{l}\text { SWIPE \#2 (CROSSOVER } \\
\text { PIPE) }\end{array}$ & $1661 \mathrm{~B}-059$ & $0.04 \mathrm{ug} / \mathrm{cm}^{2}$ \\
\hline \hline \begin{tabular}{l} 
SWIBE \#3 (CONDENSER) \\
\hline \begin{tabular}{l} 
BLANK \\
\hline
\end{tabular}
\end{tabular} & $1661 \mathrm{~B}-060$ & $0.14 \mathrm{ug} / \mathrm{cm}^{2}$ \\
\hline
\end{tabular}

SEE PROJECT LAB NOTHBOQK FOR CALCULATIONS AND INDIVIDUAL RESULTS

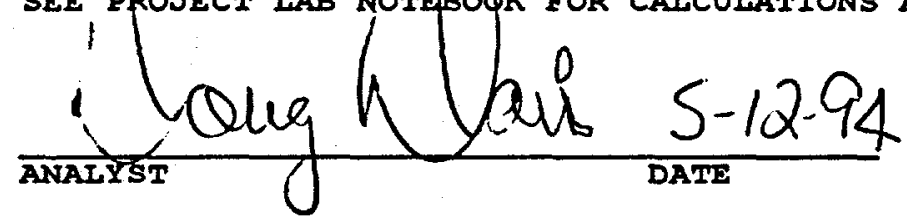


50

PROJECT $\ldots . .$.

LETTER OF AUTHORIZATION NO.: 1661B-001

February 2, 1994

Page 12 of 16

ATTACHMENT 3

LEFPC SAMPLE COLLECTION LOG

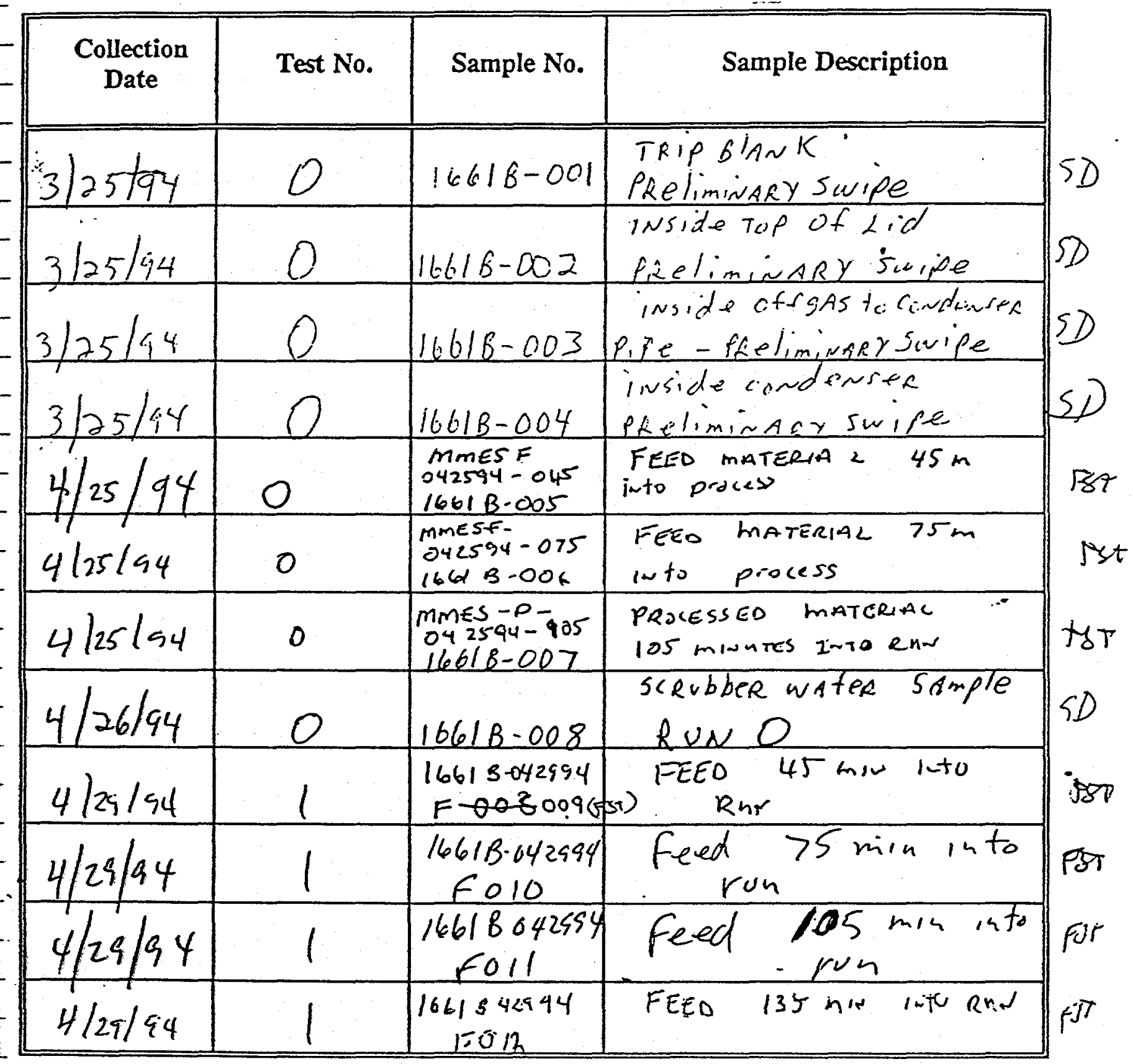


70T-94-0001 SY'S-50-02

LETTER OF AUTHORIZATION NO.: 1661B-001

February 2, 1994

Page 12 of 16

ATTACHMENT 3

LEFPC SAMPLE COLLECTION LOG

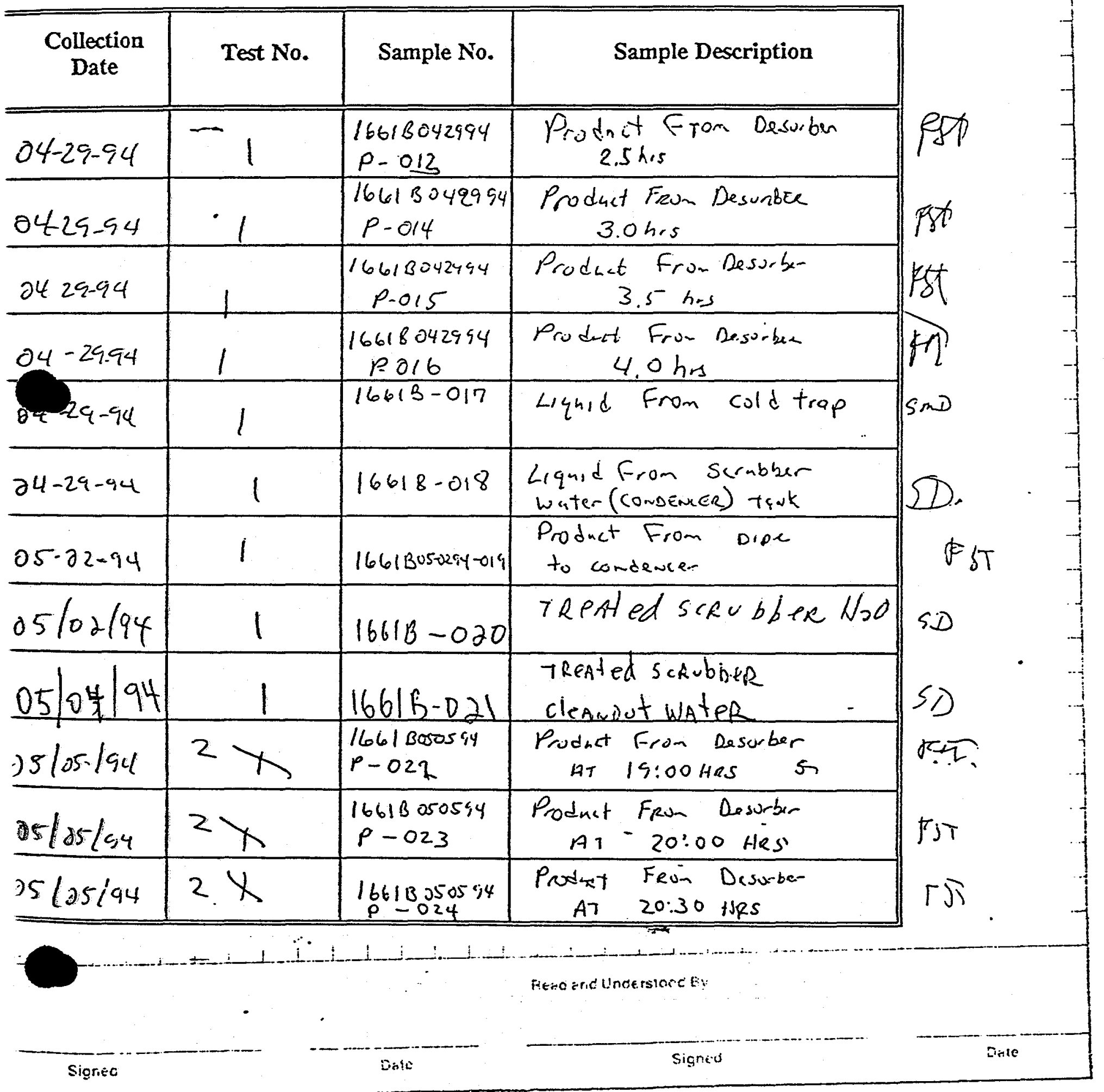


52

PROJECT.

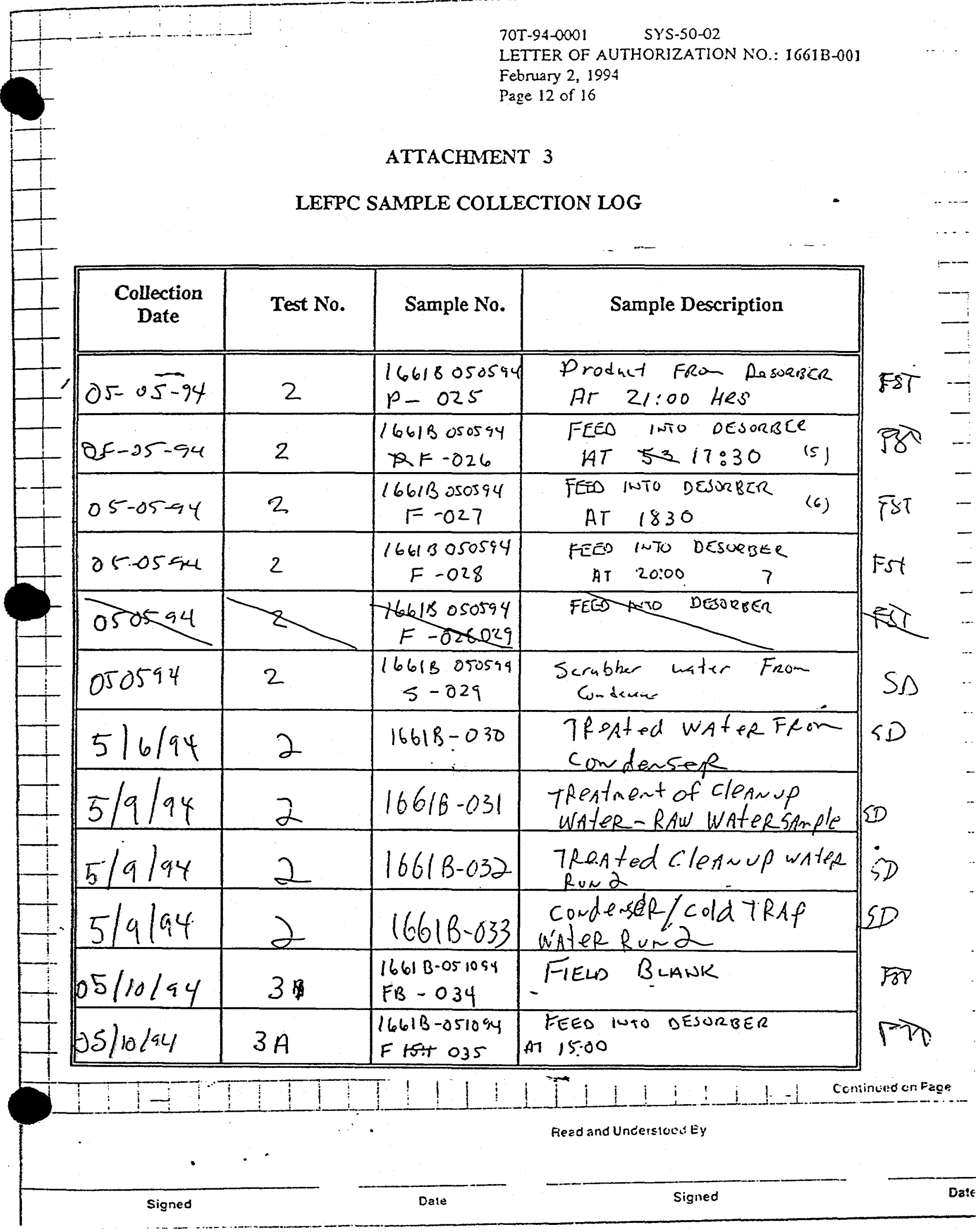


70T-94-0001

SYS-50-02

LETTER OF AUTHORIZATION NO.: 1661B-001

February 2, 1994

Page 12 of 16

\section{ATTACHIMENT 3}

\section{LEFPC SAMPLE COLLECTION LOG}

\begin{tabular}{|c|c|c|c|c|}
\hline $\begin{array}{l}\text { Collection } \\
\text { Date }\end{array}$ & Test No. & Sample No. & Sample Description & \\
\hline $05 / 0-54$ & $3 A$ & $\begin{array}{l}1661 B 051094 \\
F-036\end{array}$ & $\begin{array}{l}\text { FEED IHTO OESORBER } \\
\text { AT } 16: 00\end{array}$ & pis \\
\hline $0510-94$ & $3 a$ & $\begin{array}{c}16613051054 \\
F 037 \\
\end{array}$ & $\begin{aligned} & \text { FEED } 1 w+0 \text { DESUEBER } \\
& \text { AT } 16: 30\end{aligned}$ & $5 \pi$ \\
\hline 851094 & $3 B$ & $\begin{array}{l}1661 B 051094 \\
-F-038\end{array}$ & $\begin{array}{l}\text { FEED IWTO DESORBER } \\
\text { BT } 17: 30\end{array}$ & FST. \\
\hline 052094 & $3 B$ & $\begin{array}{l}16613051894 \\
-F \cdot 039 \\
\end{array}$ & $\begin{array}{l}\text { FEED IWTO DESURBE? } \\
\text { AT 18:00 }\end{array}$ & Ps \\
\hline 051094 & $3 B$ & $\begin{array}{l}16613 \text { o51054 } \\
F \text { o3 } 00510\end{array}$ & $\begin{array}{l}\text { FEED ITTO DEsuesce } \\
\text { AT 18:30 }\end{array}$ & par \\
\hline 051094 & $3 B$ & $\begin{array}{l}16618051094 \\
F-041\end{array}$ & $\begin{array}{l}\text { FEED inro DESOROER } \\
\text { AT } 19: 00\end{array}$ & $\pi$ \\
\hline 257094 & $3 B$ & $\begin{array}{l}1661 B 251094 \\
F-042\end{array}$ & $\begin{array}{c}\text { FEED IWTO DESORDER } \\
\text { nt } 19: 30\end{array}$ & 381 \\
\hline 051094 & $3 A$ & $\begin{array}{l}1661 B 051094 \\
P-043\end{array}$ & $\begin{array}{c}\text { PrODMAT FROM DESORBER } \\
\text { AT } 16: 00\end{array}$ & 75 \\
\hline 051094 & 34 & $\begin{array}{c}1661 B 051094 \\
P-244\end{array}$ & $\begin{array}{c}\text { Produt Fed Desorber } \\
\text { At 16:30. }\end{array}$ & pso \\
\hline 051094 & $3 n$ & $\begin{array}{c}1661 B 251094 \\
P-245\end{array}$ & $\begin{array}{c}\text { Prodad Fou Desurber } \\
\text { AT 17:00 }\end{array}$ & ps \\
\hline 051094 & $3 B$ & $\begin{array}{c}16613051044 \\
P-046 \\
\end{array}$ & $\begin{array}{l}\text { Product Frow Desorber } \\
-\quad \text { At } 18: 00\end{array}$ & FST \\
\hline 251094 & $3 B$ & $\begin{array}{l}1661 \text { B 051094 } \\
-P-047 \\
\end{array}$ & $\begin{array}{l}\text { Prodint From Dosorber } \\
A_{1} \quad 18: 30\end{array}$ & 387 \\
\hline \multicolumn{5}{|c|}{ Fead and Understood By } \\
\hline & & & Signed & Date \\
\hline
\end{tabular}


PROJECT

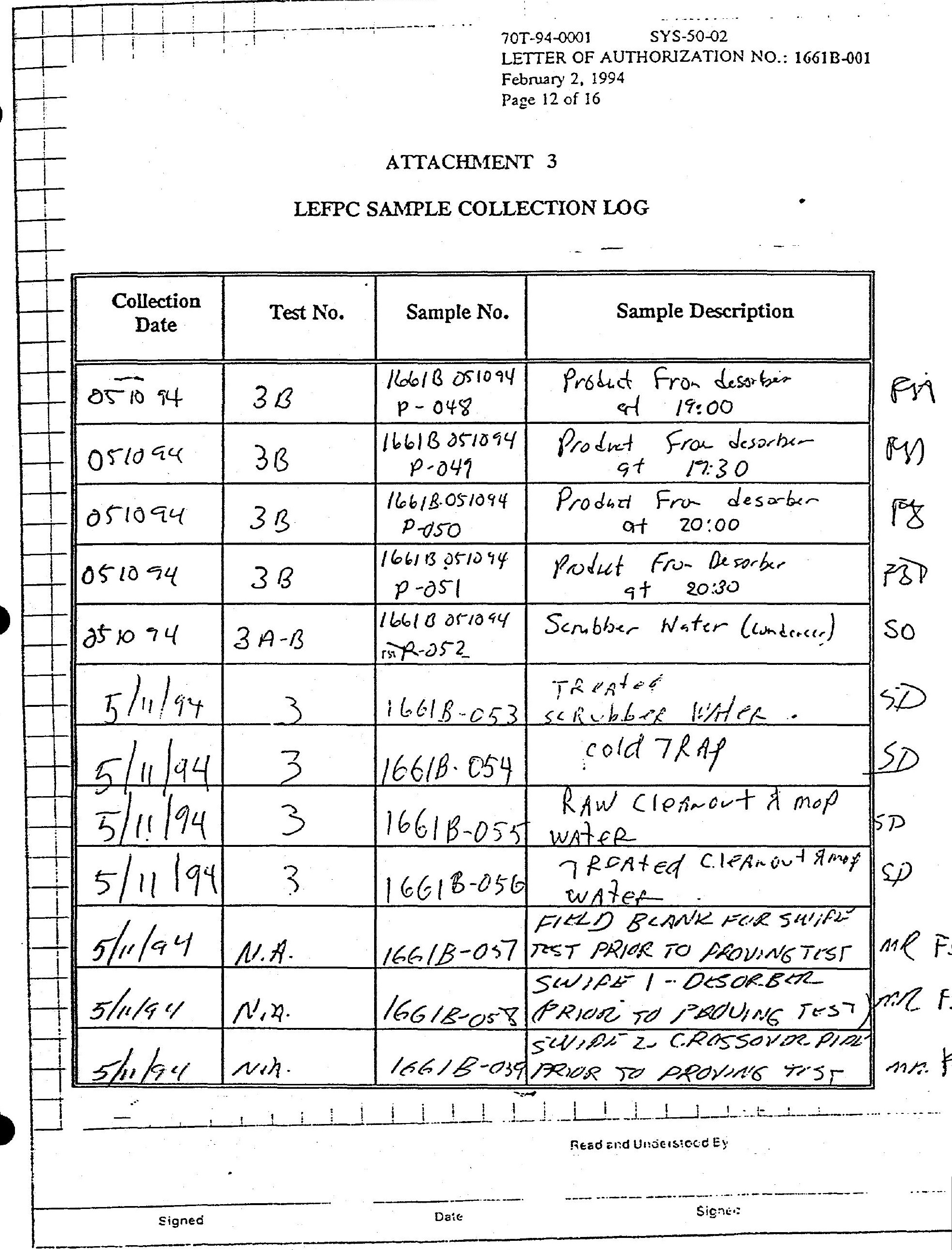

